\title{
Ethologische und morphologische Untersuchung von Hybriden der Feldheuschreckenarten
} Stenobothrus clavatus und Stenobothrus rubicundus

\author{
Dissertation \\ zur Erlangung des mathematisch-naturwissenschaftlichen Doktorgrades \\ "Doctor rerum naturalium" \\ der Georg August Universität Göttingen
}

vorgelegt von

Jan Eberhard Sradnick

aus Cottbus

Göttingen 2010 
Mitglieder des Betreuungsausschusses:

Referent: Prof. Dr. Norbert Elsner

Koreferent: Prof. Dr. Andreas Stumpner

Tag der mündlichen Prüfung: 


\section{Inhaltsverzeichnis}

1. Einleitung ....................................................................................................................1

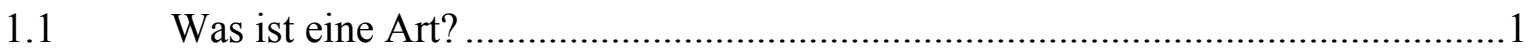

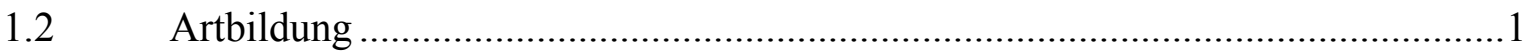

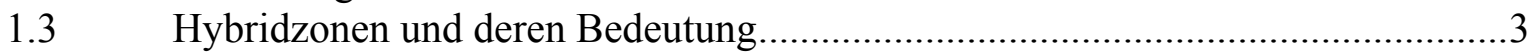

1.4 Orthopteren als Untersuchungsobjekt für Artbildungsprozesse ..........................5

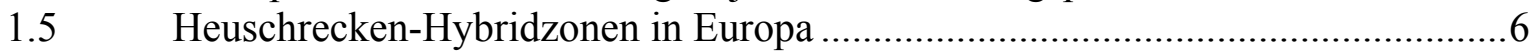

1.6 Inselpopulationen und deren Bedeutung bei der Artbildung .............................

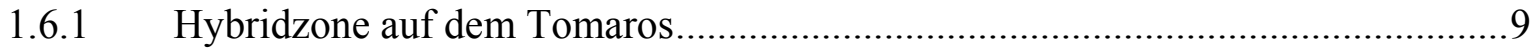

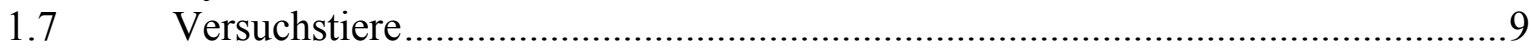

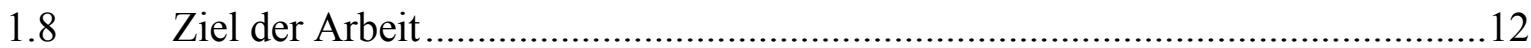

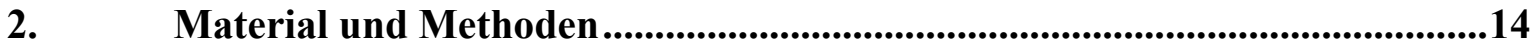

2.1 Vorkommen der zum Vergleich herangezogenen Populationen von St.

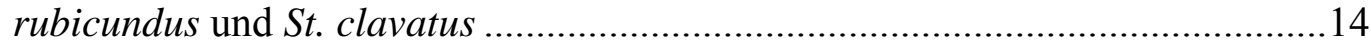

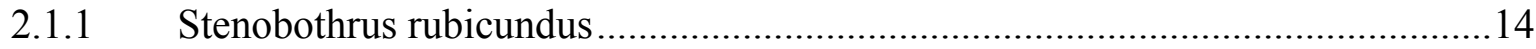

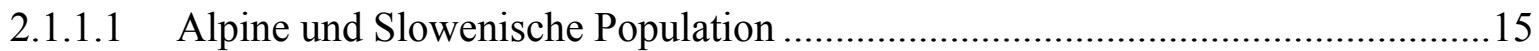

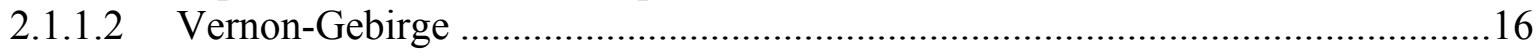

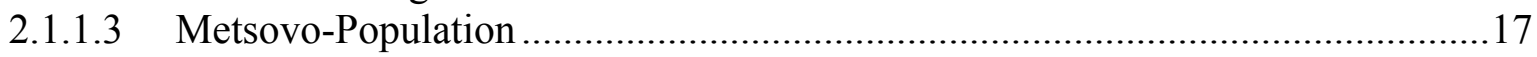

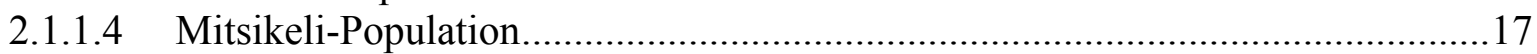

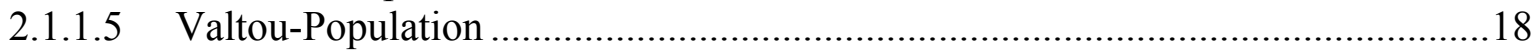

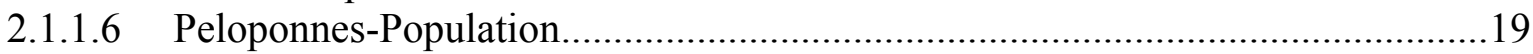

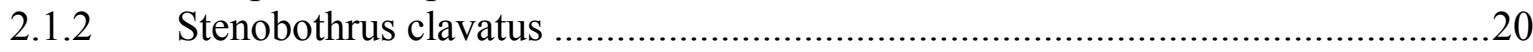

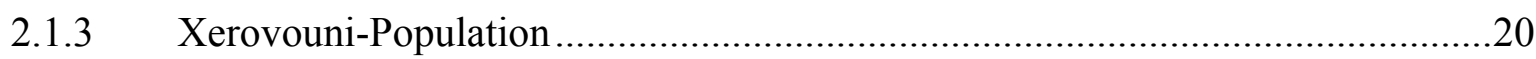

2.2 Tomaros-Populationen von St. rubicundus, St. clavatus und deren Hybriden ....21

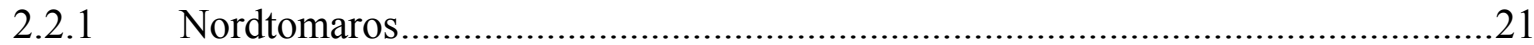

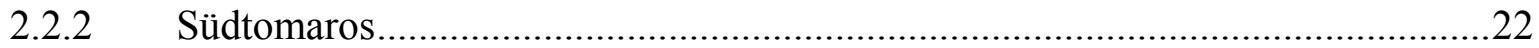

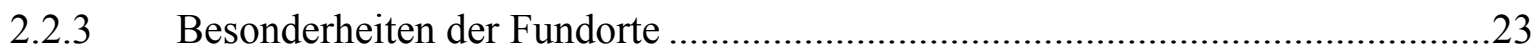

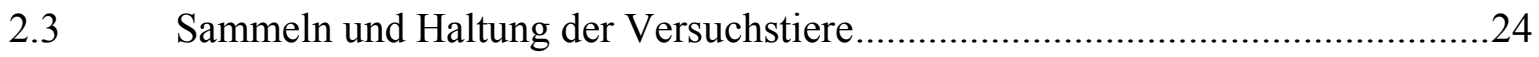

2.4 Morphometrische Vermessung der Vorderflügel..........................................24

2.4.1 Aufnahme der Flügel und Setzen von Messpunkten..........................................24

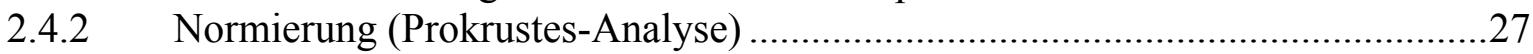

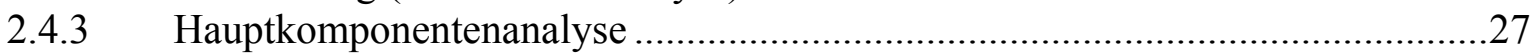

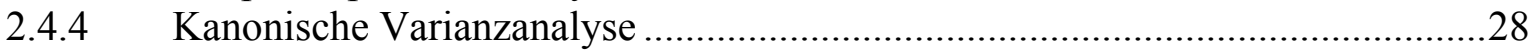

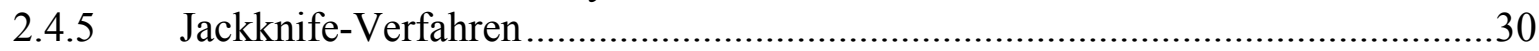

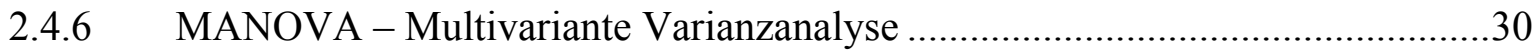

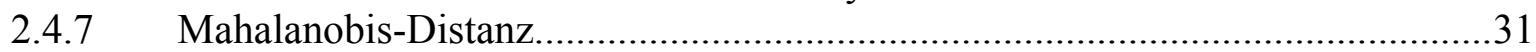

2.4.8 Mahalanobis-Distanz-Differenz, Merkmalsanteile und Hybridindex....................32

2.4.9 Bestimmung des Flächeninhalts des Vorderflügels und der Länge des Femurs -

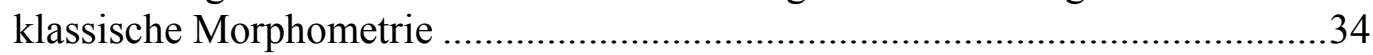

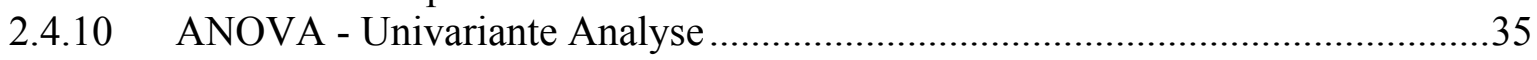

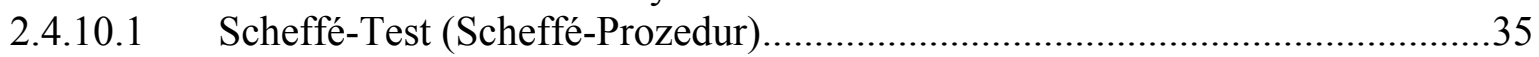

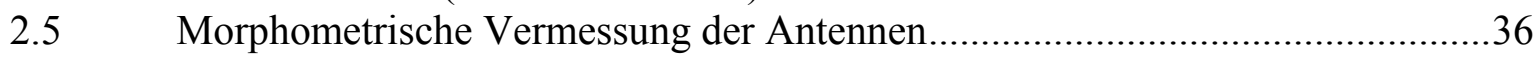

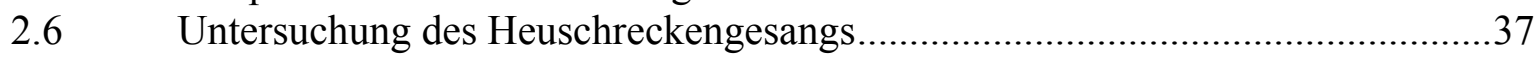

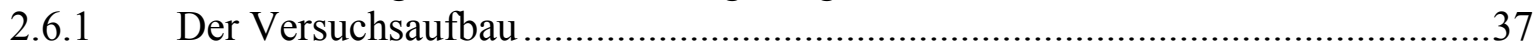

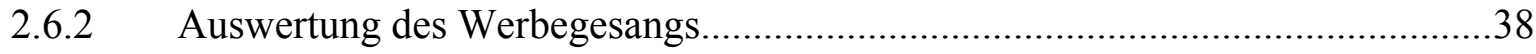

2.6.3 Hybridindices und Merkmalsanteile der Gesangsmerkmale................................39 


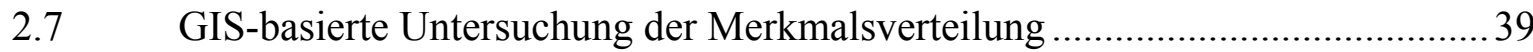

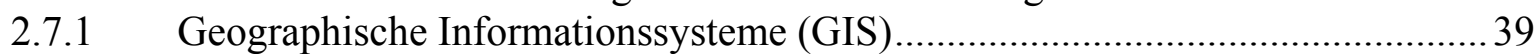

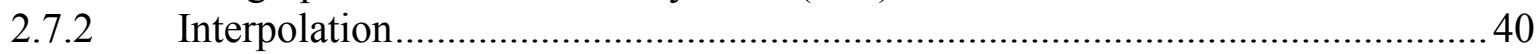

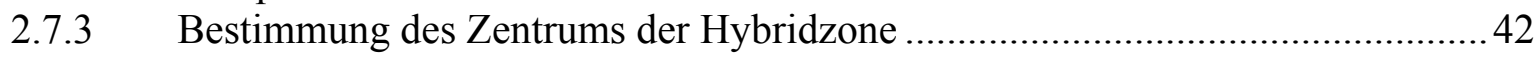

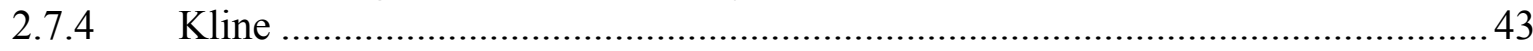

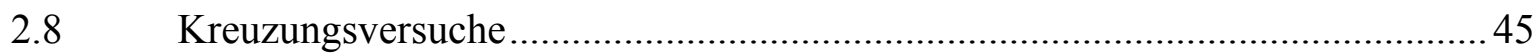

2.9 Betrachtung von Klimadaten zur Untersuchung der ökologischen Nische ......... 45

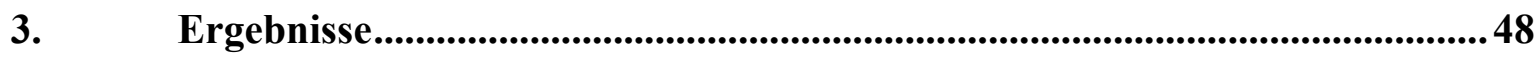

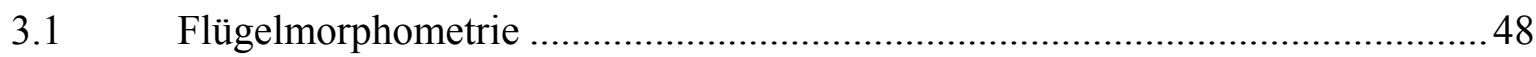

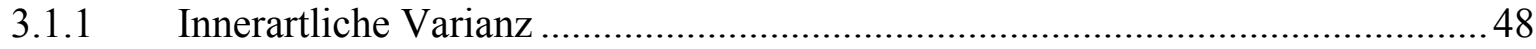

3.1.1.1 St. rubicundus der griechischen und slowenischen Populationen ....................... 48

3.1.1.2 Vergleich zwischen den griechischen St. rubicundus Populationen .................... 52

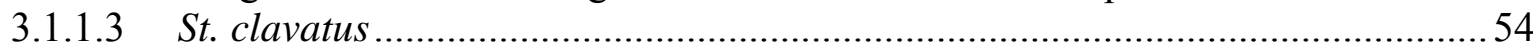

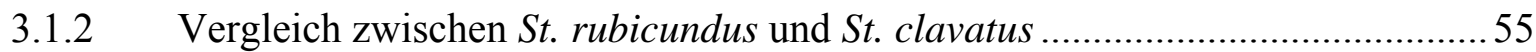

3.1.3 Einordnung der zwischenartlichen Varianz mit Hilfe von Außengruppen. .........58

3.1.4 Populationen des Tomarosgebirges -klassische Morphometrie- .............................61

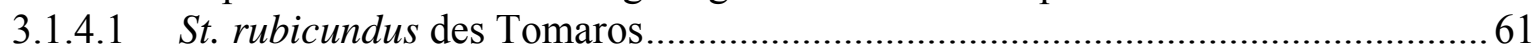

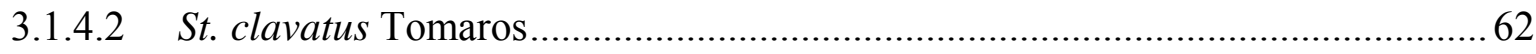

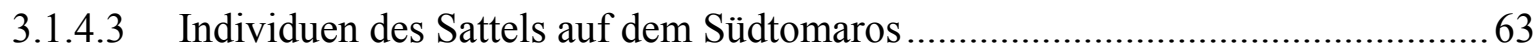

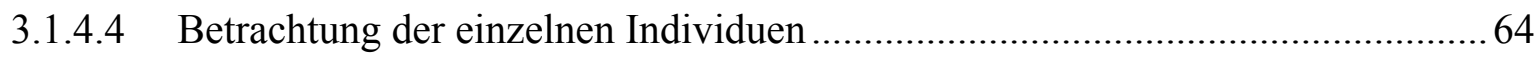

3.1.5 Geometrische Morphometrie an einzelnen Individuen des Tomaros ...................66

3.1.5.1 Einordnung der Individuen des Tomaros beider Referenzgruppen .......................67

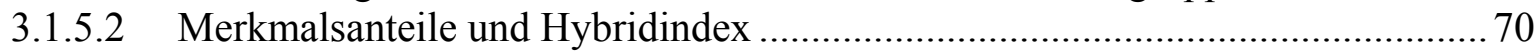

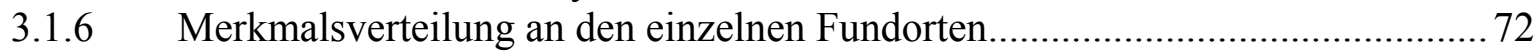

3.1.6.1 Griechische St. rubicundus Populationen außerhalb des Tomaros-Gebirges ...... 72

3.1.6.2 Charakterisierung der Tomaros-Populationen von St. clavatus, St. rubicundus und deren Hybriden aufgrund der Vorderflügelmorphologie............................ 73

3.1.6.3 Merkmalsverteilung auf dem Tomaros-Nordmassiv (Gebiet I) .......................... 75

3.1.6.4 Merkmalsverteilung auf dem Nord- und Südhang des Tomaros-Südmassiv ...... 78

3.1.6.5 Merkmalsverteilung auf dem Osthang des Tomaros-Südmassiv ........................ 83

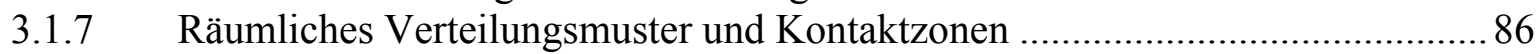

3.1.7.1 Untersuchung des räumlichen Musters der Merkmalsverteilung auf dem Tomaros

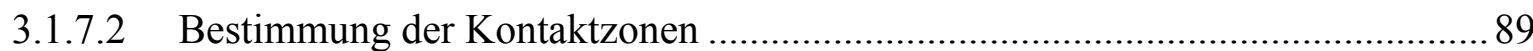

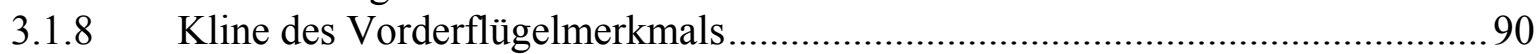

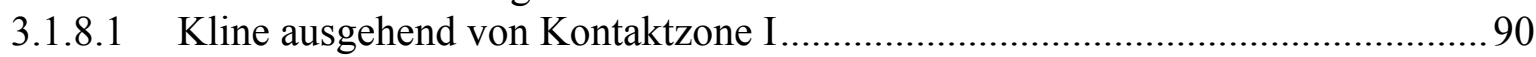

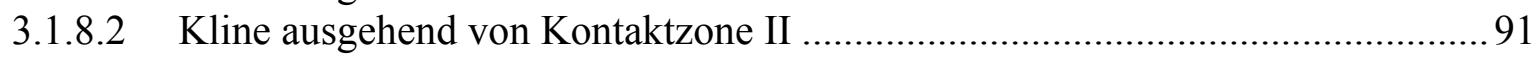

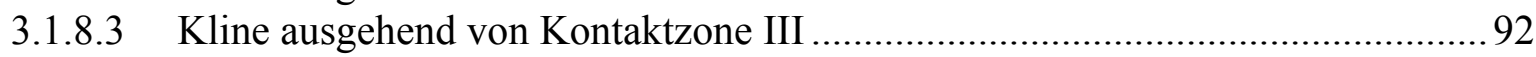

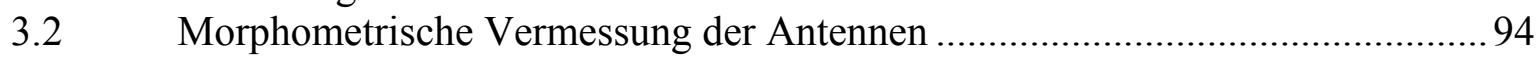

3.2.1 Zwischenartlicher Vergleich von St. clavatus und St. rubicundus ...................... 94

3.2.2 Antennenmorphologie der Individuen des Tomarosgebirges .............................. 96

3.2.3 Charakterisierung der Tomaros-Populationen von St. clavatus, St. rubicundus und deren Hybriden aufgrund der Antennenmorphologie.................................. 98

3.2.4 Merkmalsverteilung auf dem Tomaros-Nordmassiv ........................................... 99

3.2.5 Merkmalsverteilung auf dem Tomaros-Südmassiv .......................................... 101

3.2.6 Merkmalsverteilung auf dem Osthang des Südtomaros .................................... 106

3.2.7 Bestimmung der Merkmalskline auf Grundlage der Antennenmorphologie .... 109

3.2.8 Interpolierte Merkmalsverteilung des Antennenmerkmals auf dem Tomaros .. 109

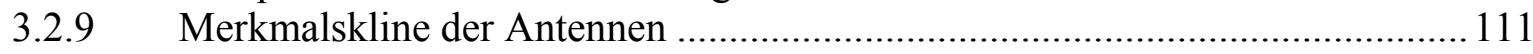


3.3 Vergleich zwischen Flügel- und Antennenmorphologie.................................. 114

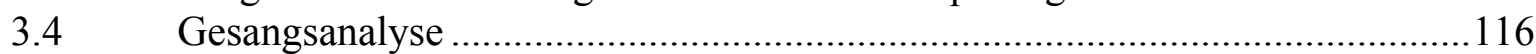

3.4.1 Werbeverhalten der Männchen von Stenobothrus rubicundus ..........................116

3.4.2 Das Werbeverhalten der Männchen von Stenobothrus clavatus.........................117

3.4.3 Werbeverhalten der Tomaros-Heuschrecken................................................... 118

3.4.4 Beschreibung der Gesangsmerkmale ................................................................119

3.4.4.1 Merkmal 1 - Lautstärkenverhältnis der Auf- und Abwärtsbewegungen in Phase I.

3.4.4.2 Merkmal 2 - Pulsdauer während der Abwärtsbewegung in Phase I ..................121

3.4.4.3 Merkmal 3 - Anzahl der Flügelschläge in Phase II..........................................122

3.4.4.4 Merkmal 4 - Verhältnis von Bein/Flügel -Pulsamplitude ...................................124

3.4.4.5 Merkmal 5 - Einfache Lautpulse im Verhältnis zu mehrfachpulsierten Lauten in

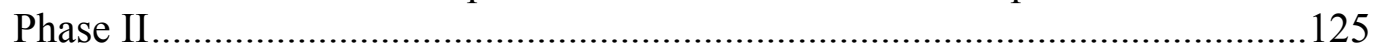

3.4.4.6 Merkmal 6 - Dauer des anhaltenden Flügelschlags am Ende der Balz...............127

3.4.4.7 Merkmal 7 - Anzahl der komplexen, hochamplitudigen Beinbewegungen.......128

3.4.4.8 Merkmal 8 - Anzahl der Beinbewegungen mit stufiger Abwärtsbewegung......129

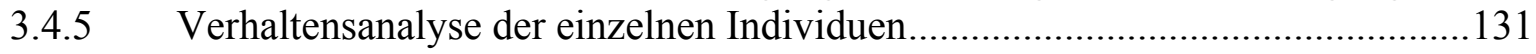

3.4.6 Verteilung des Gesangsmerkmals auf dem Tomaros........................................132

3.4.7 Interpolierte Verteilung der Gesangsmerkmale auf dem Tomaros ....................136

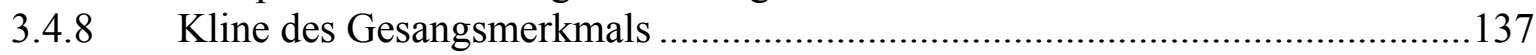

3.5 Vergleich der verschiedenen Merkmalskline ......................................................139

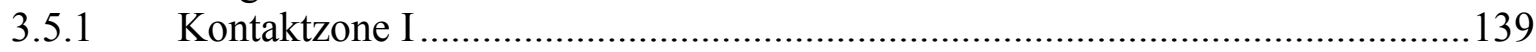

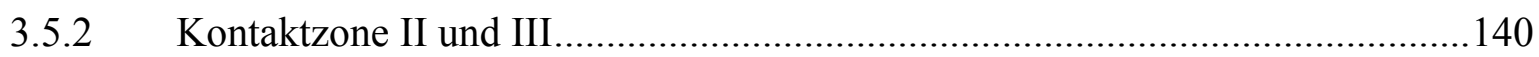

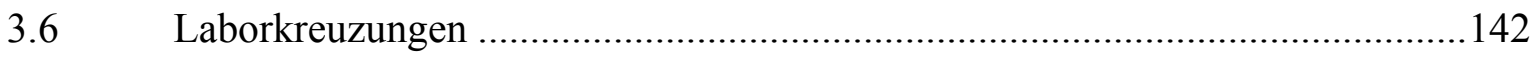

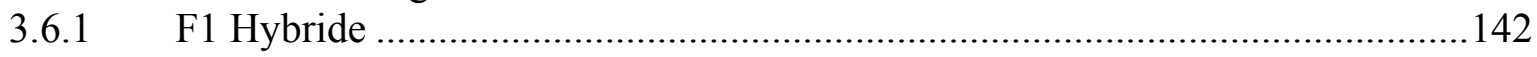

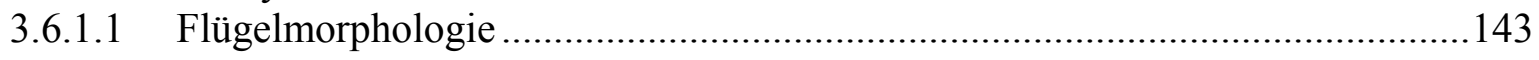

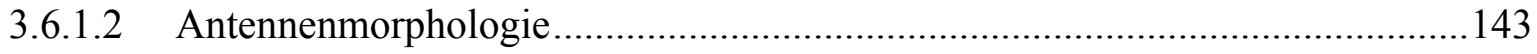

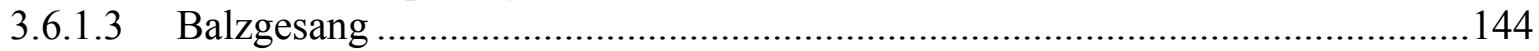

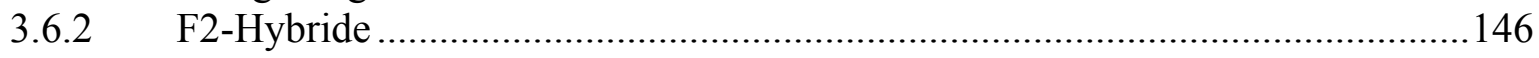

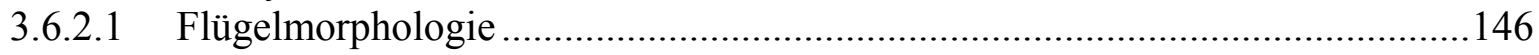

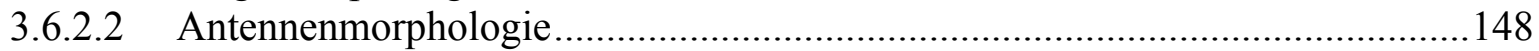

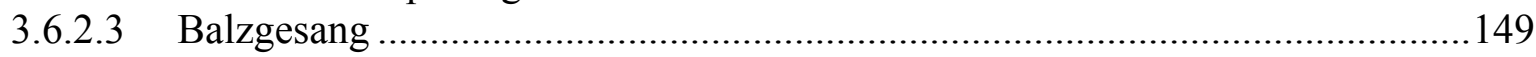

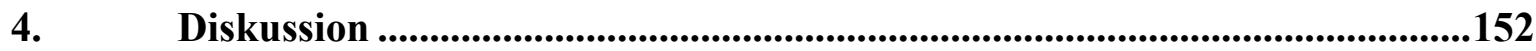

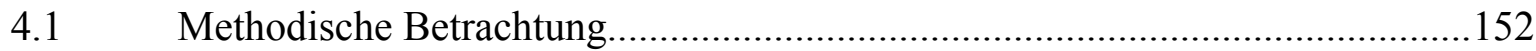

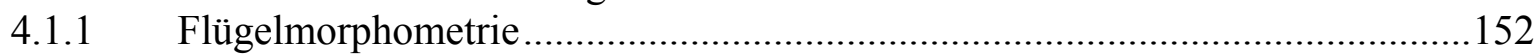

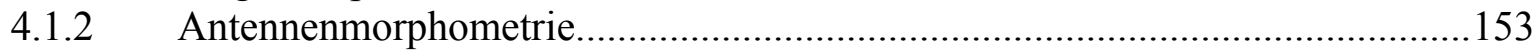

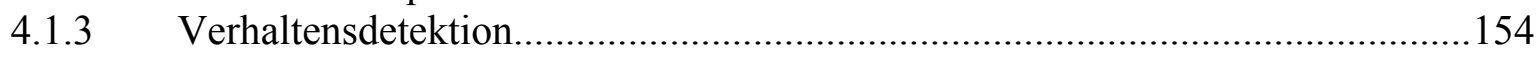

4.1.4 Untersuchungen mit Hilfe von geographischen Informationssystemen ............154

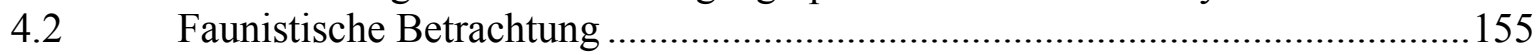

4.2.1 Populationsstruktur der Arten St. rubicundus und St. clavatus..........................155

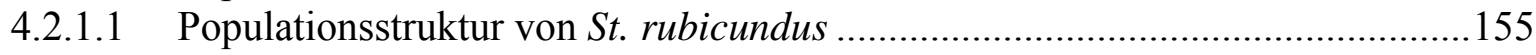

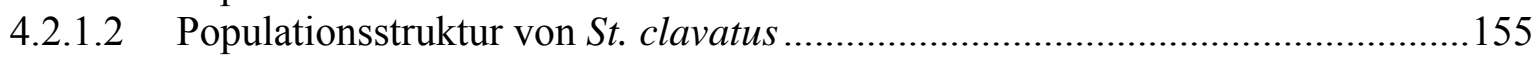

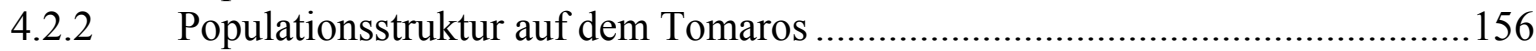

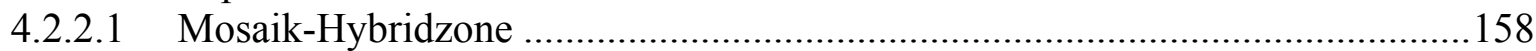

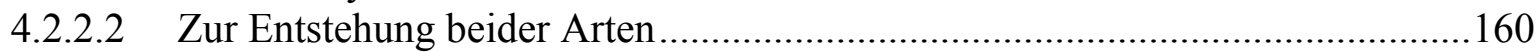

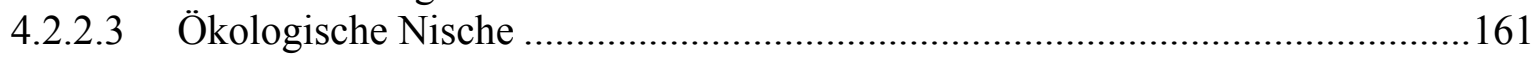

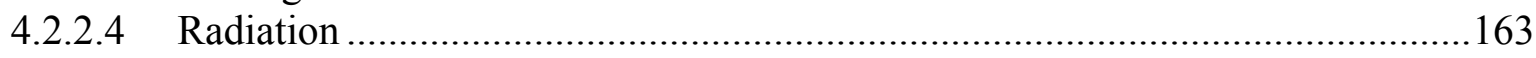

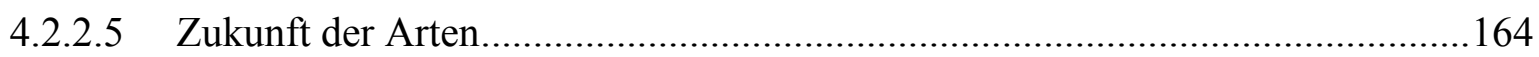

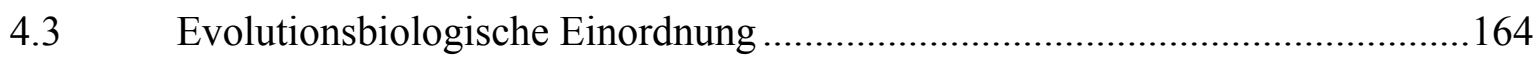




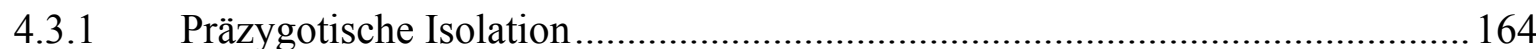

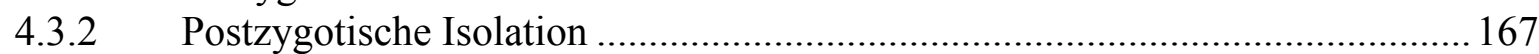

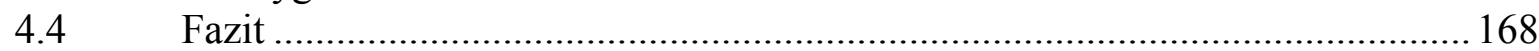

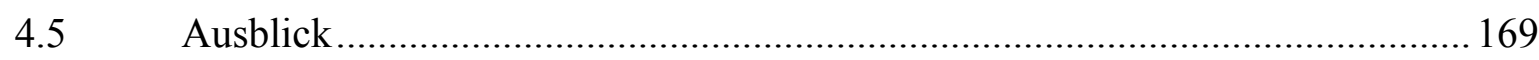

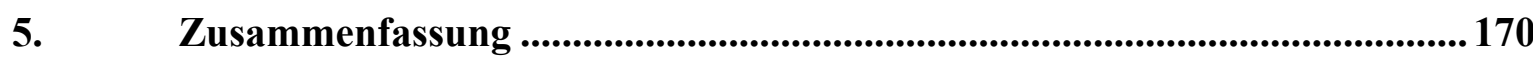

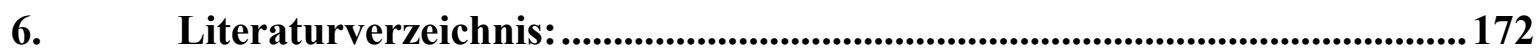

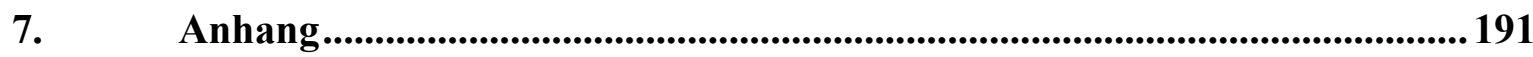

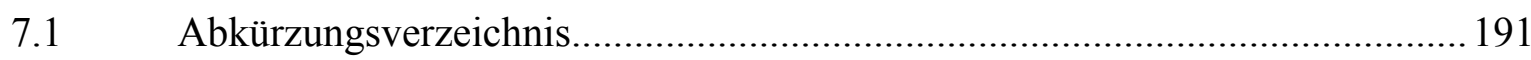




\section{Einleitung}

\subsection{Was ist eine Art?}

Charles Darwin (1859) beschrieb in seinem Buch „The origin of species“ Arten nicht als unveränderliche Einheiten, die einmalig und endgültig erschaffen sind. Vielmehr erkannte er, dass Arten sich verändern, aufspalten und so neue Arten entstehen können. Die genaueren Mechanismen dieser Artbildung sind seit 150 Jahren Gegenstand intensiver Forschungen. Dabei stellt sich immer wieder die Frage, wie Arten überhaupt zu definieren sind; vergl. hierzu Willmann (1985).

Ernst Mayr (1942) definierte Arten als: „Gruppen von sich untereinander tatsächlich oder potentiell fortpflanzender Populationen, die reproduktiv von anderen solchen Gruppen isoliert sind“. Diese Artdefinition ist in der Evolutionsbiologie vorherrschend, aber es existieren weitere Artkonzepte die mit dem Mayrschen Artkonzept konkurrieren. Arten werden unter anderem als genotypische Cluster (Mallet 1995), als phylogenetische Einheiten (de Queiroz und Donoghue 1988, Cracraft 1989, Shaw 1998) oder als evolutionäre Einheiten (Wiley 1978) bezeichnet.

In der vorliegenden Arbeit wird für die verwendeten Arten die phylogenetische Artdefinition angewandt. Eine phylogenetische Art ist eine Gruppe von Individuen, die ein bestimmtes einzigartiges Merkmal teilen, das in keiner anderen Gruppe vorkommt. Dieses sollte nicht an ein bestimmtes Geschlecht oder eine Altersgruppe der Individuen gebunden sein. Dadurch werden vor allem allopatrische Populationen, die sich gegebenenfalls zwar nur gering, aber doch in einem Merkmal unterscheiden, als Arten bezeichnet.

\subsection{Artbildung}

Bei Artbildungsprozessen (Speziation) sind vor allem die Prozesse und Mechanismen interessant, die zur Trennung der Arten, also zu ihrer reproduktiven Isolierung führen und dadurch auch eigenständig evolvieren. Die reproduktive Isolation ist also eine Bedingung für 
die Entstehung neuer Arten. Vollkommene Isolation ist bei der Entstehung neuer Arten nicht wesentlich, es ist viel wichtiger, dass eine Verbreitung der Allele zwischen den neu entstehenden Arten verhindert wird, die die Unterschiede zwischen ihnen wieder verwischen könnte (Zrzavý 2009).

Verschiedene Aspekte von Artbildungsprozessen wurden in den letzten Jahren näher untersucht. Es wurden vor allem evolutionäre Prozesse beschrieben werden, die zur Aufrechterhaltung oder Zusammenbruch von Hybridzonen beitragen. (Otte und Endler 1989, Howard und Berlocher 1998, Barton 2001, Coyne und Orr 2004). Aber die Prozesse, die der Differenzierung von Populationen zugrunde liegen, blieben weitgehend ungeklärt oder werden kontrovers diskutiert. Vor allem die Bedeutung von genetische Drift im Vergleich zur natürlichen Selektion gehört zu den offenen Fragen (Gavrilets und Hastings 1996). Ebenfalls stellt sich die Frage, ob reproduktive Selektion nur ein Nebenprodukt der verschiedenen Selektionsprozesse ist oder ob es direkte Selektion gegen Genfluss gibt (Schluter 2001). Schließlich fragt sich, ob direkte Selektion gegen Genfluss der entscheidende Mechanismus im gesamten Artbildungsprozess (Dieckmann und Doebeli 1999) ist oder ob diese nur in der finalen Phase wirkt (Noor 1999).

Ernst Mayr (1962) teilte die Speziation in zwei Grundtypen ein. Zum einen die allopatrische und zum anderen die sympatrische Speziation. Allopatrisch bedeutet, dass neue Arten in getrennten Regionen entstehen (von griechisch allos: fremd und lat. patria Vaterland). Dies sind geografisch getrennte Populationen, die sich durch genetische Drift oder Selektionsprozesse differenzieren und sich im Laufe der Zeit reproduktiv voneinander isolieren (Mayr 1963). Während der sympatrischen Artbildung (griechisch sym-: gemeinsam) bilden sich Isolationsmechanismen zwischen Individuen sich kreuzenden Populationen aus, die nicht geographisch voneinander getrennt sind (Futuyma und Mayer 1980). Die Populationen müssen sich bei der sympatrischen Artbildung trotz möglichem Genfluss voneinander abgrenzen. Weit verbreitete Modelle zur sympatrischen Artbildung beinhalten oft disruptive Selektion, also die Selektion gegen intermediäre Phänotypen. Dadurch können Populationen trotz Genfluss divergieren (Kawecki 1996, 1997, Dieckmann und Doebeli 1999, Kondrashov und Kondrashov 1999, Fry 2003). Dies kann schließlich zur Stärkung präzygotischer Isolation und so zur direkten Selektion und schließlich zu reproduktiver Isolation führen (Via 2001, Dres und Mallet 2002). Bei reproduktiver Isolation wird der Genfluss 
zwischen Populationen durch Bildung von Fortpflanzungsbarrieren (Isolationsmechanismen) unterbrochen.

In der Evolutionsbiologie unterscheidet man präzygotische (prägame) und postzygotische (postgame) Fortpflanzungsbarrieren, je nachdem, ob sie vor oder nach der Befruchtung wirken. Präzygotische Evolutionsbarrieren zeigen sich zum Bespiel in der Ablehnung von Individuen einer anderen Art als Fortpflanzungspartner. Diese Ablehnung kann in unterschiedliche Präferenzen im Werbeverhalten begründet sein (Verhaltensisolation). Aber auch unterschiedliche Zeiten oder Orte der Paarung können eine interspezifische Kopulation mit anschließender Befruchtung verhindern.

Mechanismen der postzygotischen Reproduktionsbarrieren verhindern eine normale Entwicklung der durch zwischenartliche Kreuzung entstandenen Zygote oder Embryonen. Aber auch eine herabgesetzte Lebens- oder Fortpflanzungsfähigkeit von Hybriden führt zur reproduktiven Isolation.

Präzygotische Reproduktionsbarrieren entstehen vor allem durch sexuelle Selektion, postzygotische Reproduktionsbarrieren durch die allmähliche Anreicherung von zufälligen nicht zusammenpassenden Veränderungen im Genpool zweier Arten. Somit kann eine präzygotische Isolation sehr viel schneller entstehen als eine postzygotische Barriere (Coyne, Orr 1989b, 1997). Trotz der verschiedenen Isolationsmechanismen kann es auch in der Natur zur Hybridisierung zwischen zwei; noch nicht vollständig voneinander getrennten Arten kommen (Bridle et al., 2001; Bridle, Butlin, 2002; Bailey et al., 2004; Vedenina et al., 2007). Oft führt dies zur Bildung von Hybridzonen.

\subsection{Hybridzonen und deren Bedeutung}

Als Hybridzone bezeichnet man ein Gebiet, in dem sich die Verbreitungsgebiete von zwei Arten bzw. Unterarten berühren und diese sich dort untereinander kreuzen (Schwenk 2008). Hybridzonen werden auch als , natürliche Laboratorien der Evolution“ (Hewitt 1988) oder „Fenster in die Evolutionsbiologie“ (Harrison 1990) bezeichnet. Man findet dort Individuen, die viele Generationen der zwischenartlichen Rekombination darstellen. 
Eine Hybridzone bietet so die Möglichkeit, Gene, Genkombinationen und Eigenschaften, die für die Trennung der Arten entscheidend sind, zu erforschen (Barton und Hewitt 1985, 1989; Harrison 1986). So ist es möglich, den Ausprägungszustand einer allopatrischen Artbildung durch die Betrachtung von Genfluss, Paarungsverhalten und Reproduktionserfolg, relative Fitness, Drift und Selektion zu analysieren.

Hybridzonen können Kontaktzonen von zwei neu entstehenden Arten - man spricht dann von einer primären Hybridzone - sein, können aber auch durch das Aufeinandertreffen von zwei nah verwandten Arten entstehen, die sich durch den Wegfall z.B. von ökologischen oder geographischen Barrieren erneut zusammentreffen: sekundäre Hybridzone (Barton und Hewitt 1985).

Bevor es zur Entstehung von sekundären Hybridzonen kommt, müssen sich Gruppen von Lebewesen für eine bestimmte Zeit trennen und sich separat voneinander entwickeln. Im Europa des Pleistozän (vor 2,6 Mio - 12000 Jahren) kam es oft zu kleinen und größeren Eiszeiten. Die letzte dieser Eiszeiten, das Weichsel-Glazial, hatte seinen Höhepunkt vor 18.000-20.000 Jahren. Während dieser Kältezeit lag ein großer Teil Europas unter Eismassen, die sich bis Mitteleuropa erstreckten (Abb. 1). Auch die Alpen waren permanent vergletschert, und zwischen den Alpen und den von Norden kommenden Eismassen befand sich eine artenarme Tundra (Lang 1994). Einige Arten überlebten aber in Refugien des Balkans und des nördlichen Mittelmeerraums (Cooper et al. 1995; Hewitt 1999) und bildeten in ihren Refugien allmählich unterschiedliche Merkmale aus. Vor 12.000 Jahren erwärmte sich das Klima, und viele Arten drängten wieder Richtung Norden und Westen, wo sie an einigen Stellen wieder aufeinandertrafen und Hybridzonen ausbildeten, die bis heute stabil sind (Barton und Hewitt 1985). Prominente Beispiele für Hybridzonen in Europa sind Aaskrähe (Corvus corone), Hausmaus (Mus domesticus und Mus musculus) und Unken (Bombina bombina und Bombina variegata). Aber auch Hybridzonen zwischen Heuschreckenarten bzw. Unterarten sind in den letzten Jahrzehnten intensiv erforscht worden. 


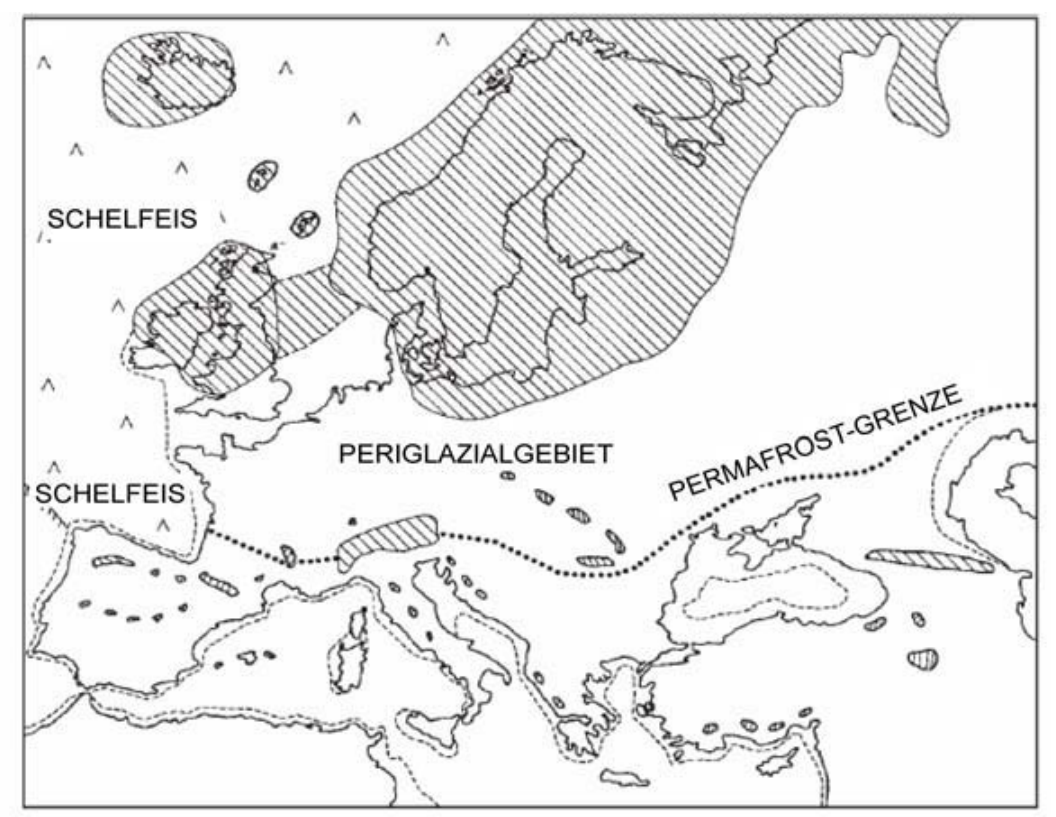

Abb. 1 Europa während der letzten maximalen Vereisung vor ca. 20.000 Jahren mit Ausdehnung der Gletscher (Schraffur), Küstenlinien (gestrichelte Linien) und Verlauf der Permafrostgrenze (punktierte Linie) (verändert nach Hewitt 1999).

\subsection{Orthopteren als Untersuchungsobjekt für Artbildungsprozesse}

Für die Untersuchung von Artbildungsprozessen sind Orthopteren besonders gut geeignet. Zusätzlich zu morphologischen und genetischen Markern können bei Orthopteren nämlich auch Verhaltensmerkmale herangezogen werden. Das Verhalten und speziell die intersexuelle Kommunikation zeichnet sich hier in vielen Fällen durch eine streng artspezifische Diversität aus. Neben Grillen (Ross und Harrison 2002; Howard 1986) zeigen vor allem die Männchen von Feldheuschrecken, die zur Unterfamilie der Gomphocerinae gehören, ausgeprägte artspezifische Gesänge. Während der Balz werden durch Stridulation der Hinterbeine an den Vorderflügeln die meist kompliziert aufgebauten und oft lang andauernden Gesänge erzeugt. In einigen wenigen Arten werden auch Lautsignale durch Flügelschlag während des Heuschreckengesangs verwendet (Elsner 1974b; Elsner und Wasser 1995a,b,c; Schütze und Elsner 2001). Manche Arten präsentieren zusätzlich zu den Lautäußerungen meist optische Signale durch rhythmische Bewegungen ihrer Antennen, der Palpen, des Kopfes, der Flügel oder des ganzen Körpers (Faber 1953, Jabobs 1953, Elsner 1974a, Helversen 1986, Ragge und Reynolds 1998). 
Bei diesen Feldheuschrecken kam es vor allem seit dem Holozän (vor ca. 12.000 Jahren) zu einer enorm großen Artenvielfalt, wobei sich die Spezies in erster Linie durch komplexe Lock- und Werbegesänge und den entsprechenden Weibchenpräferenzen unterscheiden.

Derartige intraspezifische Kommunikationssysteme, bestehend hier aus dem Werbeverhalten der Männchen (Sender) und der darauf abgestimmten Weibchenpräferenz (Empfänger) bilden in vielen Fällen eine hohe prägame Isolationsbarriere. In der Natur hybridisieren beispielsweise die beiden in vielen Gegenden sympatrisch verkommenden Heuschreckenarten Chorthippus biguttulus und Ch. mollis in der Regel nicht miteinander. Diese Arten sind sich anatomisch sehr ähnlich, unterscheiden sich aber in ihren Lautmustern stark voneinander, und daher lassen die Weibchen aufgrund des Werbeverhaltens der artfremden Männchen interspezifische Kopulationen nicht zu. Im Labor gelingt es aber, die beiden Arten miteinander zu kreuzen, wobei sich die Hybriden fruchtbar fortpflanzen können (Helversen und Helversen 1975a,b).

Obwohl selten, so gibt es aber in Europa an verschiedenen Stellen natürliche Heuschrecken-Hybridzonen, auf die im Folgenden eingegangen wird:

\subsection{Heuschrecken-Hybridzonen in Europa}

Bei Heuschrecken sind vor allem Hybridzonen innerhalb der phylogenetisch jungen Gattung Chorthippus ausgiebig erforscht worden. Vertreter dieser Heuscheckengattung trafen nach der letzten Kältezeit vor allem an Gebirgspässen der Pyrenäen und der Alpen aufeinander und bildeten dort meist schmale, aber langgezogene Hybridzonen. Entlang der Pyrenäen trafen die Unterarten Chorthippus parallelus parallelus und dessen südliche Unterart Ch. parallelus erythreus aufeinander (Hewitt 1993; Buño et al. 1994). Eine weitere Hybridzone ist zwischen Ch. brunneus und Ch. jakobsi ebenfalls in den Pyrenäen beschrieben worden (Bridle, Baird, Butlin 2001; Bailey, Thomas, Butlin 2004). Hybridzonen in den Alpen sind innerhalb der Gattung Chorthippus zwischen Ch. eisentrauti und Ch. brunneus sowie zwischen Ch. eisentaruti und Ch. biguttulus von Ingrisch (1995) näher untersucht worden. Ferner gibt es in Osteuropa eine über $200 \mathrm{~km}$ breite Hybridzone, in der die beiden Arten Ch. oschei und Ch. albomarginatus aufeinandertreffen und miteinander hybridisie- 
ren (Vedenina und Helversen 2003). In diesen Studien geht es neben der Charakterisierung der Elternarten sowie deren Hybriden in erster Linie um Selektionsmechanismen gegen Hybride, die so die Ausbreitung von Hybridzonen verhindern und daher die Elternarten stabilisieren. Der Balzgesang aber auch sterile F1 Hybriden gelten bei diesen Hybridzonen als die wichtigsten Isolationsbarrieren (Virdee und Hewitt 1992, 1994; Gottsberger und Mayer 2007). Trotz dieser heute bekannten relativ stabilen Hybridzonen gibt es auch einige Hinweise, dass Arten dieser Gattung, durch Hybridisierung entstanden sind (Vedenina et al. 2007). Es könnte zum Beispiel in Folge von genetischer Introgression durch Hybridisierung zur Bildung neuer Arten kommen (Jiggins et al. 1997, Jiggins und Mallet 2000, Seehausen 2004, Mallet 2005).

$\mathrm{Zu}$ den Arten der Gattung Chorthippus, die aller Wahrscheinlichkeit nach Produkt einer Artbildung durch Hybridisierung sind, gehören Ch. rubratibialis, Ch. jutlandica und Ch. karelini. Der Gesang von Ch. rubratibialis, die in Italien auf dem Apennin, vorkommt, besitzt Elemente der beiden Arten Ch. biguttulus und Ch. mollis (Schulze, pers. Mitt., siehe auch Elsner, Klöpfel und Sradnick 2009) .

Ch. jutlandica ist an der Westküste Jütlands (Dänemark) zu finden (Nielsen 2003). Gesangsanalysen zeigten, dass diese Art Elemente von Ch. biguttulus und Ch. brunneus beinhaltet und sehr stark Laborhybriden dieser beiden Arten ähnelt (Gottsberger 2008; siehe auch Elsner, Klöpfel und Sradnick 2009). Hinweise auf abgeschlossene Artbildung durch Hybridisierung gibt es bei der Art Ch. karelin, die aller Wahrscheinlichkeit nach ihre völlig neuartigen Gesangselemente aus Kombination der Gesänge von Ch. oschei und Ch. albomarginatus generiert hat (Vedenina und Helversen 2003).

\subsection{Inselpopulationen und deren Bedeutung bei der Artbildung}

Anders als die zuvor beschriebenen Individuen der Gattung Chorthippus ist die Verbreitung der in der vorliegenden Dissertation untersuchten Heuschreckenarten St. rubicundus und St. clavatus inselartig. Das heißt nicht, dass sie auf wirklichen Meeres-Inseln vorkommen, sondern - in Griechenland - auf voneinander inselartig getrennten Bergmassiven ab einer Höhe von $1.300 \mathrm{~m}$. 
Für die Entstehung neuer Arten ist eine geographische Isolation förderlich und oft sogar Ausgangspunkt für allopatrische Speziation (Mayr 1967). Am Beginn steht die Trennung der Habitate bestimmter Populationen voneinander. Diese kann durch geologische und ökologische Veränderung hervorgerufen werden. Typische Beispiele für „Inseln“ sind ozeanische Inseln, Berggipfel, Seen oder Wüstenoasen, aber auch Stadtparks und oder kleine Wälder, umgeben von Agrarlandschaften. Bekannte Beispiele für Speziationsprozesse infolge geographischer Isolation sind unter anderem die Galápagos-Finken (Grant und Grant 1996a,b; 1998, Grant et al. 2005), die Buntbarsche Malawis (Meyer 1989, Rüber et al. 1998; Genner et al. 2004, Seehausen 2006, Maderbacher et al. 2008 ) und Heuschrecken der Gattung Melanopus der Rockymoutains in Nordamerika. Grant und Grant (1996a,b) beschrieben bei von Galapagos-Finken eine gesteigerte Fitness von Hybriden gegenüber ihren Elternarten in durch Klimaschwankungen hervorgerufene sehr dezimierten Populationen. Weiter wird angenommen, dass besonders allopatrische Artbildung durch Hybridisierung begünstigt werden kann (Kirkpatrick und Servedio 1999, Hewitt 2001).

Vor allem werden noch viele Diskussionen über den geographischen Modus von Artbildung geführt. Dabei ist die Bedeutung von allopatrischer Artbildung aufgrund genetischer Drift und/oder Selektionsprozesse wodurch reproduktive Isolation als Nebenprodukt entsteht (Mayr 1963), weiterhin umstritten.

Die Speziationsprozesse, die zum enormen Artenreichtum der heutigen europäischen Orthopteren geführt haben sind zu großen Teilen ungeklärt. Geographisch isolierte Populationen („Inselpopulaitonen oder interglaciale Refugien) zeichnen sich durch große Artenvielflat und eine große Anzahl von Endemiten aus. In isolierten Populationen herrschen meist besonderen ökologische Bedingungen und evolutive Prozesse wie Immigration von elterlichen Genotypen in das Zentrum der Hybridzone, Genaustausch zwischen den Populationen und die Hybridisierungsrate könnten im Vergleich zu nicht isolierten Populationen anders sein. In isolierten Populationen können Fragestellungen der allopatrischen Artbildung aber auch der Einfluss von Hybridisierung bei der Speziation untersucht werden.

Die bereits (Sradnick 2006) beschriebene Hybridzone zwischen St. rubicundus und St. clavatus ist im Vergleich zu anderen Hybridzonen besonders klein und geographisch isoliert. Sie bietet sich somit besonders gut an um evolutionsbiologische Fragen zu klären. 


\subsubsection{Hybridzone auf dem Tomaros}

Die in dieser Arbeit zu untersuchende Hybridzone ist ebenfalls geographisch isoliert. Es handelt sich um eine aus zwei der Gattung Stenobothrus zugehörigen Arten.

Die beiden Arten, Stenobothrus rubicundus und Stenobothrus clavatus, haben eine inselartige Verbreitung und kommen in Griechenland ausschließlich in Höhenlagen oberhalb von $1300 \mathrm{~m}$ vor (Abb. 2). Zudem ist eine der beiden Arten (St. clavatus) endemisch auf dem Xerovouni und dem Tomarosgebirge.

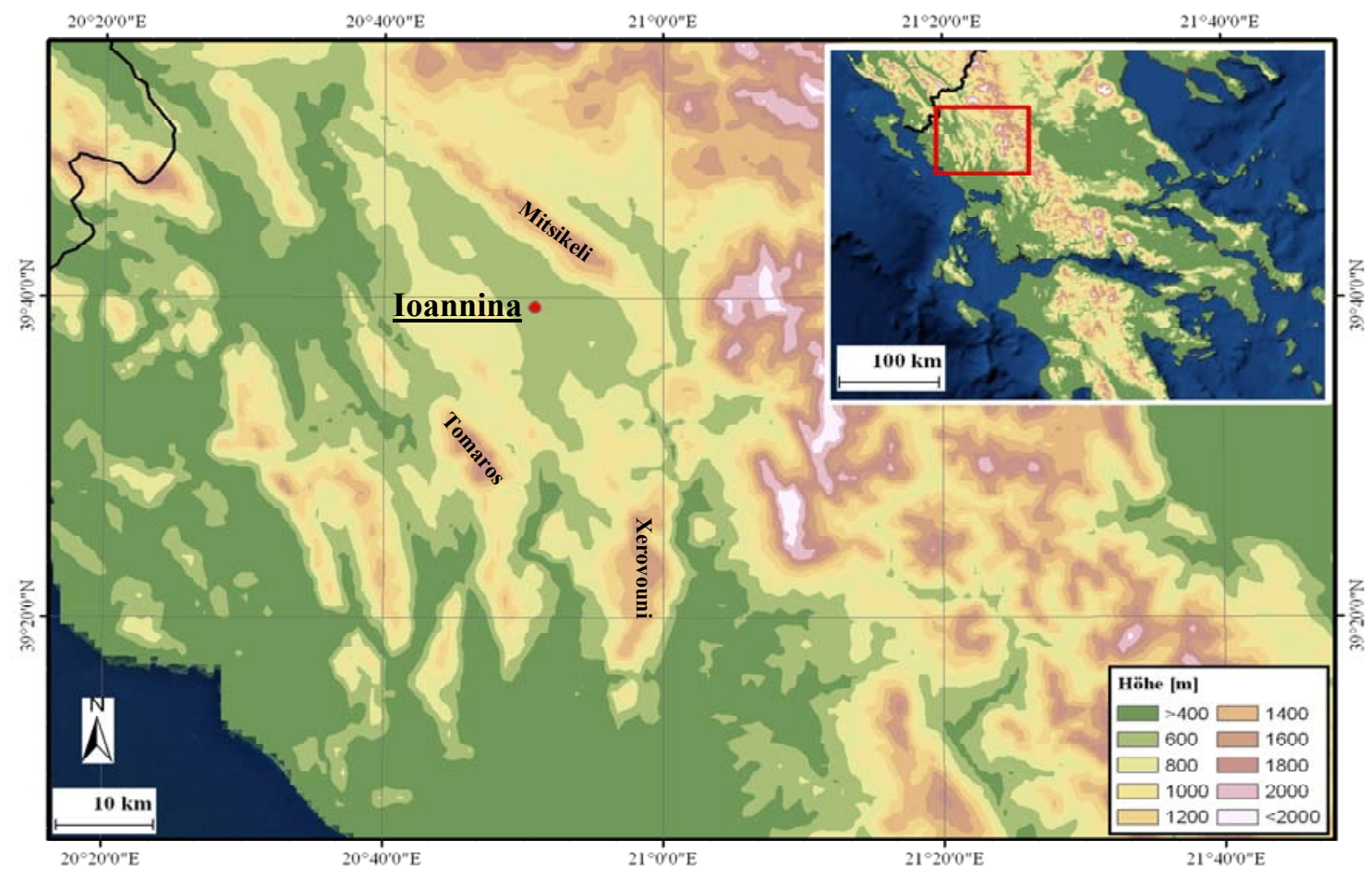

Abb. 2 Höhenprofil des Untersuchungsbietes im Nordwesten Griechenlands. Zu sehen ist der Tomaros, auf dem sich die Hybridzone befindet, sowie die Berge der allopatrischen Vergleichspopulationen von St. rubicundus (Mitsikeli) und St. clavatus (Xerovouni).

\subsection{Versuchstiere}

St. rubicundus Germar $1817^{1}$ ist vor allem auf der Balkanhalbinsel und in den Alpen weit verbreitet (Harz 1957, Bellmann 1993, Ragge und Reynolds 1998), tritt aber überall nur an vereinzelten und weit auseinander liegenden Fundorten auf. Dieses Verbreitungsmuster ist als inselartig zu bezeichnen. 
Wichtige morphologische Merkmale sind die geraden unverbreiterten Antennen, die großen stark sklerotisierten Vorder- und Hinterflügel sowie das mittig auf dem Vorderflügel zu findende Stigma (heller Fleck auf dem Flügel). Das Abdomen der Männchen ist auffällig rot gefärbt (Abb. 5); daher auch die Namensgebung ,rubicundus“(lat: rötlich). Während der Balz, die in 3.4.1 noch näher beschrieben wird, machen sie durch lautes Flügelschnarren auf sich aufmerksam (Abb. 3).

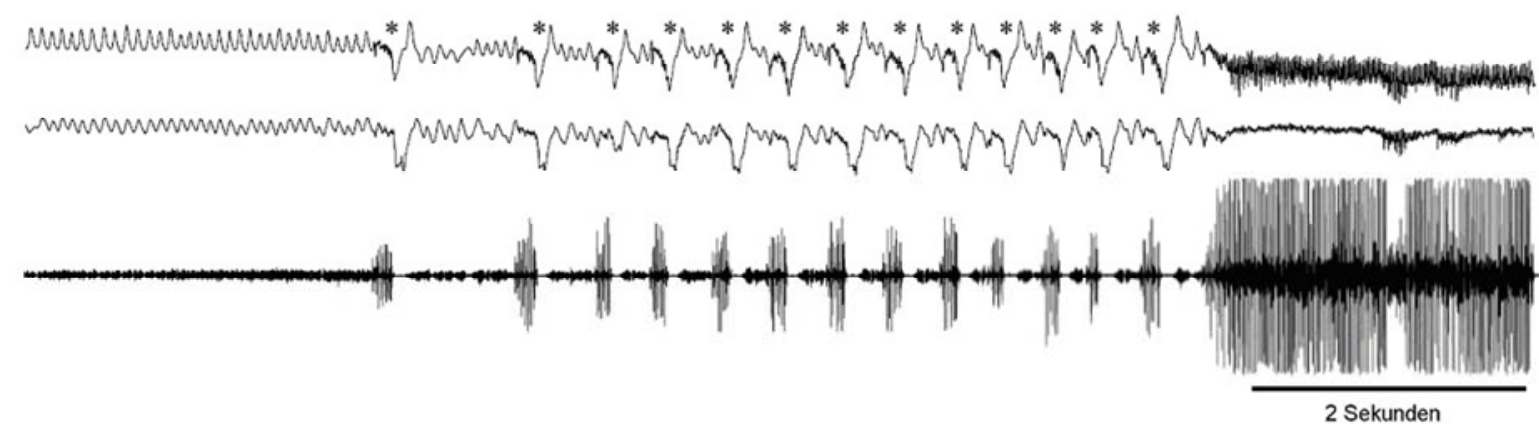

Abb. 3 Balz eines Männchens von Stenobothrus rubicundus. Untere Spur: Laute; obere Spuren: Registrierungen der Stridulationsbewegungen, wobei die Flügelbewegungen $(*)$ indirekt durch Ab- schattung der Beine erfasst werden (siehe Elsner und Wasser 1995b). Aufgenommen mit Positionsdetektoren.

Im Gegensatz zu St. rubicundus ${ }^{1}$ ist Stenobothrus clavatus Willemse 1979 eine endemische Art, die nur auf zwei Bergen Nordwestgriechenlands, dem Xerovouni und dem Tomaros, in der Nähe der Stadt Ioannina vorkommt. Ein auffälliges Merkmal dieser Art sind die verbreiterten rechtwinklig nach unten gebogenen Antennenenden (Abb. 5). Diese morphologische Eigenschaft stand Pate für den Artnamen (lat: clavator = der Keulenträger). Im Gegensatz zu St. rubicundus sind die Vorder- und Hinterflügel von St. clavatus wenig sklerotisiert, um einiges kürzer und reichen nicht über das Abdomen hinaus. Die Balz der Männchen (Abb. 4) ist sehr Komplex beinhaltet aber kein Flügelschnarren sondern auffällige hochamplitudige Bein und Antennenbewegungen (siehe Kapitel 3.4.2).

\footnotetext{
${ }^{1}$ Stenobothrus rubicundus Germar 1817: Synonyme: St. miniatus Charpentier 1825, St. Crotalacris rubicundus Chopard 1951 und St. rubicundulus Kruseman und Jeekel 1967. Der Argumentation von Kruseman und Jeekel (1967), der Name St. rubicundus müsse geändert werden, weil Germars Beschreibung inkorrekt sei, wird nicht gefolgt, da zum einen dieser Name seit mehr als 60 Jahren in Gebrauch ist (siehe z. B. Jacobsen und Bianchi 1902) und zum anderen durch Chopard (1951) noch einmal eindeutig klargestellt wurde, um welches Tier es sich handelt.
} 


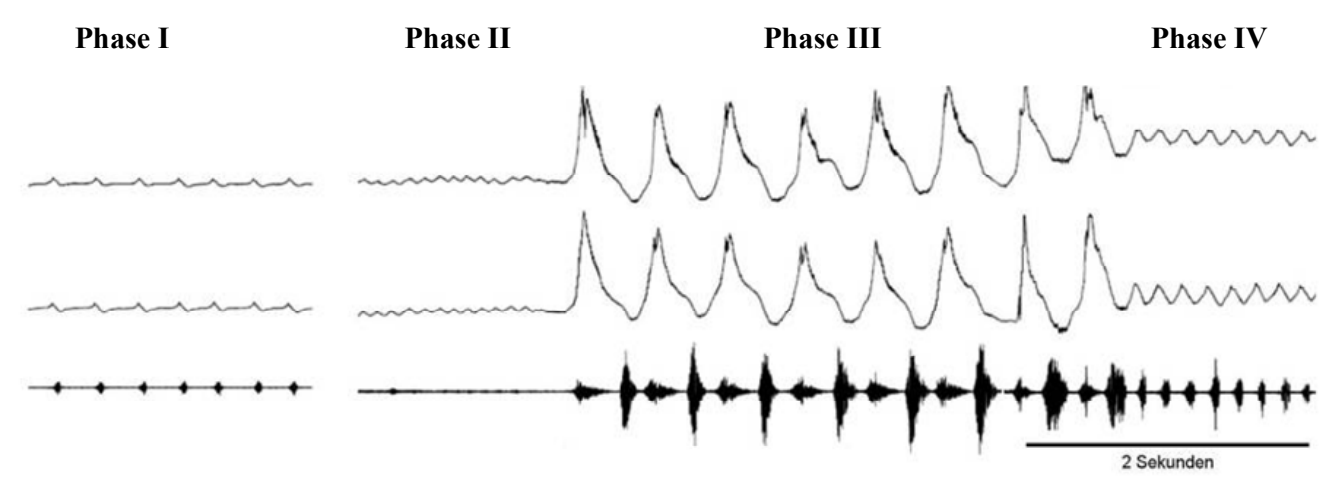

Abb. 4 Balz eines Männchens von Stenobothrus clavatus. Untere Spur: Laute; obere Spuren: Registrierungen der Stridulationsbewegungen. Aufgenommen mit Positionsdetektoren.
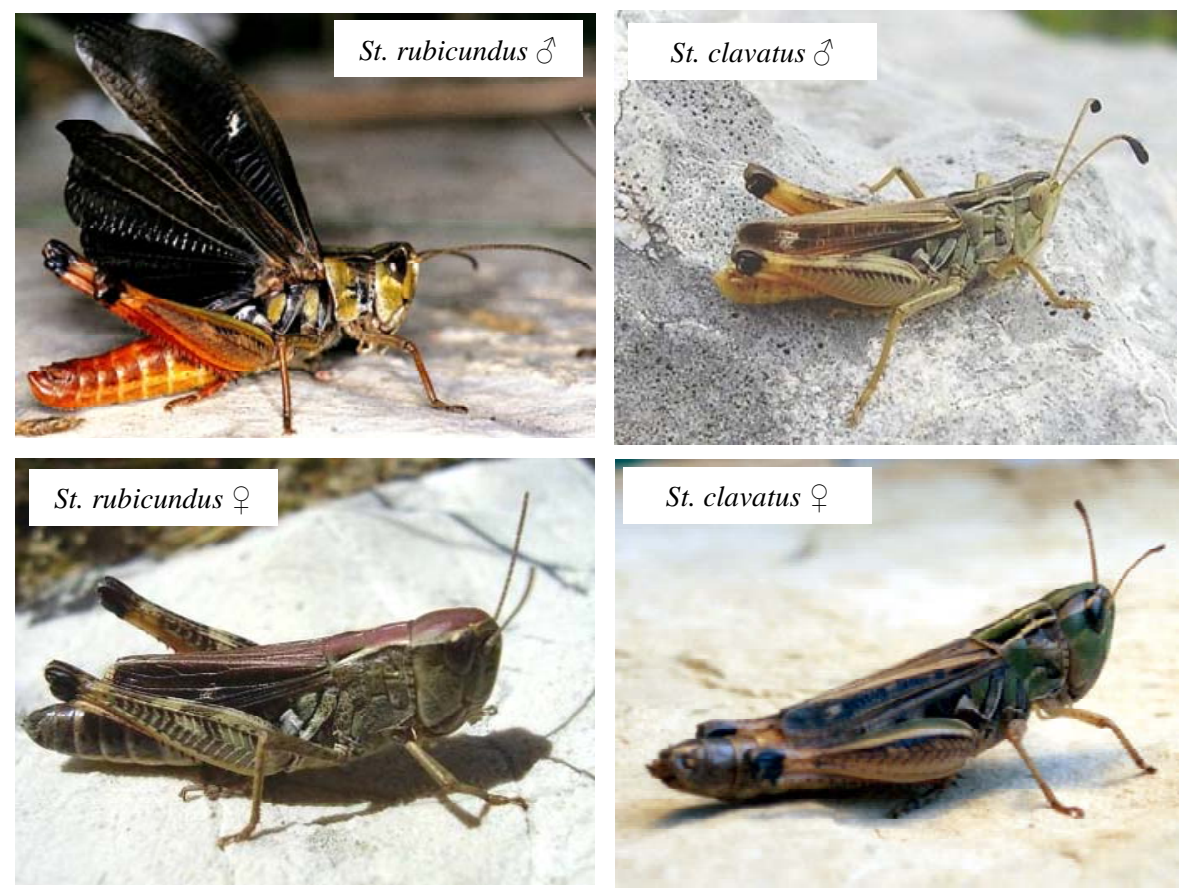

Abb. 5 Männchen (oben) und Weibchen (unten) der beiden Arten St. rubicundus (links) St. clavatus (rechts).

Bis zum Beginn der Arbeit an der vorliegenden Dissertation war St. clavatus lediglich auf dem Südhang des Tomaros-Südmassivs gefunden worden (Willemse 1979). Im Jahre 1986 fand Dr. Helmut Kriegbaum (pers. Mitteilung) erste Hinweise auf eine Hybridzone zwischen St. rubicundus und St. clavatus auf dem Tomaros, wobei aber die Existenz einer St. rubicundus-Elternpopulation zunächst nicht nachgewiesen werden konnte. Auch nach weiteren Exkursionen von F. Willemse, L. Willemse und N. Elsner (pers. Mitteilungen) blieb vorerst unklar, ob es sich tatsächlich um Hybriden oder nur um unterschiedliche morphologische Varianten von St. clavatus handelt. Erst im Jahr 2005 konnte durch faunistische Erkundungen und verhaltensbiologische sowie morphologische Untersuchungen gezeigt werden, dass auf dem Tomaros tatsächlich Hybriden zwischen St. rubicundus und St. clavatus sowie die beiden Elternarten vorkommen (Sradnick 2006). Ferner wurde im Zuge der 
Arbeit an der vorliegenden Dissertation eine Population von St. clavatus auf dem Xerovouni-Gebirge entdeckt, wo St. rubicundus hingegen nicht vorkommt.

\subsection{Ziel der Arbeit}

Ein wesentliches Ziel der vorliegenden Dissertation ist es, die Populationen der beiden Arten St. rubicundus und St. clavatus sowie deren Hybriden auf dem Tomaros-Gebirge zu kartieren und morphologisch sowie verhaltensbiologisch $\mathrm{zu}$ charakterisieren. Ferner soll geklärt werden, welche Struktur die Hybridzone hat. Hierzu sind die Tomaros-Tiere mit Individuen allopatrischer Population von St. rubicundus und St. clavatus zu vergleichen, die keinen Kontakt zur jeweils anderen Art haben. Diese stehen im Falle von St. rubicundus auf mehreren Bergen Griechenlands, Mitsikeli, Vernon, Valtou etc. zur Verfügung. Eine Vergleichspopulation von St. clavatus findet sich auf dem Xerovouni-Gebirge. In diesem Zusammenhang soll herausgefunden werden, wie es zur allopatrischen Trennung der Vergleichspoplautionen und zur parapatrischen Trennung auf dem Tomaros kommt.

Die Feldhybride sollen morphologisch und ethologisch charakterisiert werden, um diese dann mit Laborhybriden zu vergleichen. Insbesondere die Merkmalsvielfalt und augenscheinliche Fitness der Hybriden soll mögliche Hinweise auf Selektion gegen Hybride geben. In diesem Zusammenhang soll untersucht werden, ob prä- und postgamen Isolationsbarrieren wirksam sind. Ebenfalls sollen die Mechanismen charakterisiert werden, die zur Hybridisierung führen und somit Isolationsbarrieren überwinden. Zusätzlich werden durch Vergleiche zwischen Labor- und Feldhybriden Evolutionsmechanismen betrachtet, die einen Beitrag zur Hybridisierung der Arten leisten. Des Weiteren sollen die Populationen auf dem Tomaros auf Anzeichen von Genaustausch in Folge der Hybridisierung hin untersucht werden. Dies wird in Zusammenhang der besonderen inselartigen Populationsstruktur der untersuchten Populationen gestellt. Dabei geht es vor allem um den möglichen Beitrag von Hybridzonen zur Speziation in einem interglacialen Refugium wie Griechenland. 


\section{Material und Methoden}

\subsection{Vorkommen der zum Vergleich herangezogenen Populationen von} St. rubicundus und St. clavatus

Von den in dieser Arbeit untersuchten beiden Arten kommt Stenobothrus rubicundus in der Regel oberhalb von 1000 m an zahlreichen Standorten in den Alpen, auf dem Apennin und den Bergen des Balkans vor (Ragge und Renolds 1998). Stenobothrus clavatus hingegen war bisher nur vom Tomaros-Gebirge bekannt (Willemse 1979); das Vorkommen auf dem Xerovouni-Gebirge wurde erst 2007 entdeckt (Sradnick und Klöpfel, unveröffentlicht). Im Folgenden werden die für die vorliegende Dissertation relevanten Populationen und Standorte (Abb. 6) näher beschrieben.

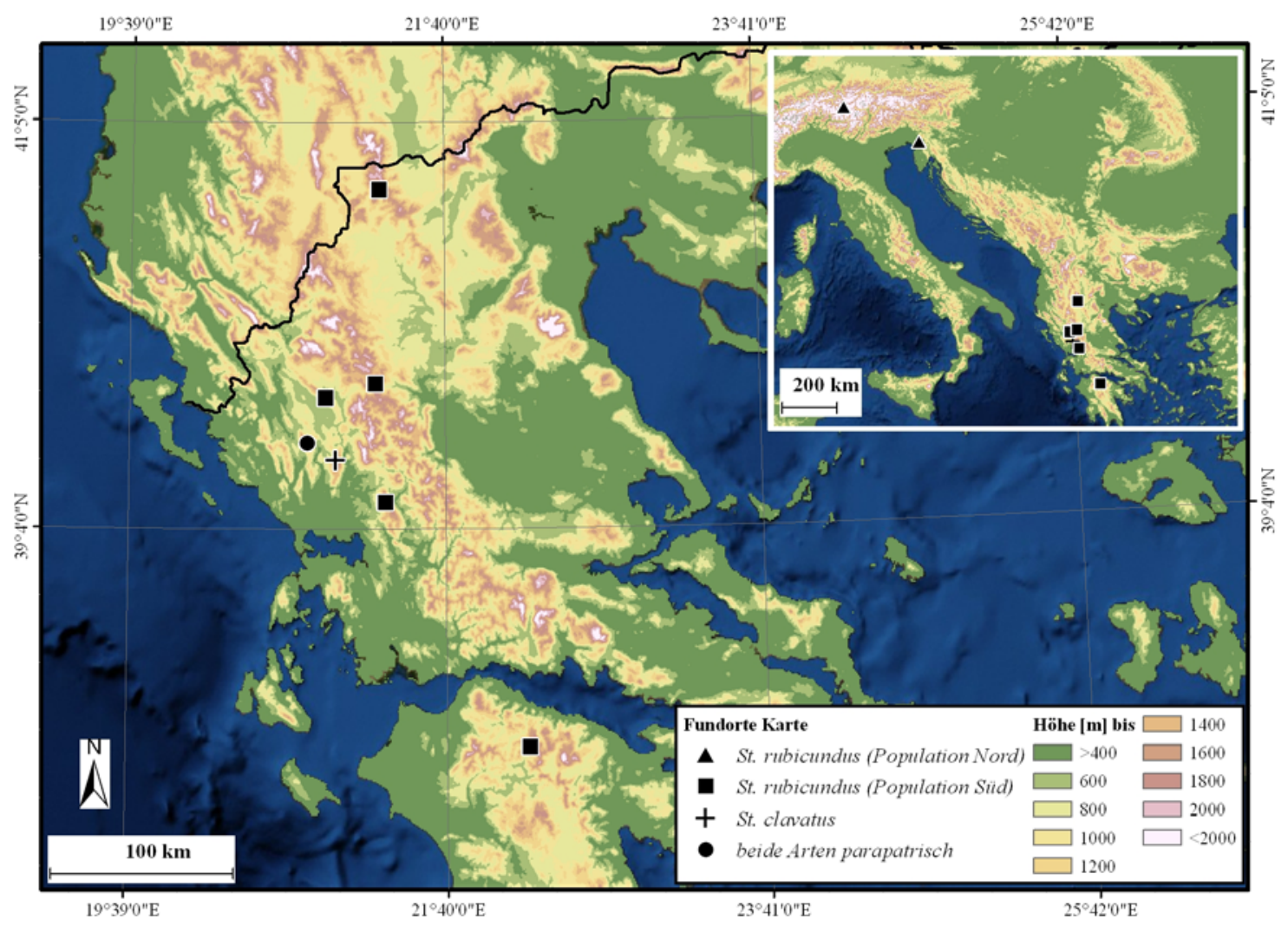

Abb. 6 Karte der, in der vorliegenden Arbeit ausgewählten, Fundorte der Arten St. rubicundus und St. clavatus in Griechenland (große Karte) und Südeuropa (kleine Karte).

\subsubsection{Stenobothrus rubicundus}

Stenobothrus rubicundus kommt hauptsächlich im Hochgebirge vor. Das Ausbreitungsgebiet erstreckt sich von den Alpen über den Balkan bis hin zum südlichsten Teil der griechi- 
schen Halbinsel Peloponnes (Abb. 6). In den Alpen findet man diese Art ab einer Höhe von ca. $1.000 \mathrm{~m}$, vereinzelt auch schon ab $800 \mathrm{~m}$. Ähnlich ist es in den Dinarischen Gebirgen des Balkans, wobei nach Süden hin die untere Habitatsgrenze ansteigt. Im nördlichen Griechenland liegt sie bei ca. $1300 \mathrm{~m}$ und auf den Bergen der Peloponnes bei $1600 \mathrm{~m}$. Eine Ausnahme hierzu bildet eine kleine Population im westlichen Slowenien (nahe Komen). In diesem Gebiet ist St. rubicundus schon in einer Höhe von $50 \mathrm{~m}$ bis $200 \mathrm{~m}$ über dem Meeresspiegel zu finden. Bis auf diese Ausnahme kommt die Art jedoch ausschließlich auf hohen Bergen, also montan vor. So ergibt sich eine inselartige Ausbreitung, die eng mit den geographischen Gegebenheiten verknüpft ist.

Im Rahmen der vorliegenden Arbeit sind, abgesehen von den Tomaros-Populationen hauptsächlich sieben weitere St. rubicundus-Populationen untersucht und für Vergleichszwecke herangezogen worden. Dies sind die alpine Population im oberen Engadin (Schweiz), die eben erwähnte slowenische Population, ferner eine im äußersten Norden Griechenlands im Vernongebirge, außerdem zwei kleinere Populationen nahe Ioannina auf dem Mitsikeli- und dem Valtou-Gebirge sowie eine Population im Aroania Gebirge der Peloponnes auf dem Berg Helmos. In den folgenden Abschnitten werden diese St. rubicundus Habitate hinsichtlich Geographie und Ökologie beschrieben, wobei auf die Tomaros-Populationen erst später ausführlich eingegangen wird.

\subsubsection{Alpine und Slowenische Population}

Für Vergleichszwecke wurden einige Tiere aus dem oberen Engadin herangezogen, wo St. rubicundus in einer Höhe von $1.200 \mathrm{~m}$ bei S-chanf sowie ab $2000 \mathrm{~m}$ oberhalb von Pontresina vorkommt. St. rubicundus kommt oberhalb der Ortschaft an steinigen Hängen die mit Festuca bewachsen sind, vor.

Nahe des, an der Westgrenze Slowenien gelegenen, Dorfes Brje pri Komnu findet man St. rubicundus vor allem auf Wiesen mit dichter Vegetation, die lediglich $150 \mathrm{~m}$ über den Meeresspiegel liegen (Abb. 7). Eine der prominentesten Gräsergattungen in diesem St. rubicundus-Habitat ist die Gattung Festuca. Diese Festuca-Wiesen zeichnen sich durch einen hohen Artenreichtum aus und stehen somit im Kontrast zu den kargen Habitaten der alpinen und montanen Populationen. 


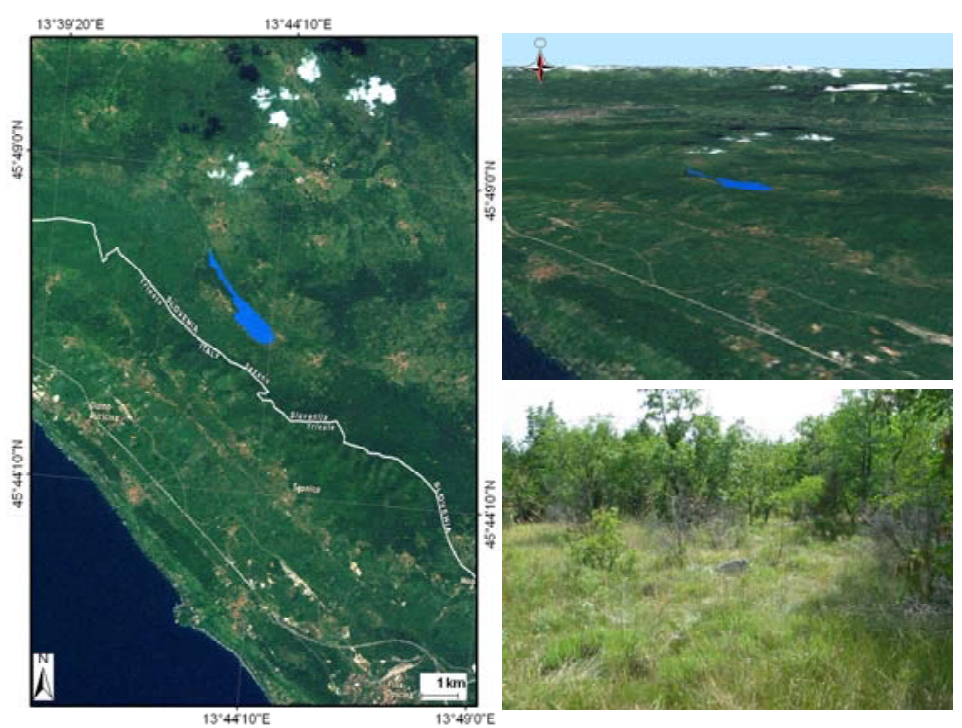

Abb. 7 Ausbreitungsgebiet (blaue Fläche) der St. rubicundus-Population nahe der südwestslowenischen Ortschaft Brje pri Komnu. Sattelitenbild (links), dreidimensionale Ansicht (oben-rechts) und Foto des Habitats (unten-links).

\subsubsection{Vernon-Gebirge}

Im äußersten Nordosten Griechenlands, in der Region Westmakedonien zwischen der Stadt Florina den Prespa-Seen liegt das Vernon-Gebirge (Abb. 8), das sich über 20 - 25 km von Südwesten nach Nordosten und etwa 25 - $30 \mathrm{~km}$ von Nordwesten nach Südosten erstreckt und dessen höchste Erhebung mit $2.128 \mathrm{~m}$ der Vitsi ist. St. rubicundus kommt hier nahe des Pissoderi-Passes in einer Höhe von ca. $1800 \mathrm{~m}$ in hohem Festuca-Gras auf Wiesen vor, die regelmäßig von Schafen beweidet werden und von Buchenwäldern umgrenzt sind.
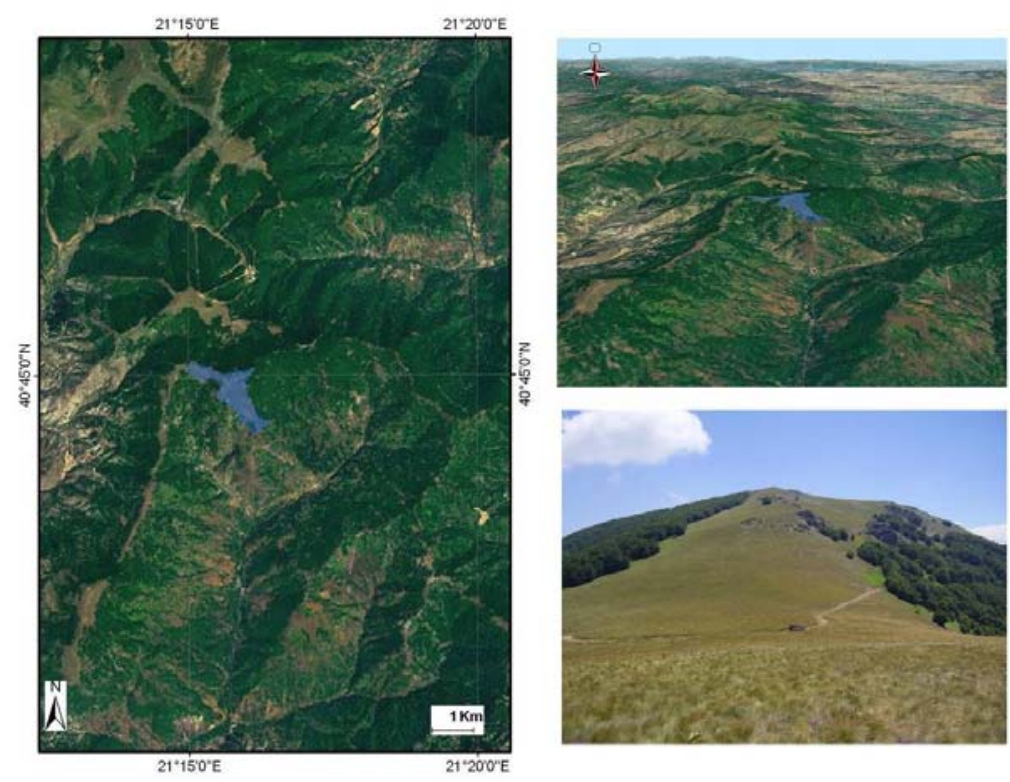

Abb. 8 Ausbreitungsgebiet der St. rubicundus-Population (blaue Fläche) des Vernongebirges. Sattelitenbild (links), dreidimensionale Ansicht (oben-rechts) und Foto des Habitats (unten-links). 


\subsubsection{Metsovo-Population}

Ein weiterer Fundort mit etwas anderem ökologischen Charakter liegt im Pindosgebirge, $14 \mathrm{~km}$ östlich von der Stadt Metsovo entfernt (Abb. 9). Hier findet man St. rubicundus in einer Höhe von 1720 bis 1770 Metern in einem lichten Kiefernwald, merkwürdigerweise aber nicht auf den kleinen frei liegenden Wiesen in unmittelbarer Nähe. Somit grenzt sich dieses Habitat von den anderen Fundorten, die eher einen wiesenartigen Charakter haben, ab. Insgesamt ist dieses St. rubicundus-Habitat im Vergleich zu den anderen Fundorten sehr dünn besiedelt.

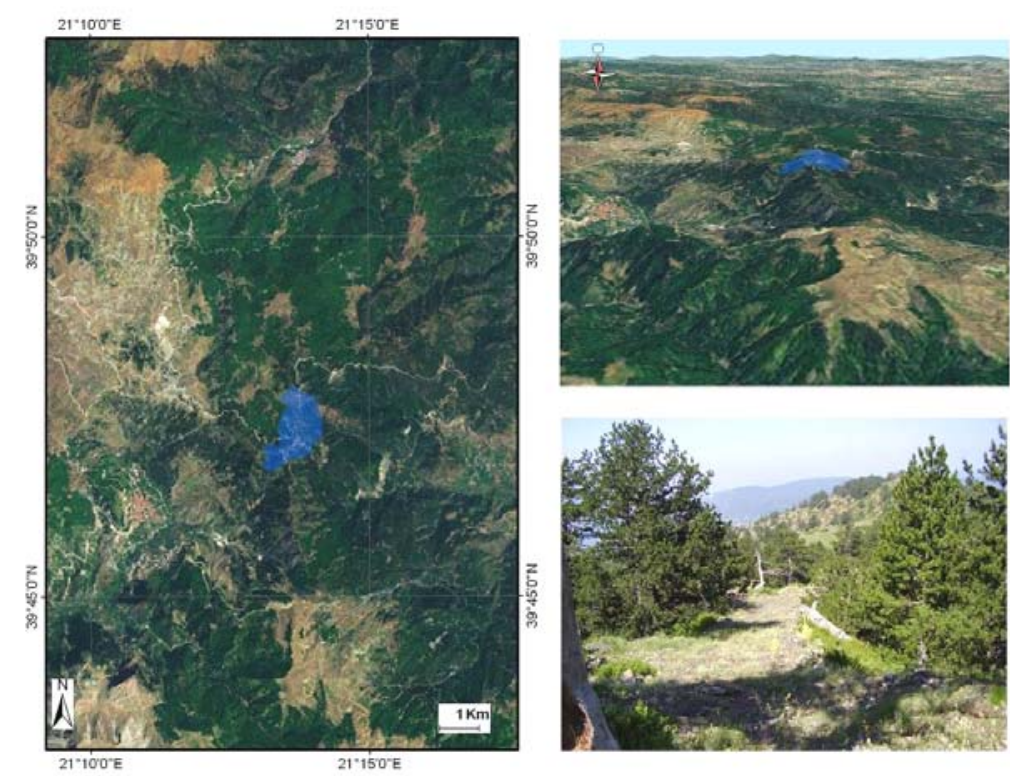

Abb. 9 Ausbreitungsgebiet der St. rubicundus-Population (blaue Fäche) in der Nähe von Metsovo. Sattelitenbild (links), dreidimensionale Ansicht (oben-rechts) und Foto des Habitats (unten-links).

\subsubsection{Mitsikeli-Population}

Nahe Ioannina, der Hauptstadt der griechischen Verwaltungsregion Epirus, erstreckt sich oberhalb des nördlichen Ufers des Pamvotida-Sees (Ioannina-See) das zum Pindosgebirge gehörige Mitsikeli-Massiv (Abb. 10). Am höchsten Punkt misst das Massiv 1810 m. Weitere fünf kleine Gipfel folgen auf insgesamt 17 Kilometer in nordwestlicher Richtung wobei in der Ost-West-Ausdehnung das Massiv mit 5 Kilometern sehr viel schmaler ist. Die Hänge des Massivs sind mit den für diese Gegend typischen Nadelbäumen bewachsen. Ab einer Höhe von 1300 m bis zum Gipfel erstreckt sich das für St. rubicundus typische unbewaldete, karge, mit Festuca bewachsene Habitat. Hier wird, wie auch im Vernon, die Fläche landwirtschaftlich genutzt; vor allem Rinder beweiden die Wiesen. Die Populati- 
onsdichte ist im Vergleich zur Vernon-Population geringer, übersteigt jedoch deutlich die des Fundortes nahe Metsovo.
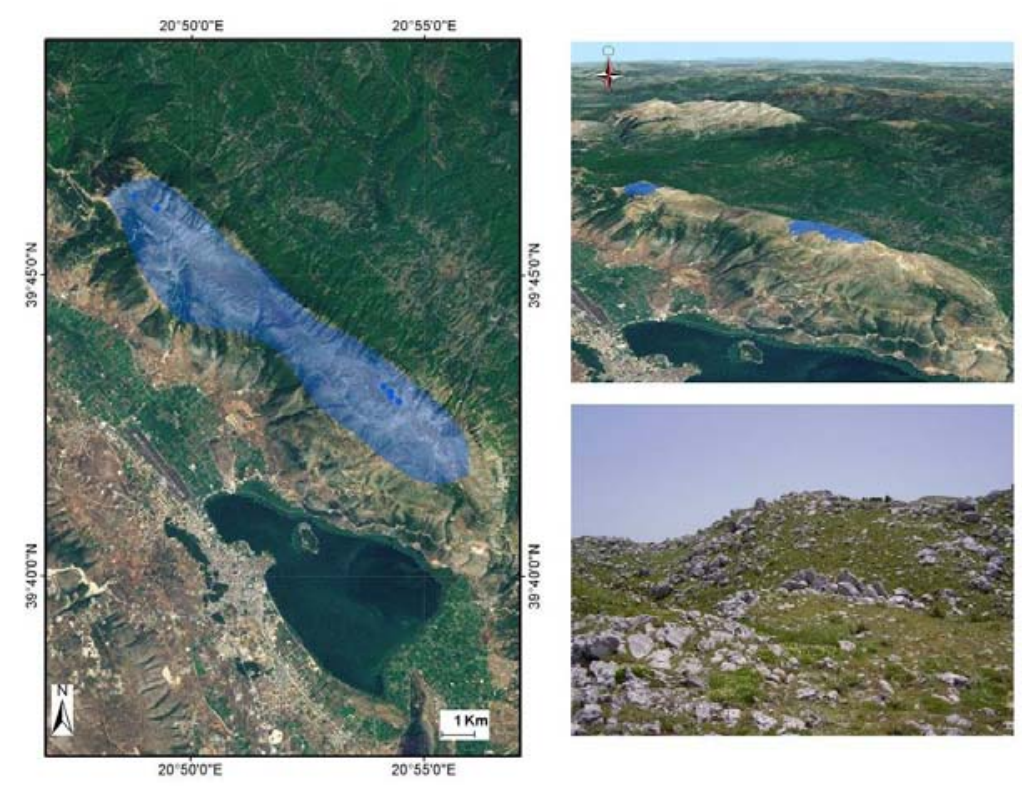

Abb. 10 Vermutetes Ausbreitungsgebiet (blaue Fläche) der Art St. rubicundus auf dem Mitsikeli. Sattelitenbild (links), dreidimensionale Ansicht (oben-rechts) und Foto des Habitats (unten-links).

\subsubsection{Valtou-Population}

Die ebenfalls zum Pindosgebirge gehörenden Ori Valtou (Valtou-Berge), liegen ca. 25 Kilometer Luftlinie östlich von der griechischen Stadt Arta entfernt (Abb. 11). Auf einer Gesamtlänge von 15 Kilometer erstreckt sich das Massiv in seiner Nord-Süd-Ausdehnung. Die Ost-West-Ausdehnung des Massivs ist hingegen maximal $5 \mathrm{~km}$ breit. Der höchste Gipfel ist der Aetoi mit 1781m. Die Hänge sind meist bis zu einer Höhe von 1000m bewaldet.

St. rubicundus wurde auf dem nördlichsten Gipfel in einer Höhe von 1758m gefunden. Das von Rindern beweidete Habitat ist karg und unbewaldet und, ähnlich wie bei den anderen Fundplätzen, ebenfalls mit Gräsern der Gattung Festuca bewachsen. Die Populationsdichte von St. rubicundus ist deutlich geringer als im Vernon-Gebirge. 

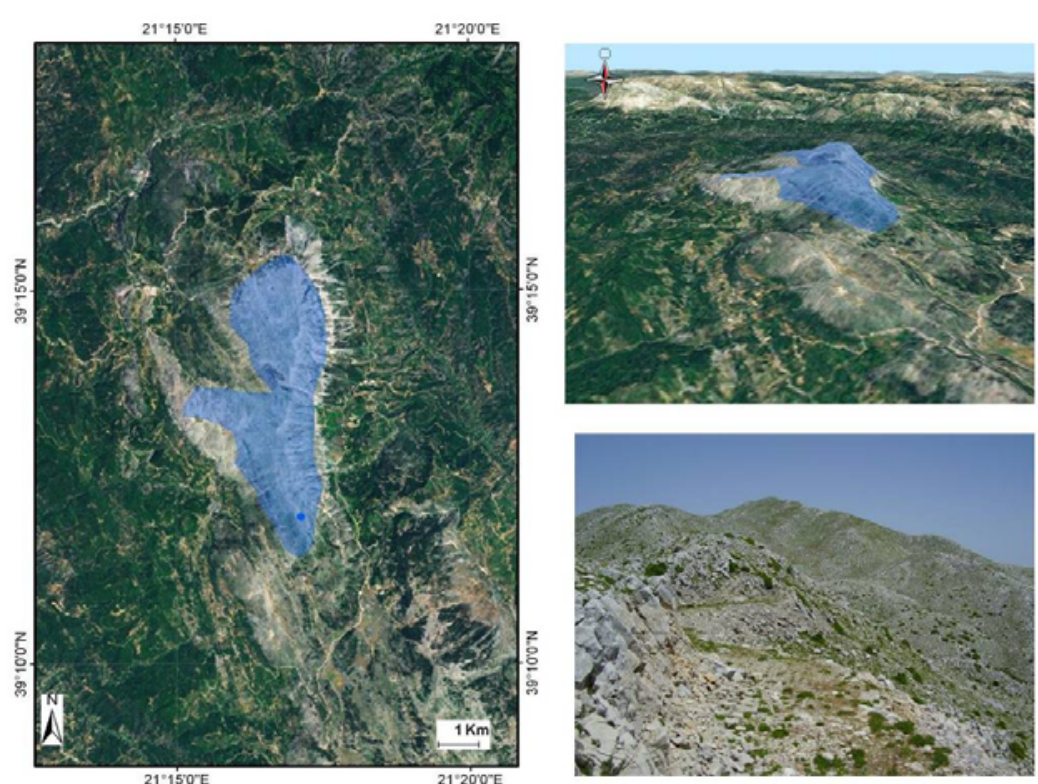

Abb. 11 Vermutetes Ausbreitungsgebiet (blaue Fläche) der Art St. rubicundus im Vernon-Gebirge. Sattelitenbild (links), dreidimensionale Ansicht (oben-rechts) und Foto des Habitats (unten-links).

\subsubsection{Peloponnes-Population}

Auf der Halbinsel Peloponnes kommt St. rubicundus an mehreren Standorten vor (Willemse 1985). Im Rahmen der vorliegenden Arbeit wurden Tiere vom Aroania-Massiv, 20 km vom griechischen Dorfes Kalavrita entfernt, herangezogen (Abb. 12). Hier kommen sie in einer Höhe von 2000 m unterhalb des Gipfels Helmos (2341 m) vor. Bei dieser Fundstelle handelt es sich um einen steilen Hang, der größtenteils aus losem Geröll und kleineren, inselartigen Grasmatten besteht, die von St. rubicundus besiedelt werden. Auf dem Gipfel selbst sind keine St. rubicundus zu finden. Ein weiterer Fundplatz befindet sich in einer Höhe von 1600 m auf einer Wiese nahe der Talstation eines Skiliftes. Wie auch auf den bereits beschriebenen Bergen bestimmen Gräser der Gattung Festuca die Hauptvegetation der St. rubicundus-Habitate. 


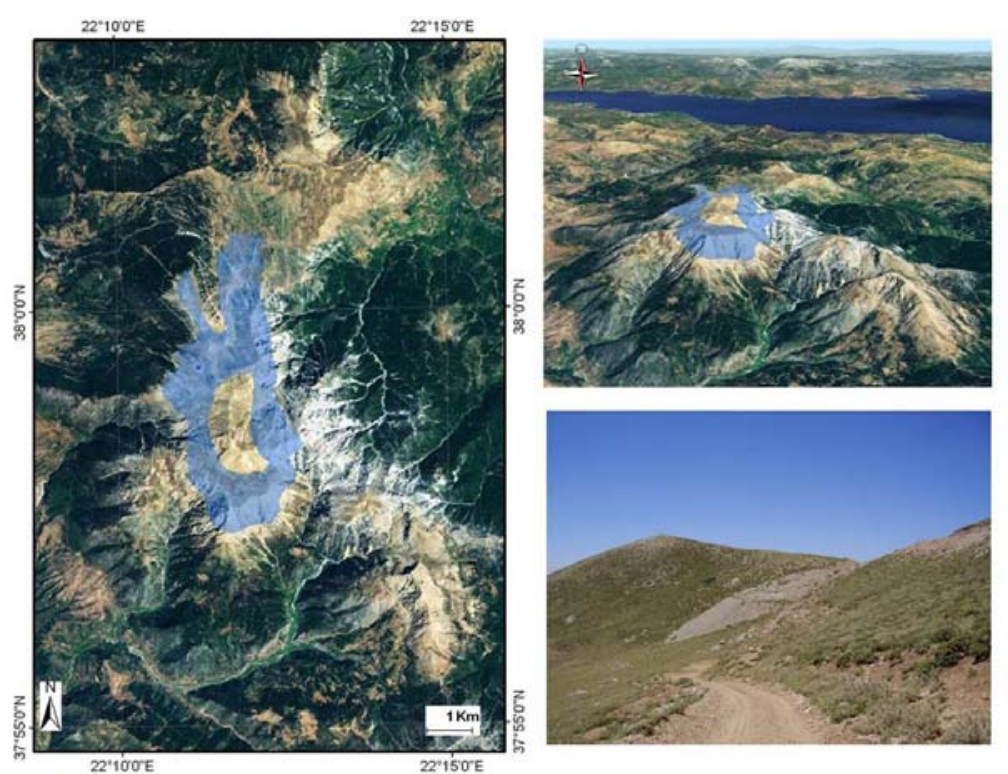

Abb. 12 Vermutetes Ausbreitungsgebiet (blaue Fläche) der Art St. rubicundus Helmos (Peloponnes). Sattelitenbild (links), dreidimensionale Ansicht (oben-rechts) und Foto des Habitats (unten-links).

\subsubsection{Stenobothrus clavatus}

Anders als St. rubicundus ist Stenobothrus clavatus ausschließlich auf den zwei benachbarten Bergen Tomaros und Xerovouni im Pindosgebirge in Höhen zwischen $1.300 \mathrm{~m}$ und $1.800 \mathrm{~m}$ - nicht darüber - zu finden. Diese beiden Fundorte befinden sich in der westgriechischen Provinz Epirus nahe der Stadt Ioannina. Andere Fundorte für diese Art sind nicht bekannt, und trotz intensiver Suche konnte sie auf weiteren Bergen des Epirus, wie z. B. Soulion, Valtou und Mitsikeli, nicht gefunden werden. Das Habitat von St. clavatus ist äußerst karg und unbewaldet.

\subsubsection{Xerovouni-Population}

Das maximal $1.641 \mathrm{~m}$ hohe Bergmassiv erstreckt sich in Nord-Süd-Richtung ca. 20-25 km; die Ost-West-Ausdehnung beträgt 10 bis $15 \mathrm{~km}$. Die Hänge verlaufen steil bis auf ca. 1100 $\mathrm{m}$ und gehen $\mathrm{ab}$ dieser Höhe in ein $27,3 \mathrm{~km}^{2}$ großes Plateau über, das dann langsam auf etwa $1600 \mathrm{~m}$ ansteigt. St. clavatus, der bisher nur vom Tomaros-Gebirge bekannt war, wurde im Jahre 2007 auf dem Xerovouni (Abb. 13) in einer Höhe ab 1300 m von unserer Arbeitsgruppe entdeckt (Sradnick und Klöpfel unveröffentlicht). Eingehende Erkundungen haben inzwischen gezeigt, dass hier ausschließlich St. clavatus, nicht aber St. rubicundus zu finden ist. 
Karge Wiesen, die mit lockerem Kalkstein und vereinzelten Festuca-Horsten übersät sind, bestimmen hier das Landschaftsbild. Somit unterscheidet sich das Habitat deutlich von den St. rubicundus-Habitaten, obwohl es, wie bei diesen, hauptsächlich von Gräsern der Gattung Festuca bestimmt wird. Auf diesem steinigen und hügeligen Bergrücken kommt St. clavatus fast flächendeckend vor und bildet so die größte zusammenhängende Population dieser Art.

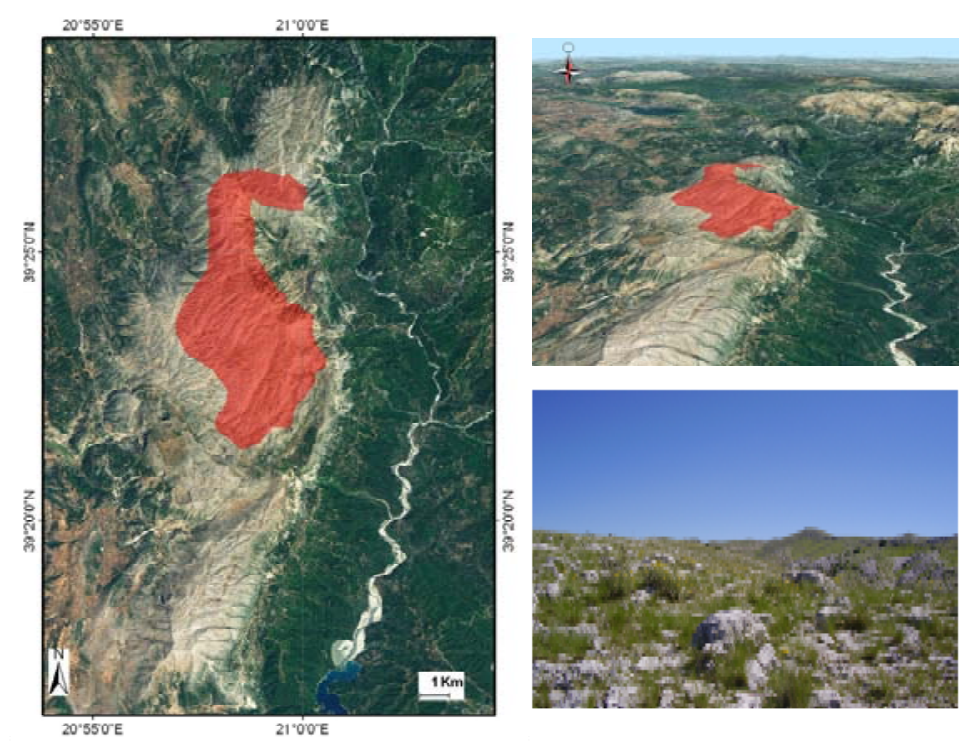

Abb. 13 Vermutetes Habitat (rote Fläche) der Art St. clavatus des Xerovouni. Sattelitenbild (links), dreidimensionale Ansicht (oben-rechts) und Foto des Habitats (unten-links).

\subsection{Tomaros-Populationen von St. rubicundus, St. clavatus und deren Hybriden}

Das Tomarosgebirge liegt ca. $20 \mathrm{~km}$ südlich von Ioannina und erstreckt sich mit Gipfeln bis zu knapp 2000 m Höhe als Doppelmassiv über $12 \mathrm{~km}$ in nord-nordwestlich - südsüdöstlicher Richtung. Hier kommen, anders als auf dem Xerovouni-Gebirge, sowohl St. rubicundus als auch St. clavatus vor, die dort, wo sich ihre Populationen überschneiden, miteinander hybridisieren.

\subsubsection{Nordtomaros}

Der nördliche Teil des Doppelmassives steigt, ähnlich wie der Xerovouni an den Hängen steil auf $1100 \mathrm{~m}$ an und bildet dort ein Plateau, das in südlicher Richtung kontinuierlich bis zu einer Höhe von 1800 m ansteigt. Der größte Teil dieses Plateaus wird von St. clavatus 
besiedelt. Ganz im Süden des Nordmassives befinden sich drei kleinere Gipfel (1766 m bis $1800 \mathrm{~m}$ ) auf denen St. rubicundus zu finden ist. Es deutet einiges darauf hin, dass diese Art auch im südlichen Teil des Osthanges, der jedoch schwer zu begehen ist, zu finden ist.

Im Bereich dieser drei Gipfel sind neben St. rubicundus auch St. clavatus zu finden. Des Weiteren findet man hier, nämlich etwas unterhalb des nördlichen und des südlichen der drei Gipfel, einzelne Tiere die sehr auffällige intermediäre Merkmale besitzen. Auch auf einer weiteren Erhöhnung, etwas östlich dieser drei Berge sind Tiere der Arten St. rubicundus und St. clavatus, aber auch einzelne Tiere mit offensichtlichen intermediären Merkmalen, vertreten.

\subsubsection{Südtomaros}

Auf dem Südmassiv kommt St. clavatus, ähnlich wie auf dem Nord-Tomaros, ebenfalls ab einer Höhe von 1300 m, vor. Hier besiedelt diese Art den Süd- und Westhang bis zu einer Höhe von 1800 m sowie ein sehr kleines Areal an der nördlichen Kante des Osthangs. Die Hänge sind ähnlich den Wiesen des Xerovouni sehr karg und steinig. Auf dem Nordhang des Südberges sowie dessen Gipfel findet man keine St. clavatus. Stattdessen sind hier Populationen von St. rubicundus zu finden. Diese erstrecken sich vom Nordhang hinunter bis zum Osthang des Südmassivs (Abb. 14). Das St. rubicundus-Habitat ist hier ähnlich dem Habitat des Valtou- und Mitsikeli-Massivs mit Gräsern der Gattung Festuca bewachsen. Einzig der Osthang des Berges weist etwas üppigere Vegetation mit vereinzelten Bäumen auf. Die Populationsdichte von St. rubicundus ist hier ähnlich hoch wie im Vernon-Gebirge.

An den Habitatgrenzen, an denen diese beiden Arten aneinander stoßen bilden sich Überschneidungszonen, an denen Tiere mit intermediärer Morphologie und Verhalten gefunden worden sind. Eine solche Hybridzone zieht sich auf einer Höhe von $1400 \mathrm{~m}$ bis 1800 vom Westhang des Südtomaros über einen westlichen kleineren Gipfel in südliche Richtung (Abb. 14). Von hier aus erstreckt sie sich über den Sattel in östlicher Richtung etwas unterhalb des Gipfels bis hin zum Osthang auf 1300 m. Des Weiteren wurden einzelne intermediäre Individuen auf 1750 m Höhe auf den nordöstlichen Teil des Südtomaros gefunden. Hierbei handelt es sich um ein sehr keines Gebiet in dem wenige St. clavatus und viele St. rubicundus vorkommen. 
Die auf dem Nord- und Südmassiv des Tomaros-Gebirges vorkommenden Heuschreckenarten St. rubicundus und St. clavatus sowie deren Übergangsformen (Hybriden) werden im Folgenden in morphologischer sowie ethologischer Hinsicht charakterisiert. Hierzu werden zum einen die Vorderflügel- sowie die Antennenmorphologie und zum anderen die Werbegesänge der Männchen herangezogen. Als Referenzgruppen dienen Individuen, von außerhalb des Tomaros-Gebirges vorkommenden Populationen von St. rubicundus und St. clavatus, die keinen Kontakt zu der jeweils anderen Art haben.
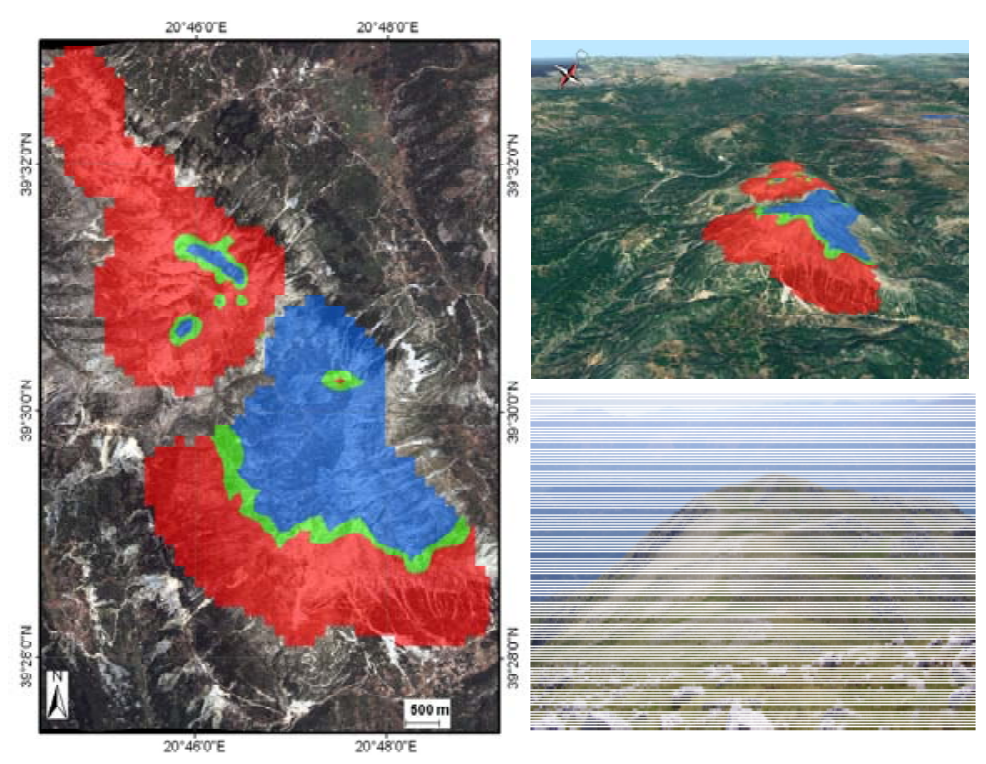

Abb. 14 Vermutete Populationsverteilung der Arten St. rubicundus (blaue Fläche), St. clavatus (rote Fläche) und Gebieten in denen intermediäre Tiere gefunden worden sind (grüne Fläche), auf dem Tomaros. Sattelitenbild (links), dreidimensionale Ansicht (oben-rechts) und Foto des Habitats (unten-links).

\subsubsection{Besonderheiten der Fundorte}

Bei der Beschreibung der Fundorte der beiden Arten St. rubicundus und St. clavatus fällt vor allem auf, dass vier der inselartigen Ausbreitungsgebiete in unmittelbarer Nähre zu einander zu finden sind. Dabei handelt es sich um den Mitsikeli, den Tomaros, den Xerovouni und das Valtou. Auf dem Mitisikeli und dem Valtou kommen St. rubicundus vor auf dem Xerovouni St. clavatus und der Tomaros beherbergt beide dieser Arten. Diese vier Berge sind durch tiefe Täler getrennt die große Lücken von mindestens $15 \mathrm{~km}$ zwischen den Habiten bedeuten. In Abb. 15 ist diese inselartige Aufteilung der Habitate nochmals durch ein Höhenmodell verdeutlicht worden. 


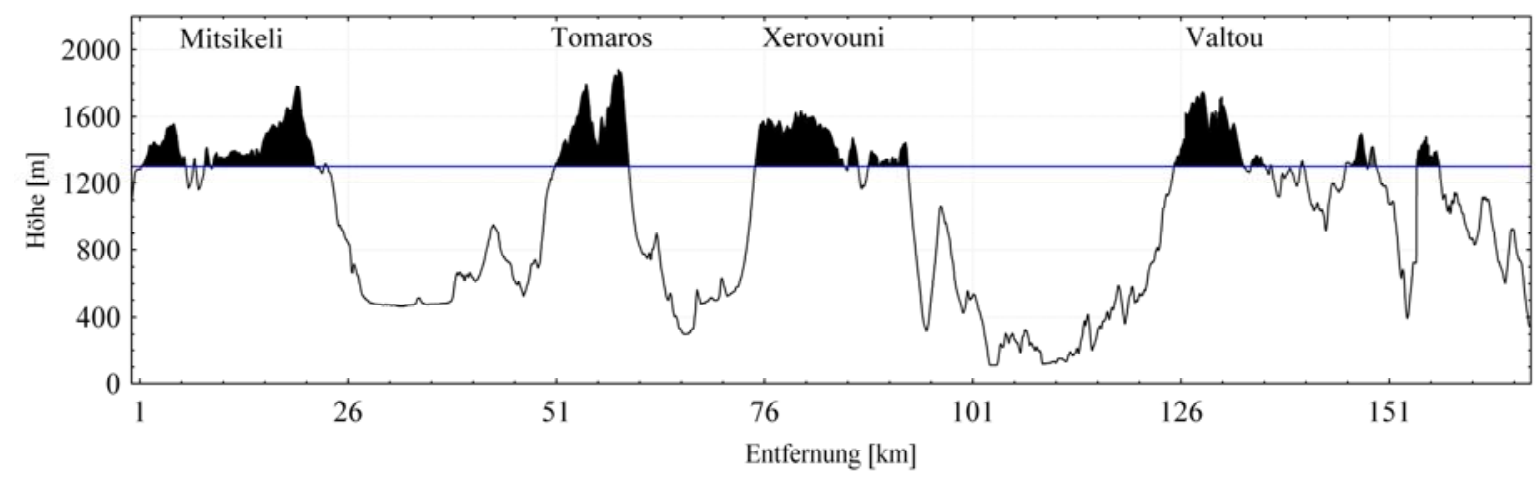

Abb. 15 Höhenmodell ausgesuchter Massive rund um die Stadt Ioannina. Die blaue Linie ist die untere Habitatsgrenze der beiden Arten bei 1300m. Die schwarzen Flächen verdeutlichten die Habitate der hier untersuchten Heuschreckenarten.

\subsection{Sammeln und Haltung der Versuchstiere}

Alle Versuchstiere wurden entweder im Freiland in den Jahren 2005-2009 gefangen, oder im Labor aufgezogen. Die Tiere wurden nach Art und Geschlecht getrennt in kleinen Kunststoffkäfigen (10-15 Tiere) oder in größeren Sammelkäfigen (bis zu 50 Tiere) gehalten. Als Futterpflanzen dienten ausschließlich Gräser der Gattung Festuca, die nach ca. 2 bis 3 Tagen ausgetauscht wurden. Im Freiland wurde jeder Fundort der Individuen mit einen GPS-Punkt markiert und jedes Tier, das in einem Radius von $20 \mathrm{~m}$ gefangen wurde, diesem zugeordnet.

Für Laboraufzuchten wurden zum größten Teil Nachkommen von im Freiland gefangenen Individuen verwendet. Um Nachkommen im Labor ziehen zu können, wurden die von den Weibchen in Vermiculite (Deutsche Vermiculite Dämmstoff $\mathrm{GmbH}$ ) abgelegten Eipakete in Petrischalen gegeben und im Kühlschrank bei ca. $7{ }^{\circ} \mathrm{C}$ für drei bis vier Monate verwahrt. Im Anschluss schlüpften die Larven nach ca. 10 bis 14 Tage im Wärmeschrank bei ca. $25{ }^{\circ} \mathrm{C}$. Die Heuschrecken wurden bei $25^{\circ} \mathrm{C}$ bis $30{ }^{\circ} \mathrm{C}$ mit einer Nachtabsenkung von um $10^{\circ} \mathrm{C}$ im $12 \mathrm{~h} \mathrm{Tag/Nacht-Rhythmus} \mathrm{aufgezogen.}$

\subsection{Morphometrische Vermessung der Vorderflügel}

\subsubsection{Aufnahme der Flügel und Setzen von Messpunkten}

Zur Charakterisierung von Individuen der beiden Arten St. clavatus und St. rubicundus sowie deren Zwischenformen wurde die Methode der flügelmorphometrischen Vermes- 
sung angewandt. In der Zoologie werden derartige Vermessungen mit Hilfe von anatomischen Messpunkten (Landmarks) unter anderem bei Fischen (Hjelm at al 2001, Barluenga et al. 2006; Clabaut et al. 2007) und Insekten (Pretorius 2005, Bischoff 2009, Francuski 2009) angewandt. Aber auch in der Anthropologie bei der Untersuchung von Skelettteilen werden solche morphometrischen Vermessungen durchgeführt (Brunner et. al. 2001, Penin et al. 2002, Frost et al. 2003). Im Zuge der vorliegenden Arbeit werden zum ersten Mal Vorderflügel von Heuschrecken mit dieser Methode vermessen.

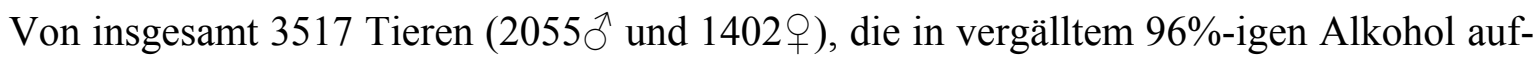
bewahrt wurden, wurden die Vorderflügel morphometrisch vermessen. Hierfür wurde in der Regel der rechte Vorderflügel an der Basis mit einer Präzisionsschere abgeschnitten. Falls dieser zu große Beschädigungen aufwies, wurde der linke Vorderflügel des Tieres, sofern unbeschädigt, zur Vermessung genutzt.

Die abgetrennten Vorderflügel wurden mittig zwischen zwei Deckgläschen (24x60mm Roth) mit etwas Glycerin (Firma), fixiert und mit einem Scanner (Epson PERFECTION 4490 Photo) im Durchlicht mit hoher Auflösung (4800 dpi; 3684 x 1644 Pixel) von der Oberseite her abgescannt. Die linken Vorderflügel wurden horizontal gespiegelt, sodass alle Flügel die gleiche Ausrichtung hatten. Dies geschah deshalb, weil der zu untersuchende Körper stets von der gleichen Seite aufgenommen werden sollte, um eine vergleichbare Merkmalskonfiguration zu gewährleisten (Auffray et al. 1999). Die Abspeicherung erfolgte im JPEG-Format.

Mit dem Programm TPSdig2 (Rohlf 2004) wurden an 13 charakteristischen Stellen (Abb. 16), anatomische Messpunkte („Landmarks“) gesetzt (LM 1 bis LM 13) und deren X- und y- Koordinaten bestimmt. Als Merkmale für die Festlegung der Landmarken, kommen bestimmte Stellen auf den Flügeln, etwa Verzweigungen der Adern, in Frage. Um jedoch ein solches Merkmal für biometrische Systeme nutzen zu können, müssen folgende Voraussetzungen erfüllt werden: Es muss sich zum einen um ein eindeutig zu erkennendes Merkmal handeln, z. B. die Aufgabelung von Flügeladern. Außerdem müssen solche Flügelmerkmale universell, also bei allen Individuen vorkommen. Sie dürfen sich nicht mit zunehmendem Alter der Individuen verändern. Morphometrische Merkmale sollten ferner eindeutig messbar sein, d. h. die Landmarks sollten nicht zu nah beieinander liegen oder eine zu große innerartliche Varianz aufweisen. 
Die Messpunkte wurden bei allen Individuen an den gleichen Stellen gesetzt; Abb. 16 illustriert dies am rechten Vorderflügel von St. clavatus. Von den anatomischen Messpunkten (Landmarks LM) befinden sich LM1 und LM2 an den Aufspaltungen der Costa, Subcosta und Media. Der Messpunkt LM9 wurde am posterioren Zusammenschluss der beiden Analadern gesetzt. Im Bereich des Stigmas befinden sich sechs Landmarks, von denen LM 10 bis LM13 das Stigma selbst umrahmen. Hierbei befinden sich LM10 und LM13 auf der Media und LM11 und LM12 auf dem Radius. Die Flügelader Radius spaltet sich zweimal auf. Bei der ersten Aufspaltung wird LM4 gesetzt. LM3 wurde an der Stelle definiert, an der die Cubitusader anterior abknickt. Landmarks, die jeweils das distale Ende einer Flügelader markieren, sind LM5 (Ende des Radius), LM6 (Ende der Media), LM7 (Ende des Cubitus) und LM 8 (Ende der Analis).

Die Koordinaten der 13 „Landmarks“ jedes Individuums wurden nach Normierung (siehe unter Kapitel 2.4.2) einer Hauptkomponentenanalyse (Kapitel 2.4.3) unterzogen. Auf diese folgte dann eine kanonische Varianzanalyse (Kapitel 2.4.4) mit der die Untersuchungen auf Populations- und Individuumsebene durchgeführt werden können. Die Programme, die für diese Untersuchung nötig sind, stammen aus dem frei verfügbaren Softwarepaket IMP (Sheets 2000).

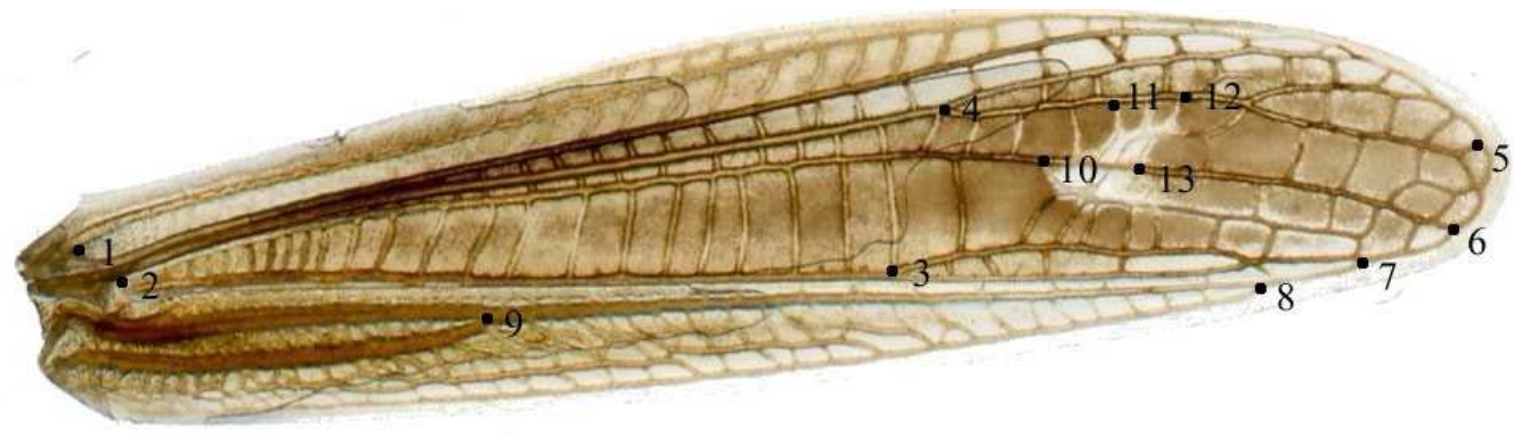

Abb. 16 Anatomische Messpunkte („Landmarks“) auf dem rechten Vorderflügel von St. clavatus.

Es folgen nun mehrere mathematische Rechenschritte, die nötig sind, um Populationsvergleiche und später Vergleiche auf Individuenebene durchführen zu können. Erstes Ziel ist es, Artunterschiede zwischen St. clavatus und St. rubicundus zu finden. Auf dieser Grundlage können dann später mögliche Hybriden mit den beiden Arten verglichen und exakt eingeordnet werden. 


\subsubsection{Normierung (Prokrustes-Analyse)}

Nachdem die Flügel an charakteristischen Stellen digital markiert wurden, folgt ein Normierungsschritt, um den Einfluss von inner- und außerartlichen Größenunterschieden zu minimieren. Im Zuge dessen wurden die vermessenen Vorderflügel der Individuen zusammengefasst und mit der Software CoordGen6 (Sheets 2000) durch eine ProcrustesAnalyse (Rohlf and Slice, 1990) normiert. Die Procrustes-Analyse stellt ein wichtiges Instrument im Zusammenhang mit multidimensionaler Skalierung dar (Gower 2001, Schönemann und Caroll 1970). Anwendung findet die Prokrustes-Analyse unter anderem bei geometrischen Figuren in der Natur, bei denen ihre Gestalt im Allgemeinen durch Translation, Rotation und Dilatation unverändert bleiben (Rohlf und Slice, 1990). Bei der Skalierung werden also die geometrischen Verhältnisse zwischen den Punkten beibehalten und einzig die Eigenschaften durch Translation, Rotation und Dilatation verändert (Dryden and Mardia 1998, Goodall 1991). Dadurch spielen innerartliche Größenunterschiede und verschiedene Ausrichtungen beim Abscannen keine Rolle mehr und gehen nicht in die Berechnung ein. Die zuvor gesetzten Pixelkoordinaten werden durch dieses Verfahren in sogenannte Booksteinkoordinaten (nach Bookstein [1991]; normierte Pixelkoordinaten) umgewandelt. Auf Grundlage dieses Normierungsverfahrens wird einzig auf die Gestalt des Vorderflügels, also auf das Verhältnis der Landmarken untereinander, Bezug genommen. Die so gewonnenen Daten werden nun mit den Programmen PCAgen6 (Hauptkomponentenanalyse; Kapitel 2.4.3) und CVAgen6 (kanonische Varianzanalyse; Kapitel 2.4.4) genauer untersucht.

\subsubsection{Hauptkomponentenanalyse}

Auf die zuvor beschriebenen Normierungsschritte folgt die eigentliche Analyse der morphometrischen Struktur der Vorderflügel durch die Hauptkomponentenanalyse. Die Hauptkomponentenanalyse dient der Untersuchung der Gesamtvarianz der Datenmenge. Es wird untersucht, ob sich die Merkmale der Individuen stark genug unterscheiden, um detaillierte morphometrische Berechnungen sinnvoll durchführen zu können. Zunächst wurden Vertreter der beiden Arten miteinander verglichen um Artunterschiede festzustellen und so mögliche Hybride einzuordnen. Bei dieser Arbeit wird die Hauptkomponentenanalyse mit den Programmen PCAgen6 (Sheets 2000) oder STATISTICA (Statsoft) durchgeführt und graphisch dargestellt. 
Im Detail dient die Hauptkomponentenanalyse dazu, umfangreiche Datensätze zu interpretieren, indem Komponenten, in diesem Fall morphologische Marker, bestimmt werden, die den größten bzw. kleinsten Einfluss auf den Datensatz, also auf die morphologischen Unterschiede zwischen Individuen, haben. Dies wird erreicht, indem eine Vielzahl statistischer Variablen (Landmarks), durch eine geringere Zahl möglichst aussagekräftiger Linearkombinationen („Hauptkomponenten“) durch Umrechnung ersetzt wird (Bishop 1995). Mit dieser Methode kann bestimmt werden, welche Daten welchen Einfluss auf den gesamten Datensatz haben. Dies geschieht in Form von Hauptkomponenten. Somit kann gezeigt werden, wie variabel große Datensätze sind und wie diese unterschieden werden. Bei diesem Verfahren wird also geprüft, ob es Unterschiede im gesamten zu untersuchenden Datensatz, also vor allem bei den Vorderflügelkoordinaten, gibt. Mit Hilfe der ChiQuadrat-Statistik (Morrison 1967) wird dann geprüft, ob es signifikante $(p<0,05)$ Unterschiede zwischen den Hauptkomponenten gibt. Wenn es also signifikante Unterschiede zwischen mindestens zwei Hauptkomponentenachsen gibt, also morphologische Eigenschaften, die eine ähnliche Varianz aufweisen, ist die Varianz des Datensatzes groß genug um mögliche Artunterschiede zu vermuten. In diesem Fall, wird mit der kanonischen Varianzanalyse (CVA) weiter verfahren, um die Populationen miteinander zu vergleichen.

\subsubsection{Kanonische Varianzanalyse}

Die kanonische Varianzanalyse wird in erster Linie für die Berechnung von Datengruppen, in diesem Falle, Populationen oder Arten verwendet. Für die Untersuchung der Heuschreckenarten/Populationen wurde das Programm CVAgen6 aus dem IMP Softwarepaket verwendet. Wenn die vorangegangene Hauptkomponentenanalyse signifikante Unterschiede im Datensatz, also zwischen den Individuen ergeben hat, kann nun geprüft werden, wie stark sich die Populationen oder auch Arten unterscheiden. Dafür muss der Datensatz in Populationen bzw. Arten eingeteilt werden. Basis für diese Einteilung ist in erster Linie der Fundort des Individuums.

Die kanonische Varianzanalyse (Canonical Variate Analysis, CVA) ähnelt der Hauptkomponentenanalyse, untersucht aber den Zusammenhang zwischen mehreren unabhängigen die Variablen, die eine möglichst große Variabilität zwischen zwei oder mehreren Gruppen aufweisen (Nolte und Sheets 2005). Dadurch werden anders als bei der Hauptkomponentenanalyse, nicht Hauptkomponenten, sondern kanonische Varianzen errechnet. Mit diesem Verfahren ist es also möglich, die Varianz innerhalb und zwischen den Gruppen zu 
bestimmen (Abb. 17). Als Signifikanztest dient hier die auf der CVA basierende MANOVA (multivariate analysis of variance; Kapitel 2.4.6), die prüft, wie viele CVA-Achsen sich signifikant $(p<0,05)$ unterscheiden. Es müssen sich mindestens zwei der Achsen signifikant unterscheiden, um die Berechnung weiter durchführen zu können. Dies ist der Fall, kann man von einem signifikanten Unterschied von mindestens zwei der Gruppen ausgehen. So können Aussagen getroffen werden, inwieweit sich die Individuen der gewählten Gruppen morphologisch voneinander unterscheiden.

Zusätzlich wird ein Gütemaß für den Achsenunterschied berechnet. Es handelt sich dabei um das inverse Gütemaß „Wilks Lambda“. Je größer der Eigenwert (Varianz), umso kleiner ist Wilks Lambda. Ein niedriger Wilks Lambda-Wert bedeutet somit eine geringe Reststreuung und daher eine gute Trennung der Gruppen. Wenn Wilks Lambda nahe null bzw. eins ist, deutet dies auf eine gute bzw. schlechte Trennfähigkeit der Diskriminanzfunktion hin. Dies bedeutet, dass sich die gewählten Populationen oder Arten morphologisch stark oder weniger stark unterscheiden.

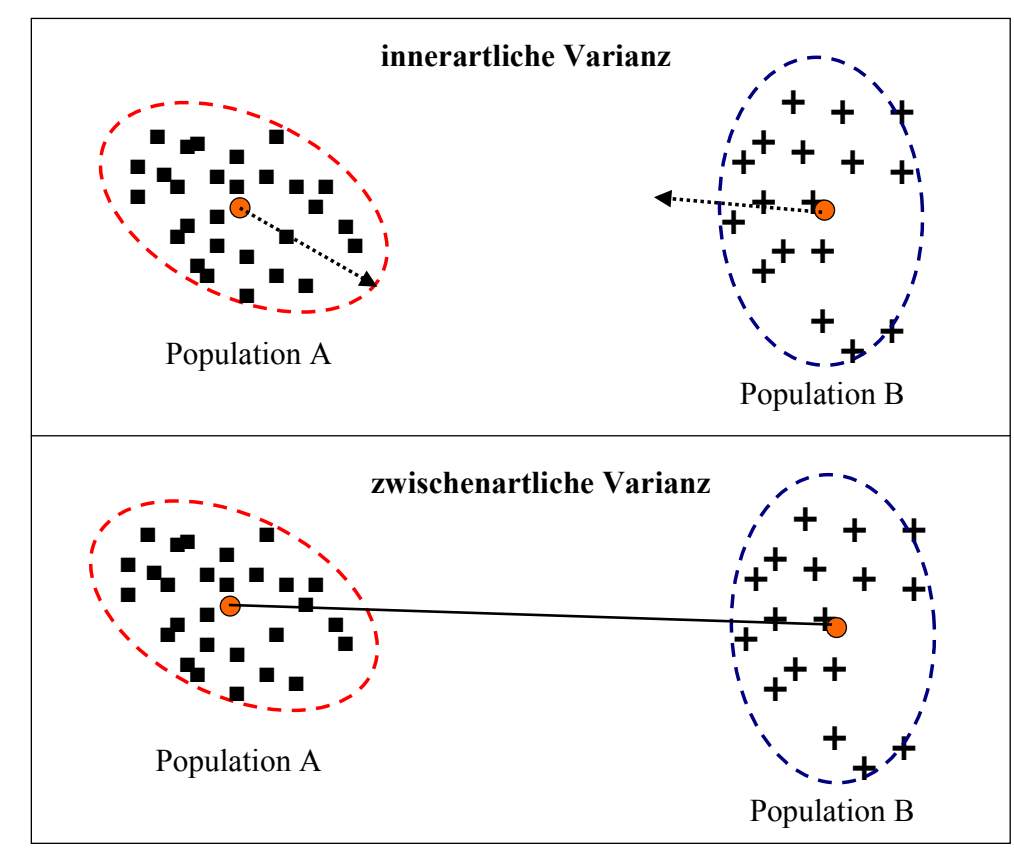

Abb. 17 Bestimmung von innerartlichen und zwischenartlichen Unterschieden durch kanonische Varianzanalyse. Schwarze Quadrate: Individuen der Population A, schwarze Kreuze: Individuen der Population B, roter Punkt: morphometrischer Mittelwert der Population. Gestrichelte Kreise: Varianz der Population. Pfeile: innerartliche Varianz. durchgezogene Linie: zwischenartliche Varianz.

Im nächsten Schritt muss nun geprüft werden, inwieweit die Gruppeneinteilung Einfluss auf die errechneten Daten hat, denn es ist möglich, dass Ausreißer in den Gruppen die Ergebnisse verzerren. Hierzu wurden die Ergebnisse der kanonischen Varianzanalyse dem Jackknife-Verfahren (Kapitel 2.4.5) unterzogen. 


\subsubsection{Jackknife-Verfahren}

Es kann sein, dass nur sehr wenige Individuen mit einer großen Variabilität zu den errechneten Unterschieden beitragen. Um diese Art von Fehlern auszuschließen, wurde ein Resampling-Verfahren (sinngemäß: Wiederholungs-Stichprobe) angewandt. Dabei handelt es sich um das sogenannte Jackknife-Verfahren. Dieses Verfahren, ein Spezialfall des Bootstrapping (Efron 1979), dient dazu, den zufälligen Fehler einer Schätzmethode und somit eine mögliche Verzerrung der Ergebnisse zu erkennen (Quenouille 1956). Bei den Daten der Stichprobe wird dazu jeweils ein Datensatz weggelassen und der Schätzwert für den reduzierten Datensatz berechnet. Dieser Test wurde mit dem Programm CVAgen6 durchgeführt, indem der Test mit 1000 Wiederholungen und einem Anteil weggelassener Werte einmal von $10 \%$ und einmal von $50 \%$ durchgeführt wurde. Wenn bei einem Ausschluss von 10\% mindestens noch $90 \%$ und bei einem Ausschluss von 50\% mindestens $80 \%$ der Daten richtig und signifikant waren, wurde die Gruppeneinteilung als richtig definiert. Das Jackknife-Verfahren ist von großer Bedeutung, da die Einteilung von Gruppen auf Basis der Fundorte und des Geschlechts der Tiere immer einer gewissen Willkür unterliegt. Wenn die Zuordnung dem Jackknife-Verfahren standgehalten hat, kann ein weiterer Signifikanztest, in diesem Falle die MANOVA, klären, ob der Unterschied zwischen den verwendeten Populationen eindeutig ist.

\subsubsection{MANOVA - Multivariante Varianzanalyse}

Bei der morphometrischen Untersuchung der Vorderflügel werden für jedes Tier 13 Messpunkte verwendet, die in die Untersuchung eingehen. Aufgrund dieser Vielzahl von Variablen wird für den Signifikanztest eine multivariante Analyse verwendet, die sogenannte MANOVA (multivariate analysis of variance). Sie trennt die gesamte Varianz der gemessenen Daten in die systematische Varianz und die unsystematische Varianz. Bei der systematischen Varianz handelt es sich um die Varianz, die auf experimentelle Veränderung zurückzuführen ist. Die unsystematische Varianz ist die, die aufgrund von individuellen Unterschieden, Messfehlern etc. zustande gekommen ist. Die Teststatistik gibt deren Verhältnis an, und damit die Wahrscheinlichkeit, dass die experimentelle Manipulation erfolgreich war, d. h. dass (bei einem Signifikanzniveau von 0,05) mit 95\%iger Wahrscheinlichkeit davon ausgegangen werden kann, dass gemessene Unterschiede in den Mittelwerten 
zwischen Bedingungen bzw. Gruppen tatsächlich vorhanden sind und nicht durch Zufall entstanden sind (Bray und Maxwell (1985).

Dieses Verfahren wird angewandt, um zunächst einmal mögliche morphologische Unterschiede zwischen den Vorderflügeln der Arten St. clavatus und St. rubicundus zu untersuchen. Wenn signifikante Unterschiede in der Vorderflügelmorphometrie zwischen den beiden Arten gefunden worden sind, werden alle anderen Individuen mit den beiden Arten verglichen. Dies geschieht, indem die Individuen von Gebieten, in denen Hybride zwischen den beiden Arten vermutet werden, als „unbekannt“ definiert und dann mit den beiden Arten verglichen werden. So kann bestimmt werden, ob ein Individuum der einen oder anderen Art zugeordnet werden kann, oder sich morphologisch zwischen den beiden Arten befindet.

Mit der zuvor verwendeten Hauptkomponentenanalyse (PCA) und der kanonischen Varianzanalyse (CVA) kann zum einen untersucht werden, an welchen Merkmalen sich die Vorderflügel des Datensatzes unterscheiden (Hauptkomponentenanalyse) und zum anderen, ob es signifikante Unterschiede zwischen den Gruppen gibt (kanonische Varianzanalyse). Beide Verfahren können aber nicht bestimmen, wie sich die einzelnen Individuen in diesem System verhalten, oder wie groß die Unterschiede einzelner Individuen zur eigenen bzw. zu anderen Gruppen sind. Diese innerartliche und zwischenartlichte Distanz jedes Individuums kann mit der Mahalanobis-Distanz (Kapitel 2.4.7) berechnet werden.

\subsubsection{Mahalanobis-Distanz}

Mit dieser Methode soll auf Basis der Daten aus der kanonischen Varianzanalyse bestimmt werden, wie groß der morphologische Unterschied der einzelnen Individuen zu der einen bzw. der anderen Art ist.

Bei der Mahalanobis-Distanz (Mahalanobis 1936) handelt es sich um ein Distanzmaß zwischen mehreren Punkten in einem mehrdimensionalen Vektorraum. Daher wird dieses Verfahren in der Statistik speziell im Zusammenhang mit multivarianten Verfahren, hierzu gehören auch die Hauptkomponentenanalyse (Principal Component Analysis [PCA]) und die kanonische Varianzanalyse (Canonical Variate Analysis [CVA]), verwendet. Basis für die Distanzberechnung ist die mehrdimensionale Datenmatrix der kanonischen Varianzanalyse. Jeder Punkt dieser Matrix beschreibt die Morphometrie eines Individuums und 
wird als mehrdimensionaler Spaltenvektor dargestellt. Es gehen also bei der MahalanobisDistanz-Berechnung alle Werte der CVA-Achsen jedes Punktes in die Berechnung mit ein. Somit wird im Detail die Mahalanobis-Distanz jedes Punktes (Individuums) der kanonischen Varianzanalyse zu dem Zentroid der jeweiligen Gruppe (Population) berechnet (Cornuet et al. 1999). Der Zentroid ist ein „Mittelpunkt“ im mehrdimensionalen Raum der die Mittelwerte aller unabhängigen Variablen jeder Gruppe repräsentiert (Abb. 18). Bildlich gesehen, ist der Zentroid das „durchschnittliche“ Individuum einer Population und repräsentiert diese somit. Auf diese Weise kann von jedem Individuum die innerartliche sowie die zwischenartliche Distanz bestimmt werden. Eine genaue Einordnung jedes Individuums ist somit möglich.

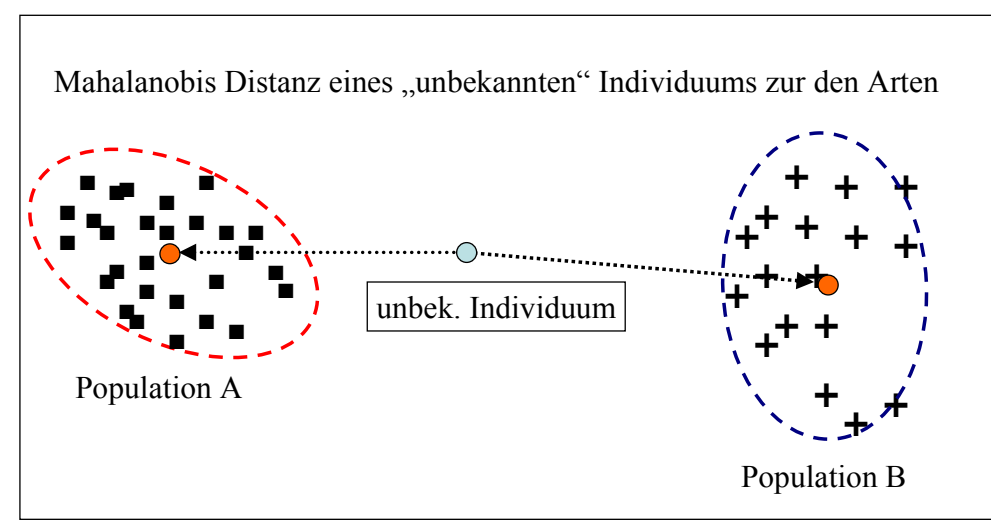

Abb. 18 Schema zur Bestimmung der Mahalanobis-Distanz. rote Punkte: morphometrischer Mittelwert der Population. Schwarze Quadrate: Individuen der Population A, schwarze Kreuze: Individuen der Population B gestrichelte Kreise: Varianz der Population. Pfeile: innerartliche Varianz. Line: zwischenartliche Varianz.

\subsubsection{Mahalanobis-Distanz-Differenz, Merkmalsanteile und Hybridindex}

Vor allem bei der Einordnung von unbekannten oder intermediären Individuen ist die Mahalanobis-Distanz von großer Bedeutung (Dillon und Manzi 1989). Die auf der kanonischen Varianzanalyse basierende Mahalanobis-Distanz bezeichnet für jedes Individuum die Distanz und somit die Varianz zwischen den definierten Gruppen (bzw. Arten). Bildet man die Differenz (Mahalanobis-Distanz-Differenz) aus beiden Werten, kann man die Individuen exakt einordnen. Umso kleiner die Werte sind, desto ähnlicher ist somit das betreffende Individuum beispielsweise der Population A; umso größer die Werte sind, umso ähnlicher der Population B.

Nun wird bestimmt, welches der Individuen der Population A bzw. B der Population B bzw. A am ähnlichsten ist. Dabei muss ausgeschlossen werden, dass sich die Merkmale der 
Populationen überschneiden oder große innerartliche Varianzunterschiede aufweisen. Ist dies nicht der Fall, kann die Mahalanobis-Distanz-Differenz zwischen dem Individuum der Population A, das der Population B am ähnlichsten ist, zum Individuum der Population B das der Population A am ähnlichsten ist, gemessen und in zehn gleich große Abschnitte unterteilt werden. Alle Tiere, die außerhalb dieser Einteilung zu finden sind, sind den jeweiligen Populationen A oder B zuzuordnen und bekommen den Hybridindex 1 für Population A und Index 12 für Population B. Zusätzlich hierzu wurde ebenfalls der prozentuale Anteil an St. rubicundus-Merkmalen ermittelt. Hierzu wurden den Individuen, die sich innerhalb der Varianz der Art St. rubicundus befanden, ein St. rubicundus-Merkmalsanteil von $100 \%$ zugeschrieben und Individuen, die Merkmale innerhalb der Varianz von St. clavatus aufweisen, ein St. rubicundus-Merkmalsanteil von 0\%. Intermediäre Individuen sind dementsprechend durch St. rubicundus-Merkmalsanteile von weniger als $100 \%$ und mehr als $0 \%$ gekennzeichnet (Abb. 19).

Alle Individuen die eine Mahalanobis-Distanz-Differenz besitzen, die zwischen den Populationen liegt, werden den Hybridindices 2 bis 11 zugeordnet. Somit erhalten Individuen, die der Population A, d. h. St. clavatus ähnlich sind einen Hybridindex von 2 bis 6 und jene, welche größere Gemeinsamkeiten zur Population B, d. h. St. rubicundus, aufweisen, einen Index von 7 bis 11.

HYBRIDINDEX

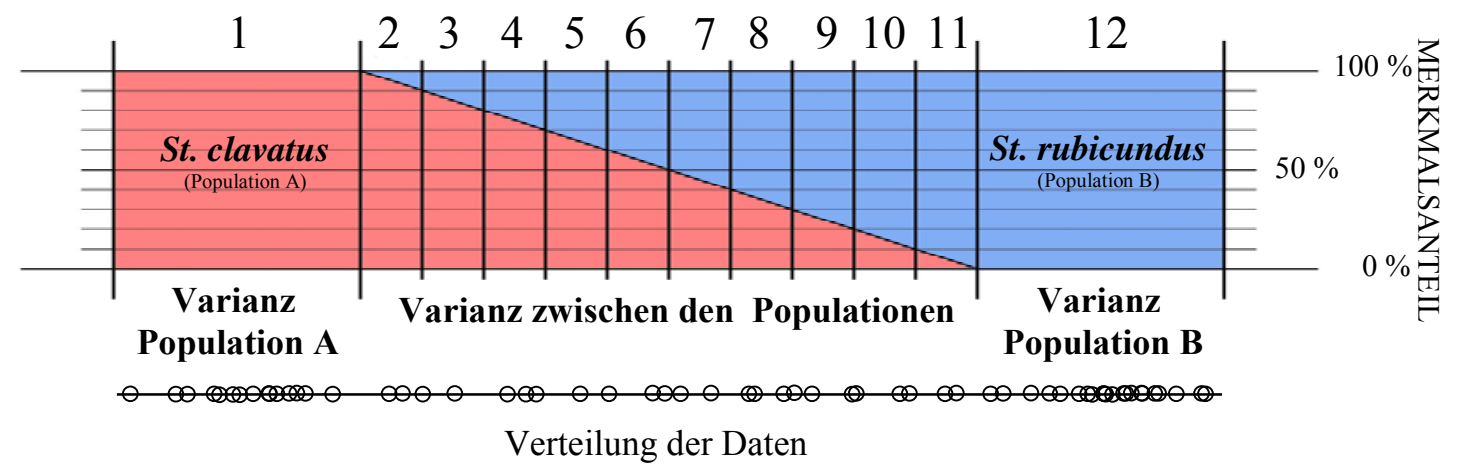

Abb. 19 Schematische Einteilung der Hybridindices (von links nach rechts) und der Merkmalsanteile (von unten nach oben) auf Grundlage der Verteilung von Einzeldaten (unten: Linie mit Punkten) der Flügelmerkmale. 


\subsubsection{Bestimmung des Flächeninhalts des Vorderflügels und der Länge des Femurs} - klassische Morphometrie

Zusätzlich zur morphometrischen Untersuchung der Vorderflügelform (siehe oben) wird der Flächeninhalt des Vorderflügels bestimmt, um zu klären, inwieweit sich morphometrische Veränderungen auf die Flügelgröße auswirken.

Die Vermessung wird mit dem Programm ImageJ (Rasband 1997-2005) durchgeführt. Hierzu wird ein Polygon deckungsgleich über den von oben aufgenommenen Vorderflügel gelegt, dessen basale Grenze an der Verzweigung der Flügeladern Costa, Subcosta und Media liegt (Abb. 20). Dabei wird die Basis des Vorderflügels, die durch das Abschneiden des Flügels oftmals beschädigt wird, ausgespart.

Der Flächeninhalt, des Flügels wird in Pixel gemessen und in Quadratmillimeter umgerechnet (Viloria et al. 2003; Weaver 2009). Die Vorderfügel werden, wie oben beschrieben, mit einer Auflösung von 4800 dpi aufgenommen. Alle Bilder haben eine Größe 19,5mm x 8,7 mm bzw. 3684 x 1644 Pixel. Somit entspricht ein Quadratmillimeter 35709 Pixel.

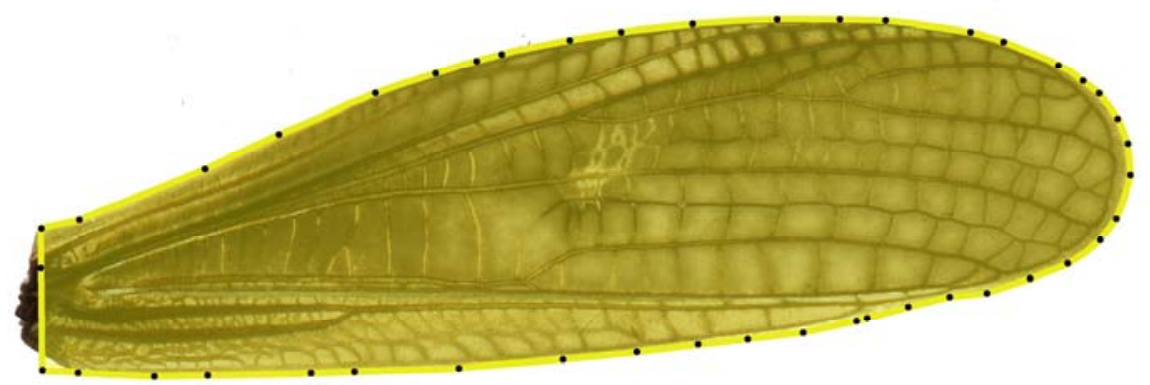

Abb. 20 Messbereich (gelb) für die Oberflächenberechnung des Vorderflügels (gelb)

Zusätzlich zur Bestimmung des Vorderflügelflächeninhalts wird die Länge des Femurs der Hinterbeine gemessen. Diese dient als Maß für die Körpergröße von Heuschrecken (Batcheler 1967, Wall und Begon 1987, Willott und Hassall 1998) zu der der Flächeninhalt des Flügels in Bezug gesetzt wird. Eine Vermessung erfolgte mit einer digitalen Schieblehre DMV-SL05 der Firma TOP CRAFT. Gemessen wird die Femurlänge in Millimeter, nach jedem zehnten Messvorgang wird die Schieblehre neu geeicht, um den Messfehler so gering wie möglich zu halten. Die Ergebnisse wurden mit der Statistik-Sotware STATISTI- 
CA von Statsoft ausgewertet. Die verschieden Populationen werden zusätzlich durch Signifikanztests wie ANOVA und als Pos-hoc Test dem Scheffé-Test miteinander verglichen.

\subsubsection{ANOVA - Univariante Analyse}

Die ANOVA (analysis of variance Searle 1971) ist anders als die MANOVA (multivariate Varianzanalyse) eine univariante Varianzanalyse. Mit einer einfaktoriellen ANOVA ist es möglich, unabhängige Stichproben auf eine Hypothese zu überprüfen, nach der die Mittelwerte einer Variablen in verschiedenen Fallgruppen in der Grundgesamtheit gleich groß sind. Mit der ANOVA können mehrere Mittelwerte miteinander verglichen werden. Es kann also geprüft werden, ob es Abweichungen zwischen den Mittelwerten der einzelnen Gruppen/Populationen gibt. Das Signifikanzniveau liegt hier, wie auch bei allen in dieser Arbeit verwendeten Signifikanztests, bei $p<0,05$. Ergeben sich nach Anwendung von ANOVA signifikante Unterschiede, kann mittels weiterer Tests, wie z. B. dem Scheffé-Test (Kapitel 2.4.10.1) der Unterschied zwischen definierten Gruppen errechnet werden.

\subsubsection{Scheffé-Test (Scheffé-Prozedur)}

Im Anschluss an die ANOVA wird der Scheffé-Test (signifikant bei $\mathrm{p}<0.05$ ) durchgeführt, um explizit Signifikanzunterschiede der Gruppen mit unterschiedlicher Individuenanzahl zu ermitteln. Der Scheffé-Test gehört zu den sogenannten Post-hoc-Verfahren. Es ist dies die allgemeine Sammelbezeichnung für Tests, die angewendet werden können, nachdem allgemeine Tests über mehrere Gruppen, wie z.B. ANOVA, eine Signifikanz ergeben haben. Sie werden auch als ungeplante Tests bezeichnet.

Der Scheffé-Test führt gemeinsame, paarweise Vergleiche für alle möglichen paarweisen Kombinationen der Mittelwerte der Gruppen/Populationen durch. Das Signifikanzniveau $(\mathrm{p}<0,05)$ des Scheffé-Tests ist so festgelegt, dass alle möglichen linearen Kombinationen von Gruppenmittelwerten getestet werden können und nicht nur paarweise Vergleiche verfügbar sind. Dies führt dazu, dass der Test oftmals konservativer ist als andere Post-hocVerfahren und somit für eine Signifikanz eine größere Differenz der Mittelwerte erforderlich ist. 


\subsection{Morphometrische Vermessung der Antennen}

Ein weiteres wichtiges Unterscheidungsmerkmal zwischen St. clavatus und St. rubicundus ist die Gestalt der Antennen. Zur quantitativen Erfassung der Antennenmorphologie wurden von 1117 (740 ठ̊; 377 ㅇ) Individuen die Antennen vermessen. Hierzu wurde von jedem Individuum eine intakte Antenne an der Basis zwischen Scapellus und Pedicellus mit einer Präzisionsschere abgeschnitten und ähnlich wie bei der morphometrischen Vermessung der Vorderflügel, zwischen zwei Deckgläschen (ROTH) mit Hilfe von etwas Glycerin (Merck) fixiert. Das Glycerin wurde lediglich in sehr kleinen Mengen links und rechts von den Antennen verteilt und kam mit dem Objekt selbst nicht Berührung. Es musste darauf geachtet werden, dass die Antennen flach auf den dünnen Glasscheiben liegen. Im folgenden Schritt wurde die fixierte Antenne mit einer Auflösung von 4800 dpi mit einen Flachbettscanner (siehe Kapitel 2.4.1.) aufgenommen und die Datei im JPEG-Format gespeichert. Die anschließende morphometrische Vermessung wurde mit dem Computerprogramm ImageJ durchgeführt. Zum einen wurde die Breite des ersten Antennensegmentes (Bereich A), zum anderen die breiteste Stelle im Bereich der letzten Antennensegmente (Bereich B) gemessen. Die Werte wurden in Pixel bestimmt und später in Millimeter umgerechnet $(1 \mathrm{~mm}=189$ Pixel). Das Verhältnis der Antennenbreite im Bereich B zu jener in Bereich A wird als antennaler morphometrischer Wert bezeichnet und für die Einordnung der Individuen herangezogen (Abb. 21). Die mathematischen Berechnungen wurden mit dem Tabellenkalkulationsprogramm EXCEL und STATISTICA (Statsoft - Version 8) durchgeführt.

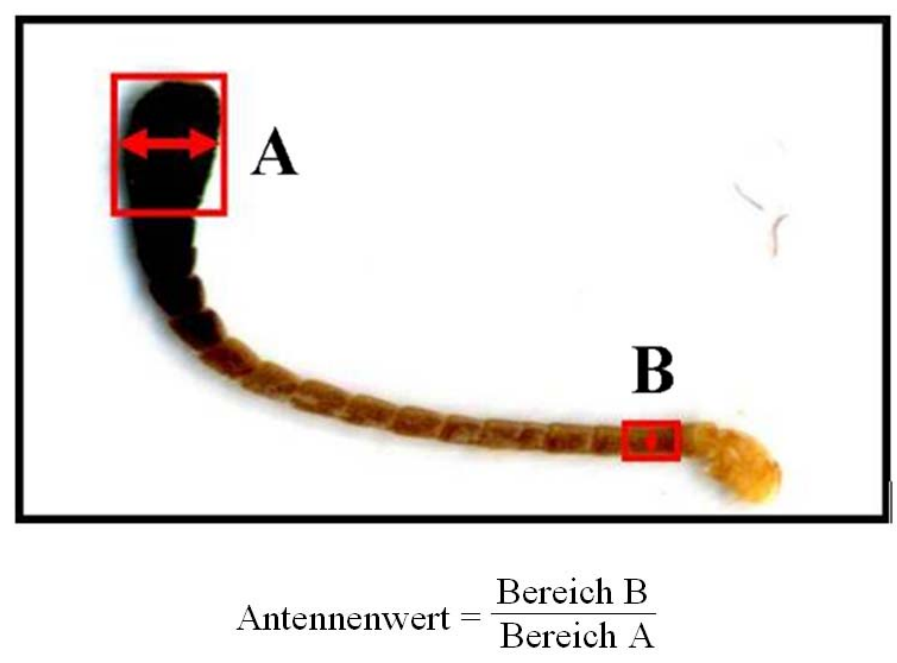

Abb. 21 Schema zur Berechnung des Antennenmerkmals. rote Pfeile: Messbereich. 
In gleicher Weise wie bei der Flügelmorphologie werden auch hier für jedes Individuum ein Hybridindex und ein Merkmalsanteil berechnet. Alle Individuen, deren antennaler morphometrischer Wert innerhalb der Varianzen der Arten St. clavatus bzw. St. rubicundus liegt, erhalten einen Hybridindex von 1 (St. clavatus) bzw. einen Index von 12 (St. rubicundus). Individuen die morphologisch zwischen den beiden Arten liegen, sind entsprechend ihrer Nähe zu St. clavatus bzw. St. rubicundus durch Hybridindices von 2 bis 11 gekennzeichnet. (Abb. 22)

HYBRIDINDEX

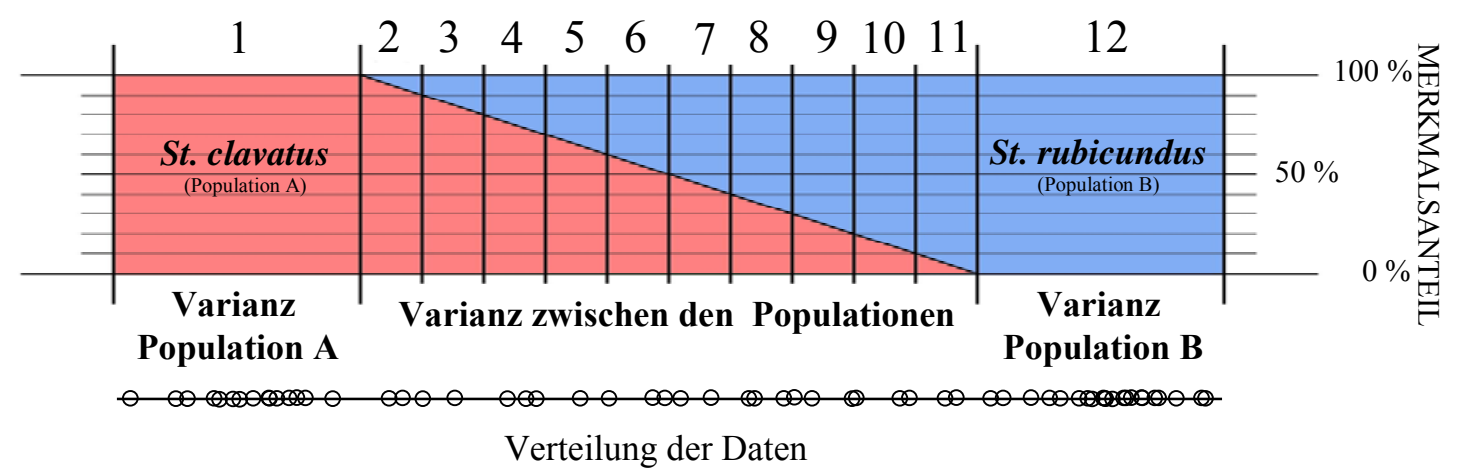

Abb. 22 Schematische Einteilung der Hybridindices (von links nach rechts) und der Merkmalsanteile (von unten nach oben) auf Grundlage der Verteilung von Einzeldaten (unten: Linie mit Punkten) der Antennenmerkmale.

Der Merkmalsanteil jedes Individuums wurde genau wie bei der morphometrischen Vermessung der Vorderflügel bestimmt. Das bedeutet also, dass ein Tier mit einem Merkmalsanteil von $100 \%$ innerhalb des Varianzbereichs von St. rubicundus liegt, während eines mit dem Merkmalsanteil von 0\% innerhalb des Varianzbereiches von St. clavatus zu finden ist. Intermediäre Individuen liegen dementsprechend dazwischen, d. h. ein Tier mit beispielsweise einem Merkmalsanteil von 35\% weist zu 65\% St. clavatus-Merkmale und zu 35\% St. rubicundus-Merkmale auf.

\subsection{Untersuchung des Heuschreckengesangs}

\subsubsection{Der Versuchsaufbau}

Zusätzlich zur morphometrischen Vermessung wurde der Balzgesang der Männchen aufgezeichnet und anhand von mehreren charakteristischen Merkmalen ausgewertet. Hierzu 
wurden die Stridulationsbewegungen des rechten und linken Hinterbeins mit je einer optoelektronischen Kamera (Helversen und Elsner 1977) registriert. Am distalen Femurende, also nahe dem Femur-Tibia-Gelenk, wurden dafür kleine retro-reflektierende Folien (Scotchlite-M3) angebracht, die einen Durchmesser von $2 \mathrm{~mm}$ hatten. Die Versuchstiere wurden über die Spiegeloptik der Kamera, die einen Lichtfleck von ca. 10cm Durchmesser erzeugte, angeleuchtet. Das von der Folie in die gleiche optische Achse reflektierte Licht fiel als Lichtpunkt auf eine planare, $3 \mathrm{~cm}$ lange, einachsige Photodiode und wurde in linear von der Position des Beins abhängige Spannungswerte umgesetzt.

Das Heuschreckenmännchen und das anzubalzende Weibchen saßen auf einer drehbaren, mit schwarzem Samt bezogenen Heizplatte. Zeitgleich zu den Stridulationsbewegungen wurde ebenfalls das erzeugte Tonsignal mit einem Mikrofon (Brüel und Kjaer 4133), das ca. $5 \mathrm{~cm}$ bis $10 \mathrm{~cm}$ über dem Männchen angebracht wurde, aufgezeichnet. Die Beinbewegungen sowie der Heuschreckengesang wurden digital mit dem Computerprogramm LabVIEW7 (National Instruments) abgespeichert, um schließlich mit dem Computerprogramm DIADEM ausgewertet zu werden.

\subsubsection{Auswertung des Werbegesangs}

Bei einer Temperatur zwischen $35^{\circ} \mathrm{C}$ und $41^{\circ} \mathrm{C}$ wurden die Gesänge von 219 Männchen der Arten St. rubicundus St. clavatus deren Vertreter auf dem Tomaros sowie von Nachkommen aus Laborkreuzungen aufgezeichnet. Die Abtastrate der Positionsdetektoren lag bei $40 \mathrm{kHz}$. Von jedem Tier wurden mindestens zwei komplette Balzgesänge, also Gesänge die aus mindestens drei verschiedenen Phasen bestehen, aufgezeichnet. Dabei wurde darauf geachtet, dass das Tier sich immer in der gleichen Position zu den beiden optoelektronischen Kameras und dem Mikrofon befand. Dies konnte durch die drehbare Heizplatte, auf dem das Tier während der Aufzeichnung saß, gewährleistet werden.

Es wurden mehrere Gesangsmerkmale der Arten St. clavatus und St. rubicundus untersucht und nur jene für die Analyse verwendet, die sich signifikant (ANOVA; $p<0,001$ ) zwischen den beiden Elternarten unterschieden. Die Gesangsanalyse bestand somit aus acht Parametern (Merkmale), die alle Gesangsphasen abdecken. Jedes Merkmal wird für jeden Gesang bei 10 bis 15 Ereignissen gemessen. Es wurden für die meisten Individuen mindestens zwei Gesänge detailliert untersucht und der Mittelwert aus den gemessenen Parametern für 
jedes Merkmal gebildet. Es wird im Kapitel 3.4 näher auf die einzelnen Merkmale noch eingegangen.

\subsubsection{Hybridindices und Merkmalsanteile der Gesangsmerkmale}

Wie bei der Untersuchung der morphologischen Daten werden auch bei der Analyse der Gesänge den Individuen jeweils ein Hybridindex sowie ein Merkmalsanteil zugeordnet.

Hierzu werden die Merkmale logarithmiert und einer Hauptkomponentenanalyse (Kapitel 2.4.3) unterzogen. Der Wert auf der ersten Hauptkomponentenachse (PC1) wird als Merkmal verwendet, da in der Regel auf dieser Achse die größte Varianz aller Werte zu finden ist. Diese Vorgehensweise ist in der Wissenschaft weit verbreitet und wird unter anderem bei verhaltensbiologischen Untersuchungen verwendet (Vedenina 2010). Die Einordnung erfolgt, wie bei der Morphologie, nach folgenden Gesichtspunkten: Individuen, die auf Grundlage des Wertes auf der Hauptkomponentenachse I (PC1) innerhalb der Varianz einer der beiden Elternarten liegen, werden dieser zugeordnet. Sie erhalten somit einen Hybridindex von 1 (St. clavatus) oder 12 (St. rubicundus) sowie einen Merkmalsanteil von 0\% (St. clavatus) bzw. 100\% (St. rubicundus). Zusätzlich werden die Individuen, die zwischen den beiden Arten liegen, aufgrund ihrer Stellung im Hauptkomponentensystem den Indices 2 bis 11 bzw. Merkmalsanteilen von größer als 0\% und kleiner als 100\% zugeordnet. Durch diese Einteilung sind Morphologie und Verhalten gut miteinander vergleichbar.

\subsection{GIS-basierte Untersuchung der Merkmalsverteilung}

\subsubsection{Geographische Informationssysteme (GIS)}

Mit geographischen Informationssystemen (GIS) können raumbezogene Daten digital erfasst und verarbeitet werden. Dies ist ein System das geographische (räumliche) Information (Daten) speichert, analysiert und visualisiert (DeMers 2000). Roger Tomlinson hatte 1984 das erste geographische Informationssystem, das Canada GIS erstellt. In der Evolutionsbiologie wurde GIS (geographische Informationssystem) erstmals von Kohlmann et al. (1988) bei der Untersuchung an chromosomalen Unterarten der australischen Heuschreckart Caledia capitva verwendet. 
In der vorliegenden Arbeit wird das geographische Informationssystem ArcGIS der Firma ESRI verwendet. Mit diesem Programm können die erhobenen Daten geographisch dargestellt werden, um so einen räumlichen Bezug der Merkmalsverteilung zu untersuchen. Grundlage für die Berechnung und Darstellung von geographisch basierten Daten ist das Koordinatensystem: WGS 1984 UTM Zone 34N. Die Höhendaten werden in einem Raster mit einer Zellgröße von 90m x 90m angezeigt und als Oberflächenmodell der Firma ESRI zur Verfügung gestellt. Da die Auflösung frei verfügbarer Satellitenbilder in ArcGIS für die exakte Kartierung der Heuschrecken angesichts der äußerst schmalen Hybridzone nicht ausreicht erscheint, wurde eine Aufnahme in Auftrag gegeben. Sie hat eine Auflösung von $1 \mathrm{~m} /$ Pixel und wurde am 24. Juli 2006 vorgenommen. Insgesamt erfasst die Satellitenaufnahme das gesamte Tomarosmassiv, so dass die jeweiligen Fundstellen genau erfasst werden können.

Es wurden insgesamt 156 GPS-Punkte im Abstand von mindestens 50m gesetzt und deren Höhe auf Basis des von ESRI zur Verfügung gestellten Höhenrasters bestimmt. An jedem dieser Punkte wurden 2 bis 110 Individuen (durchschnittlich 20 Individuen) gefangen und morphometrisch vermessen. Es wurden der prozentuale Merkmalsanteil und der prozentuale Anteil an Hybridindices der Individuen für jeden Fundort (GPS-Punkt) geographisch dargestellt. Dadurch kann man einen Überblick über die Verteilung von Merkmalen über ein bestimmtes Gebiet erhalten. Auf Grundlage durchschnittlicher Merkmalsanteile der Individuen jedes Fundortes können geographische Berechnungen wie die z.B. Interpolationen (Kapitel 2.7.2) dargestellt werden.

\subsubsection{Interpolation}

Nachdem die erhobenen Daten geographisch dargestellt wurden, können zum einen durch Interpolation (Kapitel 2.7.2) Lücken im Datennetz der Fundorte geschlossen und zum anderen kann die Position der Zentren der Hybridzone bestimmt werden. Abschließend kann durch geographische Distanzbestimmung mit dem GIS-Werkzeug „NEAR“ (Kapitel 2.7.3) die kürzeste Distanz der Datenpunkte zum Zentrum der Kontaktzone und anschließend der Verlauf der Merkmalskline (Kapitel 2.7.4) bestimmt werden.

Mit Interpolationswerkzeugen, die im ArcGIS-Softwarepaket $\mathrm{zu}$ finden sind, kann eine kontinuierliche Oberfläche aus stichprobenartig gemessenen Werten erstellt werden. Diese 40 
Oberfläche, die als Grundgerüst der Interpolation dient, besteht aus einem geographischen Raster, dessen Zellen eine Größe von 50x50 Meter haben. Die minimale Zellgröße ist durch den kleinsten Abstand (Minimum 50m), die zwei GPS-Punkte zueinander haben, definiert (Abb. 22). $\mathrm{Zu}$ den einfachsten, in der bei Untersuchungen von Hybridzonen (Swenson 2008) am häufigsten verwendeten Werkzeugen gehört die distanzbasierte Interpolation (Inverse Distance Weighted; IDW), die auch für diese Untersuchung angewendet wird.

Mit dieser werden, basierend auf dem Wert und dem Abstand nahe gelegener Punkte, Oberflächenwerte für die einzelnen Zellen geschätzt. Das heißt, dass durch Interpolation ein zusammenhängendes Raster entsteht, das aus vielen quadratischen Rasterzellen besteht. Jede dieser Rasterzellen (50mx50m) erhält durch Interpolationsverfahren einen Wert. Die einzelnen Zellen des Rasters bilden eine Oberfläche und ein zusammenhängendes Bild der Merkmalsverteilung.

Die interpolierten Werte für IDW-Oberflächen stellen einen Durchschnitt der Werte einer Reihe von nahe gelegenen Punkten dar, die so gewichtet werden, dass der Einfluss von nahe gelegenen Punkten größer ist, als der von weiter entfernten Punkten (Abb. 23). Es werden also die Werte der Rasterzellen in der Umgebung von Messpunkten geschätzt. Somit kann eine kontinuierliche Oberfläche entstehen, die der Verteilung von Merkmalen in einem Gebiet sehr nahe kommt.

Die Berechnung erfolgt durch die Formel:

$$
\hat{v}_{1}=\frac{\sum_{i=1}^{n} \frac{1}{d^{p}} v_{i}}{\sum_{i=1}^{n} \frac{1}{d^{p}}}
$$

Zur Formel: $\hat{v}$ ist der zu schätzender Wert, bei $v_{i}$ handelt es sich um den bekannten Wert, also um die zuvor ermittelten Messpunkte. Weiter beschreibt die Formel die exponenzierten (p) Distanzen $\left(d^{\mathrm{p}}{ }_{\mathrm{i}}, d^{\mathrm{p}}{ }_{\mathrm{n}}\right)$ der Datenpunkte (n) zum geschätzten Punkt (Ware et al. 1991). 
Durch das Exponenzieren der Distanz wird deren Einfluss zwischen den bekannten Datenpunkten gesteuert. Dabei gilt: Umso größer p, desto stärker ist der Einfluss der Distanz. In der vorliegenden Arbeit wurde ein Faktor von $\mathrm{p}=2$ benutzt (Jones und Searle 2003, Yanchukov et al. 2006). Dieser Wert gilt als Standard für derartige Berechnungen. Unter anderem wurden bei dieser Interpolation weitere Parameter für eine räumliche Begrenzung gewählt, somit gingen alle Rasterzellen in die Berechnung mit ein, die sich in einem Umkreis von bis zu 500 m um den jeweiligen Messwert befinden. Dieser Radius ist so gewählt, dass nur sehr wenige Rasterzellen außerhalb der Habitatsgrenzen (unterhalb von $1.300 \mathrm{~m}$ ) in die Interpolation mit einfließen. Es wurde nur dann eine Interpolation der Rasterzellen durchgeführt, wenn sich in diesem Umkreis mindestens zwei bekannte Messpunkte befinden. Bei den bekannten Messpunkten handelt es sich um die gemittelten, prozentualen Merkmalshäufigkeiten von St. rubicundus-Merkmalsausprägungen (Kapitel 2.4.8). Es werden insgesamt die drei Ausprägungen von Verhalten, Flügel- und Antennenmorphologie verwendet. Durch dieses Verfahren ist es nun möglich, das Zentrum der Hybridzone zu bestimmen und Merkmalsanteile in Gebieten ohne Messpunkte zu schätzen.
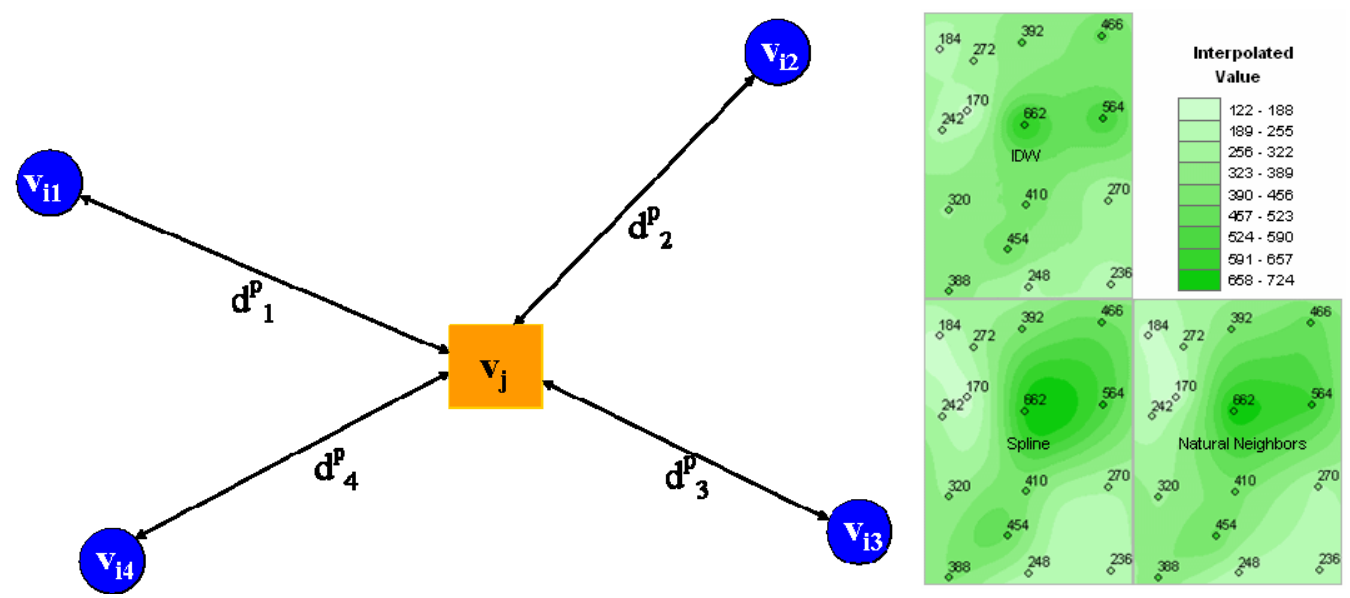

Abb. 23 Schema zur Interpolation von Rasterzellen auf Grundlage bekannter Messpunkte (links) Beispiel für eine interpolierte Rasteroberfläche (rechts) nach ESRI Hilfe (Mitchell 2005)

\subsubsection{Bestimmung des Zentrums der Hybridzone}

Als Zentrum der Hybridzone, wird jene Stelle bezeichnet, an der die beiden Arten aneinander stoßen und somit die Wahrscheinlichkeit der Hybridisierung am größten ist. Diese schmale Line ist im Bereich, an denen die interpolierten Zellen des Rasters mit interpolierten Merkmalsanteilen von $49 \%<\mathrm{p}<51 \%$ vorhanden sind, $\mathrm{zu}$ finden (Yanchukov et al. 2006). 
Nach der Bestimmung der Kontaktzone kann nun mit der „NEAR“ Funktion die genaue Position der Fundorte (GPS-Punkte) im Bezug zum Zentrum der Kontaktzone ermittelt werden (Abb. 24). Mit dem Werkzeug „NEAR“ wird vor allem die kürzeste Entfernung (in Metern) zur Kontaktzone, die eine Linie beschreibt, gemessen, aber auch der Winkel zu dieser bestimmt. Somit kann man klar bestimmen, auf welcher Seite der Kontaktzone sich die Datenpunkte befinden. Angegeben werden die Winkel von $-180^{\circ}$ bis $+180^{\circ}$, wobei $0^{\circ}$ Osten, $+90^{\circ}$ Norden, $180^{\circ}\left(-180^{\circ}\right)$ Westen und $-90^{\circ}$ Süden ist (Mitchell 2005). Bruchteile eines Grades werden hierbei nicht in Minuten und Sekunden, sondern als Dezimalwerte angegeben. Diese Werte beschreiben, in welchem Winkel die Kontaktzone zum Datenpunkt zu finden ist. Somit können die Datenpunkte exakt einer der beiden Seiten der Kontaktzone zugeordnet werden.

\begin{tabular}{|r|r|r|r|}
\hline & FeaturelD & NearDist & NearAngle \\
\hline & 0 & 56 & 18.394009 \\
\hline$\square$ & 1 & 122 & -31.848772 \\
\hline$\square$ & 195 & -2.41069 \\
\hline$\square$ & 48 & -35.72168 \\
\hline$\square$ & 3 & 105 & -13.856518 \\
\hline$\square$ & 4 & 177 & -10.703785 \\
\hline$\square$ & 5 & 75 & -23.185714 \\
\hline
\end{tabular}
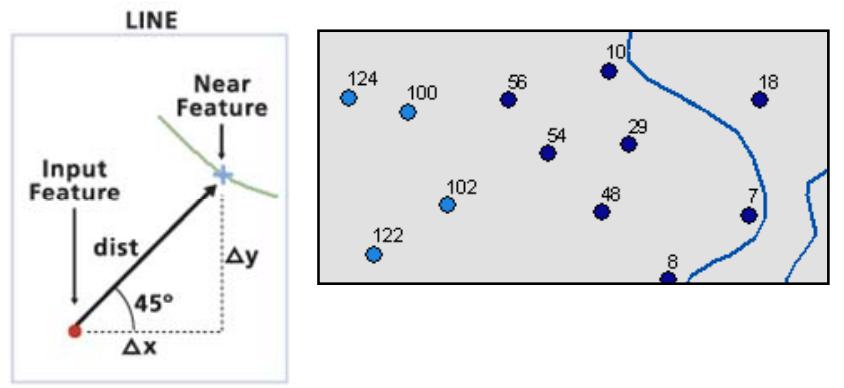

Abb. 24 Schema zur Bestimmung der kürzesten Distanz (NEAR) zur Kontaktzone. Tabelle rechts: Distanzund Winkelwerte, Mitte: Schema zur Bestimmung des Winkels und der Distanz zum Objekt (Kontaktzone), links: Beispiel einer Distanzbestimmung. Verändert nach ESRI Hilfe (Mitchell 2005).

\subsubsection{Kline}

Basis einer umfassenden Untersuchung von Hybridzonen sind vor allem die genaue Bestimmung des Zentrums der Hybridzonen sowie der Verlauf von Klinen.

Ein Kline ist die Merkmalsabstufung entlang eines geographischen Gradienten. Es hat meist die Form einer sigmoiden Kurve zwischen zwei Populationen entlang eines Transektes. Kline können dadurch eine ausgedehnte (graduale) oder eine enge (steile) Kurve beschreiben, dies hängt von der Überlebensrate der Hybriden und der damit verbundenen Rekombination von Merkmalen ab (Barton, 1983). Bei der Untersuchung der Kline ist also die räumliche Verteilung der Merkmale von großer Bedeutung. In vielen Arbeiten werden Transekte durch die Untersuchungsgebiete gelegt um mit Hilfe derer mögliche Merkmalsabstufungen zu untersuchen. In der vorliegenden Arbeit werden keine Transekte verwendet, sondern die kürzeste Distanz (,NEAR“) jedes Fundortes zur Kontaktzone, die durch 
Interpolation bestimmt worden ist, ermittelt. Durch Entfernungen mit positiven oder negativen Vorzeichen werden zusätzlich die Seiten der Kontaktzone definiert. Somit erhalten alle Fundorte der einen Seite eine kürzeste Distanz mit positiven und alle Fundorte der entgegen gesetzten Seite der Kontaktzone eine Distanz mit negativem Vorzeichen. Dies hat den Vorteil, dass in der zweidimensionalen Darstellung in der die kürzeste Distanz zur Kontaktzone gegen die durchschnittlichen Merkmalsanteile der Individuen an den einzelnen Fundorten aufgetragen wird, alle Fundorte mit einbezogen werden können.

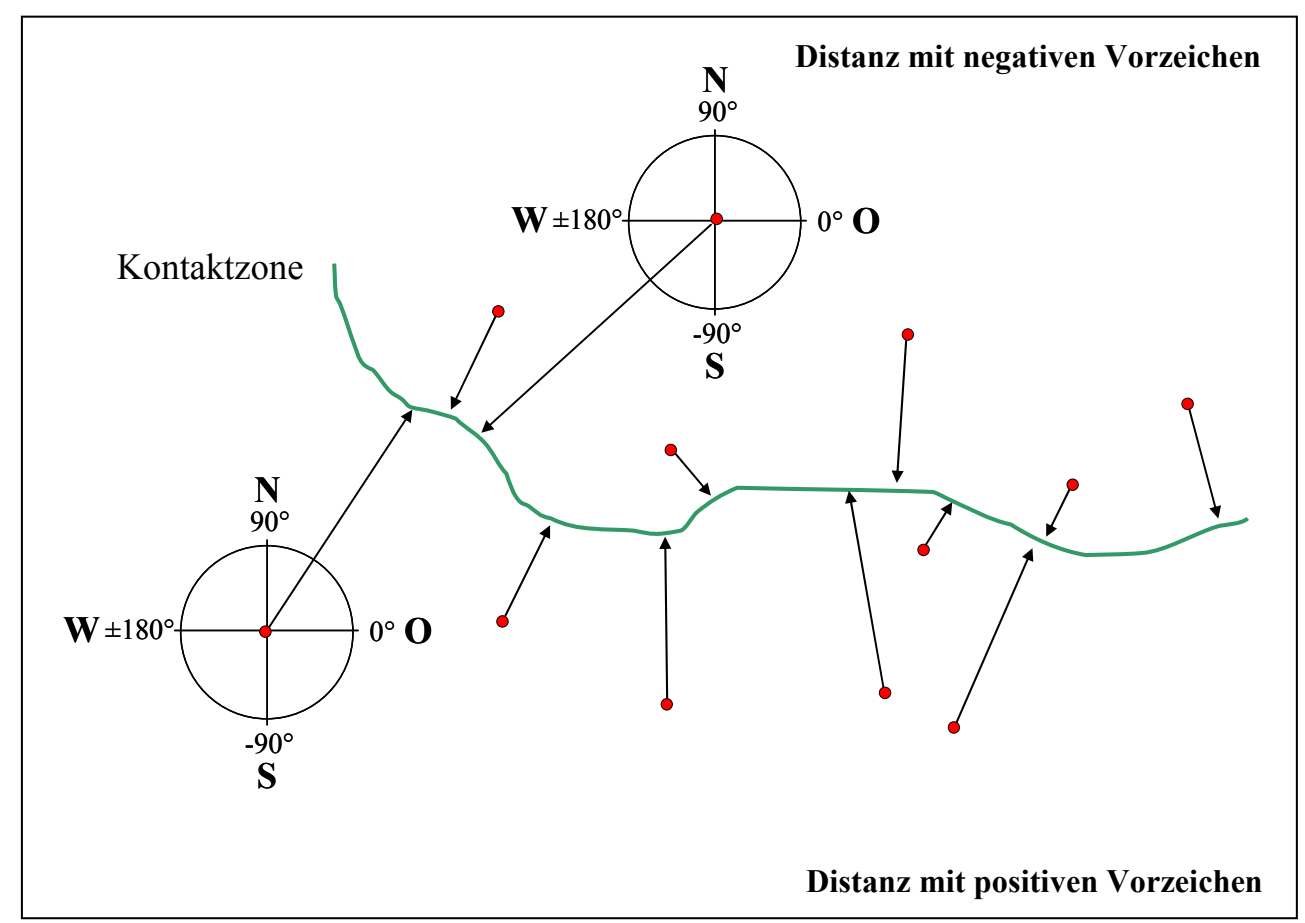

Abb. 25 Schema zur Bestimmung der kürzesten Distanz und des Winkels zur Kontaktzone (grüne Linie) von den jeweiligen Fundorten (rote Punkte).

Zum Vergleich der Kline wurde die von Barton und Hewitt (1985) und Kruuk (1997) vorgeschlagene Regressionsmethode verwendet. Sie besagt, dass wenn im Zuge der Regressionsanalyse die zu vergleichenden Größen signifikante Unterschiede zur quadratischen Größe der Regression aufweist, dass dann die Kline gegeneinander verschoben sind und somit ein unterschiedliches Zentrum haben. Bei signifikanten Unterschieden zur kubischen Größe, sind die Kline unterschiedlich groß oder weit. Diese Analyse wurde mit dem Programm STATISTICA durchgeführt. 


\subsection{Kreuzungsversuche}

Zusätzlich zu den Untersuchungen der Individuen im Feld oder deren Nachkommen, die im Labor gezogen wurden, sind zwischenartliche Kreuzungen durchgeführt worden. Diese dienen in erster Linie dazu, zu klären, wie sich Hybridisierungen im Feld über Generationen hin entwickeln können und inwieweit mögliche Evolutionsbarrieren wirken (Helversen und Helversen 1975a,b; Perdeck 1957, Saldamando et al. 2005).

Es wurden für diese Versuche hauptsächlich Individuen der allopatrischen Populationen der Arten St. clavatus und St. rubicundus miteinander verpaart. Die daraus entstandenen Nachkommen wurden wiederum mit den Elternarten, aber auch untereinander verpaart. Für die Kreuzungsexperimente wurden jeweils ein Männchen und ein virginelles Weibchen der beiden Arten für mindestens eine Woche, aber oft auch länger, in einem Standardkäfig gehalten. Es wurde jeden Tag mehrmals kontrolliert, ob eine Kopulation stattgefunden hat. Das Weibchen hatte die Möglichkeit seine Eipakete direkt im Käfig abzulegen. Die Eipakete wurden gesammelt und für mindestens sechs Monate im Kühlschrank belassen. Die Nachkommen der verschiedenen Kreuzungen wurden in gleicher Art und Weise untersucht wie die anderen Individuen.

\subsection{Betrachtung von Klimadaten zur Untersuchung der ökologischen Nische}

Die klimatische Untersuchung der Habitate von St. rubicundus und St. clavatus soll Aufschluss über die ökologische Nische dieser Tiere, geben. Hierzu werden ausschließlich Wetterdaten benutzt, die aus dem Klimamodell Worldclim (Hijmans et al., 2005) stammen.

Worldclim ist ein Satz von interpolierten Datenobenflächen, sogenannten Layern, die auf Grundlage von Klimadaten von verschiedenen Wetterstationen, basieren. Die Datenoberflächen haben eine Auflösung von $1 \mathrm{~km}^{2}$. Es wurden für das Klimamodell meist Daten aus den Jahren 1950 bis 2005 von 24542 Wetterstationen weltweit (außer Antarktis), verwendet. In die Interpolation gehen zusammen mit den Wetterdaten der verschiedenen Stationen auch Längen- und Breitengrad, sowie die Höhe ein. Dadurch wird auf Klimaunterschiede 
in verschiedenen Höhenlagen Bezug genommen. Der Worldclim-Datensatz beinhaltet einen zusätzlichen Datensatz „BIOCLIM“. Diese klimatischen Daten werden hauptsächlich zur Untersuchung von ökologischen Nischen bei Tieren und Pflanzen verwendet (Waltari et al., 2007) und basieren auf Temperatur- und Niederschlagsdaten. Der Datensatz wurde in Form von Rasteroberflächen in ArcGIS dargestellt.

Für die vorliegende Arbeit wurde aus dem BIOCLIM Datensatz die Jahresdurchschnittstemperatur, die durchschnittliche Jahresniederschlagsmenge, die durchschnittliche Temperatur (in Grad Celsius) und die Niederschlagsmenge (in Millimeter) im Winter und Sommer, verwendet. Die Durchschnittswerte ergeben sich bei den Temperaturen aus den Mittelwerten aller wöchentlichen Durchschnittstemperaturen. Für die Bestimmung der mittleren Wochentemperatur wird der Durchschnitt aus minimaler und maximaler Temperatur in der jeweiligen Woche errechnet. Zur Bestimmung der mittleren Niederschlagsmengen wird der Durchschnitt aus den monatlichen Regenmengen der jeweiligen Jahre gebildet. Als „Winter“ und „Sommer“ werden die vier zusammenhängenden Monate bezeichnet, deren Durchschnittstemperatur am niedrigsten (Winter) oder am höchsten (Sommer) ist.

Trotz der flächendeckenden Oberflächenmodelle darf man nicht vergessen, dass viele der Klimadaten durch Interpolation entstanden sind und daher genähert sind. Nichtsdestotrotz ist dies die einzige Möglichkeit, flächendeckende Klimadaten auch von abgelegenen Regionen zu bekommen. 


\section{Ergebnisse}

In der vorliegenden Arbeit werden die Heuschrecken der Arten St. rubicundus, St. clavatus und die Individuen der Hybridzone zwischen beiden Arten morphologisch und ethologisch miteinander verglichen. Im ersten Teil wird der Flächeninhalt des Vorderflügels im Verhältnis zur Femurlänge vermessen. Sowie die Flügelform mit geometrischen Messpunkten charakterisiert. Im zweiten Teil werden die Antennen der Individuen vermessen und im letzten Teil die Gesänge der Männchen miteinander verglichen.

\subsection{Flügelmorphometrie}

Die Vermessung erfolgt mittels zweier unterschiedlicher morphometrischer Methoden. Zum einen wird der Flächeninhalt eines der beiden Vorderflügel im Bezug zur Femurlänge bestimmt. Zum anderen wird die Flügelform mit Hilfe von geometrischen Messpunkten („Landmarks“) ermittelt. Die erste der beiden Methoden gehört zu der klassischen Morphometrie, die zweite zur geometrischen Morphometrie. Somit kann im Detail auf Größen-, aber auch auf Formunterschiede eingegangen werden. Ziel dieser Methoden ist es, die Unterschiede und Gemeinsamkeiten der beiden Arten auf Populationsebene aufzuzeigen. Auf Grundlage dieser Daten können dann später die Individuen der TomarosPopulationen, und hier vor allem die intermediären Tiere, exakt charakterisiert werden.

Grundlage für die Einordnung der Tomaros-Tiere sind Vermessungen der Individuen aus den erwähnten Vergleichspopulationen, die zunächst auf inner- wie auf zwischenartliche Unterschiede hin zu untersuchen sind.

\subsubsection{Innerartliche Varianz}

\subsubsection{St. rubicundus der griechischen und slowenischen Populationen}

Im Hinblick auf den zwischenartlichen Vergleich mit St. clavatus wurde als erstes die innerartliche Varianz bestimmt. Innerhalb der St. rubicundus Populationen wurden zunächst die slowenische mit der griechischen Population verglichen, um festzustellen ob diese sich 
bereits unterscheiden. Hierzu wurden als erstes der Flächeninhalt sowie die Femurlänge von 107 Individuen $(67 \grave{\jmath} ; 40$ ) aus allopatrischen St. rubicundus-Populationen vermessen. Darauf folgte die morphometrische Analyse anhand von Messpunkten, die auf die Vorderflügel gesetzt wurden. Hierzu wurden die Vorderflügel von Individuen aus sechs St. rubicundus-Vergleichspopulationen morphometrisch vermessen. Dies waren Tiere aus Slowe-

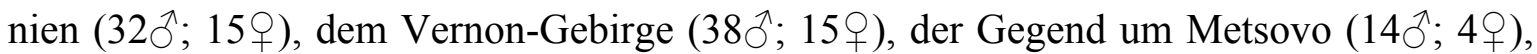
dem Mitsikeli-Gebirge (39ð; 25), dem Valtou-Gebirge $\left(12 \jmath^{\Uparrow} ; 3 \bigcirc\right)$ und der Nähe von Kalavrita auf der Peloponnes (14ð; 9 ㅇ).

Klassische Morphometrie: Die Flächeninhalte der Vorderflügel der slowenischen und griechischen St. rubicundus-Populationen unterscheiden sich nur sehr leicht (Tab. 1). Hierbei ist nur ein Teil der zuvor aufgeführten Die Vorderflügel der slowenischen St. rubicundus-Männchen sind mit durchschnittlich 69,67 $\mathrm{mm}^{2}$ nur geringfügig (ca. 1\%) größer als die der griechischen $\left(68,95 \mathrm{~mm}^{2}\right)$. Die durchschnittliche Femurlänge beträgt bei den Männchen der slowenischen Population 13,32 mm und ist damit um 1,36 mm länger als die der griechischen St. rubicundus-Männchen (11,96 mm). Dies weist darauf hin, dass die Männchen der slowenischen Population um etwa 11\% größer sind als die der griechischen. Demgegenüber ist die Zunahme der Vorderflügelgröße mit ca., 1\% minimal, d. h. die slowenischen St. rubicundus-Männchen haben in Bezug zur Körpergröße um ca. 10\% kleinere Vorderflügel. Beide Populationen unterscheiden sich hinsichtlich des Parameters Vorderflügelgröße signifikant $(\mathrm{p}<0,001)$ voneinander (Scheffé-Test).

Tab. 1 Durchschnittliche Größe des Vorderflügels und der Femurlänge und deren Verhältnis. Männchen und Weibchen der griechischen bzw. slowenischen St. rubicundus-Population

\begin{tabular}{|c|c|c|c|c|c|c|c|}
\hline Population & n & $\begin{array}{c}\text { Flächeninhalt } \\
\mathbf{m m}^{2}\end{array}$ & $\boldsymbol{\sigma}$ & $\begin{array}{c}\text { Femurlänge } \\
\text { mm }\end{array}$ & $\boldsymbol{\sigma}$ & $\begin{array}{c}\text { Flächeninhalt/ } \\
\text { Femurlänge }\end{array}$ & $\boldsymbol{\sigma}$ \\
\hline St. rubicundus (Slowenien) $\hat{\partial}$ & 16 & 69,67 & 3,71 & 13,32 & 0,36 & 5,23 & 0,25 \\
\hline St. rubicundus (Griechenland) ${ }^{\lambda}$ & 51 & 68,95 & 4,64 & 11,96 & 0,57 & 5,77 & 0,38 \\
\hline St. rubicundus (Slowenien) +9 & 10 & 50,96 & 4,40 & 16,06 & 0,83 & 3,18 & 0,29 \\
\hline St. rubicundus (Griechenland) & 30 & 49,62 & 3,49 & 14,14 & 0,70 & 3,51 & 0,27 \\
\hline
\end{tabular}

Betrachtet man die Weibchen der beiden St. rubicundus Populationen miteinander, ergibt sich ein ähnliches Bild. Auch hier besitzen die Vorderflügel der Weibchen der slowenischen Population mit einem durchschnittlichen Flächeninhalt von 50,96 $\mathrm{mm}^{2}$ einen nur geringfügig (2,7\%) größeren Vorderflügel als die der griechischen St. rubicundusPopulation $\left(49,62 \mathrm{~mm}^{2}\right)$. Größer fallen die Unterschiede bei den Femurlängen aus. Sie betragen bei den slowenischen St. rubicundus-Weibchen 16,06 $\mathrm{mm}$ und bei den griechi- 
schen $14,14 \mathrm{~mm}$, d. h. erstere sind um ca. 13,6\% länger. Setzt man, wie oben bei den Männchen, die Flügelgröße in Bezug zur Femurlänge, die ein Maß für die Körpergröße ist, so ergibt sich auch hier, dass die Vorderflügel der slowenischen Tiere relativ kleiner als die der griechischen sind. Der Unterschied ist signifikant ( $p<0,001$ Scheffé).

\section{Geometrische Morphometrie:}

Nachdem die Vorderflügel mit klassischen Methoden vermessen worden sind, wird nunmehr mit morphometrischen Methoden die Flügelform ermittelt. Dies geschieht mit Hilfe von morphologisch-geographischen Koordinaten (Landmarks); siehe hierzu Kap. 2.4.1 in Material und Methoden.

Wie dort bereits beschrieben, werden die mit morphologisch-geographischen Koordinaten (Landmarks) markierten Vorderflügel der Individuen in Gruppen zusammengefasst und durch Hauptkomponentenanalyse und kanonischer Varianzanalyse miteinander verglichen.

Bei den folgenden Vergleichen wurde stets zunächst eine Hauptkomponentenanalyse mit dem Programm PVAgen6 durchgeführt, um mögliche morphologische Unterschiede aufzuzeigen. Danach wurde mit Hilfe des Programms CVAgen6 eine kanonische Varianzanalyse durchgeführt.

Die Hauptkomponentenanalyse zeigt bei dem innerartlichen Vergleich eine Gesamtvariation der ersten beiden Variationsebenen von 44 \% (PV1: 28 \%; PV2: $16 \%$ ) bei den Männchen und 53,0\% (PV1: 31,7\%; PV2 22,3) bei den Weibchen. Nach Auftragung dieser beiden Ebenen wird deutlich, dass sich die slowenischen St. rubicundus - gleiches gilt, auch für jene aus dem Engadin - von den griechischen unterscheiden (Abb. 26).

Mit Hilfe der CVA/MANOVA-Methode wurde nun auf Signifikanz geprüft. Hierzu wurde eine MANOVA-Analyse auf Grundlage der Werte aus der kanonischen Varianzanalyse durchgeführt (Abb. 27). Die Analyse errechnet den Wilks- $\lambda$-Wert, der die Größe des Unterschieds zwischen den gewählten Gruppen angibt. Umso näher Wilks $\lambda$ an 1 ist, umso kleiner ist der Unterschied zwischen den Gruppen. Der CVA/MANOVA-Test zeigt in der Tat eine signifikante Differenz von $\mathrm{p}<0,001$ zwischen den griechischen St. rubicundusMännchen und den slowenischen. Mit einem Wilks- $\lambda$-Wert von 0,2752 sind die Gruppen gut voneinander getrennt. Ähnliche Werte zeigen ebenfalls die Weibchen beider Gruppen 
(Abb. 28 und Abb. 29). Sie unterscheiden sich ebenfalls signifikant $(p<0,001)$ und haben einen Wilks- $\lambda$-Wert von 0,2086.

Um mögliche Fehler, die durch die Einteilung in Gruppen entstehen können, zu erkennen, bot sich der Jackknife-Test an. Hierbei wird eine definierte Menge (zwischen 10 und 50 Prozent) aus dem Datensatz genommen und als „Unbekannte“ definiert. Danach wird überprüft, inwieweit sich die Gruppeneinteilungen ändern (näheres in Kap 2.4.5). Der Jackknife-Test zeigte eine Wiedereinordnung von 91,3 \% der Individuen bei einer Ausschlussrate von $10 \%$ und von 88,5\% bei einer Ausschlussrate von $50 \%$. Bei den Weibchen wurden $90,2 \%$ bei $90 \%$ und $59,9 \%$ bei $50 \%$ Ausschluss wieder eingeordnet. Somit ist diese Einteilung auch bei den Weibchen gerechtfertigt.

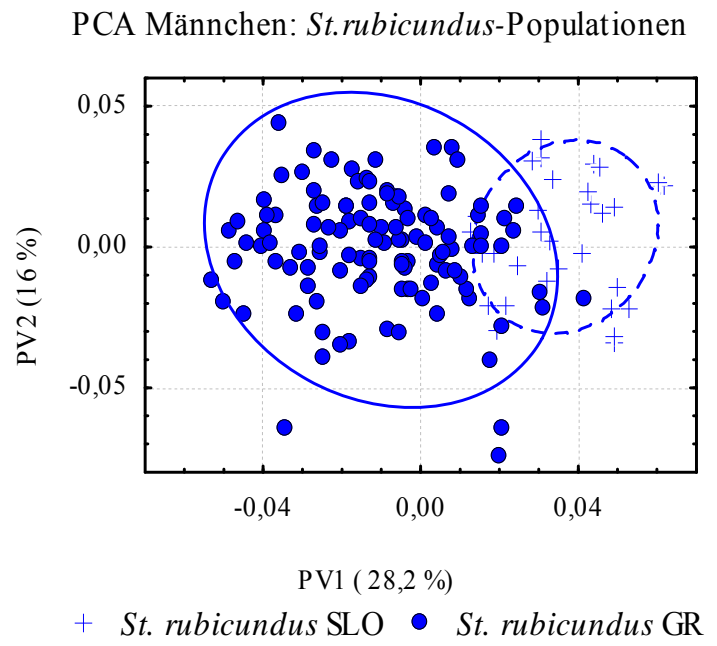

Abb. 26 Hauptkomponentenanalyse der Vorderflügel der slowenischen und griechischen St.rubicundus-Populationen. (Männchen)
CVA -Männchen: St. rubicundus-Populationen

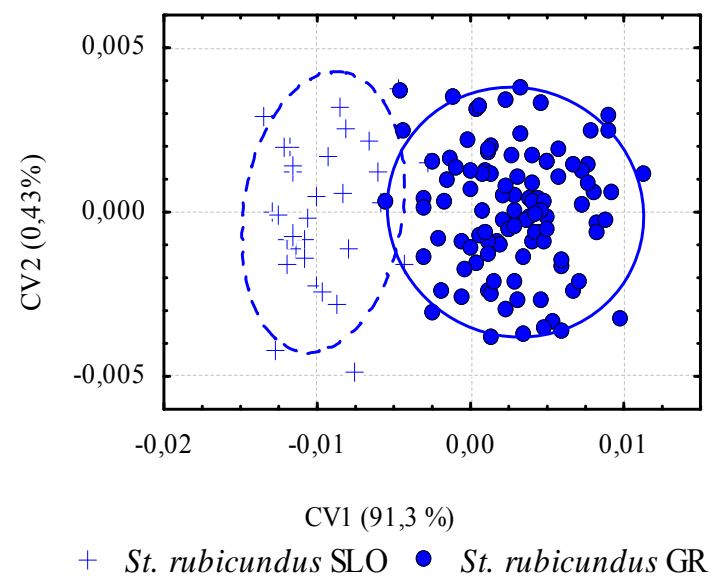

Abb. 27 Kanonische Varianzanalyse der Vorderflügel der slowenischen und griechischen St.rubicundus-Populationen. (Männchen)

Dies bedeutet, dass die Einteilung der Individuen in Populationen bei der kanonischen Varianzanalyse auf Grundlage ihrer Fundorte und Artmerkmale mit sehr hoher Wahrscheinlichkeit richtig ist, da die Individuen der beiden Gruppen selbst bei Ausschluss von $50 \%$ der Daten durch den Jackknife-Test die Populationszuordnung hoch signifikant bleibt. 
PVA Weibchen: St. rubicundus-Population

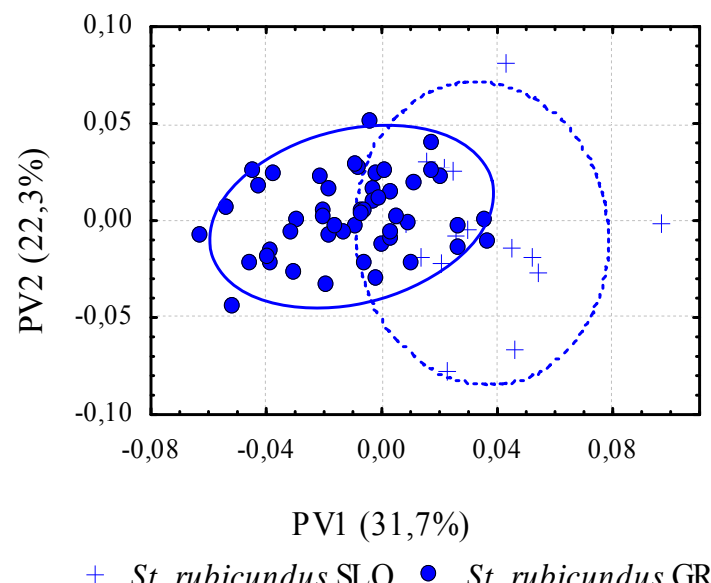

Abb. 28 Hauptkomponentenanalyse der Vorderflügel der slowenischen und griechischen St. rubcundus-Populationen (Weibchen) .
CVA-Weibchen: St. rubicundus-Populationen

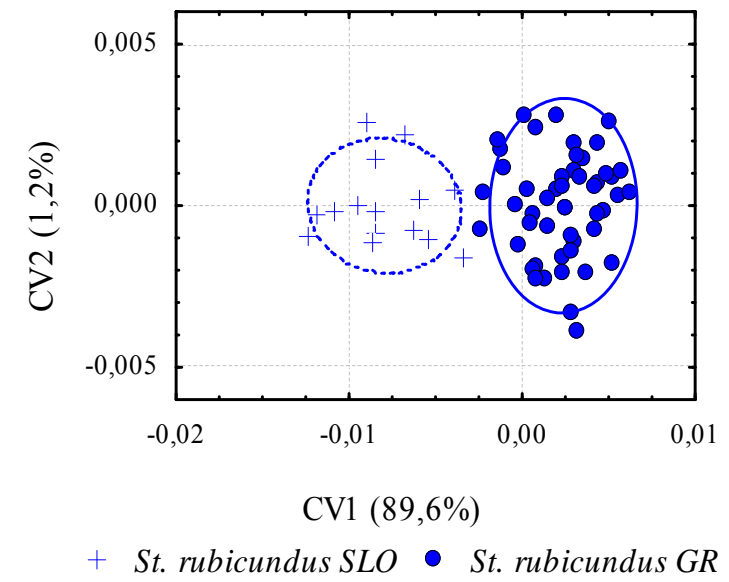

Abb. 29 Kanonische Varianzanalyse der Vorderflügel der slowenischen und griechischen St.rubicundus-Populationen (Weibchen).

\subsubsection{Vergleich zwischen den griechischen St. rubicundus Populationen}

Nachdem zunächst die griechischen St. rubicundus-Populationen als Ganzes gegenüber der slowenischen Population auf Unterschiede hinsichtlich der Flügelgröße und -gestalt geprüft worden waren, werden nunmehr die griechischen Populationen einzeln untersucht und miteinander verglichen.

\section{Klassische Morphometrie:}

Für die Bestimmung des Flächeninhalts des Vorderflügels der Männchen und Weibchen der einzelnen St. rubicundus-Populationen Griechenlands wurden Individuen aus der Popu-

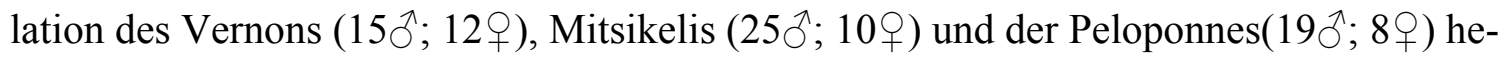
rangezogen (Tab. 2).

Tab. 2 Durchschnittliche Größe des Vorderflügels und der Femurlänge und deren Verhältnis. Männchen und Weibchen verschiedener St. rubicundus-Populationen Griechenlands.

\begin{tabular}{|c|c|c|c|c|c|c|c|}
\hline Population & $\mathbf{n}$ & $\begin{array}{c}\text { Flächeninhalt } \\
\text { mm }^{2}\end{array}$ & $\boldsymbol{\sigma}$ & $\begin{array}{c}\text { Femurlänge } \\
\text { mm }\end{array}$ & $\boldsymbol{\sigma}$ & $\begin{array}{c}\text { Flächeninhalt/ } \\
\text { Femurlänge }\end{array}$ & $\boldsymbol{\sigma}$ \\
\hline St. rubicundus (Vernon) ${ }^{\lambda}$ & 15 & 67,12 & 4,24 & 11,89 & 0,45 & 5,65 & 0,34 \\
\hline St. rubicundus (Mitsikeli) ${ }^{\lambda}$ & 25 & 69,85 & 4,65 & 12,20 & 0,56 & 5,73 & 0,38 \\
\hline St. rubicundus (Peloponnes) & 19 & 69,42 & 4,81 & 11,51 & 0,43 & 6,03 & 0,34 \\
\hline St. rubicundus (Vernon) $q$ & 12 & 51,30 & 4,31 & 14,44 & 0,53 & 3,56 & 0,30 \\
\hline St. rubicundus (Mitsikeli) $q$ & 10 & 47,48 & 2,03 & 14,24 & 0,85 & 3,34 & 0,22 \\
\hline St. rubicundus (Peloponnes) $q$ & 8 & 49,76 & 2,15 & 13,57 & 0,34 & 3,67 & 0,18 \\
\hline
\end{tabular}


Die Vorderflügelgröße bei den Männchen der Mitsikeli-Population $\left(69,85 \mathrm{~mm}^{2}\right)$ und der Peloponnes-Population $\left(69,42 \mathrm{~mm}^{2}\right)$ unterscheidet sich kaum voneinander. Geringfügig kleiner sind mit $67,12 \mathrm{~mm}^{2}$ die Vorderflügel St. rubicundus-Männchen der VernonPopulation. Betrachtet man die durchschnittliche Femurlänge der Männchen, so variieren die Durchschnittswerte bei den einzelnen Populationen zwischen 11,51mm (Peloponnes), 11,89 mm (Vernon) und 12,20mm (Mitsikeli). Daraus ergeben sich für das Verhältnis von Flächeninhalt/Femurlänge durchschnittliche Werte der Männchen der einzelnen Populationen zwischen 5,63 (Vernon), 5,73 (Mitsikeli) und 6,03 (Peloponnes). Unterzieht man die Gruppen einem Signifikanztest, so unterscheiden sich die Mitsikeli-Population und die Vernon-Population nicht voneinander und die Vernon-Population von der PeleponnesPopulation nur schwach signifikant $(\mathrm{p}=0,039)$.

Mit Größen von 51,30 $\mathrm{mm}^{2}$ (Vernon), 47,48 $\mathrm{mm}^{2}$ (Mitsikeli) und 49,76 $\mathrm{mm}^{2}$ (Peloponnes) sind die Flügel der Weibchen kleiner als die der Männchen, während die Femora mit 14,44 mm (Vernon), 14,24 mm (Mitsikeli) und 13,57 mm (Peloponnes) um ca. $2 \mathrm{~mm}$ bis $3 \mathrm{~mm}$ länger sind. Bildet man das Verhältnis von Vorderflügel-Flächeninhalt und Femurlänge, so ergeben sich Werte von 3,56 (Vernon), 3,34 (Mitsikeli) bis 3,67 (Peloponnes). Hierbei unterscheiden sich die Weibchen der Peloponnes- und die der Vernon-Population nicht signifikant voneinander. Zwischen den Weibchen der Mitsikeli-Population und denen der St. rubicundus-Population aus dem Vernon-Gebirge gibt es einen leicht signifikanten Unterschied $(\mathrm{p}=0,033$, Scheffé $)$

Geometrische Morphometrie: Zusätzlich zur Bestimmung der Flügelgröße und der Femurlänge werden nun die Vorderflügel geometrisch vermessen und miteinander verglichen.

Die Hauptkomponentenanalyse ergibt, dass sich die St. rubicundus-Männchen der verschiedenen griechischen Standorte hinsichtlich der Flügelgestalt offensichtlich nicht voneinander unterscheiden. Nun zeigt aber der CVA/MANOVA-Test der kanonischen Varianzanalyse drei signifikante Variationsebenen zwischen den fünf Gruppen (Abb. 30). Das würde bedeuten, dass laut dieser Rechnung vier der fünf Gruppen sich signifikant voneinander unterscheiden. Die Überprüfung mittels des Jackknife-Tests hält aber dieser Einschätzung nicht Stand: Lediglich 53,8 \% der Werte bleiben signifikant und behalten ihre Einteilung, wenn 10\% der Werte ausgeschlossen werden. Der Fehler vergrößert sich bei einer Ausschlussrate von 50\% der Daten, denn danach sinkt der Anteil richtiger und signi- 
fikanter Werte auf 40,2 Prozent. Auch die Weibchen dieser Populationen unterscheiden sich nicht signifikant voneinander.

Als Fazit ist demnach festzuhalten, dass sich die griechischen St. rubicundus -Populationen nicht signifikant voneinander unterscheiden und somit jede von ihnen als Referenz für den zwischenartlichen (Abb. 30) Vergleich mit St. clavatus und die Einordnung der TomarosTiere herangezogen werden können. Die slowenischen und die alpinen Populationen werden aufgrund des signifikanten Unterschieds gegenüber den griechischen Populationen hingegen hierfür nicht verwendet.

CVA Männchen: St. rubicundus -Population Griechenland

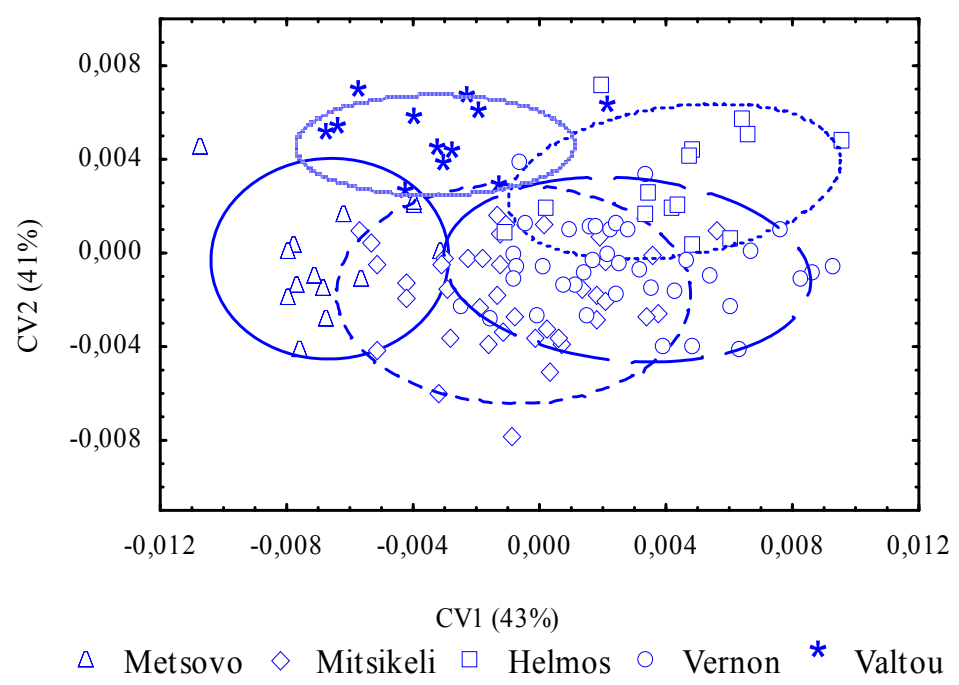

Abb. 30 Kanonische Varianzanalyse der Vorderflügel der griechischen St .rubicundus-Populationen Männchen

\subsubsection{St. clavatus}

Als Referenz für die Tomaros-Tiere von St. clavatus steht einzig die Xerovouni-Population zur Verfügung, womit sich, anders als bei St. rubicundus, innerartliche Vergleiche verschiedener Populationen erübrigen. Es wurden für die klassische 44 und für die geometrische Morphometrie 147 zufällig ausgewählte Individuen herangezogen, die von neun Fundorten (GPS-Punkten) auf dem Xerovouni stammten.

Klassische Morphometrie: Die Männchen dieser Population haben einen durchschnittlichen Vorderflügel-Flächeninhalt von $30,61 \mathrm{~mm}^{2}$ und eine durchschnittliche Femurlänge von 11,03mm (Tab. 3). Bei Weibchen dieser Art wurde ein durchschnittlicher Vorderflügelflächeninhalt von $34,45 \mathrm{~mm}^{2}$ und eine mittlere Femurlänge von $14,59 \mathrm{~mm}$ gemessen. Es 
ergibt sich daraus ein Flächeninhalt/Femurlänge-Verhältnis für die St. clavatus-Männchen von 2,78 und für die Weibchen von 2,36.

Die Weibchen haben somit einen um ca. $4 \mathrm{~mm}^{2}$ größeren Vorderflügel und einen um ca. 3,5 mm längeren Femur als die Männchen derselben Art. Dies spiegelt sich nur bedingt im Verhältnis von Flächeninhalt und Femurlänge wider. Dies ist bei den Männchen größer als bei den Weibchen. Die Männchen haben im Vergleich zur Femurlänge einen etwas größeren Vorderflügel als die Weibchen.

Tab. 3 Durchschnittliche Größe des Vorderflügels und Femurlänge der Männchen und Weibchen der Arten St. clavatus

\begin{tabular}{|c|c|c|c|c|c|c|c|}
\hline Population & $\mathbf{n}$ & $\begin{array}{c}\text { Flächeninhalt } \\
\mathbf{m m}^{\mathbf{2}}\end{array}$ & $\boldsymbol{\sigma}$ & $\begin{array}{c}\text { Femurlänge } \\
\mathbf{m m}\end{array}$ & $\boldsymbol{\sigma}$ & $\begin{array}{c}\text { Flächeninhalt/ } \\
\text { Femurlänge }\end{array}$ & $\boldsymbol{\sigma}$ \\
\hline St. clavatus (Xerovouni) $\delta$ & 27 & 30,61 & 2,28 & 11,03 & 0,56 & 2,78 & 0,18 \\
\hline St. clavatus (Xerovouni) $\not$ & 17 & 34,45 & 2,59 & 14,59 & 0,82 & 2,36 & 0,11 \\
\hline
\end{tabular}

\subsubsection{Vergleich zwischen St. rubicundus und St. clavatus}

Klassische Morphometrie: Für die Untersuchung des Vorderflügelflächeninhalts und der Femurlänge der beiden Arten wurden Individuen der griechischen St. rubicundusPopulationen und der St. clavatus Population des Xerovouni verwendet (Tab. 4). Die Vorderflügel der Männchen der Art St. rubicundus haben einen durchschnittlichen Flächeninhalt von $68,95 \mathrm{~mm}^{2}$ und somit einen um $38,34 \mathrm{~mm}^{2}$ größeren Vorderflügelflächeninhalt als die St. clavatus $\left(30,61 \mathrm{~mm}^{2}\right)$. Der Femur der Individuen der Art St. rubicundus ist mit durchschnittlich 11,96 mm Länge um 0,93 mm länger als die mittlere Femurlänge von St. clavatus (11,03 mm). Dementsprechend ist ebenfalls das Verhältnis Femur/Flächeninhalt der Männchen von St .rubicundus mit durchschnittlich 5,77 um einiges größer als das der Individuen der Art St. clavatus. Somit besitzen die Männchen der Art St. rubicundus einen mehr als doppelt so großen Vorderflügel, einen etwas längeren Femur, daraus resultiert ein ebenfalls doppelt so großes Verhältnis beider Parameter.

Tab. 4 Durchschnittliche Größe des Vorderflügels und Femurlänge der Männchen und Weibchen der Arten St. rubicundus und St. clavatus

\begin{tabular}{|c|c|c|c|c|c|c|c|}
\hline Population & $\mathbf{n}$ & $\begin{array}{c}\text { Flächeninhalt } \\
\text { mm }^{2}\end{array}$ & $\boldsymbol{\sigma}$ & $\begin{array}{c}\text { Femurlänge } \\
\text { mm }\end{array}$ & $\boldsymbol{\sigma}$ & $\begin{array}{c}\text { Flächeninhalt/ } \\
\text { Femurlänge }\end{array}$ & $\boldsymbol{\sigma}$ \\
\hline St. rubicundus (Griechenland) ${ }^{\lambda}$ & 51 & 68,95 & 4,64 & 11,96 & 0,57 & 5,77 & 0,38 \\
\hline St. clavatus (Xerovouni) $\partial^{\lambda}$ & 27 & 30,61 & 2,28 & 11,03 & 0,56 & 2,78 & 0,18 \\
\hline St. rubicundus (Griechenland) $q$ & 30 & 49,62 & 3,49 & 14,14 & 0,70 & 3,51 & 0,27 \\
\hline St. clavatus (Xerovouni) $q$ & 17 & 34,45 & 2,59 & 14,59 & 0,82 & 2,36 & 0,11 \\
\hline
\end{tabular}


Griechische St. rubicundus Weibchen haben einen durchschnittlichen Vorderflügelflächeninhalt von 49,62 $\mathrm{mm}^{2}$. Demnach ist der Flächeninhalt dieser Weibchen um 15,17 $\mathrm{mm}^{2}$ größer, als der der St. clavatus Weibchen. Die Femurlänge der St. rubicundus Weibchen beträgt durchschnittlich $14,14 \mathrm{~mm}$ und ist daher um 0,45 $\mathrm{mm}$ kürzer als bei den Weibchen der Art St. clavatus (14,59 mm). Hieraus ergibt sich ein Verhältnis von Flächeninhalt und Femurlänge von 3,51 bei den Weibchen der Art St. rubicundus. Somit ist dieses um einiges größer als bei den Weibchen der Art St. clavatus. Auch die Weibchen beider Arten unterscheiden sich signifikant ( $p>0,001)$ voneinander. Aber im Gegensatz gibt es keinen großen Unterschied in der Femurlänge der Weibchen. Somit sind die Weibchen beider Arten aller Wahrscheinlichkeit nach ähnlich groß.

Geometrische Morphometrie: Wie bei der Untersuchung der St. rubicundus-Populationen werden Stichproben aus den zu untersuchenden Populationen entnommen und mit Hilfe der Hauptkomponenten- und kanonischen Varianzanalyse, miteinander verglichen. In Abb. 32 ist zu sehen, dass sich bei der Hauptkomponentenanalyse die beiden Arten zwei voneinander getrennten Gruppen zuordnen lassen. Die Achsen 1 (PV1) und 2 (PV2) dieser Analyse repräsentieren ca. $95 \%$ der Gesamtvariation, hierbei repräsentiert die Achse 1 (PV1) mit 93,43 \% den Großteil der Gesamtvarianz. Mit Hilfe des Programms TPSrelw wurden die relativen Varianzen der homologen anatomischen Punkte (Landmarken) der Vorderflügel ermittelt (Abb. 31). Hierzu diente der Vergleich zwischen den Punkten der Vorderflügel der Männchen beider Arten. Die Punkte 4; 10; 11; 12 und 13 zeigen bei diesem zwischenartlichen Vergleich gegenüber den anderen Punkten eine weitaus größere Varianz (Abb. 31). Die kleinste Varianz ist bei den Punkten 3; 8 und 9 zu beobachten.

Auch die Weibchen zeigen bei der Hauptkomponentenanalyse ein ähnliches Muster wie die Männchen. Einzig die Varianz der ersten Hauptkomponentenachse (93,43\% Männchen, 91,96\% Weibchen) unterscheidet sich etwas von denen der Männchen. 


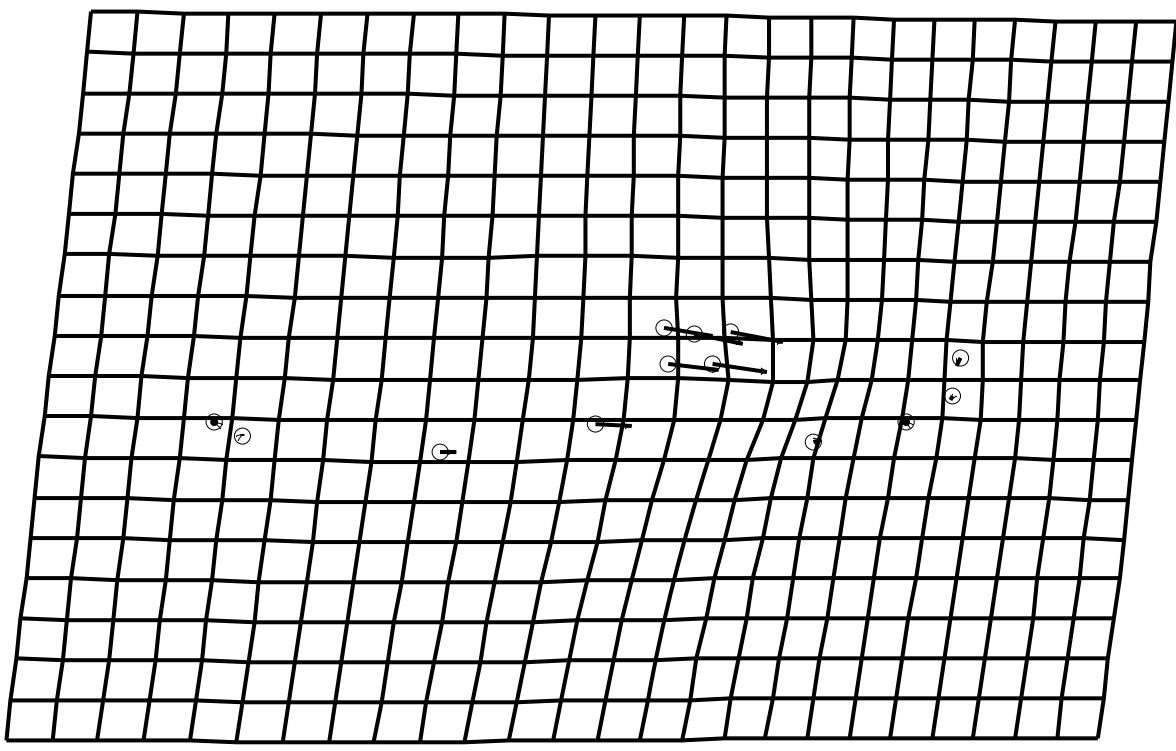

\begin{tabular}{|c|c|}
\hline LM & Varianz \\
\hline $\mathbf{1}$ & 0,024 \\
\hline $\mathbf{2}$ & 0,032 \\
\hline $\mathbf{3}$ & 0,004 \\
\hline $\mathbf{4}$ & 0,138 \\
\hline $\mathbf{5}$ & 0,015 \\
\hline $\mathbf{6}$ & 0,046 \\
\hline $\mathbf{7}$ & 0,024 \\
\hline $\mathbf{8}$ & 0,006 \\
\hline $\mathbf{9}$ & 0,001 \\
\hline $\mathbf{1 0}$ & 0,112 \\
\hline $\mathbf{1 1}$ & 0,372 \\
\hline $\mathbf{1 2}$ & 0,078 \\
\hline $\mathbf{1 3}$ & 0,144 \\
\hline
\end{tabular}

Abb. 31 Vektoren und Landmarken-Varianzen zwischen St. clavatus und St. rubicundus Männchen. Erstellt mit TPSelw. LM: Landmarken.

Mit Hilfe der kanonischen Varianzanalyse (Kapitel 2.4.4) kann nun der Unterschied zwischen den Gruppen bzw. Arten untersucht werden (Abb. 33). Der MANOVA Test zwischen den St. clavatus und St. rubicundus Männchen ergab eine Signifikanz von $\mathrm{p}<0,001$, sowie ein Wilks- $\lambda$-Wert von 0,0070. Die Jackknife-Werte liegen bei 99,8 \% (10\% weggelassene Werte) und bei 97,7 \% (50\% weggelassene Werte) bei 1000 Wiederholungen. Also ist die Zuordnung der Tiere zu diesen beiden Gruppen sehr konsistent.

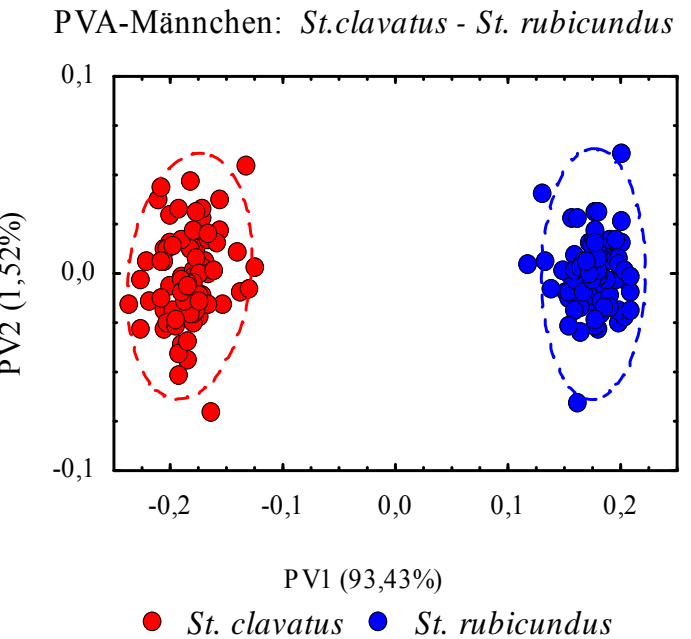

Abb. 32 Hauptkomponentenanalyse der Vorderflügel der St. clavatus- und St. rubicundus- Männchen

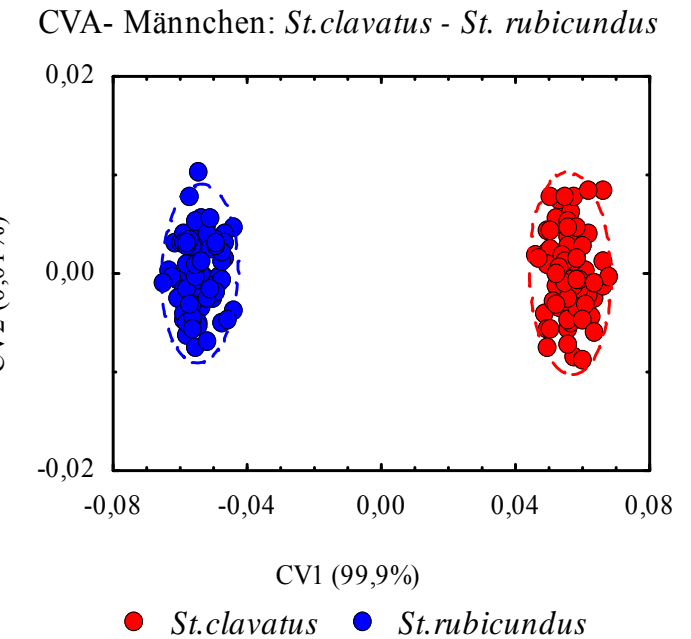

Abb. 33 Kanonische Varianzanalyse der Vorderflügel der St. clavatus- und St. rubicundus- Männchen

Die Untersuchung der Vorderflügel der Weibchen beider Arten mittels Hauptkomponentenanalyse ergab eine Gesamtvarianz der ersten beiden Variationsachsen (PV1 und PV2) 
von zusammen 93,82\%. Auch hier trennen sich die beiden Populationen gut voneinander ab (Abb. 34) und auch bei der kanonischen Varianzanalyse bestätigt sich dieses Bild (Abb. 35). Zwischen den Weibchen beider Populationen wurde beim CVA/MANOVA-Test ein Wilks- $\lambda$-Wert von 0,0086 ermittelt sowie eine Signifikanz von $p<0,001$. Auch hier unterstützt der Jackknife-Test, mit einer Beständigkeit von 97,6 \% bei 10\% weggelassener Werte und von 89,6 Prozent bei 50\% weggelassener Werte diese Einteilung. Somit ist der jeweilige zwischenartliche Unterschied zwischen den Männchen und zwischen den Weibchen der beiden Arten gut gesichert und in etwa gleich.

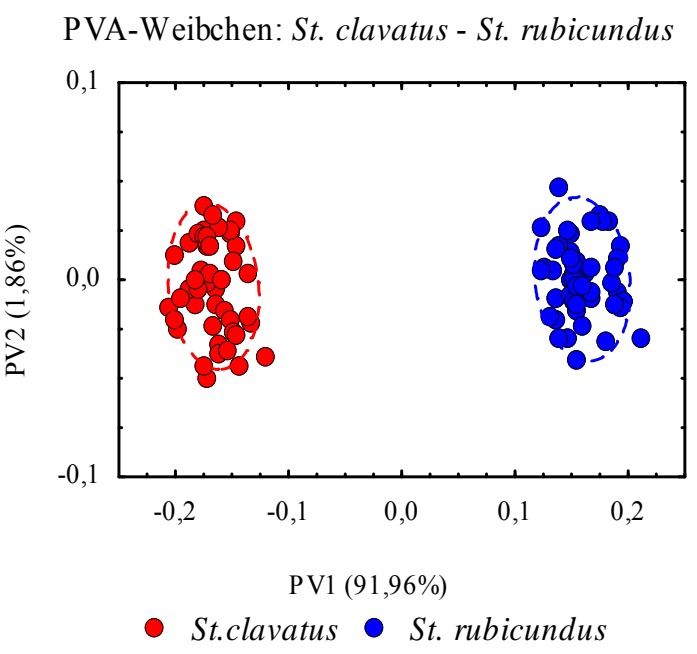

Abb. 34 Hauptkomponentenanalyse der Vorderflügel der St. clavatus- und St. rubicundus- Weibchen

\section{CVA- Weibchen: St. clavatus - St. rubicundus}

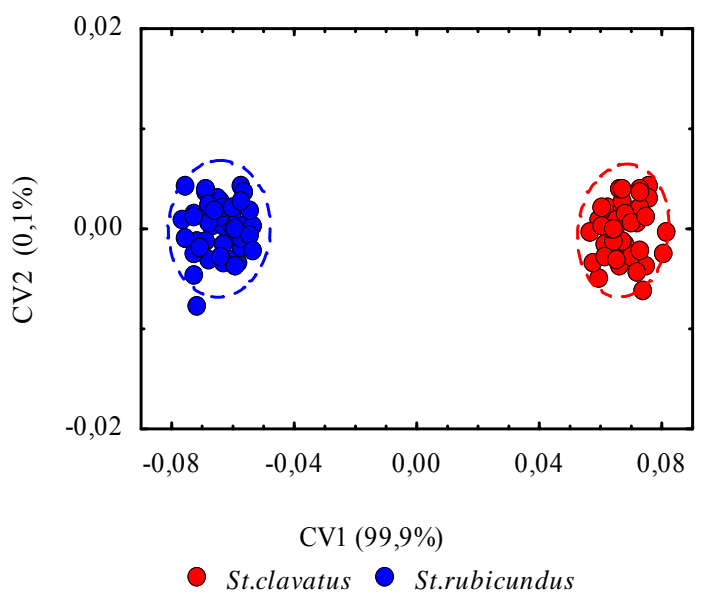

Abb. 35 Kanonische Varianzanalyse der Vorderflügel der St. clavatus- und St. rubicundus- Weibchen

Nachdem der interspezifische Vergleich gezeigt hat, dass sowohl die Männchen als auch die Weibchen beider Arten sich jeweils hoch signifikant unterscheiden.

\subsubsection{Einordnung der zwischenartlichen Varianz mit Hilfe von Außengruppen.}

Die bisherigen Analysen haben zwar ergeben, dass mit Hilfe der geometrisch morphometrischen Methode die beiden Arten St. rubicundus und St. clavatus signifikant voneinander zu trennen sind, jedoch ist es notwendig, die so gewonnenen Daten mit denen von Individuen anderer Stenobothrus-Arten zu vergleichen.

Hierzu wurden insgesamt vier Stenobothrus-Arten als Außengruppen herangezogen und deren Vorderflügel vermessen: St. fischeri $(\mathrm{n}=12)$, St. lineatus $(\mathrm{n}=9)$, St. nigromaculatus $(\mathrm{n}=6)$ St. stigmaticus $(\mathrm{n}=7)$ 
Bei der Hauptkomponentenanalyse zeigte sich, dass St. clavatus, St. stigmaticus und St. nigromaculatus sowie St. fischeri und St. lineatus jeweils eine Gruppe bilden. St. rubicundus grenzte sich hingegen von den anderen Gruppen ab. Von den 22 Achsen der Hauptkomponentenanalyse beinhalten die ersten beiden Achsen (PV1 und PV2) mit $93 \%$ den größten Teil der Gesamtvarianz.

Die daraus zu ziehende Schlussfolgerung wäre, dass die Individuen, die sich jeweils zu einer Gruppe zusammenfügen, morphologisch ähnlich sind. Dies ist jedoch nicht berechtigt, denn der bei der kanonischen Varianzanalyse (Abb. 37) durchgeführte CVA/MANOVA-Test zeigt, dass bei diesem zwischenartlichen Vergleich fünf signifikante Variationsebenen (Abb. 38) zu finden sind. Mit diesem Test können also alle sechs Arten auf Basis der Flügelmorphometrie signifikant unterschieden werden. Auch der JackknifeTest, der die Beständigkeit der Einteilung auf Artebene überprüfen soll, bestätigt dies mit 97,4\% bei 10\% Ausschluss der Daten bzw. 94\% bei 50\% Ausschluss.

Die Darstellung der Achsen CV2 und CV3 zeigt deutlich, dass St. rubicundus und St. clavatus sich von den vier Außengruppen abgrenzen (Abb. 38). St. rubicundus und St. clavatus besitzen einige wenige gemeinsame Merkmale, mit denen sich diese beiden Arten von den anderen Arten abgrenzen. Somit kann die Untersuchung der Zwischenformen auf dem Tomaros ausschließlich auf Grundlage der Elternarten, also in diesem Fall St. rubicundus und St. clavatus, erfolgen. 
PCA-Männchen: Stenobothrus- Arten

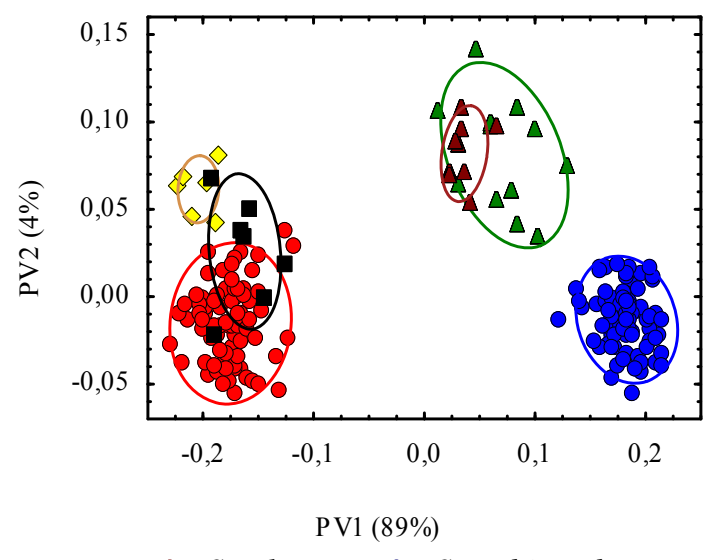

- St. clavatus - St. rubicundus

\ St. fischeri $\mathbf{\Delta}$ St. lineatus

St. nigromaculatus - St. stigmaticus

Abb. 36 Hauptkomponentenanalyse von St. clavatus, St. rubicundus und einiger weiterer Stenobothrus-Arten

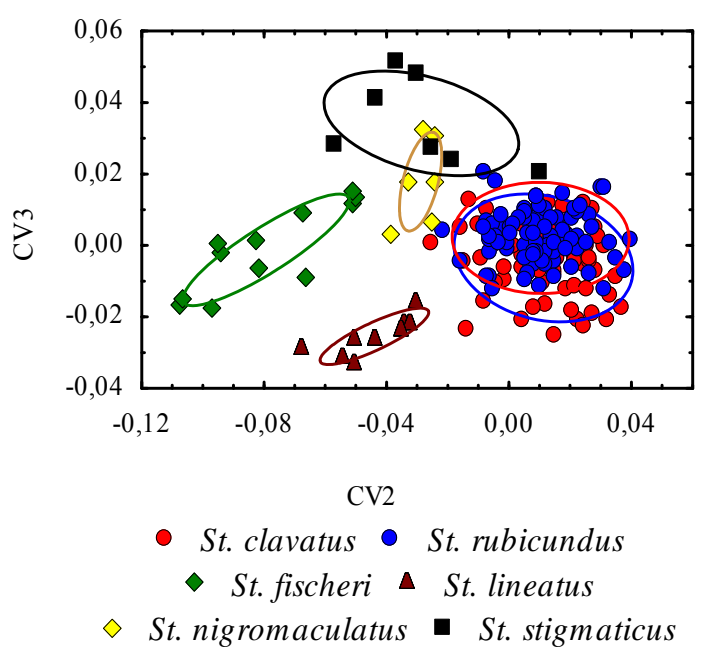

CVA-Männchen: Stenobothrus- Arten

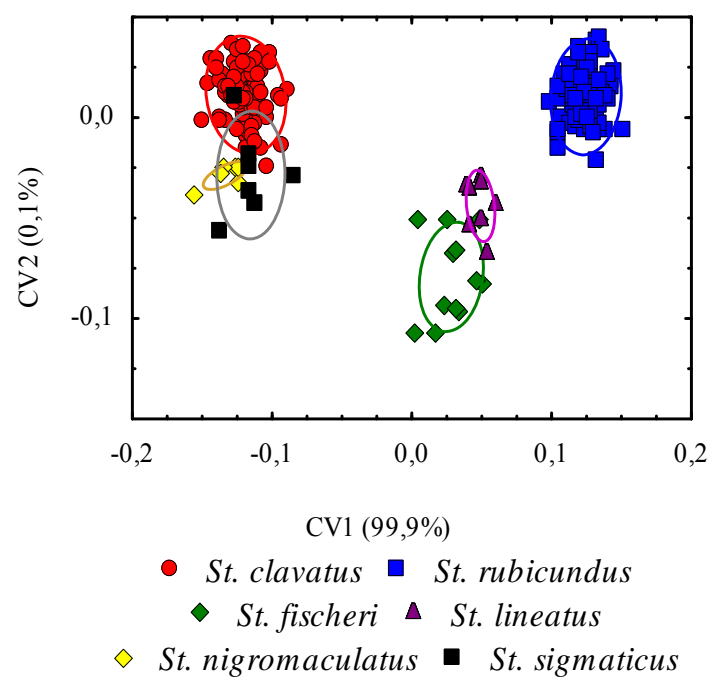

Abb. 37 Kanonische Varianzanalyse von St. clavatus, St. rubicundus und einiger weiterer Stenobothrus-Arten. Kanonische Varianzebene CV1 und $\mathrm{CV} 2$

\begin{tabular}{|c|c|c|c|c|}
\hline $\begin{array}{c}\text { CVA } \\
\text { Achse }\end{array}$ & Wilks $\boldsymbol{\lambda}$ & $\begin{array}{c}\text { Chi- } \\
\text { Quadrat }\end{array}$ & Df & $\mathbf{p}$ \\
\hline $\mathbf{1}$ & 0,0005 & 1424,36 & 30 & $\mathrm{p}>0,001$ \\
\hline $\mathbf{2}$ & 0,0437 & 567,50 & 20 & $\mathrm{p}>0,001$ \\
\hline $\mathbf{3}$ & 0,2064 & 293,45 & 12 & $\mathrm{p}>0,001$ \\
\hline $\mathbf{4}$ & 0,4539 & 146,91 & 6 & $\mathrm{p}>0,001$ \\
\hline $\mathbf{5}$ & 0,8852 & 22,67 & 2 & $\mathrm{p}>0,001$ \\
\hline
\end{tabular}

Abb. 38 Hauptkomponentenanalyse von St. clavatus, St. rubicundus und einiger weiterer Stenobothrus-Arten Kanonische Varianzebene CV1 und CV2 (links). Tabelle rechts: Werte der einzelnen Variationsebenen.

Fazit: Die bisherigen Analysen aufgrund der Morphometrie der Vorderflügel von Individuen verschiedener Stenobothrus-Populationen haben ergeben, dass St. rubicundus und St. clavatus zum einen eindeutig von anderen Stenobothrus-Arten (St. fischeri, St. lineatus, St. nigromaculatus, St. stigmaticus ) zu trennen sind, zum anderen aber auch signifikant voneinander unterschieden sind. Was St. rubicundus betrifft, so unterscheiden sich wiederum die griechischen von den alpinen und slowenischen Populationen. Als Referenzgruppen zur nunmehr vorzunehmenden Einordnung der Tomaros-Tiere werden die griechischen St. 
rubicundus-Populationen sowie, was St. clavatus betrifft, die Xerovouni-Population dieser Art herangezogen.

\subsubsection{Populationen des Tomarosgebirges -klassische Morphometrie-}

Die Individuen des Tomaros werden in diesem Kapitel zunächst mit Hilfe der klassischen Morphometrie untersucht. Der geometrisch morphologische Vergleich der Tiere des Tomaros folgt detailliert in den späteren Kapiteln 3.1.6.3 und 3.1.6.5.

Klassische Morphometrie: Für die Untersuchung des Flächeninhalts der Vorderflügel und der Femurlänge der Populationen des Tomaros werden die Individuen aufgrund ihrer Fundorte und offensichtlicher morphologischer Merkmale in vier Gruppen unterteilt (Tab. 5). Dies sind auf dem Südmassiv des Tomaros (1) die St. clavatus-ähnlichen Individuen des Südhanges (42§; 24+) und (2) die St. rubicundus-ähnlichen Individuen des Osthanges (23ð’; 17 ) ), ferner (3) intermediäre Individuen, die im Bereich der Überschneidungszone auf dem Sattel des Südtomaros $(80 \hat{\jmath} ; 10$ \% $)$ zu finden sind und (4) St. clavatus-ähnliche Individuen des Nordtomaros $(17 \hat{\jmath} ; 16$ ㅇ). Alle Populationen werden mit den allopatrischen St. rubicundus- bzw.- St. clavatus-Populationen außerhalb des Tomaros verglichen.

Tab. 5 Fundorte (GPS) der Populationen auf dem Tomaros

\begin{tabular}{|c|c|c|c|c|}
\hline Fundorte & GPS & Art & $\hat{0}$ & 온 \\
\hline Südhang des Südtomaros & START;1ST;OP;LIAL3 & St. clavatus & 41 & 13 \\
\hline Osthang des Südtomaros & LCU; OH4-5;N2;OH3 & St. rubicundus & 36 & 17 \\
\hline Sattel (Südtomaros) & LIGIP;HYBGUT & Intermediäre Indiv. & 67 & 10 \\
\hline Norden des Nordtomaros & NT1;NT2;NTA;NTB & St.clavatus & 17 & 16 \\
\hline
\end{tabular}

\subsubsection{St. rubicundus des Tomaros}

Klassische Morphometrie: Die St. rubicundus Männchen des Tomaros haben eine durchschnittliche Vorderflügeloberfläche von $66,06 \mathrm{~mm}^{2}$ (Tab. 6), sind also um 2,89 $\mathrm{mm}^{2}$ kleiner als die der Männchen der allopatrischen St. rubicundus Populationen Griechenlands $\left(68,95 \mathrm{~mm}^{2}\right)$. Mit einer durchschnittlichen Femurlänge von 12,04 mm ist der Femur der St. rubicundus des Tomaros ähnlich lang wie der der griechischen Populationen (11,96 mm). Dementsprechend ist das Verhältnis von Vorderflügeloberfläche zu Femurlänge um 5,11 $\%$ kleiner als bei den St. rubicundus der griechischen Populationen (5,77). Mit anderen 
Worten: Die St. rubicundus Männchen unterscheiden sich hinsichtlich dieser Parameter signifikant von den griechischen St. rubicundus $(\mathrm{p}=0,011)$.

Tab. 6 Durchschnittliche Größe des Vorderflügels und Femurlänge der Männchen und Weibchen verschiedener Populationen auf dem Tomarosmassiv

\begin{tabular}{|c|c|c|c|c|c|c|c|}
\hline Population & $\mathbf{n}$ & $\begin{array}{c}\text { Flächeninhalt } \\
\mathrm{mm}^{2}\end{array}$ & $\boldsymbol{\sigma}$ & $\begin{array}{c}\text { Femurlänge } \\
\text { mm }\end{array}$ & $\boldsymbol{\sigma}$ & $\begin{array}{c}\text { Flächeninhalt/ } \\
\text { Femurlänge }\end{array}$ & $\boldsymbol{\sigma}$ \\
\hline St. rubicundus (Südtomaros) & 23 & 66,06 & 4,95 & 12,04 & 0,59 & 5,49 & 0,38 \\
\hline St. clavatus (Südtomaros) $\widehat{ }$ & 42 & 35,23 & 3,12 & 11,08 & 0,45 & 3,18 & 0,23 \\
\hline St. clavatus (Nordtomaros) ${ }^{\lambda}$ & 17 & 30,18 & 2,94 & 10,85 & 0,52 & 2,78 & 0,21 \\
\hline Individuen Sattel (Südtomaros) ${ }^{\lambda}$ & 80 & 48,62 & 9,00 & 11,51 & 0,41 & 4,21 & 0,72 \\
\hline St. rubicundus (Südtomaros) & 17 & 47,71 & 2,05 & 14,98 & 0,48 & 3,19 & 0,15 \\
\hline St. clavatus (Südtomaros) $q$ & 24 & 33,15 & 3,92 & 14,16 & 0,71 & 2,34 & 0,24 \\
\hline St. clavatus (Nordtomaros) $q$ & 16 & 33,07 & 3,66 & 13,96 & 0,73 & 2,37 & 0,22 \\
\hline Individuen Sattel (Südtomaros) $q$ & 10 & 38,18 & 3,34 & 14,22 & 0,69 & 2,68 & 0,16 \\
\hline
\end{tabular}

St. rubicundus Weibchen des Tomaros haben mit einem Vorderflügelflächeninhalt von $47,71 \mathrm{~mm}^{2}$ und einen um 2,89 $\mathrm{mm}^{2}$ etwas kleineren Flächeninhalt als die St. rubicundus Weibchen der griechischen Populationen $\left(49,72 \mathrm{~mm}^{2}\right)$. Die Femurlänge ist aber mit durchschnittlich 14,98 mm um 0,84 mm größer als die der St. rubicundus-Weibchen der griechischen Populationen $(14,14 \mathrm{~mm})$. Dementsprechend ist das Verhältnis von Flächeninhalt zur Femurlänge mit 3,19 kleiner als bei den St. rubicundus-Weibchen der griechischen Populationen. Wie im Falle der Männchen, so unterscheiden sich auch bei den Weibchen beide Populationen signifikant voneinander $(\mathrm{p}=0,002)$.

\subsubsection{St. clavatus Tomaros}

Klassische Morphometrie: Die St. clavatus-Männchen des Südtomaros weisen eine durchschnittliche Vorderflügeloberfläche von $35,23 \mathrm{~mm}^{2}$ (Tab. 6) auf. Die Vorderflügel sind somit um 4,62 $\mathrm{mm}^{2}$ größer als die der St. clavatus-Population des Xerovouni. Hingegen gibt es nur einen geringen Unterschied zwischen den Individuen des Nordtomaros $\left(30,18 \mathrm{~mm}^{2}\right)$ und des Xerovouni $\left(30,61 \mathrm{~mm}^{2}\right)$. In der Länge der Femora sind ebenfalls nur sehr geringe Unterschiede zwischen den Individuen der beiden St. clavatus-Populationen des Tomaros und der des Xerovouni zu finden. Der Quotient aus Flächeninhalt des Vorderflügels und Femurlänge ergibt bei den St. clavatus des Südtomaros einen Wert von 3,17 und ist somit größer als das Flächeninhalt/Femur-Verhältnis bei Tieren der St. clavatusPopulation des Xerovouni (2,78). Die Individuen des Nordtomaros haben mit 2,87 das gleiche Flächeninhalt/Femur-Verhältnis wie die des Xerovouni. Die Individuen des Nord- 
tomaros haben im Bezug zu ihrer Femurlänge daher einen etwas größeren Vorderflügel. Somit unterscheiden sich die Individuen des Südtomaros bezüglich dieser Merkmale signifikant $(\mathrm{p}<0,001)$ von den Individuen des Nordtomaros und des Xerovouni.

Der Flächeninhalt des Vorderflügels der St. clavatus-Weibchen des Südtomaros ist mit $33,15 \mathrm{~mm}^{2}$ um 1,30 $\mathrm{mm}^{2}$ kleiner als der der weiblichen Individuen auf dem Xerovouni. Der Flächeninhalt der St. clavatus-Weibchen des Nord-Tomaros ist mit 33,07 $\mathrm{mm}^{2}$ ähnlich groß wie bei den St. clavatus-Weibchen des Südtomaros (Tab. 6). Auch im Vergleich der Femurlängen gibt es zwischen den Individuen des Südtomaros (Femurlänge 14,16 mm) und denen des Nordtomaros (Femurlänge 13,95 mm) nur sehr geringe Unterschiede. Aber beide Populationen besitzen einen im Mittel bzw. 0,18 mm kürzeren Femur als die St. clavatus Weibchen des Xerovouni (14,59). Bildet man aber das Verhältnis zwischen Flächeninhalt des Vorderflügels und der Femurlänge, so relativieren sich die zuvor beschrieben Unterschiede. Die Werte aller drei Populationen liegen in etwa gleich (siehe Tabelle). Die Weibchen der St. clavatus-Populationen unterscheiden sich somit nicht signifikant voneinander.

\subsubsection{Individuen des Sattels auf dem Südtomaros}

Vergleicht man den durchschnittlichen Flächeninhalt des Vorderflügels der Individuen (Tab. 6) des Sattels zwischen Süd- und Nordhang des Südtomaros mit denen der Referenzgruppen so sind diese mit durchschnittlich mit 48,61 $\mathrm{mm}^{2}$ um $20,34 \mathrm{~mm}^{2}$ kleiner als die der Art St. rubicundus und um 18,00 $\mathrm{mm}^{2}$ größer als die Vorderflügel der Art St. clavatus $\left(30,61 \mathrm{~mm}^{2}\right)$. Der Femur der Individuen der Kontaktzone ist mit durchschnittlich 11,51 mm um 0,45 mm kürzer als der der St. rubicundus der griechischen Populationen. Im Vergleich mit St. clavatus hingegen weisen die Individuen der Kontaktzone I einen um 0,49 mm längeren Femur auf. Das Verhältnis von Flügelflächeninhalt und Femurlänge beträgt bei den Individuen der Kontaktzone bei 4,21 und liegt somit zwischen den errechneten Werten der allopatrischen Populationen der Arten St. clavatus $(2,78)$ und St. rubicundus $(5,77)$. Der Scheffé-Test zeigt signifikante Unterschiede zwischen der Population des Sattels und der Arten St. clavatus und St. rubicundus.

Der Flächeninhalt des Vorderflügels der Weibchen des Sattels ist mit durchschnittlich $38,18 \mathrm{~mm}^{2}$ um 11,43 $\mathrm{mm}^{2}$ kleiner als der der St. rubicundus-Populationen Griechenlands. Im Vergleich zur St. clavatus-Population des Xerovouni ist der Flächeninhalt um 3,74 m² 
kleiner. Die Femurlänge der Weibchen der Kontaktzone liegt mit durchschnittlich 14,22 $\mathrm{mm}$ im Wertebereich von St. rubicundus $(14,14 \mathrm{~mm})$ und St. clavatus $(14,59 \mathrm{~mm})$. Hingegen ist das Verhältnis von Vorderflügeloberfläche und Femurlänge der Weibchen der Kontaktzone mit 2,68 kleiner als das der St. rubicundus-Weibchen und etwas größer als das durchschnittliche Verhältnis bei den St. clavatus-Weibchen des Xerovouni. Die Weibchen vom Sattel des Südtomaros unterscheiden sich somit, wie auch die Männchen, signifikant von den Individuen der Arten St. rubicundus und St. clavatus.

\subsubsection{Betrachtung der einzelnen Individuen}

In Abb. 39 werden nun für die Männchen der einzelnen Populationen der Flächeninhalt des Vorderflügels gegen die Femurlänge aufgetragen. Es wird deutlich, dass die Tiere der Art St. clavatus einen kürzeren Femur und auch Flügel mit kleinerem Flächeninhalt haben als die Individuen der Art St. rubicundus. St. clavatus grenzt sich stark von den St. rubicundus-Populationen ab. Zwischen diesen beiden Arten verteilen sich die Individuen der Kontaktzonen. Die Individuen der St. rubicundus-Population des Tomaros unterscheiden sich etwas von denen der allopatrischen Populationen Griechenlands.

Die St. clavatus Population des Tomaros grenzt sich leicht von der Xerovouni-Population ab. Das heißt, dass die Individuen des Xerovouni und des Südtomaros sich leicht unterscheiden. Die Tiere des Nordtomaros zeigen große Ähnlichkeit zur Xerovouni-Population und überlappen sich deshalb in Abb. 39. Leichte Unterschiede sind zwischen den Populationen des Nord- und Südtomaros zu sehen. 


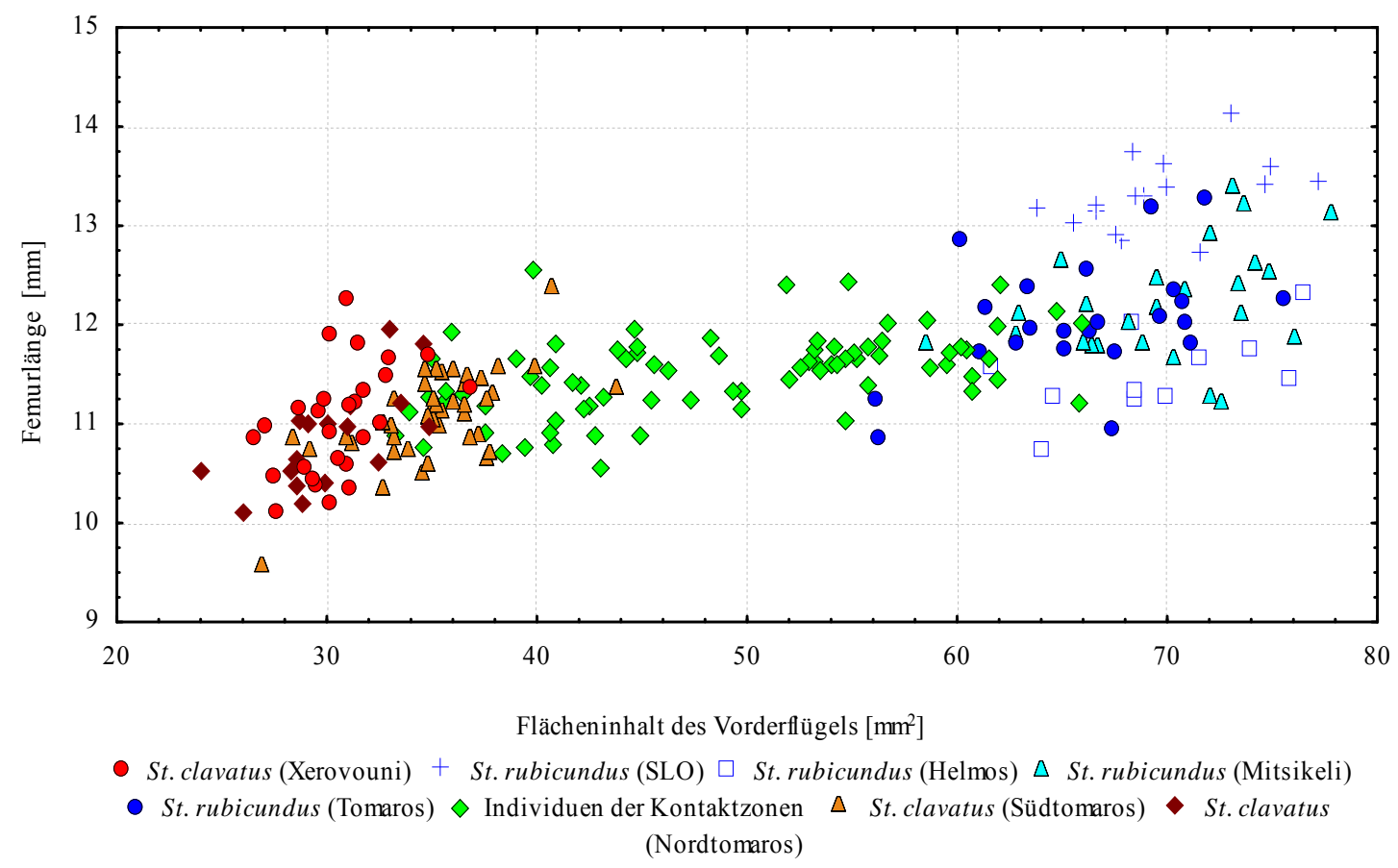

Abb. 39 Flächeninhalt der Vorderflügel in Bezug zur Länge des Hinterfemurs bei Männchen verschiedener Populationen

Bei den Weibchen (Abb. 39) sind die Verhältnisse dagegen nicht so eindeutig wie bei den Männchen. (Abb. 40) Anders als diese unterscheiden sich die Weibchen der Arten St. clavatus und St. rubicundus einzig in dem Flächeninhalt der Vorderflügel, aber nicht eindeutig in der Femurlänge. In Gegensatz zu den Männchen zeigen die St. clavatus des Tomaros in Vergleich zu den des Xerovouni hinsichtlich dieser Parameter keine veränderten morphologischen Eigenschaften. Das heißt, dass sich die Weibchen des Süd- und des Nordtomaros gegenüber denen des Xerovouni nicht unterscheiden. 


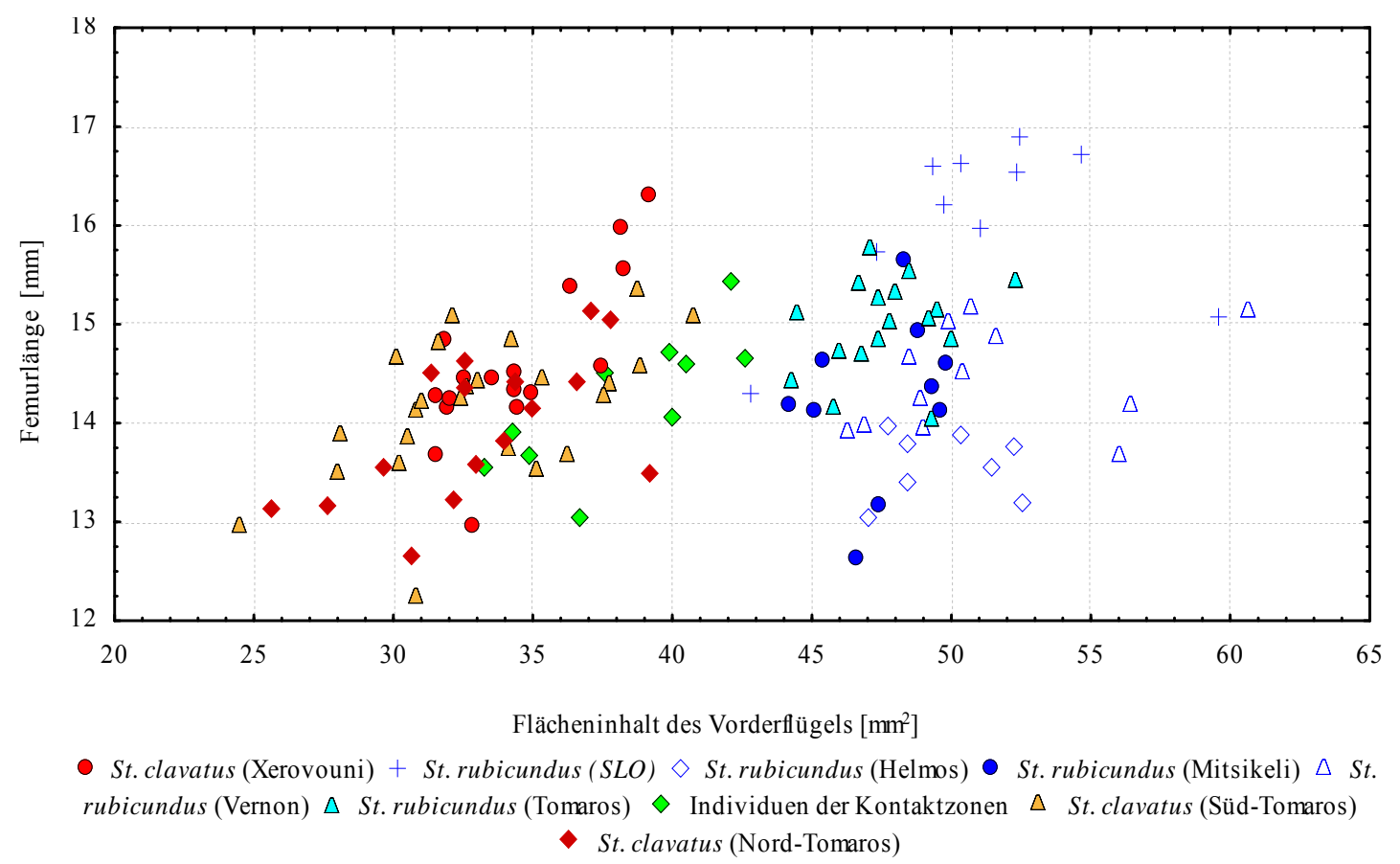

Abb. 40 Flächeninhalt der Vorderflügel in Bezug zur Länge des Hinterfemurs bei Weibchen verschiedener Populationen

\section{Zusammenfassung}

Die klassische Methode eignet sich zur Charakterisierung und Unterscheidung beider Arten. Dabei sind nicht nur signifikante Unterschiede zwischen den beiden Arten St. clavatus und St. rubicundus errechnet worden, sondern auch Signifikanzen zwischen der alpinen und griechischen St. rubicundus Population. Größenunterschiede der Flügel und Femora, aber auch unterschiedliche Flügelstruktur (Form) machen den Unterschied zwischen den Arten aus. Grundsätzlich findet man bei Männchen wie Weibchen trotz des Geschlechtsdimorphismus ähnliche zwischenartliche Unterschiede.

\subsubsection{Geometrische Morphometrie an einzelnen Individuen des Tomaros}

Allgemeine Übersicht: Anders als bei der zuvor untersuchten St. clavatus-Population des Xerovouni und den St. rubicundus-Populationen, die jeweils völlig getrennt von Populationen der jeweils anderen Art sind, grenzen auf dem Tomaros die Verbreitungsgebiete aneinander. Die geographische Verteilung der beiden Arten ist sehr heterogen, und an den Habitatsgrenzen beider Arten findet man intermediäre Individuen in allen Übergangsstufen. Somit ist eine exakte Einteilung auf Populationsebene kaum möglich, da solch eine Einteilung klare Populationsgrenzen voraussetzt, die auf dem Tomaros kaum zu finden sind. 
Es erweist sich als notwendig, die einzelnen, auf dem Tomaros vorkommenden Individuen in Bezug auf die Referenzgruppen morphometrisch einzuordnen und auf Grundlage ihrer Fundorte in Gruppen zusammenzufassen (Kapitel 2.2). Grundlage für die Einordnung der Tomarostiere ist zunächst die exakte Charakterisierung der Referenzgruppen von St. rubicundus und St. clavatus. Jedes Tomaros-Individuum wird mit den Referenzgruppen verglichen, und es wird daraufhin bestimmt, welcher der beiden Arten es zuzuordnen ist bzw. ob es Merkmale beider Arten, ggf. zu welchen Anteilen, besitzt.

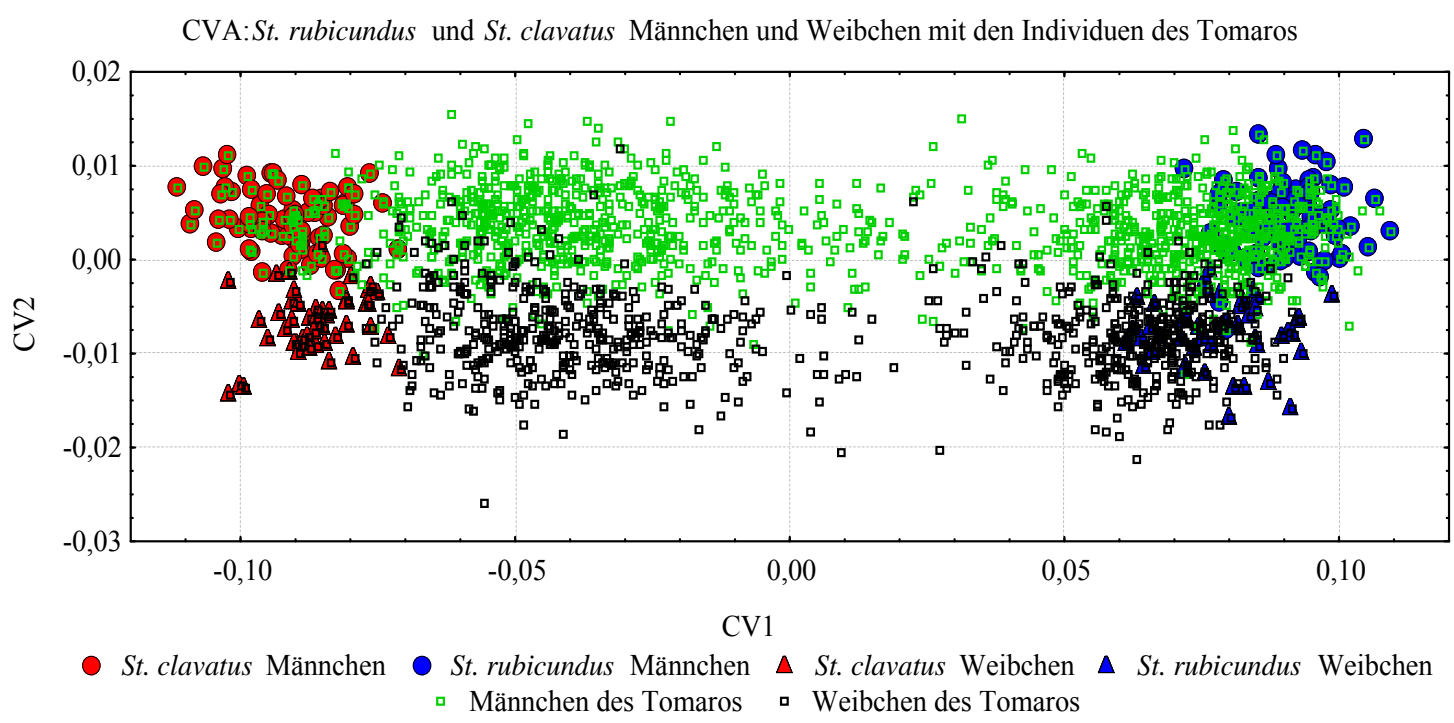

Abb. 41 Kanonische Varianzanalyse der Individuen des Tomaros zusammen mit denen der Referenzgruppen

In Abb. 41 ist zu erkennen, dass sich der Großteil der Tomaros-Individuen in das CVASystem, zu den Männchen und Weibchen der Referenzgruppen einordnen lässt. Auffällig sind hier die beiden sich in großen Teilen voneinander abgrenzenden Cluster zwischen den jeweiligen Männchen und Weibchen der Referenzgruppen. Zwischen den Männchen und Weibchen der Referenzpopulationen ordnen sich die Individuen des Tomaros an. Diese bilden vier Cluster die größten teils zwischen den Referenzpopulationen liege, sich aber auch an einigen Stellen mit diesen überlappen.

\subsubsection{Einordnung der Individuen des Tomaros beider Referenzgruppen}

Die Einordnung jedes einzelnen Individuums der genannten Populationen (siehe Abb. 41) findet auf Basis der kanonischen Varianzanalyse der beiden Referenzgruppen statt. Hier wird nun jedes Individuum mit den Referenzgruppen beider Arten verglichen. So kann 
exakt bestimmt werden, ob ein Individuum Merkmale einer der beiden Arten oder ob es Merkmale beider Arten besitzt.

Hierzu dient die Bestimmung der von Nolte und Sheets (2005) verwendeten MahalanobisDistanz (Kapitel 2.4.7). Dieses Verfahren berechnet die Distanz jedes Individuums zum Mittelpunkt der jeweiligen Referenzgruppe in der kanonischen Varianzanalysenmatrix. Diese Distanzmessung bezieht aber auch die Skalierung der Variationsebenen mit ein (Näheres in Material und Methoden Kapitel 2.4.8). Es wird also berechnet, wie ähnlich die Morphologie des Vorderflügels jedes einzelnen Individuums zu der Referenzgruppe von St. rubicundus und der Referenzgruppe von St. clavatus ist. Somit lässt sich jedes Individuum aufgrund der Differenz der Mahalanobis-Distanzen eindeutig charakterisieren.

Es wird also die Mahalanobis-Distanz von jedem Individuum einmal zur St. clavatusReferenzgruppe und einmal zur St. rubicundus-Referenzgruppe bestimmt, d. h. es werden immer zwei Werte pro Tier ermittelt. Wenn ein Individuum auf Basis der Vorderflügelmorphologie sehr viele Gemeinsamkeiten mit St. clavatus hat, hat es eine kleine Mahalanobis-Distanz zum Mittelpunkt der St. clavatus-Referenzgruppe. Dementsprechend hat dieses Individuum eine große Mahalanobis-Distanz zum Mittelpunkt der St. rubicundusReferenzgruppe. Dies ergibt sich daraus, dass einzig die Varianz zwischen diesen beiden Referenzgruppen die Basis für diese morphologische Einordnung ist. Die Summe beider Mahalanobis-Distanzen ergibt so immer den gleichen Wert. Um dieses System etwas zu vereinfachen, wird nun die Differenz zwischen den beiden Werten, die sogenannte Mahalanobis-Distanz-Differenz, errechnet.

Da St. clavatus in der Reihenfolge der Gruppen bei der kanonischen Varianzanalyse die Stelle 1 zugeordnet wurde, berechnet das Programm zunächst die Distanz jedes Individuums zu dem Mittelpunkt der St. clavatus Population. Ein Individuum, das hauptsächlich St. clavatus ähnelt, hat folglich eine kleine Mahalanobis-Distanz zum Mittelpunkt der St. clavatus-Referenzgruppe und eine dementsprechend große Mahalanobis-Distanz zum Mittelpunkt der St. rubicundus-Referenzgruppe. Also ergibt sich eine Mahalanobis-DistanzDifferenz im hohen negativen Bereich (Tab. 7). Der umgekehrte Fall ist bei einem Individuum mit hauptsächlichen St. rubicundus-Merkmalen zu beobachten. Dieses Tier hat eine große Mahalanobis-Distanz zur St. clavatus-Referenzgruppe und eine kleine MahalanobisDistanz zur St. rubicundus-Referenzgruppe. Die Differenz beider Werte ergibt einen hohen 
positiven Wert. Somit kann ebenfalls die Varianz innerhalb der Referenzgruppen ermittelt werden, welche nun die Mahalanobis-Distanz jedes Individuums zum Mittelpunkt seiner Referenzgruppe darstellt.

Tab. 7 Flügelmorphometrische Berechnungen für einzelner Individuen der St. clavatus- und St. rubicundus- Referenzgruppen. Durch Berechnung der Mahalanobis (M)-Distanz

\begin{tabular}{|c|c|c|c|c|c|c|c|}
\hline ID & GPS & Jahr & Population & SEX & $\begin{array}{l}\text { M-Distanz } \\
\text { St. clavatus } \\
\end{array}$ & \begin{tabular}{c|} 
M-Distanz \\
St. rubicundus \\
\end{tabular} & M-Differenz \\
\hline 214 & $\mathrm{X} 5$ & 08 & Xerovouni & $\hat{0}$ & 2,055063 & 21,63774 & $-19,5827$ \\
\hline 1011 & $\mathrm{X} 5$ & 09 & Xerovouni & 0 & 1,012689 & 22,68011 & $-21,6674$ \\
\hline 090 & $\mathrm{X} 3$ & 07 & Xerovouni & $0^{\lambda}$ & 0,123206 & 23,5696 & $-23,4464$ \\
\hline 091 & $\mathrm{X} 3$ & 07 & Xerovouni & $\hat{0}$ & 0,226258 & 23,46655 & $-23,2403$ \\
\hline 804 & $\mathrm{Mi} 3$ & 09 & Mitsikeli & $\hat{0}$ & 21,63157 & 2,06123 & 19,57034 \\
\hline 382 & $\mathrm{MiA}$ & 08 & Mitsikeli & $\hat{0}$ & 23,13951 & 0,553292 & 22,58622 \\
\hline 383 & $\mathrm{MiA}$ & 08 & Mitsikeli & 0 & 22,706 & 0,986807 & 21,71919 \\
\hline 359 & $\mathrm{MiB}$ & 08 & Mitsikeli & $\sigma^{\lambda}$ & 22,77434 & 0,918464 & 21,85588 \\
\hline
\end{tabular}

Für die 159 männliche Tiere (78 St. clavatus und 81 St. rubicundus) der beiden ReferenzGruppen ergeben sich folgende Mahalanobis-Distanzwerte zu den jeweiligen Mittelpunkten der Referenzgruppen: Bei den Individuen von St. clavatus, die allesamt aus der Xerovouni-Population stammen, zeigen sich Distanzwerte zum Mittelpunkt der St. clavatusReferenzgruppe zwischen 0,009 und 2,41. Erwartungsgemäß wesentlich größer sind die Distanzwerte von diesen Individuen zu St. rubicundus; sie liegen hier zwischen 21,5 und 26,1. St. rubicundus zeigt innerartliche Distanzwerte zwischen 0,024 und 2,477 sowie zwischenartliche Distanzwerte von 21,51 bis 26,14 zu St. clavatus. Beide Arten haben innerartliche Varianzen $(\operatorname{Var}(\mathrm{x})=0,366)$ für St. clavatus und $\operatorname{Var}(\mathrm{x})=0,317$ für St. rubicundus), die sich nicht signifikant unterscheiden.

Die Differenz aus innerartlicher und zwischenartlicher Distanz dient der Einordnung, zu welcher Art die Individuen gehören. Wie bereits erwähnt, errechnet CVAgen6 zuerst die Distanz zu St. clavatus und danach die Distanz zu St. rubicundus. Daher ergibt die Differenz beider Werte für Individuen von St. clavatus einen negativen Wert von -23,69 bis 19,38 und für St. rubicundus einen positiven Wert von 19,33 bis 23,69.

Auch für die Einordnung der Weibchen wurden die Vorderflügel der beiden Referenzgruppen morphometrisch vermessen. Die Referenzgruppen, bestanden aus 48 St. clavatus Weibchen aus der Xerovouni-Population und 50 St. rubicundus Weibchen aus den griechischen Populationen. 
Die Weibchen zeigten mit einer intraspezifischen Mahalanobis-Distanz zwischen 0,014 und 2,287 bei St. clavatus und mit Werten zwischen 0,018 und 1,987 bei St. rubicundus ähnliche Werte wie die Männchen. Einzig die interspezifische Distanz, die bei St. clavatus zwischen 17,73 und 21,24 liegt, und bei St. rubicundus zwischen 17,60 und 21,24, ist im Vergleich zu den Männchen etwas kleiner.

Bei den Weibchen werden Individuen mit einer Mahalanobis-Distanz-Differenz von -17,6 bis -21,24 der Art St. clavatus zugeordnet. Der Art St. rubicundus entsprechen Weibchen mit einer Mahalanobis-Distanz-Differenz von 17,6 bis 21,24.

Fazit: Mit der genannten Methode kann nunmehr für jedes auf dem Tomaros gefundene Tier, gleich ob Männchen oder Weibchen, eine Mahalanobis-Distanz-Differenz ermittelt werden, und es ist somit möglich, auch intermediäre Individuen exakt einzuordnen. Hierbei gilt aufgrund der für die Referenzgruppen ermittelten Werte, dass Männchen, die eine Mahalanobis-Distanz-Differenz von -23,69 bis -19,38 besitzen, die gleiche Vorderflügelmorphologie wie die Tiere der St. clavatus-Referenzgruppe haben, also ohne Einschränkung dieser Art zugeordnet werden können. Entsprechend gilt, dass Individuen mit einer Mahalanobis-Distanz-Differenz von 19,33 bis 23,69 eine Vorderflügelmorphologie besitzen, die der St. rubicundus-Referenzgruppe entspricht. Bei intermediären Individuen wäre eine Mahalanobis-Distanz-Differenz um Null charakteristisch für Tiere mit gleichen Anteilen an Merkmalen von St. rubicundus und St. clavatus.

\subsubsection{Merkmalsanteile und Hybridindex}

Für eine übersichtliche, aber immer noch hinreichend detaillierte Charakterisierung der auf dem Tomaros vorkommenden St. rubicundus und St. clavatus bzw. deren Übergangsformen zuzurechnenden Individuen, hat sich folgende, auf der Mahalanobis-DistanzDifferenz basierende Einteilung als zweckmäßig erwiesen:

Hierzu wurde bei jedem Tier bestimmt, zu wie viel Prozent es auf der Grundlage der Vorderflügelmorphologie einer der beiden Arten entspricht (Kapitel 2.4). Demnach haben Individuen innerhalb des Varianzbereiches der St. clavatus-Referenzgruppe (MahalanobisDistanz-Differenz zwischen -23,69 und -19,38) einen Anteil von 100\% St. clavatus und $0 \%$ St. rubicundus. Bei den St. rubicundus der Referenzgruppe ist dies umgekehrt. Ein intermediäres Tier mit einer Mahalanobis-Distanz-Differenz von 5,9 würde zu 65 \% St. rubicundus und zu 35 \% St. clavatus entsprechen. Da sich beide Prozentwerte immer zu 
100\% ergänzen, reicht einer der beiden Werte - St. rubicundus-Anteil oder St. clavatusAnteil - aus, um ein Individuum exakt zu charakterisieren. In der vorliegenden Arbeit werden, ausgehend von St. rubicundus, die Tiere wie folgt eingeteilt: Wenn ein Individuum einen Merkmalsanteil von 0\% hat, handelt es sich um ein Tier, das der St. clavatusReferenzgruppe entspricht. Entspricht ein Tier der St. rubicundus-Referenzgruppe, hat es einen Merkmalsanteil von $100 \%$. Intermediäre Individuen sind durch prozentuale Werte von $0 \%<\mathrm{x}<100 \%$ charakterisiert. So bezeichnet beispielsweise ein Wert von $45 \%$ ein Tier mit einem St. rubicundus-Merkmalsanteil von 45\%. und einem St. clavatusMerkmalsanteil von 55\% .

Für eine etwas gröbere Übersicht wird jedem Individuum, getrennt nach Geschlecht, auf Grundlage der individuellen Morphologie zusätzlich ein Hybridindex zugeteilt, wobei eine zwölfstufige Skala angewendet wird. Index 1 steht hier für Tiere, die morphologisch der St. clavatus-Referenzpopulation des Xerovouni entsprechen; Index 12 für jene, die der griechischen St. rubicundus-Referenzpopulation entsprechen. Index 2 bis 11 sind somit Indices, die intermediäre Individuen zwischen den beiden Referenzpopulationen charakterisieren. Das Hybridindexsystem teilt die Individuen in Klassen ein und erleichtert somit die Beschreibung der Merkmalsverteilung in den Populationen.

Tab. 8 Einteilung der Merkmalsanteile und der Hybridindices (HI) auf Basis der MahalanobisDistanz-Differenz (MDD) bei den Männchen.

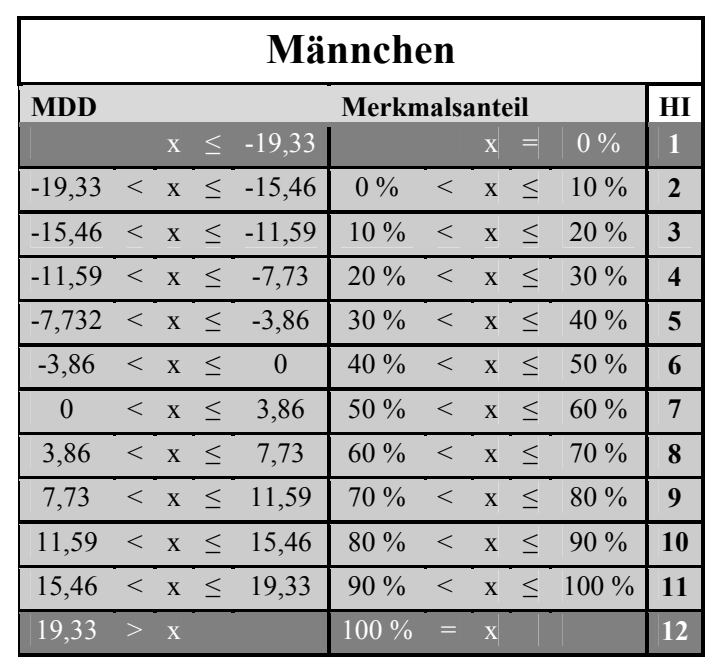

Tab. 9 Einteilung der Merkmalsanteile und der Hybridindices (HI) auf Basis der MahalanobisDistanz-Differenz (MDD) bei den Weibchen.

\begin{tabular}{|c|c|c|c|c|c|c|c|c|c|}
\hline \multicolumn{10}{|c|}{ Weibchen } \\
\hline \multirow{2}{*}{ MDD } & & \multicolumn{7}{|c|}{ Merkmalsanteil } & \multirow{2}{*}{$\begin{array}{c}\text { HI } \\
1\end{array}$} \\
\hline & & $\mathrm{x}$ & $\leq$ & $-17,6$ & & & & $=0 \%$ & \\
\hline$-17,6$ & $<$ & $\mathrm{x}$ & $\leq$ & $-14,08$ & $0 \%$ & $<$ & $\mathrm{x} \leq$ & $\leq 10 \%$ & 2 \\
\hline$-14,08$ & $<$ & $x$ & $\leq$ & $-10,56$ & $10 \%$ & $<$ & $x \leq$ & $\leq 20 \%$ & 3 \\
\hline$-10,56$ & $<$ & $\mathrm{x}$ & $\leq$ & $-7,04$ & $20 \%$ & $<$ & $x \leq$ & $\leq 30 \%$ & 4 \\
\hline$-7,04$ & $<$ & $\mathrm{x}$ & $\leq$ & $-3,52$ & $30 \%$ & $<$ & $\mathrm{x} \leq$ & $\leq 40 \%$ & 5 \\
\hline$-3,52$ & $<$ & $\mathrm{x}$ & $\leq$ & 0 & $40 \%$ & $<$ & $x \leq$ & $\leq 50 \%$ & 6 \\
\hline 0 & $<$ & $x$ & $\leq$ & 3,52 & $50 \%$ & $<$ & $x \leq$ & $\leq 60 \%$ & 7 \\
\hline 3,52 & $<$ & $\mathrm{x}$ & $\leq$ & 7,04 & $60 \%$ & $<$ & $\mathrm{x} \leq$ & $\leq 70 \%$ & 8 \\
\hline 7,04 & $<$ & $\mathrm{x}$ & $\leq$ & 10,56 & $70 \%$ & $<$ & $\mathrm{x} \leq$ & $\leq 80 \%$ & 9 \\
\hline 10,56 & $<$ & $\mathrm{x}$ & $\leq$ & 14,08 & $80 \%$ & $<$ & $\mathrm{x}$ & $\leq 90 \%$ & 10 \\
\hline 14,08 & $<$ & $\mathrm{x}$ & $\leq$ & 17,6 & $90 \%$ & $<$ & $\mathrm{x}=$ & $\leq 100 \%$ & 11 \\
\hline 17,6 & $>$ & $\mathrm{x}$ & & & $100 \%$ & $=$ & $x$ & & 12 \\
\hline
\end{tabular}

Anschließend wurden die mit der Methode der geometrischen Flügelmorphologie gewonnenen Daten mit den zuvor ermittelten Größen der Vorderflügelfläche/Femurlänge mitein- 
ander in Bezug gesetzt. Die Korrelationsanalyse ergab eine signifikante Korrelation von $\mathrm{R}=0$,94. Relative Flügelgröße und Flügelmorphologie korrelieren also stark miteinander. Somit sind die Veränderungen in der Flügelform eng mit der Veränderung der relativen Vorderflügeloberfläche verbunden.

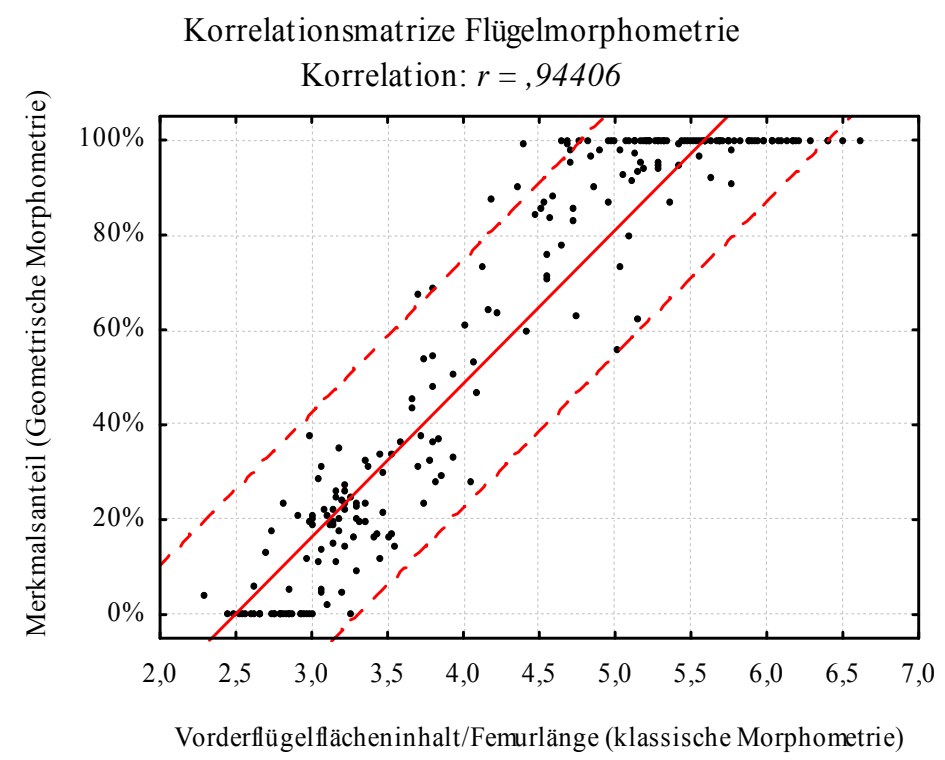

Abb. 42 Korrelationsanalyse der Merkmalsanteile der Vorderflügel- und des Verhältnisses von Vorderflügelflächeninhalt und Femurlänge. Regressionsgerade (-) und Regressionsbänder (- -) (Prognostiziertes Intervall: Niveau 0,95)

\subsubsection{Merkmalsverteilung an den einzelnen Fundorten}

In den Untersuchungsgebieten wurden 154 GPS-Punkte gesetzt, die einen Abstand von mindestens $50 \mathrm{~m}$ voneinander haben. Im Umkreis von ungefähr $20 \mathrm{~m}$ jedes GPS-Punktes wurden Heuschrecken gesammelt und der geometrischen Morphometrie unterzogen. Pro GPS-Punkt wird den dort gefangenen Individuen jeweils ein Hybridindex zugeteilt, und des Weiteren wird der mittlere Merkmalsanteil berechnet.

\subsubsection{Griechische St. rubicundus Populationen außerhalb des Tomaros-Gebirges}

Bevor die Individuen des Tomaros beschrieben werden, werden die Individuen aus den Standorden an denen nur eine der beiden Arten gefunden wurden, untersucht.

Der Tab. 10 ist zu entnehmen, dass in allen Populationen der Großteil der Individuen einen Hybridindex von zwölf hat. Nur einige wenige Tiere haben Index 11 oder 10. Bei der Be- 
trachtung der Merkmalsanteile zeigt sich, dass die Populationen im Mittel einen St. rubicundus Anteil von 99,6-99,9 \% bei einer Standardabweichung von 0,001 bis 2,32 haben. Wie auch bei den St. rubicundus Populationen wurden die auf dem Xerovouni gesammelten Individuen mit den Referenzpopulationen verglichen. Der Großteil dieser Tiere entspricht der St. clavatus Referenzgruppe. Einzig 13 Tiere sind dem Hybridindex 2 und eines dem Hybridindex 3 zuzuordnen. Die Merkmalsanteile liegen durchschnittlich bei 0,22 Prozent bei einer Standardabweichung von 1,54.

Tab. 10 Merkmalsverteilung der St. rubicundus (blau) und St. clavatus- Referenzpopulationen (rot)

\begin{tabular}{|l|c|c|c|c|c|c|c|c|c|c|c|c|c|c|c|}
\hline Population & \multicolumn{10}{|c|}{ Hybridindices } & \multicolumn{1}{|c|}{ Merkmalsanteil } \\
\cline { 2 - 16 } & 1 & 2 & 3 & 4 & 5 & 6 & 7 & 8 & 9 & 10 & 11 & 12 & $\mathbf{\Sigma}$ & $\overline{\mathbf{x}}$ & $\sigma$ \\
\hline Mitsikeli & - & - & - & - & - & - & - & - & - & 2 & 8 & 143 & $\mathbf{1 5 3}$ & $\mathbf{9 9 , 8 4 \%}$ & 2,32 \\
\hline Vernon & - & - & - & - & - & - & - & - & - & - & 1 & 67 & $\mathbf{6 8}$ & $\mathbf{9 9 , 9 9 \%}$ & 0,001 \\
\hline Helmos & - & - & - & - & - & - & - & - & - & - & 1 & 29 & $\mathbf{3 0}$ & $\mathbf{9 9 , 9 8 \%}$ & 0,01 \\
\hline Valtou & - & - & - & - & - & - & - & - & - & - & 1 & 20 & $\mathbf{2 1}$ & $\mathbf{9 9 , 9 8 \%}$ & 0,01 \\
\hline Metsovo & - & - & - & - & - & - & - & - & - & - & 7 & 42 & $\mathbf{4 9}$ & $\mathbf{9 9 , 5 9 \%}$ & 2,02 \\
\hline Xerovouni & 245 & 13 & 1 & - & - & - & - & - & - & - & - & - & $\mathbf{2 5 9}$ & $\mathbf{0 , 2 2 \%}$ & 1,54 \\
\hline
\end{tabular}

\subsubsection{Charakterisierung der Tomaros-Populationen von St. clavatus, St. rubicun- dus und deren Hybriden aufgrund der Vorderflügelmorphologie}

Allgemeine Übersicht: Für die morphometrische Vermessung der Vorderflügel wurden auf dem Tomaros-Gebirge an 132 Standorten (GPS-Punkten; siehe Tab. 11 bis Tab. 17 mit geographischen Koordinaten und Höhenangaben) insgesamt 2691 (1612 ふં; 1079 †) Individuen der Arten St. clavatus und St. rubicundus sowie deren Zwischenformen herangezogen.

Es fällt sofort auf, dass - anders als auf den übrigen Gebirgen - hier jede der zwölf Kategorien des Hybridindexsystems vertreten ist (

Abb. 43): 538 (19,99\%) der Tiere können dem Index 12 zugeordnet werden, entsprechen also der Referenz-Gruppe von St. rubicundus. Demgegenüber sind nur 96 Individuen $(3,57 \%)$ mit dem Index $1 \mathrm{zu}$ charakterisieren, d. h. als reine St. clavatus anzusprechen. Stärker vertreten sind Heuschrecken, die zwar noch überwiegend St. clavatus ähneln, aber doch hinsichtlich der Flügelmorphologie bereits deutliche St. rubicundus-Merkmale zeigen. Dies drückt sich in der Zuordnung zu Index 2 (246 Tiere = 9,14\%), Index 3, (409 Tiere $=15,20 \%)$, Index $4(335$ Tiere $=12,45 \%)$ und Index $5(209$ Tiere $=7,77 \%)$ aus. Tiere mit den Hybridindices 6 - 10 sind weniger stark vertreten: Index $6(132$ Tiere $=4,90 \%)$; 
Index $7(69$ Tiere $=2,56 \%)$; Index $8(53$ Tiere $=1,97 \%)$; Index $9(86$ Tiere $=3,20 \%)$; Index 10 (162 Tiere =6,02\%). Häufiger sind dann erst wieder Tiere mit dem Index 11 (353 Tiere 13,12\%), die hinsichtlich der Flügelmerkmale bereits sehr stark St. rubicundus ähneln.

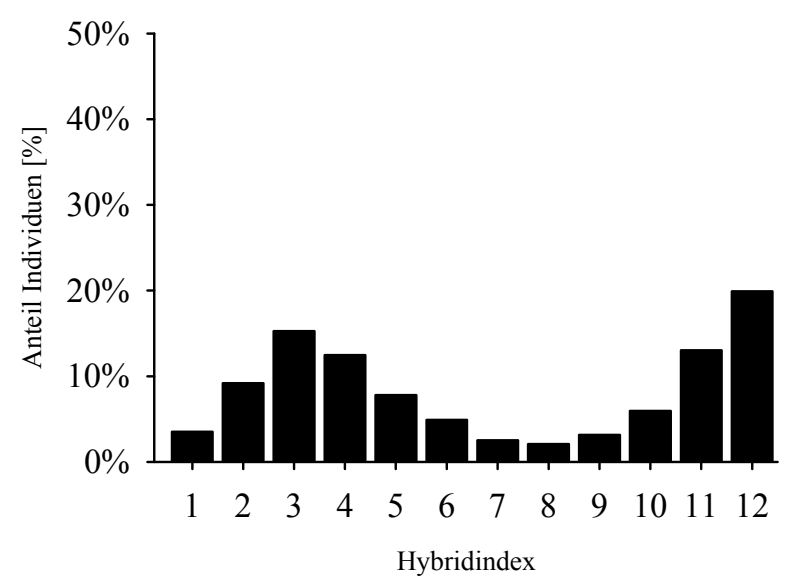

Abb. 43 Gesamtverteilung der Hybridindices (Vorderflügelmerkmal) auf dem gesamten Tomaros-Massiv.

Im Folgenden werden die an den einzelnen GPS-Punkten zu findenden Teilpopulationen beschrieben, sowie in Tabellenform und Grafiken dokumentiert. Hierzu wird aus praktischen Gründen das Tomarosgebirge in drei Gebiete eingeteilt (siehe Abb. 44). Das erste Gebiet umfasst die gesamte Hochfläche des Nord-Massivs. Hier wurden insgesamt 29 GPS-Punkte gesetzt und 475 (250 ふ̊; 225 ㅇ) Tiere flügelmorphometrisch vermessen. Das Süd-Massiv wird in zwei Zonen eingeteilt, nämlich zum einen den Osthang und zum anderen den Süd- und Nordhang. Auf dem Osthang wurden 697 Tiere (377 §̂; 320 ๆ) gefangen und vermessen, die sich auf 39 GPS-Punkte verteilen. Im dritten Untersuchungsgebiet, dem Süd- und Nordhang des Süd-Massivs, wurden 69 GPS-Punkte gesetzt und insgesamt 1519 Tiere (985 §’; 534 ㅇ) untersucht. Es sei noch einmal betont, dass die Unterteilung in die drei genannten Gebiete allein deshalb erfolgt, um die folgende Beschreibung in sinnvoller Weise zu gliedern. Die Gebiete sind nicht isoliert voneinander, und deshalb folgt am Schluss auch eine zusammenfassende Betrachtung. 


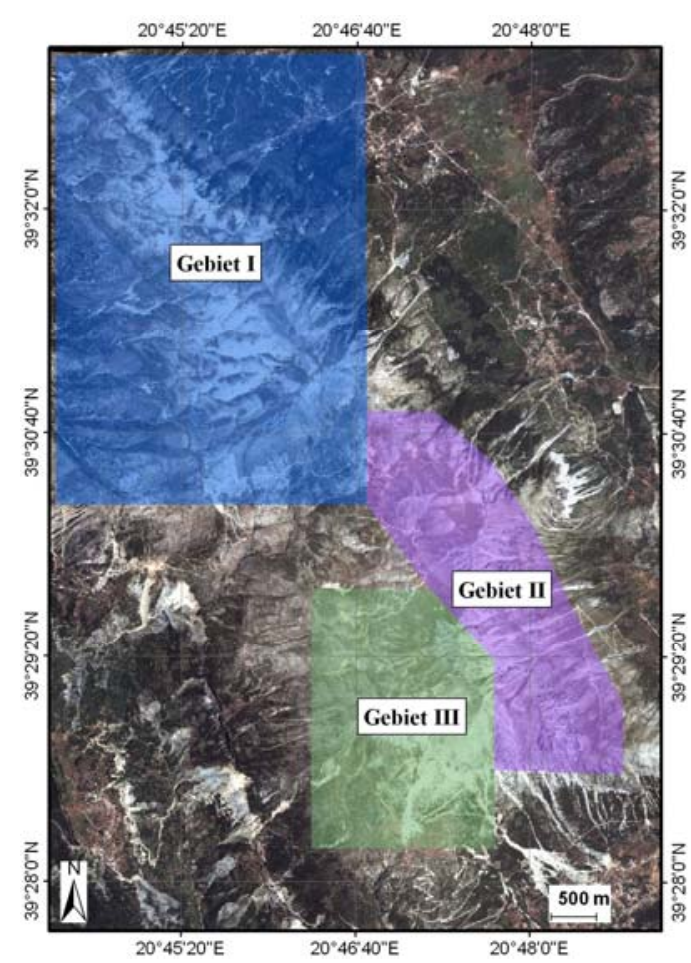

Abb. 44 Einteilung des Tomaros Gebirges in drei Teilgebiete (I-III).

Die Einzeldaten aller untersuchten 2691 Tiere sind auf der beigegebenen CD abgelegt. Der besseren Anschaulichkeit halber sind zudem die Verhältnisse in zweifacher Weise graphisch dargestellt: (1) Für jeden GPS-Punkt sind die St. clavatus (rot) bzw. St. rubicundus (blau) betreffenden Merkmalsanteile in einem Kreisdiagramm gezeigt. (2) Da diese summarische Darstellung nichts über die individuelle Zusammensetzung der Population an dem betreffenden Punkt aussagt, wird diese in einer zweiten Grafik in Form von Säulen illustriert. Deren farbige Abschnitte (zum Farbcode siehe Abb. 47) geben für jeden der GPS-Punkte die prozentualen Anteile der verschiedenen Zwischenformen an. Die genauen Zahlen sind der genannten Tabelle zu entnehmen.

\subsubsection{Merkmalsverteilung auf dem Tomaros-Nordmassiv (Gebiet I)}

Allgemeine Übersicht: Auf der Hochfläche des Nordtomaros sind vor allem Tiere zu finden, die entweder völlig der Xerovouni-Referenzpopulation von St. clavatus entsprechen oder dieser doch noch sehr stark ähneln (Abb. 45). So sind von den 475 für die Vorderflügelmorphometrie herangezogenen Tieren 84 Individuen (17,6\%) dem Index 1 (St. clavatus), 145 Tiere (30,5\%) dem Index 2 und 128 Tiere (26,9\%) dem Index 3 zuzuordnen. Weit weniger Tiere gleichen der St. rubicundus-Referenzgruppe: Mit dem Index 12 sind 36 In- 
dividuen (7,6\%) und solche mit Index 1119 Tiere (4,0\%) zu charakterisieren. Tiere mit Hybridindex 5 bis 10 sind nur mit $0,6 \%$ bis $1,7 \%$ vertreten.

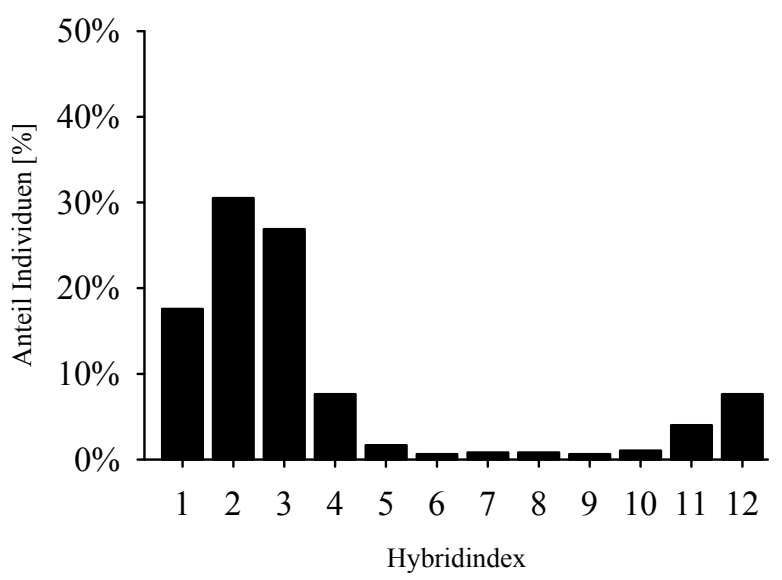

Abb. 45 Gesamtverteilung der Hybridindices (Vorderflügelmerkmal) auf dem Nordtomaros.

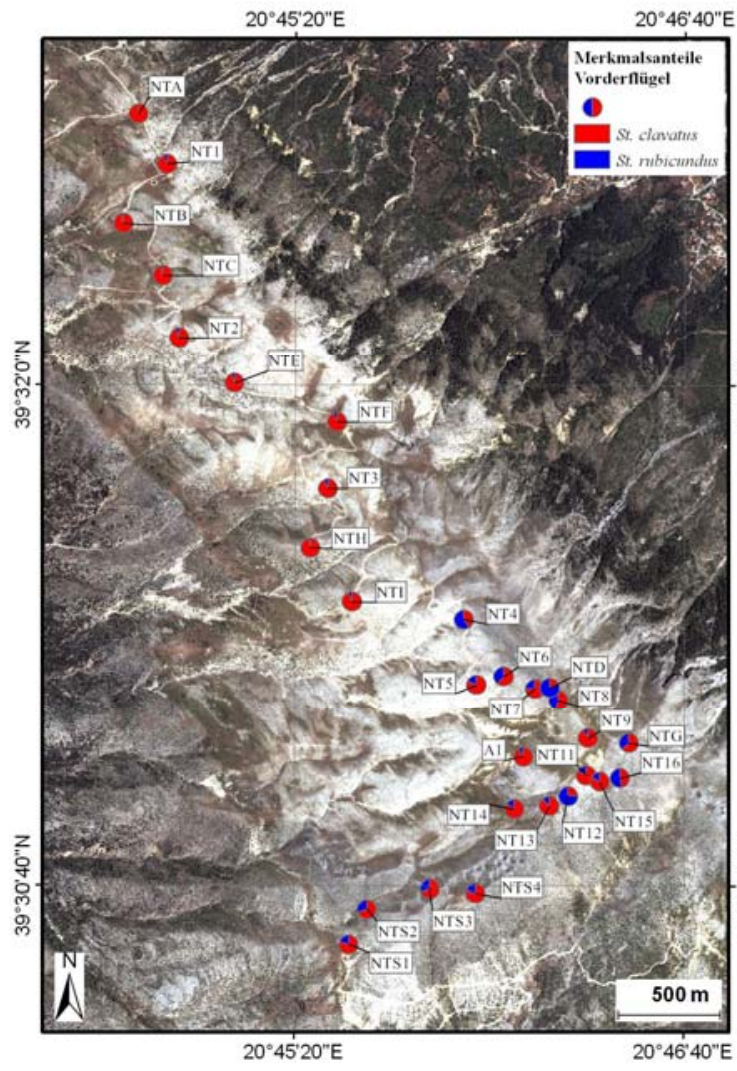

Abb. 46 Verteilung der durchschnittlichen Merkmalsanteile auf dem Nordtomaros.

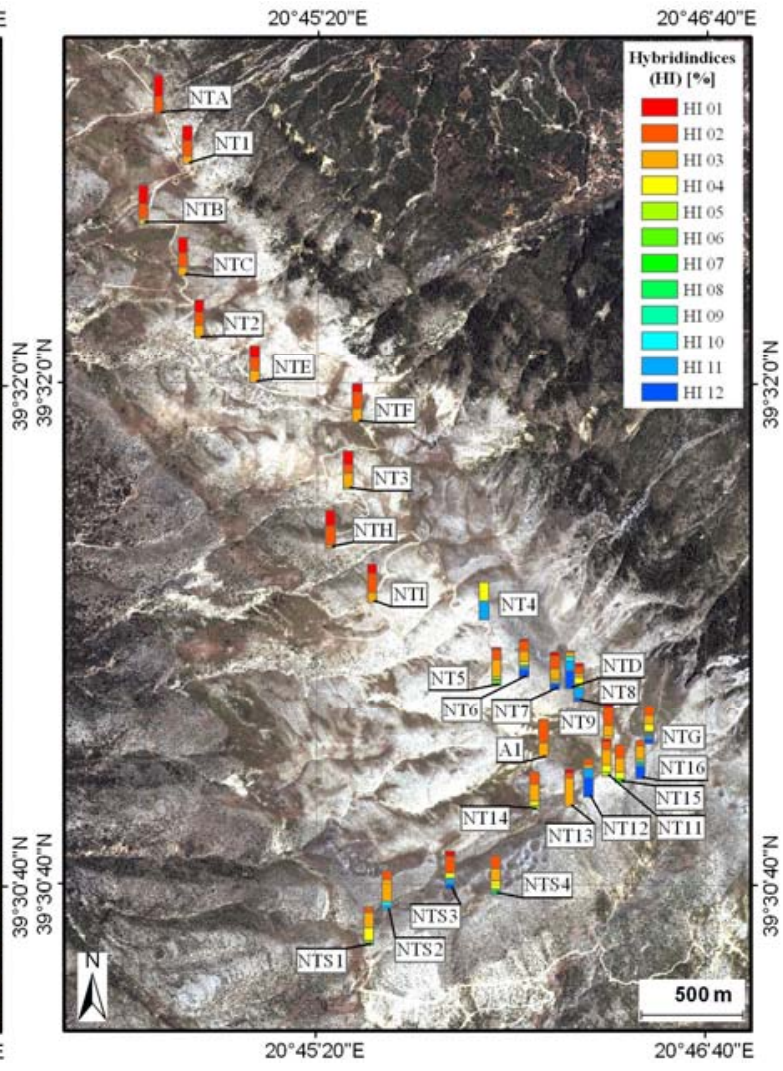

Abb. 47 Anteil der Individuen mit entsprechenden Hybridindices für jeden Fundort des Nordtomaros.

Merkmalsverteilung im Detail: Auf der nördlichen Hälfte des Nordtomaros, d. h. zwischen den GPS-Punkten NTA und NTI findet man, abgesehen von ganz wenigen Ausnahmen, ausschließlich Tiere, die entweder völlig der Xerovouni-Referenzpopulation von St. 
clavatus entsprechen (Index 1) oder ihr doch sehr nahestehen (Index 2 und 3). Dementsprechend beträgt in diesem Teilgebiet der durchschnittliche St. rubicundusMerkmalsanteil nur 5,12\%, wobei er am nördlichsten GPS-Punkt (NTA, $1270 \mathrm{~m}$ ) bei lediglich 1,7\% liegt. Nach Süden hin steigt er - leicht diskontinuierlich - auf über 7\% an (NT3 7,5\%; NTI 6,3\%) an. Die genaueren Werte sind der Tab. 11 zu entnehmen.

Tab. 11 Merkmalsverteilung der einzelnen Fundorte im nördlichen Teilgebiet des Nordtomaros

\begin{tabular}{|c|c|c|c|c|c|c|c|c|c|c|c|c|c|c|c|c|c|c|}
\hline \multicolumn{4}{|c|}{ Geographische Parameter } & \multicolumn{13}{|c|}{ Hybridindices } & \multicolumn{2}{|c|}{ Merkmalsanteil } \\
\hline GPS & Längengrad & Breitengrad & Höhe & 1 & 2 & 3 & 4 & 5 & 6 & 7 & 8 & 9 & 10 & 11 & 12 & $\Sigma$ & $\overline{\bar{x}}$ & $\sigma$ \\
\hline NTA & $39^{\circ} 32^{\prime} 43,2^{\prime \prime} \mathrm{N}$ & $20^{\circ} 44^{\prime} 47,7^{\prime \prime} \mathrm{E}$ & $1267 \mathrm{~m}$ & 10 & 8 & - & - & - & - & - & - & - & - & - & - & 18 & $1,70 \%$ & 2,6 \\
\hline NT1 & $39^{\circ} 32^{\prime} 35,2^{\prime \prime} \mathrm{N}$ & $20^{\circ} 44^{\prime} 53,6^{\prime \prime} \mathrm{E}$ & $1281 \mathrm{~m}$ & 16 & 17 & 8 & - & - & - & - & - & - & - & - & - & 41 & $4,70 \%$ & 5,5 \\
\hline NTB & $39^{\circ} 32^{\prime} 25,8^{\prime \prime} \mathrm{N}$ & $20^{\circ} 44^{\prime} 44,6^{\prime \prime} \mathrm{E}$ & $1330 \mathrm{~m}$ & 7 & 5 & 1 & 1 & - & - & - & - & - & - & - & - & 14 & $4,00 \%$ & 7,8 \\
\hline NTC & $39^{\circ} 32^{\prime} 17,5^{\prime \prime} \mathrm{N}$ & $20^{\circ} 44^{\prime} 52,8^{\prime \prime} \mathrm{E}$ & $1381 \mathrm{~m}$ & 8 & 7 & 4 & - & - & - & - & - & - & - & - & - & 19 & $4,30 \%$ & 5,2 \\
\hline NT2 & $39^{\circ} 32^{\prime} 07,6^{\prime \prime} \mathrm{N}$ & $20^{\circ} 44^{\prime} 56,1^{\prime \prime} \mathrm{E}$ & $1419 \mathrm{~m}$ & 4 & 4 & 4 & - & - & - & - & - & - & - & - & - & 12 & $7,00 \%$ & 7,6 \\
\hline NTE & $39^{\circ} 32^{\prime} 00,3^{\prime \prime} \mathrm{N}$ & $20^{\circ} 45^{\prime} 07,5^{\prime \prime} \mathrm{E}$ & $1453 \mathrm{~m}$ & 5 & 5 & 5 & - & - & - & - & - & - & - & - & - & 15 & $90 \%$ & 6,5 \\
\hline NTF & $39^{\circ} 31^{\prime} 54,1^{\prime \prime} \mathrm{N}$ & $20^{\circ} 45^{\prime} 28,7^{\prime \prime} \mathrm{E}$ & $1456 \mathrm{~m}$ & 3 & 6 & 5 & - & - & - & - & - & - & - & - & - & 14 & $6,70 \%$ & 5,4 \\
\hline NT3 & $39^{\circ} 31^{\prime} 43,4^{\prime \prime} \mathrm{N}$ & $20^{\circ} 45^{\prime} 26,9^{\prime \prime} \mathrm{E}$ & $1516 \mathrm{~m}$ & 8 & 5 & 8 & 1 & - & - & - & - & - & - & - & - & 22 & $7,50 \%$ & 7,8 \\
\hline NTH & $39^{\circ} 31^{\prime} 34,0^{\prime \prime} \mathrm{N}$ & $20^{\circ} 45^{\prime} 23,2^{\prime \prime} \mathrm{E}$ & $1505 \mathrm{~m}$ & 5 & 6 & 1 & - & - & - & - & - & - & - & - & - & 12 & $3,10 \%$ & 4,2 \\
\hline NTI & $39^{\circ} 31^{\prime} 25,4^{\prime \prime} \mathrm{N}$ & $20^{\circ} 45^{\prime} 31,8^{\prime \prime} \mathrm{E}$ & $1554 \mathrm{~m}$ & 5 & 10 & 5 & - & - & - & - & - & - & - & - & - & 20 & $6,30 \%$ & 5,2 \\
\hline
\end{tabular}

Die Teilpopulationen an den 19 GPS-Punkten im südlichen Teil des Nordtomaros, beginnend mit NT4 und endend mit NTS1, sind wesentlich heterogener zusammengesetzt als die des Nordteils. Tiere, die reinen St. clavatus entsprechen (Index 1), kommen nur selten vor bzw. fehlen an mehreren Standorten völlig. Hingegen sind Tiere mit Hybridindex 2, 3 und 4, in einigen Fällen auch vereinzelt mit Index 5, häufig vertreten. Vor allem kommen nun, wenn auch in geringer Zahl, auch Tiere hinzu, die als St. rubicundus (Index 12) oder dieser Art nahestehend (Indices 9, 10, 11) einzuordnen sind. Der auf St. rubicundus bezogene Merkmalsanteil liegt im südlichen Nordtomaros bei durchschnittlich 32,78\%, wobei er zwischen den einzelnen GPS-Punkten von 8,5\% (NT9) bis 83,1\% (NTD) variiert. Es gibt in diesem Gebiet nur wenige Fundorte an denen Individuen mit St. clavatus-, St. rubicundus- und intermediären Merkmalen zu finden sind. Man findet diese Verteilung im Bereich des GPS-Punktes NTG, hier liegt der Merkmalsanteil 37,5 \%. Ein Bezug zwischen Höhenlage und Merkmalsanteil kann im Bereich den Nord-Tomaros nicht gefunden werden. Im Einzelnen sind die Werte in der Tab. 12 dokumentiert. 
Tab. 12 Merkmalsverteilung der einzelnen Fundorte im südlichen Teilgebiet des Nordtomaros.

\begin{tabular}{|c|c|c|c|c|c|c|c|c|c|c|c|c|c|c|c|c|c|c|}
\hline \multicolumn{4}{|c|}{ Geographische Parameter } & \multicolumn{13}{|c|}{ Hybridindices } & \multicolumn{2}{|c|}{ Merkmalsanteil } \\
\hline GPS & Längengrad & Breitengrad & Höhe & 1 & 2 & 3 & 4 & 5 & 6 & 7 & 8 & 9 & 10 & 11 & 12 & $\Sigma$ & $\overline{\mathrm{x}}$ & $\sigma$ \\
\hline NT4 & & "E & $1621 \mathrm{~m}$ & - & - & - & 1 & - & - & - & - & - & - & 1 & - & 2 &, $10 \%$ & $\underline{48}$ \\
\hline NT6 & & $\mathrm{E}$ & $20 \mathrm{~m}$ & 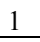 & E & 5 & 2 & 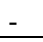 & - & - & 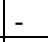 & 1 & - & 1 & 4 & 19 & & 172 \\
\hline NT5 & & $\mathrm{E}$ & $67 \mathrm{~m}$ & & 7 & 13 & 2 & 1 & 3 & 1 & - & 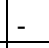 & - & - & - & 29 & & 15 \\
\hline NTD & $39^{\circ} 3$ & $20^{\circ} 46^{\prime}$ & $1757 \mathrm{~m}$ & & 1 & 2 & - & - & - & - & - & 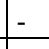 & 2 & 5 & 9 & 19 & $3,10 \%$ & 33 \\
\hline NT7 & $39^{\circ} 3$ & $20^{\circ} 46^{\prime}$ & $1769 \mathrm{~m}$ & 2 & 0 & 5 & 1 & 1 & - & - & - & - & - & - & 3 & 18 & $25,20 \%$ & 36 \\
\hline NT8 & & $\mathrm{E}$ & $1747 \mathrm{~m}$ & & 2 & 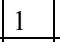 & 2 & 1 & - & - & - & - & - & 3 & 2 & 11 & & 42 \\
\hline NT9 & $39^{\circ} 3$ & $20^{\circ} 46$ & $1775 \mathrm{~m}$ & 3 & 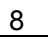 & 5 & 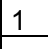 & - & - & - & - & - & - & - & - & 17 & 8 & \\
\hline NTG & $39^{\circ} 3$ & $20^{\circ} 46$ & $1765 \mathrm{~m}$ & & 13 & 13 & 10 & 1 & - & 1 & 2 & 1 & 2 & 3 & 7 & 53 & $37,00 \%$ & 37 \\
\hline A1 & & & $1704 \mathrm{~m}$ & - & 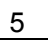 & 3 & - & - & - & - & - & - & - & - & 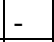 & 8 & & 3,6 \\
\hline NT11 & $\mathrm{N}$ & $20^{\circ} 46$ & $1776 \mathrm{~m}$ & 1 & 2 & 3 & 3 & 1 & - & - & - & - & - & - & - & 10 & & 9,6 \\
\hline NT16 & $2 " \mathrm{~N}$ & $20^{\circ} 46^{\prime} 27,0^{\prime \prime} \mathrm{E}$ & $1759 \mathrm{~m}$ & $\begin{array}{lll}- & \\
\end{array}$ & 2 & 5 & - & 1 & - & - & 1 & - & - & 1 & 5 & 15 & $52,30 \%$ & 42 \\
\hline NT15 & $39^{\circ} 30$ & $20^{\circ} 46$ & $1759 \mathrm{~m}$ & 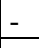 & 4 & 4 & 2 & 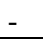 & - & 1 & - & - & - & - & - & 11 & $16,90 \%$ & 16 \\
\hline NT12 & $3 " \mathrm{~N}$ & $20^{\circ} 46$ & $1761 \mathrm{~m}$ & - & 2 & 1 & - & - & - & - & - & - & - & 3 & 6 & 12 & & 40 \\
\hline NT13 & 7"N & $20^{\circ} 46$ & $1738 \mathrm{~m}$ & 1 & 1 & 6 & - & - & - & - & - & - & - & - & - & 8 & $0 \%$ & 6,5 \\
\hline NT14 & $39^{\circ} 30$ & $20^{\circ} 46$ & $1684 \mathrm{~m}$ & $t$ & 3 & 4 & 1 & 1 & - & - & - & - & - & - & - & 9 & $14,70 \%$ & 9,9 \\
\hline NTS3 & $6 " \mathrm{~N}$ & $20^{\circ} 45^{\prime} 4$ & $1650 \mathrm{~m}$ & 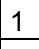 & 3 & - & 1 & - & - & - & - & - & - & 1 & 1 & 7 & $31,20 \%$ & 46 \\
\hline NTS4 & $39^{\circ} 30^{\prime} 38,9^{\prime \prime} \mathrm{N}$ & $20^{\circ} 45^{\prime} 57,3^{\prime \prime} \mathrm{E}$ & $1689 \mathrm{~m}$ & 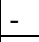 & 6 & 6 & 4 & - & - & - & 1 & 1 & - & - & - & 18 & $20,30 \%$ & 20 \\
\hline NTS2 & $39^{\circ} 30^{\prime} 36,4^{\prime \prime} \mathrm{N}$ & $20^{\circ} 45^{\prime} 35,0^{\prime \prime} \mathrm{E}$ & $1603 \mathrm{~m}$ & - & 2 & 5 & - & - & - & - & - & - & 1 & 1 & - & 9 & $29,30 \%$ & 34 \\
\hline NTS1 & $39^{\circ} 30^{\prime} 30,7^{\prime \prime} \mathrm{N}$ & $20^{\circ} 45^{\prime} 31,3^{\prime \prime} \mathrm{E}$ & $1540 \mathrm{~m}$ & $5^{-}$ & 2 & 6 & 5 & 1 & - & 1 & - & - & - & - & - & 15 & $20,60 \%$ & 13 \\
\hline
\end{tabular}

\subsubsection{Merkmalsverteilung auf dem Nord- und Südhang des Tomaros-Südmassiv}

Allgemeine Übersicht: In diesem Teil des Südmassivs wurden 103 GPS-Punkte gesetzt und insgesamt 1519 Individuen flügelmorphometrisch untersucht. Wie Abb. 48 zeigt, sind sämtliche zwölf Hybridindices vertreten. Es ergibt sich Folgendes: Anders als auf dem Nordtomaros ist hier mit 0,8 \% (12 Individuen) der Anteil der Individuen, die Hybridindex 1 zuzuordnen sind, also der St. clavatus -Referenzgruppe entsprechen, sehr klein. Einen Hybridindex von 2 haben 6,2 \% (94 Individuen) der untersuchten Individuen dieses Gebietes. Stark vertreten sind Tiere mit Hybridindex 3 (16\%, d. h. 235 Individuen) sowie Index 4 (15,6 \%, d. h. 243 Individuen) und solche mit Index 5 (10,5\%, d. h. 160 Individuen) Tiere mit Hybridindices 6 - 9 finden sich in deutlich geringerem Umfang: Index 6: 7,2\% (109 Individuen); Index 7: 3,9\% (59 Individuen), Index 8: 2,8\% (43 Individuen); Index 9: 4,7\% (71 Individuen). In größerem Umfang finden sich dann jedoch Tiere, die der St. rubicundus-Referenzgruppe ähneln (Index 10: 8,2\%, d. h. 124 Individuen; Index 11: 13\%, d. h. 197 Individuen), und immerhin 11,3\% (172 Individuen) entsprachen der St. rubicundusReferenzgruppe. 


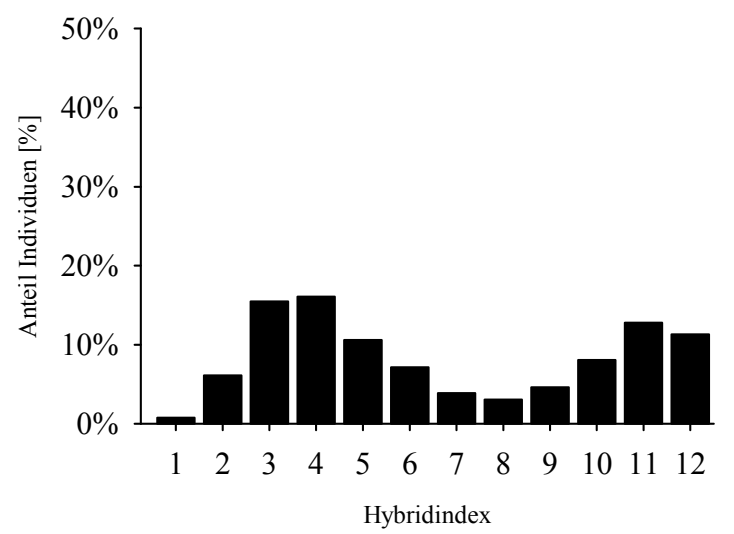

Abb. 48 Gesamtverteilung der Hybridindices (Vorderflügelmerkmal) auf dem Süd-Tomaros

Zusammenfassend ist festzustellen, dass es auf dem Südmassiv zwar relativ viele Individuen gibt, die den St. clavatus der Xerovouni-Referenzpopulation mehr oder weniger ähneln (Hybridindices $2-6$ ), jedoch anders als auf dem Nordtomaros, nur verschwindend wenige, nämlich $0,8 \%$, die jenen voll entsprechen (Index 1). Im Gegensatz dazu gibt es nicht nur eine größere Anzahl von Individuen, die den St. rubicundus der Referenzpopulation weitgehend gleichen (Hybridindices 9 - 11), sondern auch immerhin 11,3\%, die jenen voll zuzurechnen sind (Hybridindex 12).

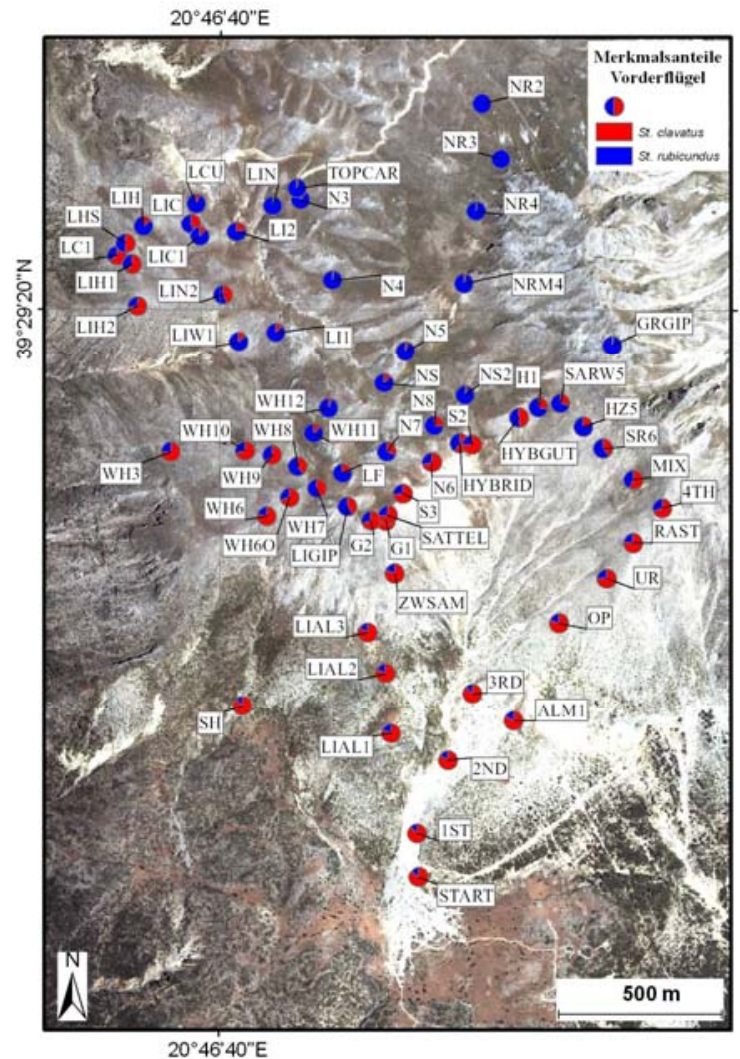

Abb. 49 Verteilung der durchschnittlichen

Merkmalsanteile auf dem Südtomaros.

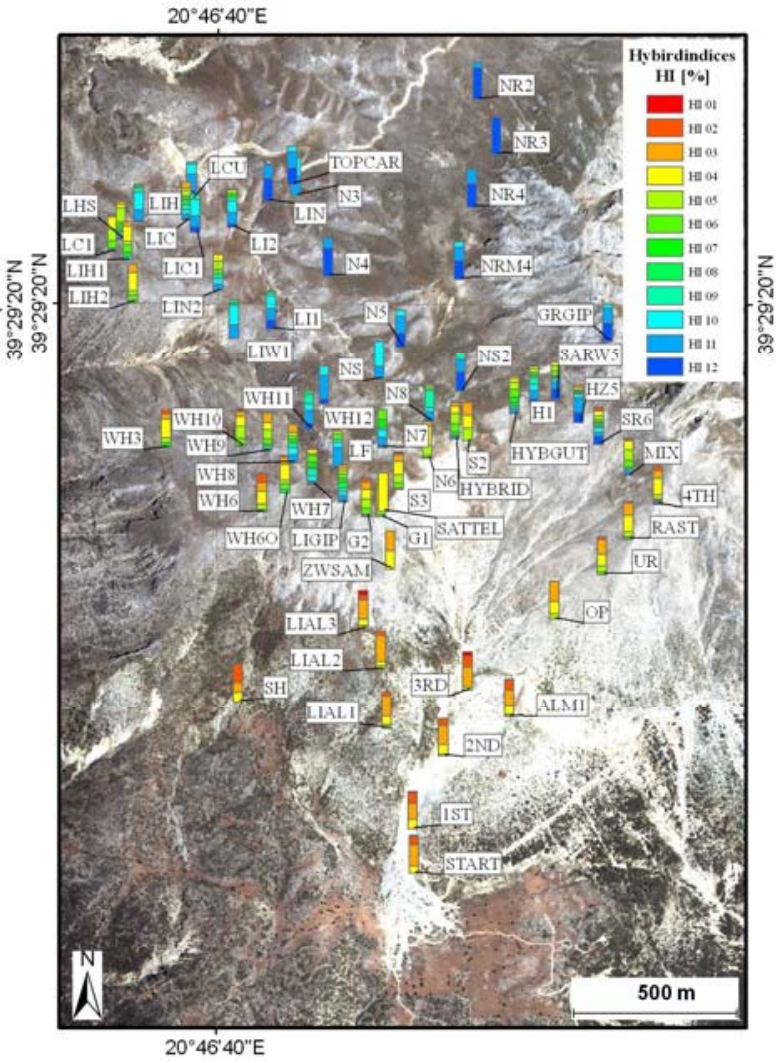

Abb. 50 Anteil der Individuen mit entsprechenden Hybridindices für jeden Fundort des Südtomaros. 
Merkmalsverteilung im Detail: Wie die Abb. 49 und Abb. 50 illustrieren, lässt sich das zweite Untersuchungsgebiet hinsichtlich der Merkmalsverteilung in drei Teilgebiete untergliedern: den Nord- und den Südhang sowie die dazwischen liegende Grenzzone. Im nördlichen Teil des Südtomaros, ausschließlich Individuen zu finden, die auf Basis der Flügelmorphometrie der St. rubicundus-Referenzgruppe entsprechen (Hybridindex 12) oder dieser sehr ähneln (Index 11). Sehr vereinzelt trifft man auch auf Individuen mit einem Hybridindex von 9 oder 10. Der durchschnittliche Merkmalsanteil der Individuen an diesen Fundorten dieses Gebietes liegt bei 97,63\% also nahe dem der Referenzpopulation von St. rubicundus, wobei der Merkmalsanteil an den einzelnen GPS-Punkten zwischen 100\% (NR2) und 96,7\% (NR4) variiert (Tab. 13).

Tab. 13 Merkmalsverteilung der einzelnen Fundorte im nördlichen Teilgebiet des Südtomaros.

\begin{tabular}{|c|c|c|c|c|c|c|c|c|c|c|c|c|c|c|c|c|c|c|}
\hline \multicolumn{4}{|c|}{ Geographische Parameter } & \multicolumn{13}{|c|}{ Hybridindices } & \multicolumn{2}{|c|}{ Merkmalsanteil } \\
\hline GPS & Cängengrad & Breitengrad & Höhe & 1 & & 3 & 4 & 5 & 0 & & & 9 & 10 & 11 & 12 & $\Sigma$ & $\bar{x}$ & $\sigma$ \\
\hline NR2 & $\mathrm{N}$ & "E & $1636 \mathrm{~m}$ & - & & 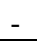 & . & 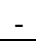 & 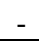 & & & - & - & 1 & 6 & 7 & $.0 \%$ & 1,5 \\
\hline NR3 & $0 " \mathrm{~N}$ & $20^{\circ} 47$ & $1717 \mathrm{~m}$ & - & & & - & - & - & & & 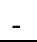 & - & - & 2 & 2 & $00,00 \%$ & 0 \\
\hline NR4 & ${ }^{\circ} 29 ' 29,8$ "N & $2,9 " \mathrm{E}$ & $1761 \mathrm{~m}$ & - & & _- & - & - & - & & & - & - & 3 & 5 & 8 & $8,50 \%$ & 2,6 \\
\hline NRM4 & 3902 & 3"E & $1811 \mathrm{~m}$ & - & & - & - & - & - & & & - & 2 & 5 & 7 & 14 & $6,70 \%$ & 4,8 \\
\hline & $9 " \mathrm{~N}$ & $20^{\circ} 46$ & $1673 \mathrm{~m}$ & - & & 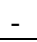 & - & - & - & & & - & - & 1 & 3 & 4 & $0 \%$ & 3,2 \\
\hline TOPCAR & $39^{\circ} 29^{\prime} 32,1^{\prime \prime N}$ & $20^{\circ} 46^{\prime} 49,8^{\prime \prime} \mathrm{E}$ & $1564 \mathrm{~m}$ & - & & - & - & - & - & & & - & 1 & 3 & 3 & 5 & $00 \%$ & 5,6 \\
\hline LIN & $39^{\circ} 29^{\prime} 30,3^{\prime \prime} \mathrm{N}$ & $20^{\circ} 46^{\prime} 46,7^{\prime \prime} \mathrm{E}$ & $1555 \mathrm{~m}$ & - & - & - & - & - & - & - & - & 1 & - & 12 & 21 & 34 & $97,20 \%$ & 5 \\
\hline
\end{tabular}

Die Teilpopulation (Tab. 14) die auf dem Südhang des Südmassives zu finden ist, zeichnet sich durch Individuen aus, die der Art St. clavatus nahestehen und hauptsächlich den Hybridindices von 2-5, vereinzelt auch Index 6, zuzuordnen sind. Tiere die der St. clavatusReferenzgruppe ganz entsprechen, sind in diesem Gebiet nur sehr selten zu finden (8 Individuen).

Die Merkmalsanteile der Tiere an diesen Fundorten liegen bei durchschnittlich 16,28\%, wobei sie in niedrigeren Höhenlagen zwischen 12,2 \% (SH) und 19,1\% (LIAL1) und in höheren zwischen 16,3\% (LIAL3) und 23,5\% (UR) variieren. 
Tab. 14 Merkmalsverteilung an den einzelnen Fundorten im südlichen Teilgebiet (Südhang) des Südtomaros.

\begin{tabular}{|c|c|c|c|c|c|c|c|c|c|c|c|c|c|c|c|c|c|c|}
\hline \multicolumn{4}{|c|}{ Geographische Parameter } & \multicolumn{13}{|c|}{ Hybridindices } & \multicolumn{2}{|c|}{ Merkmalsanteil } \\
\hline GPS & Längengrad & Breitengrad & Höhe & 1 & 2 & 3 & 4 & 5 & 6 & 7 & 8 & 9 & 10 & 11 & 12 & $\Sigma$ & $\overline{\mathrm{x}}$ & $\sigma$ \\
\hline UR & $39^{\circ} 28^{\prime} 53,2^{\prime \prime} \mathrm{N}$ & $20^{\circ} 47^{\prime} 29,7^{\prime \prime} \mathrm{E}$ & $1753 \mathrm{~m}$ & - & 1 & 5 & 4 & 2 & 1 & - & - & . & - & & - & 13 & $23,50 \%$ & 11 \\
\hline OP & $39^{\circ} 28^{\prime} 48,7^{\prime \prime} \mathrm{N}$ & $20^{\circ} 47^{\prime} 23,6^{\prime \prime} \mathrm{E}$ & $1644 \mathrm{~m}$ & - & - & 7 & 4 & 2 & - & - & - & - & - & - & - & 13 & $20,20 \%$ & 7,7 \\
\hline LIAL3 & $39^{\circ} 28^{\prime} 47,8^{\prime \prime} \mathrm{N}$ & $20^{\circ} 46^{\prime} 59,0^{\prime \prime} \mathrm{E}$ & $1611 \mathrm{~m}$ & 3 & 3 & 12 & 4 & 2 & - & - & - & - & - & - & - & 24 & $16,30 \%$ & 9,9 \\
\hline LIAL2 & $39^{\circ} 28^{\prime} 43,7^{\prime \prime} \mathrm{N}$ & $20^{\circ} 47^{\prime} 01,3 " \mathrm{E}$ & $1543 \mathrm{~m}$ & - & 1 & 7 & 1 & 1 & - & - & - & - & - & - & - & 10 & $16,90 \%$ & 8,9 \\
\hline 3RD & $39^{\circ} 28^{\prime} 41,6^{\prime \prime} \mathrm{N}$ & $20^{\circ} 47^{\prime} 12,5^{\prime \prime} \mathrm{E}$ & $1484 \mathrm{~m}$ & 1 & 3 & 5 & - & 1 & - & - & - & - & - & - & - & 10 & $12,30 \%$ & 11 \\
\hline SH & $39^{\circ} 28^{\prime} 40,4^{\prime \prime} \mathrm{N}$ & $20^{\circ} 46^{\prime} 43,0^{\prime \prime} \mathrm{E}$ & $1471 \mathrm{~m}$ & - & 4 & 2 & 2 & - & - & - & - & - & - & - & - & 8 & $12,20 \%$ & 8 \\
\hline ALM1 & $39^{\circ} 28^{\prime} 38,9^{\prime \prime} \mathrm{N}$ & $20^{\circ} 47^{\prime} 17,8^{\prime \prime} \mathrm{E}$ & $1514 \mathrm{~m}$ & 1 & 23 & 35 & 21 & 4 & 1 & - & - & - & - & - & - & 85 & $16,20 \%$ & 9 \\
\hline LIAL1 & $39^{\circ} 28^{\prime} 37,7^{\prime \prime} \mathrm{N}$ & $20^{\circ} 47^{\prime} 02,11^{\prime \prime} \mathrm{E}$ & $1476 \mathrm{~m}$ & - & 2 & 10 & 4 & 1 & 1 & - & - & - & - & - & - & 18 & $19,10 \%$ & 11 \\
\hline 2ND & $39^{\circ} 28^{\prime} 35,0^{\prime \prime} \mathrm{N}$ & $20^{\circ} 47^{\prime} 09,4^{\prime \prime} \mathrm{E}$ & $1425 \mathrm{~m}$ & - & 7 & 18 & 9 & 2 & - & - & - & - & - & - & - & 36 & $16,10 \%$ & 7,6 \\
\hline $1 \mathrm{ST}$ & $39^{\circ} 28^{\prime} 27,7^{\prime \prime} \mathrm{N}$ & $20^{\circ} 47^{\prime} 05,5^{\prime \prime} \mathrm{E}$ & $1368 \mathrm{~m}$ & 2 & 12 & 18 & 11 & - & - & - & - & - & - & - & - & 43 & $14,20 \%$ & 7,3 \\
\hline START & $39^{\circ} 28^{\prime} 23,3^{\prime \prime N}$ & $20^{\circ} 47^{\prime} 05,6^{\prime \prime} \mathrm{E}$ & $1326 \mathrm{~m}$ & 1 & 7 & 17 & 6 & - & - & - & - & - & - & - & - & 31 & $14,60 \%$ & 7,7 \\
\hline
\end{tabular}

. Zwischen Nord- und Südhang des Südmassivs erstreckt sich von der nord-westlichen Kante des Südtomaros über den westlichen kleinen Gipfel und den Sattel bis unterhalb des östlich gelegenen Hauptgipfels ein schmales Gebiet, in dem die Tiere sehr heterogene Merkmalscharaktere aufweisen (Abb. 49; Abb. 50). Hier wurden insgesamt 44 GPSPunkte gesetzt; Tab. 15).

Am nördlichen Rand dieses Gebietes (Abb. 49; Abb. 50) bei sechs der zwölf GPS-Punkte (LI2-WH11) findet man Individuen, die flügelmorphometrisch den Tieren der St. rubicundus-Referenzpopulation sehr nahe stehen (Index 9,11) oder ihnen, allerdings nur in wenigen Fällen, ganz entsprechen (Index 12). Sehr vereinzelt findet man aber auch Individuen die morphologisch sehr intermediäre Merkmale aufweisen (Index 5-8). Der durchschnittliche Merkmalsanteil liegt bei 90,68\%. Die an den etwas südlicher liegenden zwölf GPSPunkten (LIC-4TH) vorkommenden Tiere sind teilweise denen der St. clavatus- bzw. St. rubicundus-Vergleichspopulation ähnlich bzw. intermediär. Der Merkmalsanteil variiert zwischen 29,6\% und 80,8\%; durchschnittlich liegt er bei 53,69\%. Am südlichen Rand des Teilgebietes sind an den GPS-Punkten (LIH2 bis RAST) Individuen mit Merkmalen gefunden worden, die teilweise Gemeinsamkeiten mit der St. clavatus Referenzgruppe aufweisen (Indices 2-4) bzw. in der Mitte zwischen den beiden Referenzgruppen stehen (Indices 5-7). Der Merkmalsanteil variiert zwischen 19,3\% (ZWSAM) und 30,1\% (WH6O); der Mittelwert liegt bei $26,72 \%$. Auffällig ist auch hier, dass nur sehr vereinzelt Tiere gefunden worden sind, die St. clavatus voll entsprechen (2 Individuen); häufiger sind Individuen die der St. rubicundus-Referenzgruppe zuzuordnen sind. 
Tab. 15 Merkmalsverteilung an den einzelnen Fundorten zwischen Nord- und Südhang des Südtomaros.

\begin{tabular}{|c|c|c|c|c|c|c|c|c|c|c|c|c|c|c|c|c|c|c|}
\hline \multicolumn{4}{|c|}{ Geographische Parameter } & \multicolumn{13}{|c|}{ Hybridindices } & \multicolumn{2}{|c|}{ Merkmalsanteil } \\
\hline GPS & Längengrad & Breitengrad & Höhe & 1 & 2 & 3 & 4 & 5 & 6 & 7 & 8 & 9 & 10 & 11 & 12 & $\Sigma$ & $\overline{\mathrm{x}}$ & $\sigma$ \\
\hline LI2 & $39^{\circ} 29^{\prime} 27,7^{\prime \prime} \mathrm{N}$ & $20^{\circ} 46^{\prime} 42,1^{\prime \prime} \mathrm{E}$ & $1569 \mathrm{~m}$ & - & - & - & - & 1 & - & 1 & - & 1 & 3 & 3 & 1 & 10 & $78,80 \%$ & 21 \\
\hline LI1 & $39^{\circ} 29^{\prime} 17,7^{\prime \prime} \mathrm{N}$ & $20^{\circ} 46^{\prime} 47,2^{\prime \prime} \mathrm{E}$ & $1661 \mathrm{~m}$ & - & - & - & - & - & - & 1 & - & 2 & 9 & 8 & 6 & 26 & $89,10 \%$ & 11 \\
\hline LCU & $39^{\circ} 29^{\prime} 30,5^{\prime \prime} \mathrm{N}$ & $20^{\circ} 46^{\prime} 36,9^{\prime \prime} \mathrm{E}$ & $1545 \mathrm{~m}$ & - & - & - & - & - & - & - & - & 3 & 14 & 22 & 12 & 51 & $92,70 \%$ & 6,8 \\
\hline LIW1 & $39^{\circ} 29^{\prime} 16,7^{\prime \prime} \mathrm{N}$ & $20^{\circ} 46^{\prime} 42,4^{\prime \prime} \mathrm{E}$ & $1621 \mathrm{~m}$ & - & - & - & - & - & - & - & 1 & 1 & 7 & 5 & & 14 & $87,60 \%$ & 8,4 \\
\hline LIC1 & $39^{\circ} 29^{\prime} 27,3^{\prime \prime} \mathrm{N}$ & $20^{\circ} 46^{\prime} 37,3^{\prime \prime} \mathrm{E}$ & $1581 \mathrm{~m}$ & - & - & - & - & - & - & - & - & 2 & 6 & 4 & 2 & 14 & $88,80 \%$ & 7,8 \\
\hline LIH & $39^{\circ} 29^{\prime} 28,3^{\prime \prime} \mathrm{N}$ & $20^{\circ} 46^{\prime} 30,1^{\prime \prime} \mathrm{E}$ & $1543 \mathrm{~m}$ & - & - & - & - & - & - & 1 & 1 & 2 & 9 & 5 & 1 & 19 & $86,10 \%$ & 11 \\
\hline GRGIP & $39^{\circ} 29^{\prime} 16,4^{\prime \prime} \mathrm{N}$ & $20^{\circ} 47^{\prime} 30,3^{\prime \prime} \mathrm{E}$ & $1918 \mathrm{~m}$ & - & - & - & - & - & - & - & - & - & 1 & 4 & 5 & 10 & $97,70 \%$ & 4,2 \\
\hline N5 & $39^{\circ} 29^{\prime} 15,8^{\prime \prime} \mathrm{N}$ & $20^{\circ} 47^{\prime} 03,8^{\prime \prime} \mathrm{E}$ & $1757 \mathrm{~m}$ & - & - & - & - & - & - & - & - & - & 3 & 8 & 7 & 18 & $95,30 \%$ & 4,9 \\
\hline NS & $39^{\circ} 29^{\prime} 12,6^{\prime \prime} \mathrm{N}$ & $20^{\circ} 47^{\prime} 01,11^{\prime \prime} \mathrm{E}$ & $1745 \mathrm{~m}$ & - & - & - & - & - & - & - & - & - & 2 & 1 & - & 3 & $9,10 \%$ & 9,2 \\
\hline NS2 & $39^{\circ} 29^{\prime} 11,4^{\prime \prime} \mathrm{N}$ & $20^{\circ} 47^{\prime} 11,5^{\prime \prime} \mathrm{E}$ & $1778 \mathrm{~m}$ & - & - & - & - & - & - & - & - & 1 & - & 2 & 3 & 6 & $94,70 \%$ & 12 \\
\hline WH12 & $39^{\circ} 29^{\prime} 10,1^{\prime \prime} \mathrm{N}$ & $20^{\circ} 46^{\prime}$ & $1751 \mathrm{~m}$ & - & - & - & - & - & - & - & - & - & 4 & 10 & 2 & 16 & $94,50 \%$ & 5,4 \\
\hline WH11 & $39^{\circ} 29^{\prime} 07,6^{\prime \prime} \mathrm{N}$ & $20^{\circ} 46^{\prime} 52,0^{\prime \prime} \mathrm{E}$ & $1741 \mathrm{~m}$ & - & - & - & - & - & - & - & 1 & 3 & 2 & 4 & 2 & 12 & $88,00 \%$ & 11 \\
\hline LIC & $39^{\circ} 29^{\prime} 28,5^{\prime \prime} \mathrm{N}$ & $20^{\circ} 46^{\prime} 36,2^{\prime \prime} \mathrm{E}$ & $1568 \mathrm{~m}$ & - & - & 3 & 1 & 3 & 1 & 1 & 2 & 3 & 2 & 2 & 1 & 19 & $57,30 \%$ & 29 \\
\hline LHS & $39^{\circ} 29^{\prime} 26,6^{\prime \prime} \mathrm{N}$ & $20^{\circ} 46^{\prime} 27,8^{\prime \prime} \mathrm{E}$ & $1536 \mathrm{~m}$ & - & - & 2 & 3 & 10 & 2 & 3 & 2 & 1 & 2 & 2 & 1 & 28 & $0 \%$ & 26 \\
\hline LIN2 & $39^{\circ} 29^{\prime} 21,5^{\prime \prime} \mathrm{N}$ & $20^{\circ} 46^{\prime} 40,3^{\prime \prime} \mathrm{E}$ & $1631 \mathrm{~m}$ & - & - & 1 & 3 & 4 & 2 & - & - & 2 & 3 & 3 & - & 18 & $56,40 \%$ & 28 \\
\hline SARW5 & $39^{\circ} 29^{\prime} 10,6^{\prime \prime} \mathrm{N}$ & $20^{\circ} 47^{\prime} 23,7^{\prime \prime} \mathrm{E}$ & $1884 \mathrm{~m}$ & - & - & 1 & 4 & 6 & 6 & 1 & - & 2 & 3 & 9 & 12 & 44 & $71,00 \%$ & 31 \\
\hline H1 & $39^{\circ} 29^{\prime} 1$ & $20^{\circ} 47^{\prime} 20,9^{\prime \prime} \mathrm{E}$ & $1854 \mathrm{~m}$ & - & 1 & - & 3 & 4 & 3 & 1 & 3 & 8 & 7 & 10 & 5 & 5 & $70 \%$ & 26 \\
\hline N8 & $39^{\circ} 29^{\prime} 08,4^{\prime \prime} \mathrm{N}$ & $20^{\circ} 47^{\prime} 07,5^{\prime \prime} \mathrm{E}$ & $1780 \mathrm{~m}$ & - & - & 1 & - & 1 & - & - & 1 & 7 & 2 & 3 & 1 & 16 & $73,70 \%$ & 23 \\
\hline HZ5 & $39^{\circ} 29^{\prime} 08,3^{\prime \prime} \mathrm{N}$ & $20^{\circ} 47^{\prime} 26,6^{\prime \prime} \mathrm{E}$ & $1888 \mathrm{~m}$ & - & - & 2 & 2 & 1 & - & 1 & - & 1 & 3 & 11 & 8 & 29 & $80,80 \%$ & 29 \\
\hline HYBRID & $39^{\circ} 29$ & $20^{\circ} 47$ & $1790 \mathrm{~m}$ & - & 1 & 3 & 6 & 8 & 6 & 3 & - & - & 1 & 2 & - & 30 & $0 \%$ & 22 \\
\hline S2 & $39^{\circ} 29^{\prime} 06,5^{\prime \prime} \mathrm{N}$ & $20^{\circ} 47^{\prime} 12,4^{\prime \prime} \mathrm{E}$ & $1792 \mathrm{~m}$ & - & - & 1 & 1 & 1 & - & - & - & - & - & - & - & 3 & $24,50 \%$ & 9,1 \\
\hline SR6 & $39^{\circ} 29^{\prime} 06,1^{\prime \prime} \mathrm{N}$ & $20^{\circ} 47^{\prime} 29,2^{\prime \prime} \mathrm{E}$ & $1885 \mathrm{~m}$ & - & 4 & 2 & 6 & 5 & 3 & 2 & 3 & 2 & 6 & 5 & 11 & 49 & $61,60 \%$ & 34 \\
\hline WH10 & $39^{\circ} 29^{\prime} 05,8^{\prime \prime} \mathrm{N}$ & $20^{\circ} 46^{\prime} 43,2^{\prime \prime} \mathrm{E}$ & $1710 \mathrm{~m}$ & 1 & 1 & 3 & 6 & 8 & 4 & - & 1 & - & - & - & - & 24 & $9,70 \%$ & 14 \\
\hline N7 & $39^{\circ} 29^{\prime} 05,8^{\prime \prime} \mathrm{N}$ & $20^{\circ} 47^{\prime} 01,5^{\prime \prime} \mathrm{E}$ & $1787 \mathrm{~m}$ & - & - & - & - & - & 4 & 3 & - & 1 & 3 & 3 & 1 & 15 & $70,10 \%$ & 23 \\
\hline WH3 & $39^{\circ} 29^{\prime} 05,7^{\prime \prime} \mathrm{N}$ & $20^{\circ} 46^{\prime} 33,7^{\prime \prime} \mathrm{E}$ & $1617 \mathrm{~m}$ & - & 1 & 1 & 4 & 1 & - & 1 & - & - & - & - & - & 8 & $26,00 \%$ & 13 \\
\hline WH9 & $39^{\circ} 29^{\prime} 05,4^{\prime \prime} \mathrm{N}$ & $20^{\circ} 46^{\prime} 46,7^{\prime \prime} \mathrm{E}$ & $1736 \mathrm{~m}$ & - & - & 4 & 3 & 4 & 2 & 1 & - & 1 & 1 & - & - & 16 & $35,90 \%$ & 21 \\
\hline N6 & $39^{\circ} 29^{\prime} 04,7^{\prime \prime} \mathrm{N}$ & $20^{\circ} 47^{\prime} 07,3^{\prime \prime} \mathrm{E}$ & $1793 m$ & - & 1 & 8 & 28 & 16 & 3 & 1 & - & - & - & - & - & 57 & $28,20 \%$ & 9,4 \\
\hline WH8 & $39^{\circ} 29^{\prime} 04,3^{\prime \prime} \mathrm{N}$ & $20^{\circ} 46^{\prime} 50,0^{\prime \prime} \mathrm{E}$ & $1761 \mathrm{~m}$ & - & - & 2 & - & 1 & 1 & 1 & - & 1 & 2 & 2 & - & 10 & $60,70 \%$ & 31 \\
\hline LF & $39^{\circ} 29^{\prime} 03,6^{\prime \prime} \mathrm{N}$ & $20^{\circ} 46^{\prime} 55,7^{\prime \prime} \mathrm{E}$ & $1783 \mathrm{~m}$ & - & - & - & 1 & - & 2 & - & - & 1 & 2 & 7 & 1 & 14 & $80,60 \%$ & 25 \\
\hline WH7 & $39^{\circ} 29^{\prime} 02,1^{\prime \prime} \mathrm{N}$ & $20^{\circ} 46^{\prime} 52,5^{\prime \prime} \mathrm{E}$ & $1784 \mathrm{~m}$ & - & 2 & 1 & 5 & 2 & 7 & 6 & 2 & 8 & 5 & 2 & 2 & 42 & $57,50 \%$ & 25 \\
\hline LIGIP & $39^{\circ} 29^{\prime} 00,3^{\prime \prime} \mathrm{N}$ & $20^{\circ} 46^{\prime} 56,4^{\prime \prime} \mathrm{E}$ & $1803 \mathrm{~m}$ & 1 & 1 & 11 & 6 & 10 & 12 & 13 & 15 & 11 & 11 & 13 & 6 & 110 & $58,90 \%$ & 27 \\
\hline LC1 & $39^{\circ} 29^{\prime} 25,4^{\prime \prime} \mathrm{N}$ & $20^{\circ} 46^{\prime} 26,7^{\prime \prime} \mathrm{E}$ & $1536 \mathrm{~m}$ & - & 1 & 1 & 7 & 7 & 4 & 1 & - & - & - & 1 & - & 22 & $35,30 \%$ & 18 \\
\hline LIH1 & $39^{\circ} 29^{\prime} 24,5^{\prime \prime} \mathrm{N}$ & $20^{\circ} 46^{\prime} 28,7^{\prime \prime} \mathrm{E}$ & $1555 \mathrm{~m}$ & - & - & 1 & 4 & 1 & 2 & 1 & - & - & 1 & - & - & 10 & $37,70 \%$ & 21 \\
\hline MIX & $39^{\circ} 29^{\prime} 03,0^{\prime \prime} \mathrm{N}$ & $20^{\circ} 47^{\prime} 33,1^{\prime \prime} \mathrm{E}$ & $1880 \mathrm{~m}$ & - & 1 & 1 & 5 & 6 & 4 & 1 & - & 1 & - & 2 & 1 & 22 & $42,60 \%$ & 26 \\
\hline 4TH & $39^{\circ} 29^{\prime} 00,2^{\prime \prime} \mathrm{N}$ & $20^{\circ} 47^{\prime} 36,8^{\prime \prime} \mathrm{E}$ & $1876 \mathrm{~m}$ & - & 3 & 6 & 12 & 3 & 1 & - & 1 & - & - & 1 & 1 & 28 & $29,60 \%$ & 23 \\
\hline LIH2 & $39^{\circ} 29^{\prime} 20,3^{\prime \prime} \mathrm{N}$ & $20^{\circ} 46^{\prime} 29,4^{\prime \prime} \mathrm{E}$ & $1581 \mathrm{~m}$ & 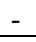 & - & 4 & 8 & 2 & 3 & - & - & - & - & - & - & 17 & $27,00 \%$ & 9,4 \\
\hline WH6O & $39^{\circ} 29^{\prime} 01,2^{\prime \prime} \mathrm{N}$ & $20^{\circ} 46^{\prime} 48,9^{\prime \prime} \mathrm{E}$ & $1768 \mathrm{~m}$ & - & 1 & 4 & 9 & 2 & 3 & - & 2 & - & - & - & - & 21 & $30,10 \%$ & 15 \\
\hline WH6 & $39^{\circ} 28^{\prime} 59,3^{\prime \prime} \mathrm{N}$ & $20^{\circ} 46^{\prime} 46,0^{\prime \prime} \mathrm{E}$ & $1750 \mathrm{~m}$ & - & 4 & 4 & 6 & 3 & 1 & - & - & - & - & - & - & 18 & $22,00 \%$ & 12 \\
\hline S3 & $39^{\circ} 29^{\prime} 01,6^{\prime \prime} \mathrm{N}$ & $20^{\circ} 47^{\prime} 03,5^{\prime \prime} \mathrm{E}$ & $1792 \mathrm{~m}$ & 1 & 1 & 4 & 8 & 5 & 3 & 1 & - & - & - & - & - & 23 & $27,20 \%$ & 14 \\
\hline SATTEL & $39^{\circ} 28^{\prime} 59,5^{\prime \prime} \mathrm{N}$ & $20^{\circ} 47^{\prime} 01,6^{\prime \prime} \mathrm{E}$ & $1786 \mathrm{~m}$ & - & - & - & 1 & - & - & - & - & - & - & - & - & 1 & $26,20 \%$ & - \\
\hline ZWSAM & $39^{\circ} 28^{\prime} 53,6^{\prime \prime} \mathrm{N}$ & $20^{\circ} 47^{\prime} 02,5^{\prime \prime} \mathrm{E}$ & $1715 \mathrm{~m}$ & - & - & 1 & 1 & - & - & - & - & - & - & - & - & 2 & $19,30 \%$ & 6,7 \\
\hline G2 & $39^{\circ} 28^{\prime} 58,9^{\prime \prime} \mathrm{N}$ & $20^{\circ} 46^{\prime} 59,4^{\prime \prime} \mathrm{E}$ & $1795 \mathrm{~m}$ & - & 3 & 7 & 9 & 8 & 6 & 1 & 1 & - & - & - & - & 35 & $29,20 \%$ & 14 \\
\hline G1 & $39^{\circ} 28^{\prime} 58,9^{\prime \prime} \mathrm{N}$ & $20^{\circ} 47^{\prime} 01,3^{\prime \prime} \mathrm{E}$ & $1785 \mathrm{~m}$ & - & - & 3 & 7 & 2 & 1 & - & - & - & - & - & - & 13 & $25,70 \%$ & 8,7 \\
\hline RAST & $39^{\circ} 28^{\prime} 56,7^{\prime \prime} \mathrm{N}$ & $20^{\circ} 47^{\prime} 33,1^{\prime \prime E}$ & $1812 \mathrm{~m}$ & - & 1 & 5 & 7 & 2 & - & 1 & - & - & - & - & - & 16 & $23,20 \%$ & 12 \\
\hline
\end{tabular}




\subsubsection{Merkmalsverteilung auf dem Osthang des Tomaros-Südmassiv}

Allgemeine Übersicht: Auf dem Osthang des Südmassivs wurden 39 GPS-Punkte (Tab. 12) gesetzt, an denen insgesamt 697 (M: 377; W:320) Individuen gesammelt und morphometrisch untersucht wurden, wobei sich eine zweigipfelige Verteilung ergab (Abb. 51). Mehr als zwei Drittel davon, nämlich 71,3\%, entsprechen den Tieren der St. rubicundusReferenzpopulation oder sind diesen sehr ähnlich (Index 12: 47,3\%; Index 11, 9,7\%, Index 10: 4,3\%). Tiere, die den Hybridindices 7 (0,7\%), 8 (0,7\%) und 9 (1,7\%) zugeordnet werden können, sind dagegen selten. Stärker vertreten, nämlich zu einem Viertel $(24,8 \%)$ sind dann erst wieder Individuen, welche den St. clavatus-Referenztieren ähneln (Index 2: 1,6\%, Index 3: 7,0\%, Index 4: 7,7\%) oder ausgesprochen intermediär (Index 5: 5,6\%, Index: 6: 2,9\%) sind. Im Gegensatz zu St. rubicundus kommen „reine“ St. clavatus (Index 1) praktisch gar nicht vor: Hiervon wurde nur ein einziges Tier gefunden.

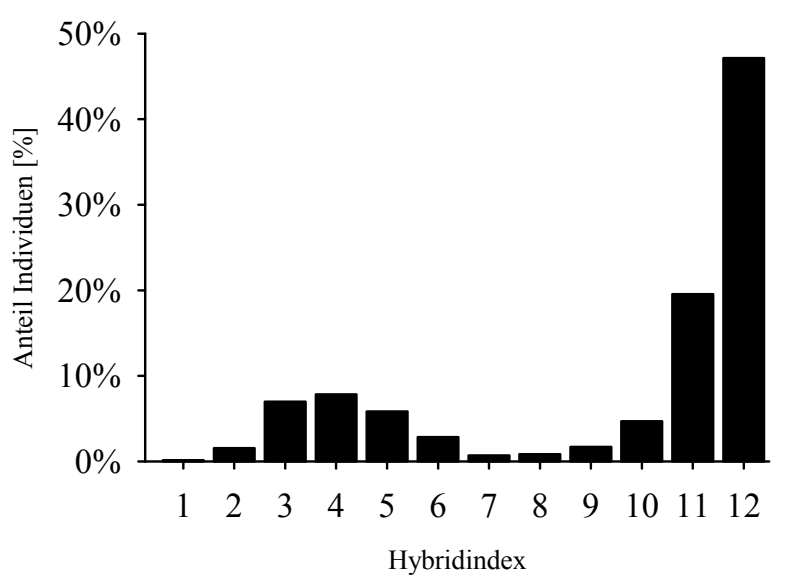

Abb. 51 Gesamtverteilung der Hybridindices (Vorderflügelmerkmal) auf dem Osthang des Süd-Tomaros. 


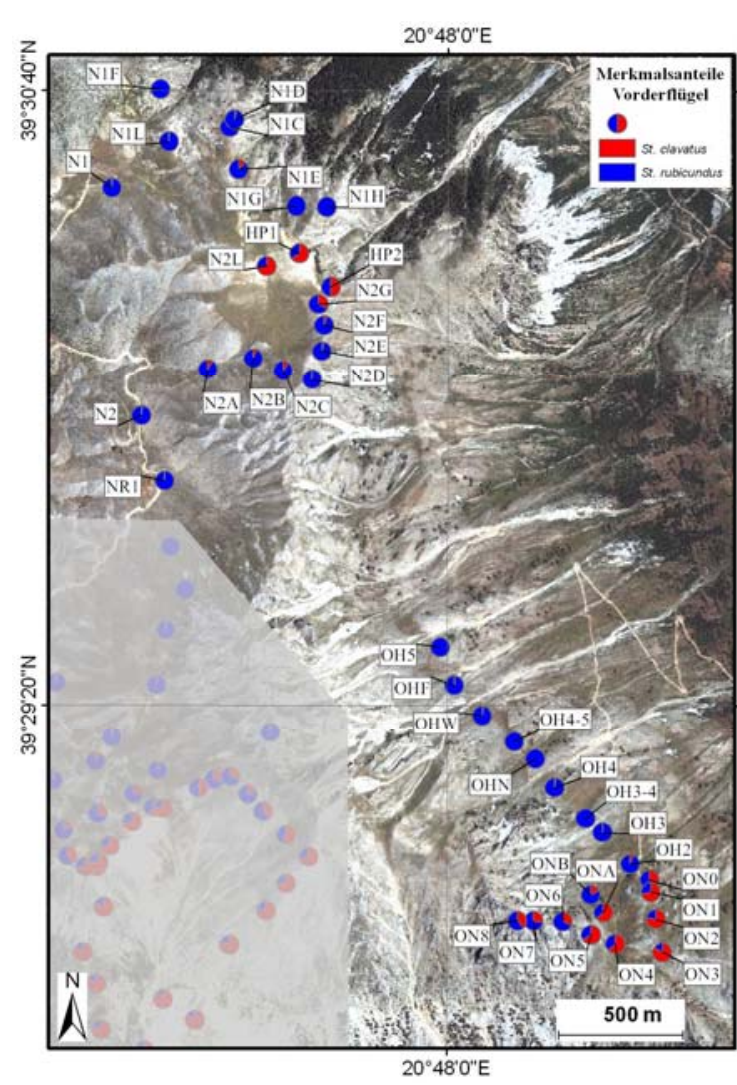

Abb. 52 Verteilung der durchschnittlichen Merkmalsanteile [\%] auf dem Osthang des Südtomaros.

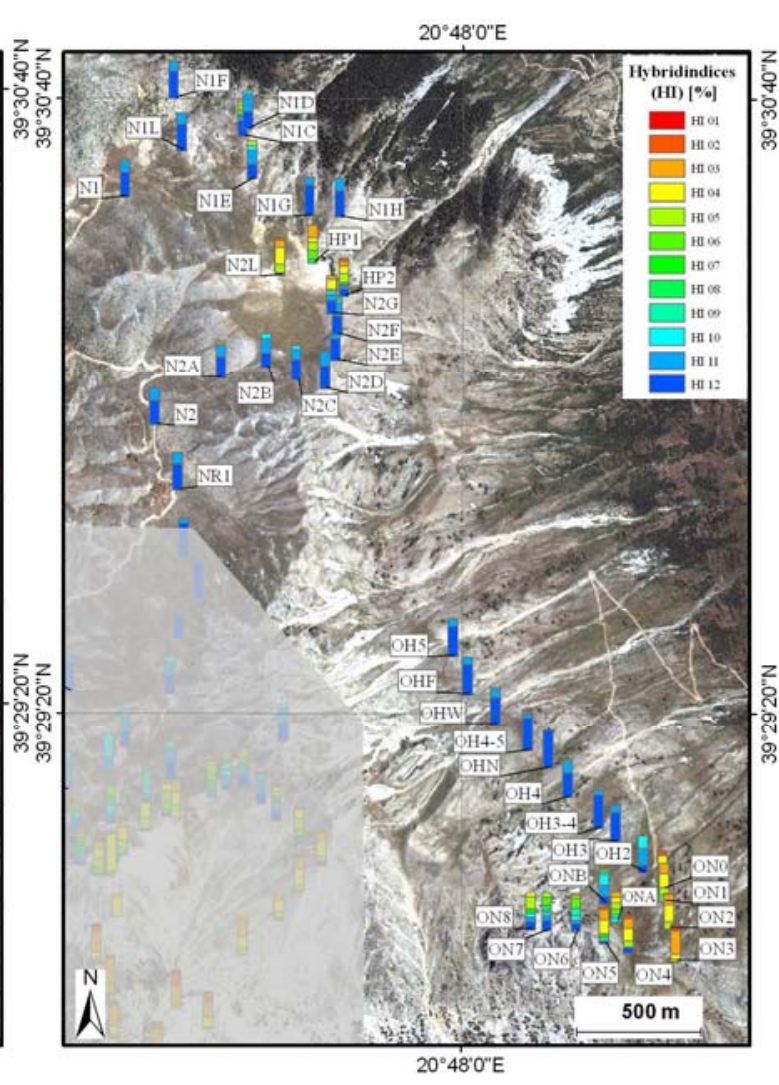

Abb. 53 Anteil der Individuen mit entsprechenden Hybridindices für jeden Fundort des Osthanges des Südtomaros.

Merkmalsverteilung im Detail: Der Osthang des Südmassivs kann in ein nördliches und ein südliches Teilgebiet unterteilt werden, in denen 20 bzw. 19 GPS-Punkte gesetzt wurden (Tab. 16 und Tab. 17). Die im nördlichen Teil zu findenden Individuen sind an 15 der 20 GPS-Punkte den Referenztieren der Art St. rubicundus sehr ähnlich (Hybridindex 10 und 11) oder entsprechen diesen vollständig (Index 12); Tiere mit niedrigeren Hybridindices, also stärkeren St. clavatus Merkmalen, kommen nur sehr vereinzelt vor. Somit beträgt der durchschnittliche Merkmalsanteil 96,83\%. Davon ausgenommen sind die Individuen, die an fünf GPS-Punkten gefunden wurden, die in der Mitte des weitgehend von St. rubicundus geprägten nördlichen Teilgebiets liegen. Die Merkmalsanteile betragen hier: N1C: 69,10\% HP1: 33,2\%; N2L: 27,0\%; HP2: 48,1\%, N2G: 68,5\%; im Mittel 52,17\%. 
Tab. 16 Merkmalsverteilung an den einzelnen Fundorten im nördlichen Teilgebiet des Osthanges

\begin{tabular}{|c|c|c|c|c|c|c|c|c|c|c|c|c|c|c|c|c|c|c|}
\hline \multicolumn{4}{|c|}{ eographische Parameter } & \multicolumn{13}{|c|}{ Hybridindices } & \multicolumn{2}{|c|}{ Merkmalsanteil } \\
\hline SPS & Längengrad & reitengrad & 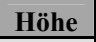 & 1 & 2 & 3 & 4 & 5 & 6 & 7 & 8 & 9 & 10 & 11 & 12 & $\Sigma$ & 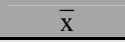 & $\sigma$ \\
\hline N1F & & & & & & - & & & & & & & & 3 & 8 & 11 & & - \\
\hline N1D & & & & & . & 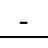 & - & 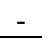 & 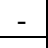 & & & 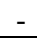 & 1 & 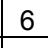 & 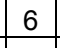 & 3 & & 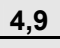 \\
\hline $\mathrm{V} 1 \mathrm{C}$ & & & & & - & 4 & 3 & & 5 & & & - & 1 & 5 & 18 & & & - \\
\hline N1L & J & E & $92 \mathrm{~m}$ & & - & - & & & - & & - & 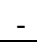 & - & 9 & 21 & 30 & & 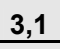 \\
\hline N1E & $\mathrm{N}$ & 21 & $45 \mathrm{~m}$ & & 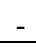 & - & - & 1 & - & & 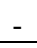 & 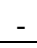 & 1 & 3 & 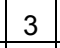 & 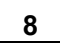 & & 1 \\
\hline N1 & & & & & 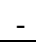 & - & - & & - & & 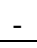 & - & 2 & 14 & 31 & & & \\
\hline N1G & & & & & 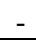 & - & - & & 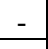 & & - & - & & & & & & \\
\hline N1H & N & $20^{\circ} 4$ & $68 \mathrm{~m}$ & - & - & - & & 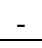 & - & & 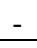 & - & - & 4 & 8 & 12 & & 8 \\
\hline HP1 & $\mathrm{N}$ & $20^{\circ} 47$ & & - & - & 3 & 1 & & 2 & & 1 & - & - & - & - & & & 7 \\
\hline N2L & & & & & 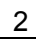 & 6 & - & & - & & & - & 1 & & - & & & \\
\hline HP2 & $\mathrm{N}$ & $20^{\circ} 4$ & $01 \mathrm{~m}$ & 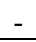 & 2 & 3 & & & 1 & & - & - & 1 & 2 & 5 & 25 & & 6 \\
\hline N2G & $\mathrm{N}$ & $20^{\circ}$ & & - & - & 2 & 5 & 1 & - & & - & - & 2 & 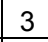 & 7 & 20 & & 7 \\
\hline $\mathrm{N} 2 \mathrm{~F}$ & & & & - & - & - & & & - & & & - & 1 & & & & & \\
\hline N2E & & $20^{\circ} 4$ & & 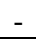 & - & - & & 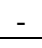 & - & & 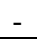 & - & 2 & 5 & 10 & 17 & & \\
\hline N2B & $\mathrm{N}$ & $20^{\circ}$ & $\mathrm{m}$ & 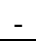 & - & - & - & - & - & & . & 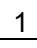 & 2 & 5 & 5 & 13 & &, 5 \\
\hline N2A & & & & 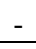 & 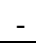 & - & 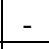 & 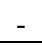 & - & & - & - & - & 4 & 7 & 11 & & \\
\hline N2C & $39^{\circ} 30$ & $20^{\circ} 47$ & $3 \mathrm{~m}$ & 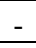 & - & - & 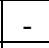 & - & - & & - & - & 3 & 7 & 14 & 26 & & 5,7 \\
\hline N2D & $39^{\circ} 30^{\prime} 02,4^{\prime \prime} \mathrm{N}$ & & & - & - & - & - & - & - & 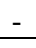 & 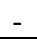 & - & - & 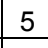 & 0 & 13 & & $=$ \\
\hline N2 & $39^{\circ} 29^{\prime} 5$ & $20^{\circ} 47^{\prime} 08,6^{\prime \prime} \mathrm{E}$ & $1524 \mathrm{~m}$ & - & - & - & - & - & - & - & - & - & 1 & 12 & 24 & 37 & $0 \%$ & 3,3 \\
\hline $\mathbf{R} 1$ & $1 " \mathrm{~N}$ & $20^{\circ} 47^{\prime} 12,5^{\prime \prime} \mathrm{E}$ & $578 \mathrm{~m}$ & - & 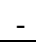 & 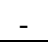 & - & 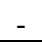 & 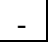 & - & - & - & - & 6 & 11 & 2 & $00 \%$ & 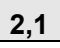 \\
\hline
\end{tabular}

Das südliche Teilgebiet des Osthangs lässt sich hinsichtlich der Merkmalsverteilung in zwei Bereiche unterteilen: In den nördlichen, wo 8 GPS-Punkte gesetzt wurden, sind überwiegend Tiere, die zum größten Teil ganz oder nahezu den Individuen der St. rubicundus-Referenzpopulation entsprechen, gefunden worden. Dementsprechend liegt der durchschnittliche Merkmalsanteil bei 98,66\%. Die Tiere im südlichen Teil, hier wurden elf GPSPunkte gesetzt, sind hinsichtlich ihrer Merkmalsanteile wesentlich heterogener. So findet man hier Tiere, die St. clavatus nahe stehen (Index 2; 3; 4) oder ausgesprochen intermediär sind (Index 5; 6; 7;8) bzw. hinsichtlich ihres Merkmalscharakters St. rubicundus nahestehen (Index 9 bis 11), in einigen Fällen sogar ihnen völlig entsprechen (Index 12). Hingegen fehlen Individuen, die der Referenzgruppe der Art St. clavatus entsprechen fast völlig. Die Merkmalsanteile der Tiere an den einzelnen Fundorten variieren in diesem Teilgebiet zwischen 16,5\%(ON3) und 83,2\% (ONB); im Durchschnitt liegt der Merkmalsanteil bei $45,05 \%$. 
Tab. 17 Merkmalsverteilung an den einzelnen Fundorten im südlichen Teilgebiet des Osthanges

\begin{tabular}{|c|c|c|c|c|c|c|c|c|c|c|c|c|c|c|c|c|c|c|}
\hline \multicolumn{4}{|c|}{ eographische Parameter } & \multicolumn{13}{|c|}{ Hybridindices } & \multicolumn{2}{|c|}{ Merkmalsanteil } \\
\hline GPS & Längengrad & Breitengrad & Höhe & 1 & 2 & 3 & 4 & 5 & 6 & 7 & 8 & 9 & 10 & 11 & 12 & $\Sigma$ & $\overline{\mathrm{x}}$ & $\sigma$ \\
\hline $\mathrm{OH} 5$ & "N & 21 & 14 & & - & - & & & - & & & 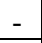 & - & 3 & 13 & 16 & $0 \%$ & 4 \\
\hline OHW & $\mathrm{N}$ & 2 & 14 & & - & - & & & - & & & 1 & - & 5 & 17 & 2 & &, 5 \\
\hline OH4-5 & & & & & & - & & & - & & & . & - & 3 & 21 & 24 & & \\
\hline OHN & $\mathrm{N}$ & 20 & $1360 \mathrm{~m}$ & - & - & - & - & & - & - & & - & - & - & 0 & 2 & & 0 \\
\hline $\mathrm{OH} 4$ & $39^{\circ} 29^{\prime} 09,2^{\prime \prime} \mathrm{N}$ & $20^{\circ} 48^{\prime}$ & $1358 \mathrm{~m}$ & & - & - & & & - & 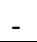 & & 1 & 1 & 8 & 17 & 27 & $\%$ & 5,5 \\
\hline $\mathrm{OH} 3-4$ & $39^{\circ} 29$ & & 13 & - & - & - & - & - & - & 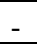 & & - & - & 3 & 20 & & & 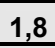 \\
\hline $\mathrm{OH} 3$ & $39^{\circ} 29$ & $20^{\circ}$ & $1321 \mathrm{~m}$ & & - & - & & & - & - & & - & 2 & 10 & 42 & - & & 2,7 \\
\hline $\mathrm{OH} 2$ & $39^{\circ} 28^{\prime}$ & $20^{\circ} 48$ & $1294 \mathrm{~m}$ & & - & - & & - & - & 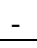 & 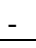 & - & 3 & 4 & 2 & 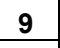 & $\%$ & 6,1 \\
\hline ONO & $39^{\circ} 28$ & $20^{\circ} 4$ & $1291 \mathrm{~m}$ & - & 1 & - & 2 & 1 & 1 & 1 & 1 & 1 & - & 1 & 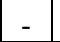 & 9 & & 8 \\
\hline ON1 & $39^{\circ} 28$ & $20^{\circ} 4$ & $1313 \mathrm{~m}$ & 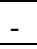 & - & 3 & 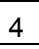 & 2 & 2 & 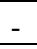 & & - & - & 4 & - & 11 & & 11 \\
\hline ONB & $39^{\circ} 28^{\prime}$ & $20^{\circ} 48$ & $1403 \mathrm{~m}$ & 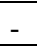 & 1 & - & & 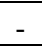 & - & - & 1 & 1 & 4 & 4 & 3 & 14 & $\%$ & 24 \\
\hline ONA & $39^{\circ} 28^{\prime}$ & $20^{\circ} 4$ & $1406 \mathrm{~m}$ & - & - & 7 & 3 & 3 & 3 & - & - & 2 & 2 & - & - & 20 & $\%$ & 25 \\
\hline ON2 & $39^{\circ} 28$ & $20^{\circ} 4$ & $1345 \mathrm{~m}$ & 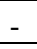 & 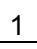 & 3 & 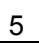 & & - & - & & - & - & - & - & & & 8,5 \\
\hline ON8 & $39^{\circ} 28^{\prime}$ & $20^{\circ} 48^{\prime}$ & $1576 \mathrm{~m}$ & - & - & - & 1 & 3 & - & 1 & - & - & 1 & 1 & 2 & 0 & $\%$ & 33 \\
\hline ON7 & $39^{\circ} 28^{\prime} 5$ & $20^{\circ} 48^{\prime}$ & $1547 \mathrm{~m}$ & 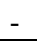 & - & - & & $\angle$ & 3 & - & 2 & 1 & 1 & 2 & 4 & 10 & $\%$ & 26 \\
\hline ON6 & $39^{\circ} 28^{\prime}$ & $20^{\circ} 48$ & $1497 \mathrm{~m}$ & - & 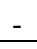 & 1 & 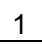 & - & 4 & - & - & 3 & 2 & 1 & 3 & 15 & $\%$ & 29 \\
\hline ON5 & $39^{\circ} 28^{\prime} 50,1^{\prime \prime} \mathrm{N}$ & $20^{\circ} 48^{\prime} 24,2^{\prime \prime} \mathrm{E}$ & $1455 \mathrm{~m}$ & 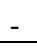 & 1 & 3 & 4 & 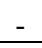 & - & 1 & - & 1 & - & - & 1 & 11 & $50 \%$ & 29 \\
\hline ON4 & $39^{\circ} 28^{\prime} 48,9^{\prime \prime} \mathrm{N}$ & $20^{\circ} 48^{\prime}$ & $1422 \mathrm{~m}$ & - & 1 & 3 & 4 & 2 & - & - & - & - & - & - & 2 & 12 & $\%$ & 31 \\
\hline ON3 & $39^{\circ} 28^{\prime} 47,9^{\prime \prime} \mathrm{N}$ & $20^{\circ} 48^{\prime} 36,2^{\prime \prime} \mathrm{E}$ & $1379 \mathrm{~m}$ & 1 & 2 & 10 & 2 & 1 & - & - & - & - & - & - & - & 16 & $16,50 \%$ & 9,4 \\
\hline
\end{tabular}

\subsubsection{Räumliches Verteilungsmuster und Kontaktzonen}

In diesem Kapitel soll die räumliche Verteilung des Vorderflügelmerkmals der Tiere auf dem Tomaros genauer untersucht und im Zuge dessen die Mittelpunkte der Hybridzone bestimmt werden. Für die Berechnung der Merkmalsverteilung wurden die Daten in einem geographischen Informationssystem (GIS) eingebunden und verrechnet.

Die Interpolation wird mit der GIS-Software ArcGIS durchgeführt, um einen Gesamteindruck über die Merkmalsverteilung der Vorderflügel der Tiere des gesamten Untersuchungsgebiets des Tomaros zu erhalten. Dies ist nötig, da es sehr schwierig ist, das gesamte, teils schwer zugängliche, Gebiet lückenlos zu untersuchen. Grundlage für die Interpolation sind Daten der jeweiligen Fundorte (GPS-Punkte). Wie bereits in den vorangegangenen Kapiteln wird als Merkmalsanteil der gemittelte prozentuale Anteil an St. rubicundus Merkmalen der Individuen jedes GPS-Punktes definiert. Durch Interpolation wird flächendeckend der durchschnittliche Merkmalsanteil der Individuen im Gebiet des Tomaros errechnet (Abb. 54) 
Um die Ansicht noch zu verbessern, wird eine bilineare Filterung (oder bilineare Interpolation) durchgeführt, um die Zwischenwerte innerhalb des Rasters zu bestimmen (Abb. 55). Die Oberfläche besteht nun aus Zellen mit interpolierten Merkmalsanteilen.

Die errechneten Merkmalsanteile werden zwölf Gruppen zugeordnet und mit einem Farbcode, wie er auch schon bei den Hybridindices verwendet wurde, gekennzeichnet (Tab. 14). In rot bzw. blau sind interpolierte Rasterzellen die durchschnittliche Merkmalsanteile zwischen $0 \%$ und 2,5\% bzw. 97,5\% und 100\% darstellen, was St. clavatus (rot) bzw. St. rubicundus (blau) der Vergleichpopulationen entspricht. Den anderen Farben sind dementsprechend Merkmalsanteile zugeordnet, die - in entsprechender Abstufung - eine Nähe zu St. clavatus bzw. St. rubicundus ausweisen.

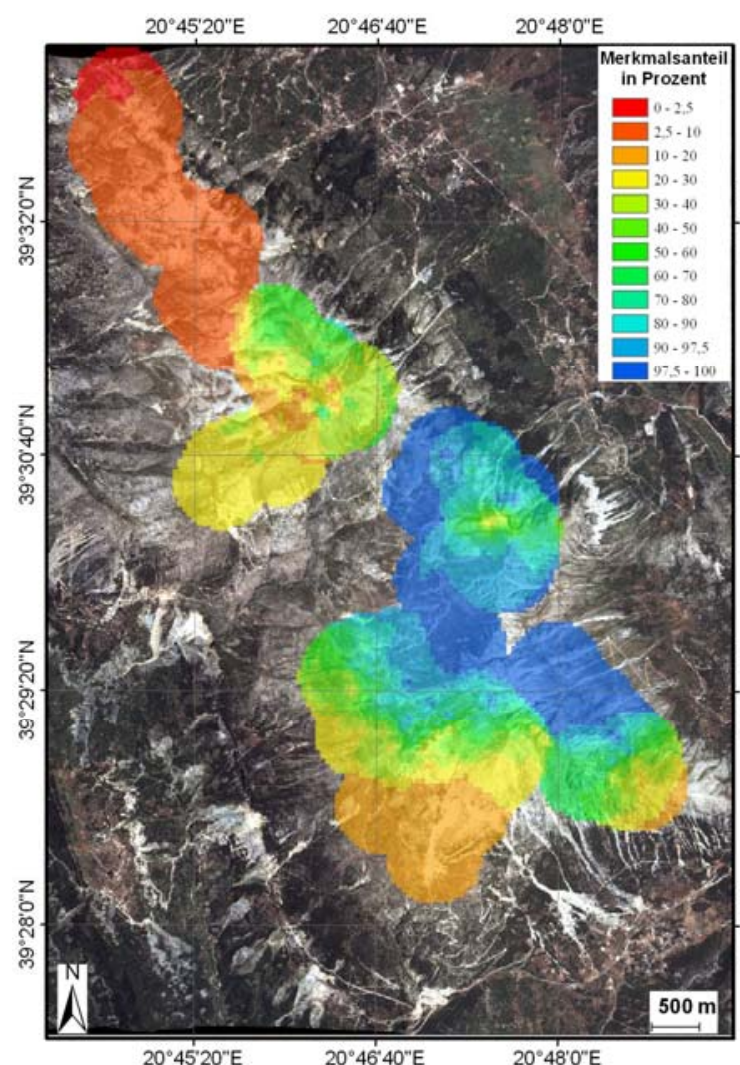

$20^{\circ} 45^{\prime} 20^{\prime \prime} \mathrm{E}$
Abb. 54 Rasterstruktur der interpolierten Merkmalsverteilung.

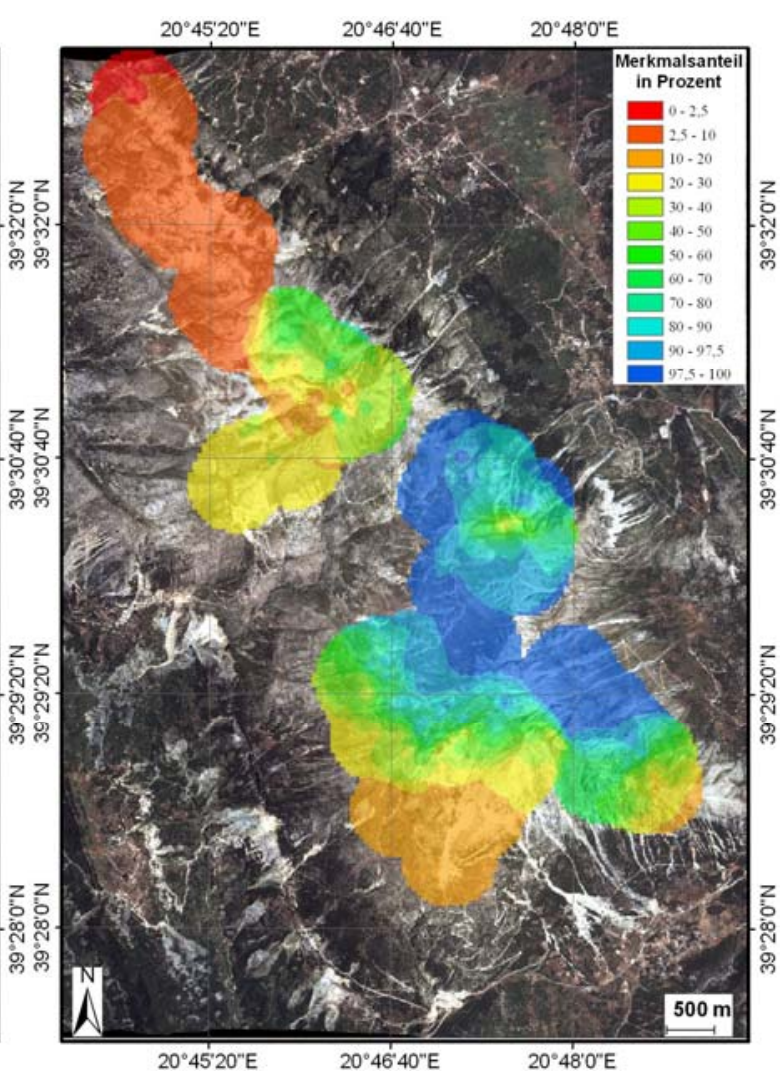

Abb. 55 interpolierte Merkmalsverteilung nach Glättung (bilineare Interpolation). 


\subsubsection{Untersuchung des räumlichen Musters der Merkmalsverteilung auf dem Tomaros}

Basierend auf den Rasteroberflächen aus Kapitel 3.1.7. wird die Verteilung der durchschnittlichen Merkmalsanteile auf dem Tomaros näher beschrieben.

Auf dem Nord-Tomaros findet man ganz im Norden ein kleines Gebiet, in dem den Individuen interpolierte Merkmalsanteile zwischen 0\% und 0,25 \% zuzuordnen sind. Somit befinden sich in diesem Bereich Individuen, die auf Basis des Vorderflügelmerkmals der St. clavatus-Referenzgruppe entsprechen. Es folgt in südlicher Richtung ein weiteres Gebiet, in dem die Individuen zum größten Teil noch der St. clavatus-Referenzgruppe entsprechen aber schon leichte Unterschiede zu dieser aufweisen (interpolierte Merkmalsanteile 0,26\% und 10\%). Dieses Gebiet erstreckt sich über zwei Drittel des Bergrückens. Im südlichen Teil des Nord-Tomaros sind an den östlichen Hängen der beiden vorderen kleinen Gipfel und im Bereich des Hauptgipfels, Gebiete, in denen die Individuen intermediäre Merkmalsanteile aufweisen. Auf den Gipfeln liegt der interpolierte Merkmalsanteil der Individuen zwischen 95\% und 99\%, somit zeigen diese Individuen große Ähnlichkeit zur Art St. rubicundus. Östlich dieser Gipfel erstreckt sich ein Gebiet, in dem der interpolierte Merkmalsanteil der Gruppe IV entspricht. Hier besitzen die Tiere Merkmalsanteile zwischen $20 \%$ und $30 \%$ und ähneln somit mehr der Art St. clavatus. Unterbrochen wird dieses von einer Zone in der die Merkmalsanteile der Individuen eher in den intermediären Bereichen liegen (zwischen $40 \%$ und 60\%).

Der Südtomaros lässt sich auf Grundlage der Merkmalsverteilung wie folgt einteilen: Auf dem Nord- und Osthang liegt der interpolierte Merkmalsanteil der Individuen im Bereich von St. rubicundus. Auf dem Süd- und Westhang hingegen sind Merkmalsanteile zwischen $10 \%$ und $20 \% \mathrm{zu}$ finden. Die hier vorkommenden Individuen weisen demnach größere Gemeinsamkeiten mit St. clavatus auf. Eine kleine Zone in der den Individuen intermediäre Merkmalsanteile zuzuordnen sind, ist im nördlichen Teil des Nordhanges zu finden. Ferner erstreckt sich ein langgezogenes Gebiet, in dem der Merkmalsanteil im intermediären Bereich liegt, zwischen den St. clavatus- und St. rubicundus-Gebieten von der Kante des Westhanges in Richtung Süden über den Sattel des Berges bis über den Osthang. 


\subsubsection{Bestimmung der Kontaktzonen}

Durch Interpolation können die Kontaktzonen beider Arten bestimmt werden. Also die Gebiete, in denen es am häufigsten zur Hybridisierung kommt.

Durch distanzbasierte Interpolation kann mathematisch bestimmt werden, wo Kontaktzonen zwischen den beiden Arten zu finden sind. Genau an der Grenze (Abb. 56) zwischen den Rasterzellen mit Merkmalsanteilen 49\%-51\% ist die Kontaktzonen zwischen beiden Arten definiert worden (Kapitel 2.7.3).

Drei Kontaktzonen sind mit Hilfe der Interpolation bestimmt worden (Abb. 56). Kontaktzone I erstreckt sich von Nordwesten des Südtomaros über den Sattel bis hin zum südlichen Teil des Osthanges und ist ca. 4,8 Kilometer lang. Kontaktzone II (ca. 350 m Länge) ist an der nördlichen Spitze des Südtomaros zu finden. Auf dem Nordtomaros befindet sich die dritte Kontaktzone und zwar im südlichen Teil dieses Berges etwas unterhalb der kleinen Gipfel. Sie besteht aus drei Abschnitten die zusammen 1,6 Kilometer lang sind (Abb. 56).

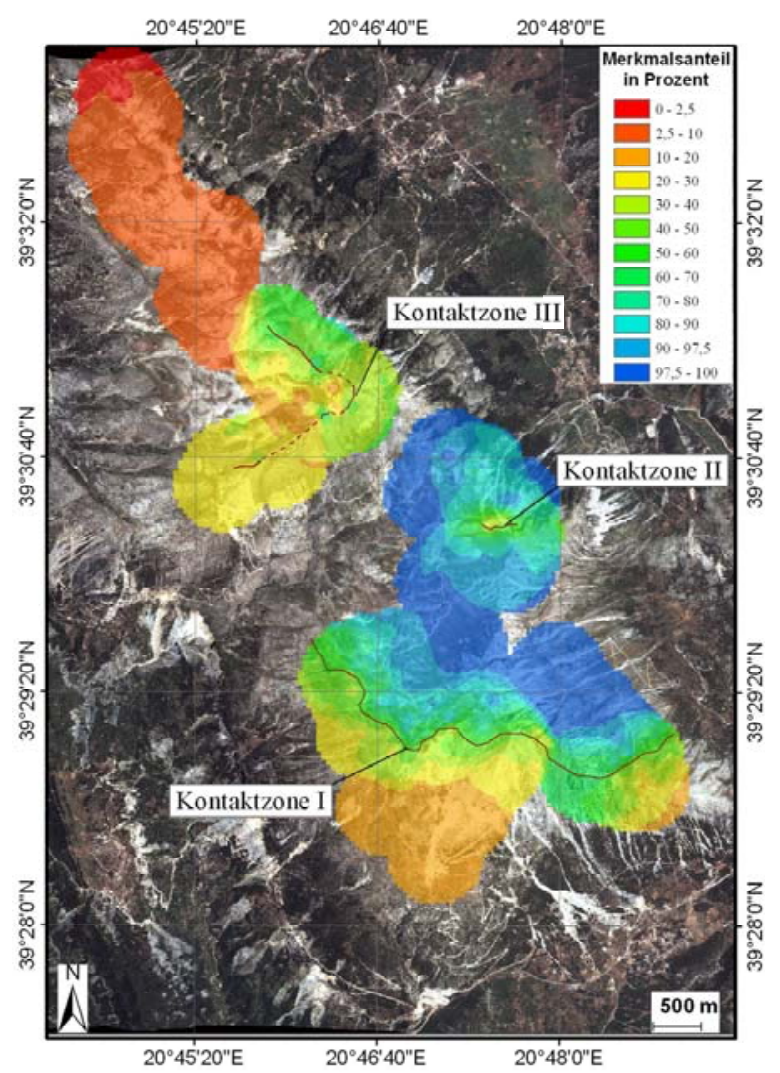

Abb. 56 Die Lage der drei Kontaktzonen zwischen St. rubicundus und St. clavatus auf dem Tomaros. 
Auf Grundlage dieser drei Kontaktzonen können im folgenden Kapitel die jeweiligen Merkmalskline berechnet werden.

\subsubsection{Kline des Vorderflügelmerkmals}

Wie in Material und Methoden beschrieben, sind Kline Merkmalsveränderungen entlang einer geographischen Grenze. Als geographische Grenze dienen in diesem Fall die Habitatsgrenzen der untersuchten Populationen auf dem Tomaros. Hierzu wurden ausgehend von den Zentren der Kontaktzonen die Merkmalsveränderungen an den einzelnen Fundorten auf dem Tomaros untersucht (Kapitel 2.7.4). Da aber die Kontaktzonen eine geschwungene Form aufweisen, wurde wie folgt vorgegangen. Zur Berechnung der Klinen wurde das Programm ArcGIS verwendet, das zum einen die kürzeste Entfernung der Fundorte zur Kontaktzone und zum anderen die Himmelsrichtung (durch einen Richtungswinkel) zur Kontaktzone ermittelt. Die Entfernungen von Fundorten, die südlich und westlich der Kontaktzonen liegen, werden mit positiven und solche, die nördlich und östlich liegen, mit einem negativen Vorzeichen versehen. Somit fließt die Himmelsrichtung der Fundorte im Bezug zur Kontaktzone mit ein.

\subsubsection{Kline ausgehend von Kontaktzone I}

Insgesamt 84 Fundorte sind in unmittelbarer Nähe zu Kontaktzone I zu finden. Trägt man die durchschnittlichen Merkmalsanteile der Individuen dieser Fundorte gegen die Entfernung zur Kontaktzone I auf, so erhält man eine sigmoide Kurve (Abb. 57).

Fundorte an denen die Individuen intermediäre Merkmalsanteile (zwischen 40\% und 60\%) aufweisen, sind auf beiden Seiten bis zu einem Abstand von 100 m zur Kontaktzone I zu finden. Die Zone mit Fundorten, an denen die Merkmalsanteile der Individuen im intermediären Bereich liegen, ist also maximal $200 \mathrm{~m}$ breit, aber an vielen Stellen weitaus schmaler. Ausgehend von Kontaktzone I in nördlicher und östlicher Richtung werden die durchschnittlichen Merkmalsanteile der Individuen an diesen Standpunkten kontinuierlich größer und ab einer Entfernung von ca. $260 \mathrm{~m}$ liegen diese konstant bei einem mittleren Merkmalsanteil von $95 \%$ bis $100 \%$. Mit anderen Worten: die hier vorkommenden Individuen entsprechen hinsichtlich der Flügelmerkmale nahezu ausnahmslos der St. rubicundusReferenzgruppe. In der entgegen gesetzten Richtung, also in südlicher und westlicher Richtung werden die Merkmalsanteile an den Fundorten bis zu einer Entfernung von ca. $250 \mathrm{~m}$ zur Kontaktzone I kontinuierlich kleiner und pegeln sich dann zwischen $21 \%$ und 
$15 \%$ Merkmalsanteil ein. Das bedeutet, dass die hier lebenden Tiere bzgl. der Flügelmorphologie nicht vollständig denen der St. clavatus-Referenzgruppe entsprechen. Diese Unsymmetrie der Kline wird später noch zu diskutieren sein.

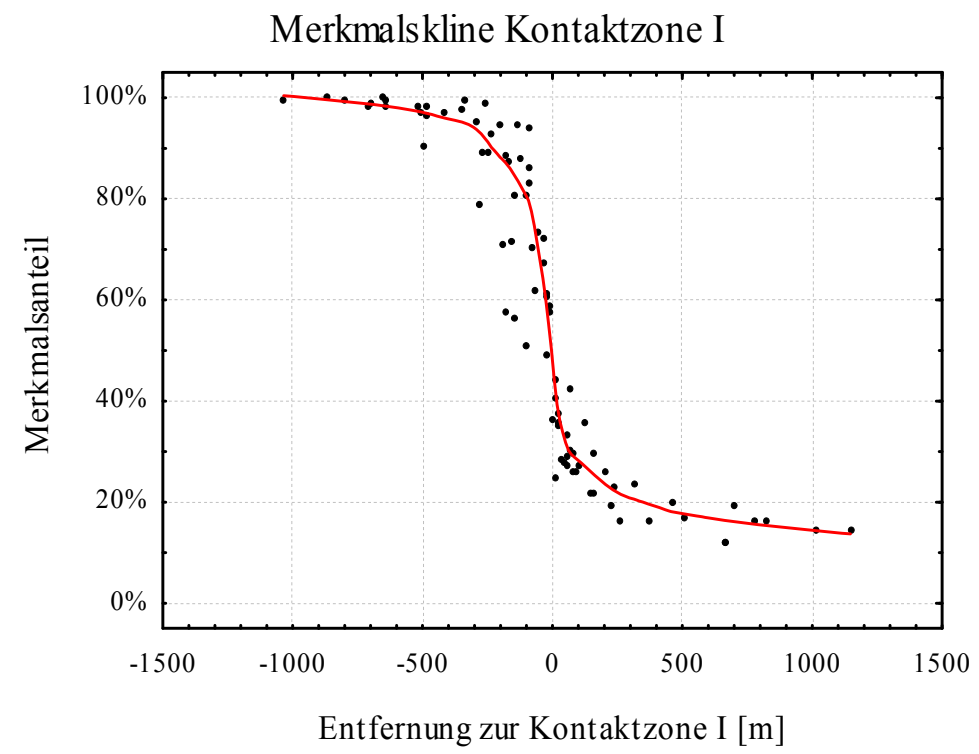

Abb. 57 Vorderflügel-Merkmalskline im Bereich der Kontaktzone I. Lowess-Glättung (-) h=0,25

\subsubsection{Kline ausgehend von Kontaktzone II}

Anders als Kontaktzone I, liegt die Kontaktzone II in mitten eines Gebietes in dem hauptsächlich Individuen zu finden sind, die Vorderflügelmerkmale der Art St. rubicundus aufweisen. Sie befindet daher am Rand einer kleinen St. clavatus-Population inmitten von St. rubicundus.

In unmittelbarer Nähe zur Kontaktzone II liegen 20 Fundorte (Abb. 58). Aber anders als bei der Betrachtung der Kontaktzone I, findet man hier sehr wenige Fundorte, an denen die Merkmalsanteile unter 50\%, also auf der Seite der Art St. clavatus, liegen. Ebenso selten sind Fundorte mit ausgesprochen intermediären Tieren. Anders als die vorige ist Kontaktzone II umrahmt von Fundorten mit Individuen mit St. rubicundus-Charakter: Sowohl auf der südlichen als auch auf der westlichen Seite der Kontaktzone findet man zum größten Teil Fundorte, an denen die Individuen durchschnittliche Merkmalsanteile zwischen 90\% und $100 \%$ besitzen. Hier sind also hauptsächlich Tiere zu finden, die ähnlich der Art St. rubicundus sind oder dieser Referenzgruppe voll entsprechen. 


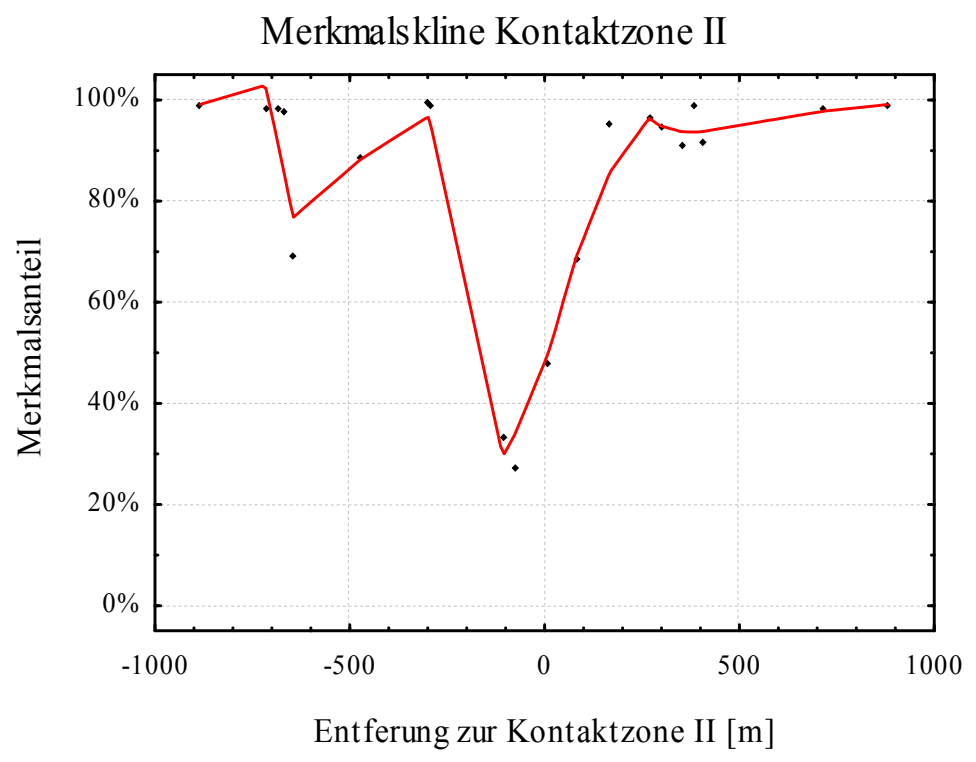

Abb. 58 Vorderflügel-Merkmalskline im Bereich der Kontaktzone II. Lowess-Glättung (-) h=0,25.

\subsubsection{Kline ausgehend von Kontaktzone III}

Der Kline im Bereich der Kontaktzone III beschreibt eine asymmetrische Kurve, die zunächst steil in Richtung Norden abfällt und sich dann bei einem konstanten Wertebereich stabilisiert (Abb. 59). Südlich der Kontaktzone III befindet sich ein steiler Hang der nicht begehbar ist. Aus diesem Grund konnten nur zwei GPS-Punkte gesetzt werden, so dass Daten aus diesem Bereich fehlen.

$\mathrm{Zu}$ den Fundorten ist im einzelnen Folgendes zu sagen: Auf der südlichen und westlichen Seite findet man im Abstand von $10 \mathrm{~m}$ bis $30 \mathrm{~m}$ zwei Fundorte, an denen die Merkmalsanteile der Individuen der einzelnen Fundorte zwischen 70\% und 90\% liegen. An drei weiteren Fundorten in unmittelbarer Nähe zur Kontaktzone $(6 \mathrm{~m}$ bis $16 \mathrm{~m})$ sind Individuen mit jeweils durchschnittlichen Merkmalsanteilen im intermediären Bereich, von $60 \%$ bis $40 \%$ zu finden. In nördlicher und östlicher Richtung, bis zu einer Entfernung von 270 m, verringert sich der durchschnittliche Merkmalsanteil der Individuen an den einzelnen Fundorten stetig von weniger als $40 \%$ bis auf ca. $10 \%$. Ab dieser Entfernung verändern sich die Merkmalsanteile an den Fundorten kaum und bewegen sich im Bereich zwischen 4\% und 8\% bis zu einer Entfernung von 2600m zur Kontaktzone III. Die Heuschrecken in diesem Gebiet haben also hauptsächlich St. clavatus Merkmale, unterscheiden sich aber doch noch etwas von der St. clavatus Referenzgruppe. Einzig an den weitest entfernten Fundorten besitzen die Individuen durchschnittliche Merkmalsanteile von 2\% und entsprechen hier somit der Art St. clavatus. 


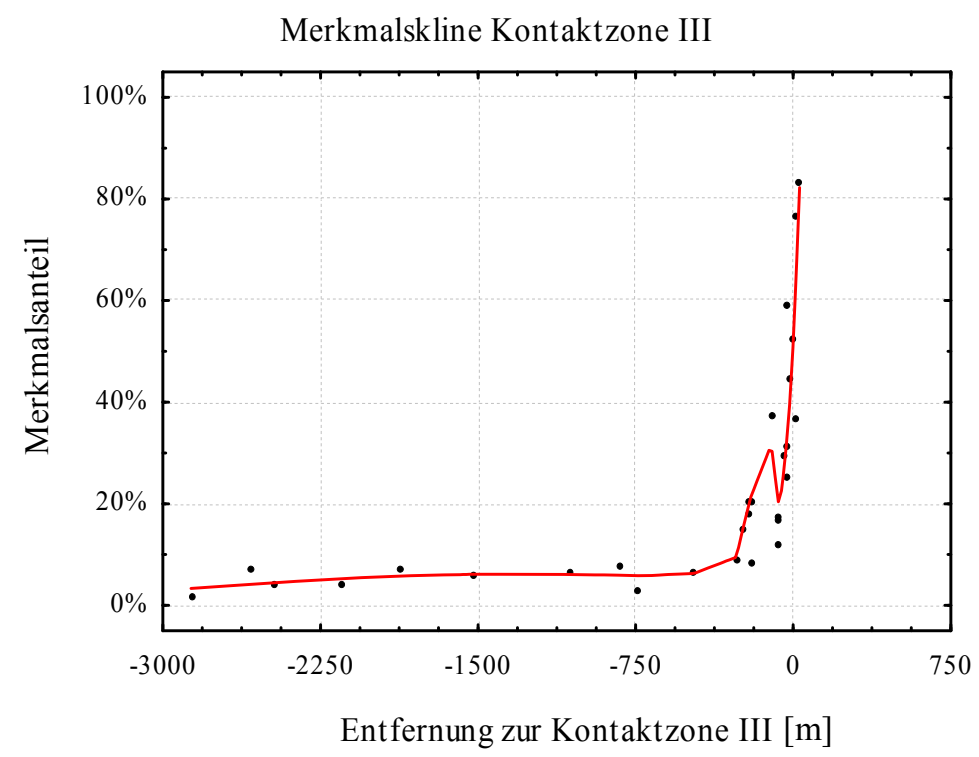

Abb. 59 Vorderflügel-Merkmalskline im Bereich der Kontaktzone III. Lowess-Glättung (-) h=0,25

\section{Zusammenfassung}

Im Bereich der drei Kontaktzonen sind verschiedene Klineverläufe beschrieben worden. Eine sigmoide Kurve (Kontaktzone I) eine asymetrische Kurve (Kontakzone III) und eine sehr heterogene (Kontaktzone III).

Trotz der unterschiedlichen Form der Kline gibt es ausgehend vom Zentrum der Kontaktzonen auch einige Gemeinsamkeiten: Bis zu einer Entfernung von ca. $200 \mathrm{~m}$ ist eine rasche Zunahme (Seite der Art St. rubicundus) bzw. Abnahme (St. clavatus-Seite) der Merkmalsanteile zu beobachten. Ab dieser Entfernung verändern sich die Merkmalsanteile nur noch wenig und pendeln sich um einen bestimmten Wert ein (nur bei Kontaktzone I und II). Die Merkmalsanteile auf der St. rubicundus-Seite erreichen Werte nahe 100\%, hingegen pegeln sich die Merkmalsanteile auf der St. clavatus-Seite zwischen $20 \%$ und $15 \%$ bei Kontaktzone I, und zwischen $8 \%$ und 1,6\%, bei Kontaktzone III, ein.

Eine etwas andere Merkmalsentwicklung ist im Bereich der Kontaktzone II zu beobachten. Die durchschnittlichen Merkmalsanteile der Individuen an den einzelnen Fundorten, die der Art St. rubicundus ähnlich sind, pegeln sich nahe 100\% ein. Aber an den Fundorten an denen Individuen mehrheitlich St. clavatus Merkmale zugeordnet werden, sind durchschnittliche Merkmalsanteile zwischen 20\% und 25\% ermittelt worden. 


\subsection{Morphometrische Vermessung der Antennen}

Bei der morphometrischen Vermessung der Antennen wurde die breiteste Stelle innerhalb des Bereichs der letzten fünf Antennensegmente sowie die Breite des Antennensegmentes gemessen, das auf die Flagellomersegmente folgt (Abb. 21). Der Quotient aus beiden Werten dient als - relatives - Maß für die Antennenbreite (siehe Material und Methoden, Kap. 2.5). Zur Vermessung wurden 1116 (739ð; 377ㅇ) Individuen herangezogen. Die Tiere stammen sowohl von Standorten, an denen ausschließlich eine der beiden Arten vorkommt, als auch vom Tomaros. In den nächsten Kapiteln wird zuerst auf den zwischenartlichen Unterschied zwischen St. clavatus und St. rubicundus eingegangen. Auf Grundlage dieser Daten werden dann die Tiere des Tomaros morphologisch vermessen und eingeordnet.

\subsubsection{Zwischenartlicher Vergleich von St. clavatus und St. rubicundus}

Für den zwischenartlichen Vergleich wurden Tiere herangezogen, die ausschließlich aus allopatrischen Populationen stammen, die keinen Kontakt zur jeweils anderen Art haben. Bei St. clavatus waren dies Tiere vom Xerovouni. Im Falle von St. rubicundus wurden, wie bei der flügelmorphometischen Vermessung, Tiere von mehreren Standorten (Slowenien, Vernon, Mitsikeli, Metsovo und Peloponnes) für den Vergleich herangezogen. Anders als bei der flügelmorphometrischen Vermessung gibt es hier keine signifikanten Unterschiede zwischen den slowenischen und den griechischen Populationen.

Die letzten Antennalsegmente von St. rubicundus-Männchen $(\mathrm{N}=54)$ messen an der breitesten Stelle durchschnittlich 0,32 mm $(\sigma \pm 0,022)$. Das basale Segment ist durchschnittlich $0,28 \mathrm{~mm}(\sigma \pm 0,018)$ breit. Das Verhältnis zwischen den beiden Messgrößen beträgt im Mittel 1,15 $(\sigma \pm 0,09)$ und variiert zwischen 0,95 und 1,41. Die Antennen der Weibchen $(\mathrm{N}=22)$ sind bei St. rubicundus etwas schmaler als die der Männchen. Hier wurde im Bereich der oberen Antennalsegmente eine maximale Breite von durchschnittlich 0,28 mm ( $\sigma$ $\pm 0,013)$ gemessen. An der Basis ist die Antenne 0,26 $\mathrm{mm}(\sigma \pm 0,017)$ breit und somit ergibt dies ein Verhältnis von durchschnittlich 1,05 $(\sigma \pm 0,065)$.

Bei den 36 Männchen der Art St. clavatus, die vom Xerovouni stammen, wurde eine Breite der letzten Segmente von 0,75 mm $(\sigma \pm 0,061)$ gemessen. Im Bereich des basalen Messbe- 
reiches sind die Antennen dieser Tiere durchschnittlich 0,28 $(\sigma \pm 0,021) \mathrm{mm}$ breit. Das Verhältnis zwischen diesen beiden Werten beträgt durchschnittlich 2,64 $(\sigma \pm 0,23)$ und variiert zwischen 2,13 und 3,14. Des Weiteren wurden zehn Weibchen der Art St. clavatus vermessen (Abb. 60 rechts); die maximale Breite der letzten Antennensegmente beträgt durchschnittlich 0,44 mm ( $\sigma \pm 0,022)$, und an der Basis haben die Antennen der Weibchen eine Breite von durchschnittlich 0,27 mm $(\sigma \pm 0,011)$. Das Verhältnis beider Werte liegt im Mittel bei 1,59 $(\sigma \pm 0,07)$. Somit sind die Antennen der St. clavatus-Weibchen im oberen Bereich durchschnittlich 0,31 mm schmaler als die der Männchen. An der Basis der Antennen ist der Unterschied nicht sehr groß und liegt lediglich bei $0,1 \mathrm{~mm}$.

Zusammenfassung (Abb. 60).: Die Antennen der Arten St. rubicundus und St. clavatus unterscheiden sich also nicht in der Breite der basalen, sondern in der Breite der letzten Antennensegmente. Hier unterschieden sich die Männchen beider Arten um 0,43 mm. Bei den Weibchen ist der Unterschied zwischen den Arten kleiner und liegt bei 0,16 mm. Diese Unterschiede machen sich auch beim Verhältnis beider Werte bemerkbar. Für die weitere Berechnung wird das Verhältnis zwischen der Antennenbreite im Bereich der letzten Segmente gegen die Breite des basalen Antennensegmentes, als Maß für die Antennenbreite benutzt. Männchen wie Weibchen unterscheiden sich jeweils signifikant voneinander.
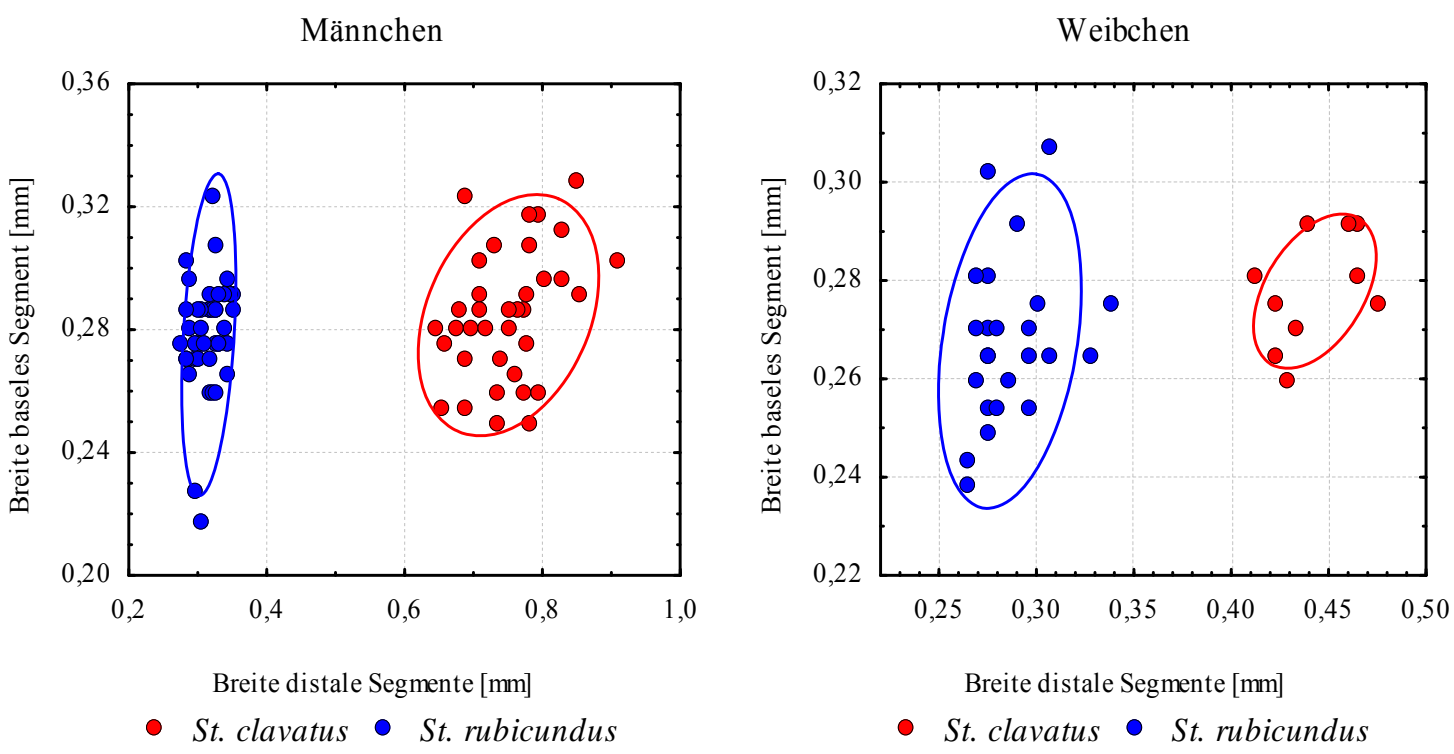

Abb. 60 Morphometrischer Vergleich zwischen Männchen (links) und Weibchen (rechts) der Arten St. clavatus (rot) und St. rubicundus (blau) hinsichtlich der Breite der Antennenglieder. 


\subsubsection{Antennenmorphologie der Individuen des Tomarosgebirges}

Aus dem Kapiteln über die Vorderflügelmorphometrie geht hervor, dass es auf dem Tomaros Vertreter beider Arten, aber auch deren Zwischenformen gibt. Dies ergibt sich auch aus den Daten zur Antennenmorphologie (Abb. 61 links und rechts). Einige Tiere entsprechen den Referenzarten, und ein beträchtlicher Anteil der Tomaros-Tiere zeigt Merkmale, die zwischen den beiden Arten liegen. Dies ist bei den Männchen in Abb. 61 (links) aber auch bei den Weibchen des Tomaros zu beobachten (Abb. 35 rechts). Somit ist auch hier eine Einteilung der Individuen in ein Hybridindex- und Merkmalsanteils-System möglich, um die Individuen des Tomaros zu klassifizieren.
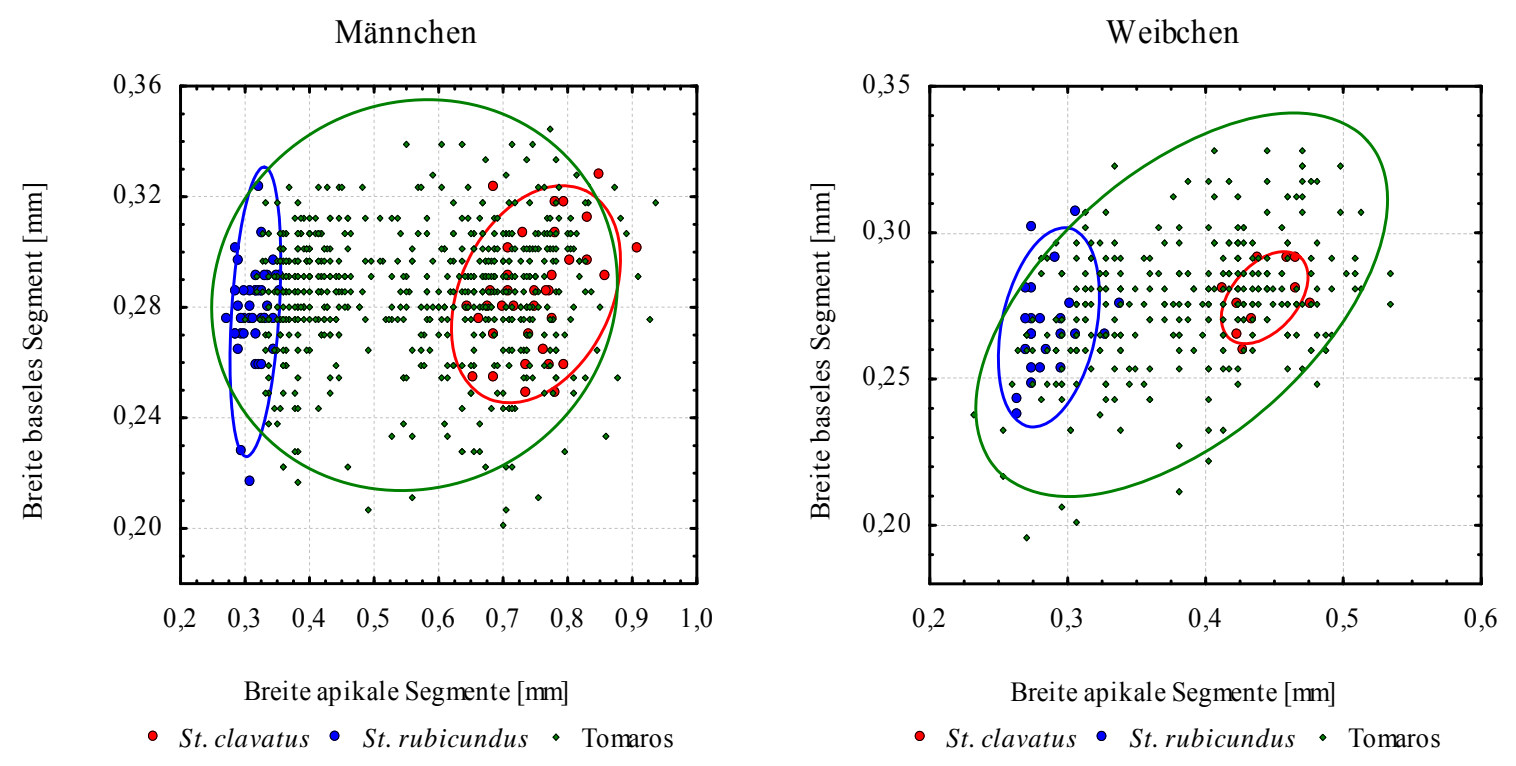

Abb. 61 Morphometrischer Vergleich zwischen Männchen (links) und Weibchen (rechts) der allopatrischen Populationen von St. clavatus (rot), St. rubicundus (blau) und Individuen des Tomarosmassives (grün).

Bei der Klassifizierung der Individuen auf Basis der Antennenmorphologie wird ähnlich wie bei der Klassifizierung auf Grundlage der Vorderflügelmorphometrie vorgegangen. Tiere, bei denen die relative Antennenbreite der letzten Segmente innerhalb der Varianz der Referenzgruppen von St. rubicundus oder St. clavatus liegt, werden einer dieser Arten zugeteilt. Sie erhalten den Hybridindex 1, wenn sie St. clavatus entsprechen oder den Hybridindex 12, wenn sie auf Grundlage der relativen Antennenbreite der Art St. rubicundus gleichen. Einen Hybridindex von 2 bis 11 erhalten jene Individuen, die Antennenmerkmale aufweisen, welche zwischen den beiden Arten liegen. 
Bei der Benennung der Merkmalsanteile wird nach dem gleichen Prinzip wie bei der Flügelmorphologie vorgegangen: Jedem Individuum wird jeweils ein Merkmalsanteil der Art St. rubicundus und der Art St. clavatus zugeordnet. Ein Individuum mit einer Antennenbreite innerhalb der Varianz der St. rubicundus-Referenzgruppe besitzt somit einen Merkmalsanteil von 100\% St. rubicundus und 0\% St. clavatus; bei einem Individuum mit einer relativen Antennenbreite innerhalb der Varianz der St. clavatus Referenzgruppe ergibt sich der reziproke Fall. Intermediäre Individuen haben z. B. einen Merkmalsanteil von 35\% St. clavatus und $65 \%$ St. rubicundus. Zur Charakterisierung wird auch hier nur einer der beiden Werte, nämlich der Merkmalsanteil von St. rubicundus benutzt, in diesem Falle also $65 \%$; siehe im übrigen Kapitel 3.1.5.2.

Tab. 18 Einteilung der Männchen (links) und Weibchen (rechts) auf Grundlage der Antennenmorphologie in Merkmalsanteile und Hybridindices (HI)

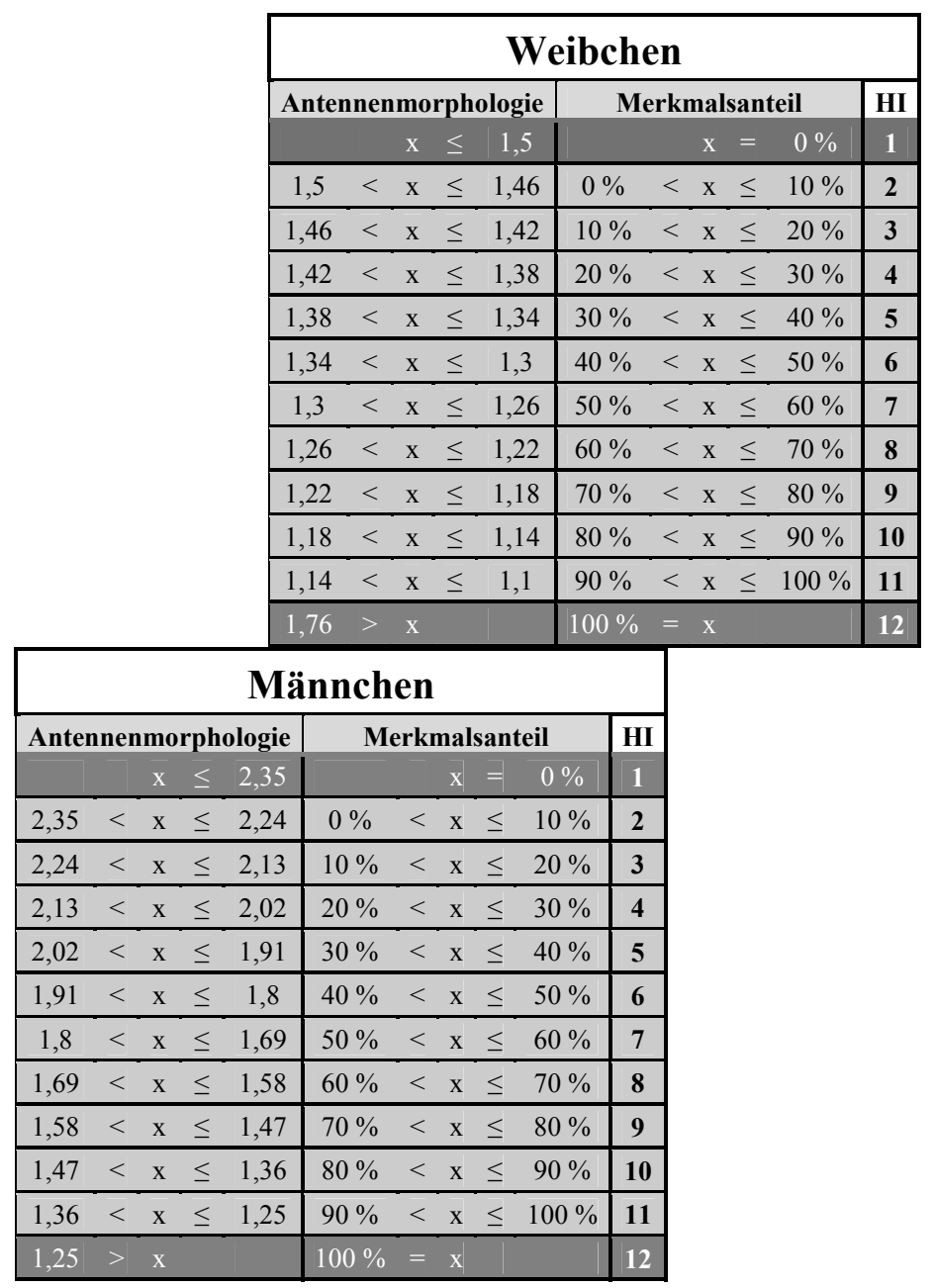

Auf diese Art und Weise werden nun den Tieren des Tomaros Hybridindices und Merkmalsanteile zugeteilt. Auf Grundlage ihrer Fundorte, die mit GPS markiert worden sind, werden die Individuen zu Gruppen zusammengefasst. 


\subsubsection{Charakterisierung der Tomaros-Populationen von St. clavatus, St. rubicundus und deren Hybriden aufgrund der Antennenmorphologie}

Zur detaillierten Charakterisierung der Individuen des Tomarosgebirges wurden die Antennen von 1061 (705 ; 356ㅇ) vermessen und ihnen Hybridindices sowie Merkmalsanteile zugeordnet. Hierbei ergibt sich: Dem Hybridindex 1 werden 34,4 \% (365 Tiere) der Individuen zugeordnet. Somit entsprechen sehr viele der Tomaros-Tiere gemäß ihrem Antennenmerkmal der St. clavatus-Referenzgruppe. Jeweils 7\% der Individuen ( $\mathrm{N}=74)$ werden den Hybridindices 2 und 3 zugeteilt; diese Individuen zeigen noch eine gewisse Ähnlichkeit zur St. clavatus-Referenzgruppe. Tiere mit Hybridindex $4-8$ sind weniger stark vertreten: Index 4 (43 Individuen =4,1\%), Index 5 (32 Individuen =3,0\%), Index 6 (34 Individuen $=3,3 \%)$, Index 7 (30 Individuen $=2,9 \%)$ und Hybridindex 8 (36 Individuen =3,8 \%). Zum Hybridindex 9 lassen sich 55 (5,8\%) Tiere und dem Index 9 - 81 Individuen (7,9\%) zuordnen. Etwas stärker vertreten sind Individuen die der auf Basis des Antennenmerkmals der St. rubicundus Referenzgruppe stark ähneln (Index 11) oder dieser völlig entsprechen (Index 12). Diesen beiden lassen sich 109 Individuen (10,6 \%) dem Hybridindex 11 und 97 Individuen (10,3\%) dem Index 12 zuordnen. Der durchschnittliche Merkmalsanteil der Individuen des Tomaros liegt somit bei 40,4 \% $(\sigma \pm 40,7)$.

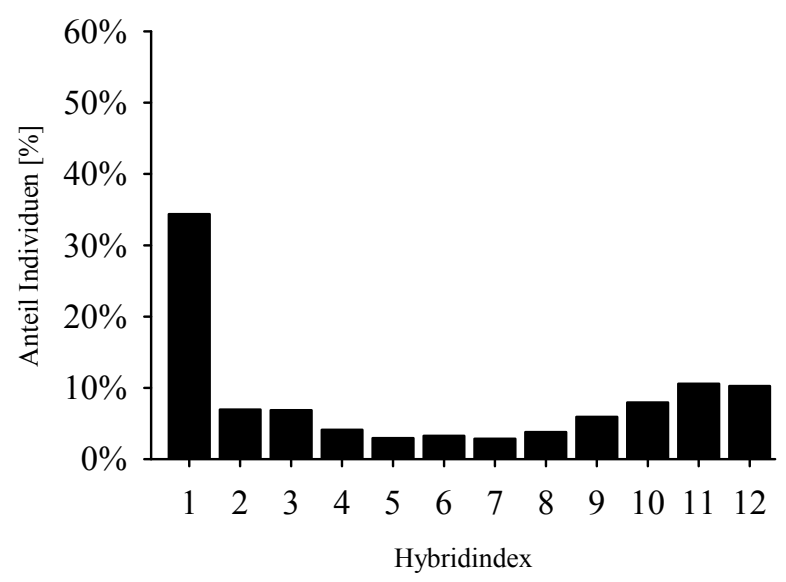

Abb. 62 Gesamtverteilung der Hybridindices (Vorderflügelmerkmal) auf dem gesamten Tomarosmassiv

Auch bei der Untersuchung der Antennen werden die Individuen ihren Fundorten (GPSPunkte) zugeordnet. Für die Antennenvermessung wurden Tiere von 83 GPS-Punkten des Tomarosgebirges verwendet. Und auch hier wurde der Tomaros in jene drei Gebiete einge- 
teilt, die auch bei der Flügelvermessung benannt worden sind (Abb. 44): Nordtomaros (20 GPS-Punkte), Südtomaros (43 GPS-Punkte) und Osthanges des Südtomaros (20 GPSPunkte).

\subsubsection{Merkmalsverteilung auf dem Tomaros-Nordmassiv}

Es wurden von insgesamt 189 Individuen, die an 20 GPS-Punkten gesammelt worden waren, die Antennen vermessen und jedes dieser Individuen mit einem Hybridindex versehen.

Allgemeine Übersicht: Auf dem Nordtomaros dominieren Individuen hauptsächlich des Hybridindex 1 (Abb. 63): Es entsprechen 103 (54,5\%) Individuen, also der Großteil, der St. clavatus-Referenzgruppe. Weit aus weniger Individuen können dem Hybridindex 2 (16 Tiere $=8,5 \%)$ und dem Index $3(15$ Tiere $=7,9 \%)$ zugeordnet werden. Des Weiteren wurden sehr wenige Individuen mit einen Hybridindex von 4 bis 8 gefunden; es waren nur zwischen drei und fünf (1,6\%-2,6\%). Einen Hybridindex von 9 besitzen sechs (3,2\%) und einen Index von 10 - fünf (2,6\%) Individuen. Außerdem entsprechen 17 (9,0\%) Individuen dem Hybridindex 11 und acht (4,2\%) Tiere können Hybridindex 12 zugeordnet werden und entsprechen so der St. rubicundus-Referenzgruppe.

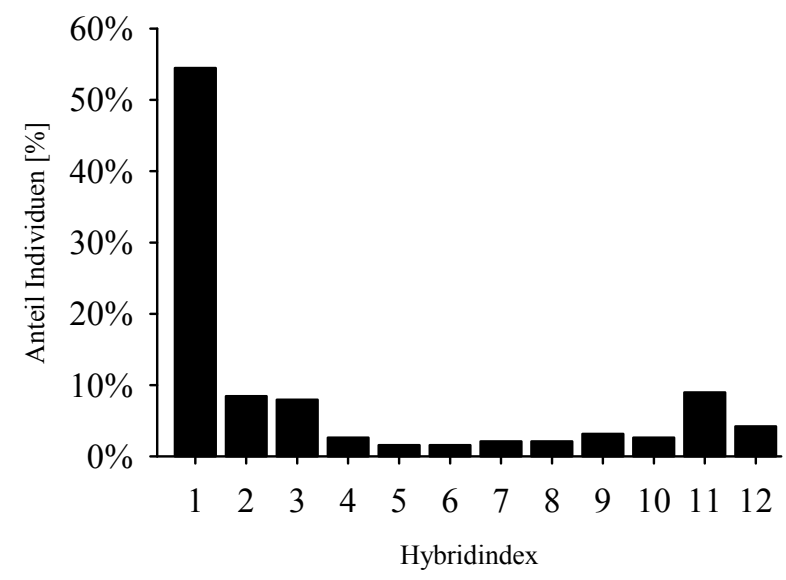

Abb. 63 Gesamtverteilung der Hybridindices (Antennenmerkmal) auf dem Nord-Tomaros

Merkmalsverteilung im Detail: Es werden nun die Merkmalsverteilung der 20 verwendeten Fundorte des Nordtomaros genauer von Norden nach Süden beschrieben. Wie in Abb. 64 gezeigt, werden die durchschnittlichen Merkmalsanteile in Form eines Kreisdiagramms und die Hybridindices mit Hilfe eines gestapelten Balkendiagramms (Abb. 65) für jeden GPS-Punkt (Fundort) graphisch dargestellt. Diese Darstellungsweise entspricht der für die Flügelmorphologie, siehe hierzu Kapitel 3.1.6.1 bis 0. 


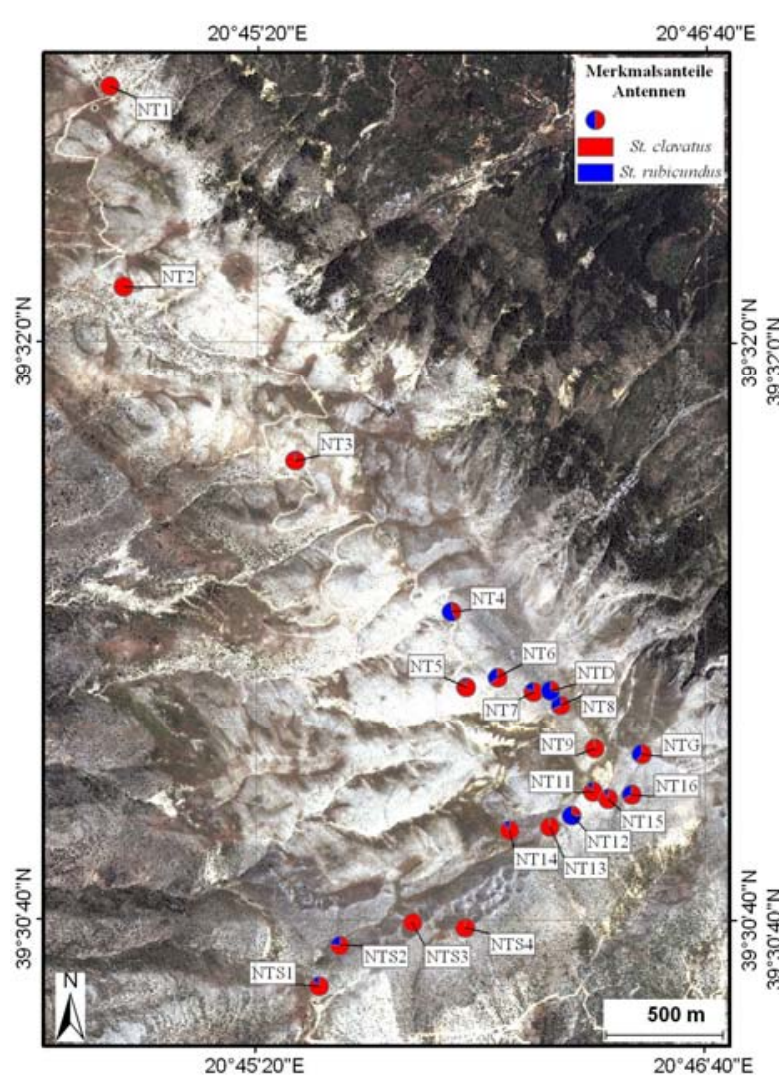

Abb. 64 Verteilung der durchschnittlichen Merkmalsanteile [\%] auf dem Nordtomaros

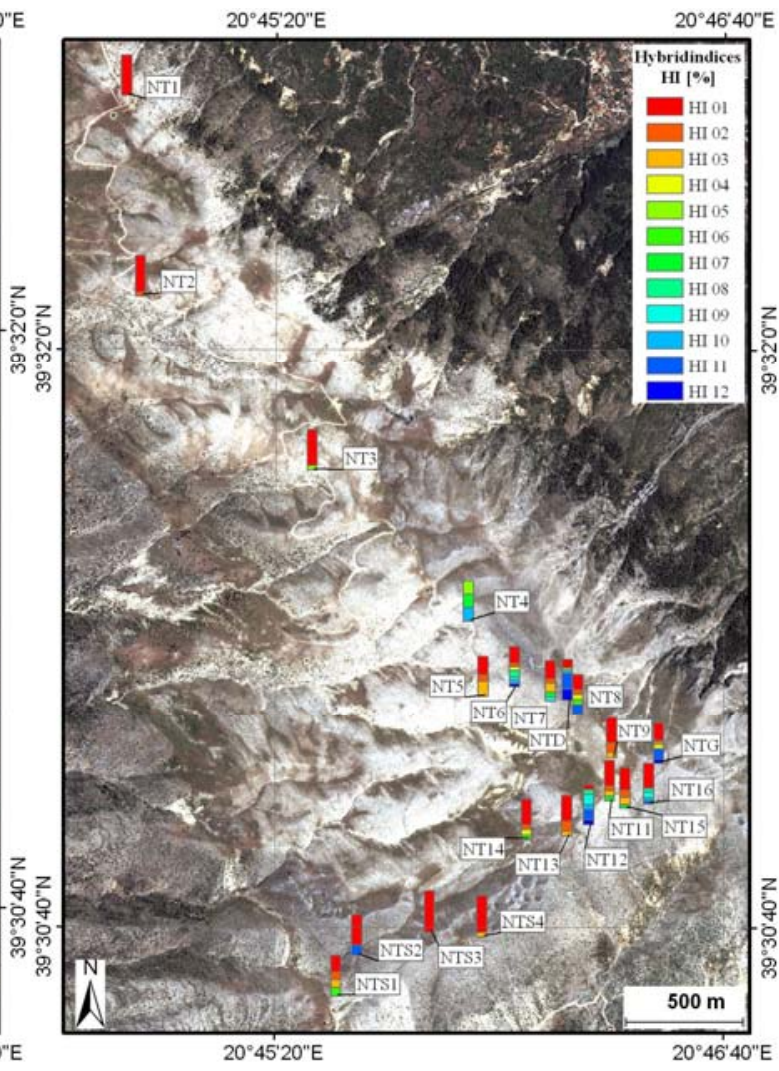

Abb. 65 Anteil der Individuen mit entsprechenden Hybridindices für jeden Fundort auf dem Nordtomaros

Von Norden über den Bergrücken des Nordmassives sind zunächst an drei Fundorten (Tab.

19) Tiere gefunden worden die zu einem sehr großen Teil der St. clavatus- Referenz-

Gruppe entsprechen. Der durchschnittliche Merkmalsanteil dieser drei Fundorte liegt bei $1,36 \%$ und variiert zwischen $0 \%$ und $4,04 \%$.

Tab. 19 Merkmalsverteilung der Fundorte (GPS) im nördlichen Teilgebiet des Nordtomaros

\begin{tabular}{|c|c|c|c|c|c|c|c|c|c|c|c|c|c|c|c|c|c|c|}
\hline \multicolumn{9}{|c|}{ Geographische Parameter } & \multicolumn{1}{|c|}{ Mybridindices } & \multicolumn{3}{c|}{ Merkmalsanteil } \\
\hline GPS & Längengrad & Breitengrad & Höhe & 1 & 2 & 3 & 4 & 5 & 6 & 7 & 8 & 9 & 10 & 11 & 12 & $\boldsymbol{\Sigma}$ & $\overline{\mathbf{x}}$ & $\sigma$ \\
\hline NT1 & $39^{\circ} 32^{\prime} 35,2^{\prime \prime} \mathrm{N}$ & $20^{\circ} 44^{\prime} 53,6 \mathrm{E} \mathrm{E}$ & $1281 \mathrm{~m}$ & 9 & - & - & - & - & - & - & - & - & - & - & - & $\mathbf{9}$ & $\mathbf{0 , 0 0 \%}$ & 0,0 \\
\hline NT2 & $39^{\circ} 32^{\prime} 07,6^{\prime \prime} \mathrm{N}$ & $20^{\circ} 44^{\prime} 56,1^{\prime \prime} \mathrm{E}$ & $1419 \mathrm{~m}$ & 8 & 1 & - & - & - & - & - & - & - & - & - & - & $\mathbf{9}$ & $\mathbf{0 , 0 6 \%}$ & 0,2 \\
\hline NT3 & $39^{\circ} 31^{\prime} 43,4^{\prime \prime} \mathrm{N}$ & $20^{\circ} 45^{\prime} 26,9^{\prime \prime} \mathrm{E}$ & $1516 \mathrm{~m}$ & 8 & - & - & - & - & - & - & - & - & - & - & - & $\mathbf{9}$ & $\mathbf{4 , 0 3 \%}$ & 12,1 \\
\hline
\end{tabular}

Im südlichen Teil des Nordtomaros befinden sich 17 Fundorte (Tab. 20), die eine sehr heterogene Merkmalsstruktur aufweisen. In diesem Teilgebiet wurden viele Individuen gefunden, die Antennen aufweisen, die der St. clavatus-Referenzgruppe entsprechen (Index 1) oder dieser sehr ähnlich sind (Index 2 und 3). Zudem findet man hier aber auch Individuen mit Merkmalen, die der Art St. rubicundus sehr ähnlich sind (10 und 11) oder dieser ganz entsprechen (Index 12), wobei der Anteil der Tiere mit einem Hybridindex 12 allerdings sehr klein ist. Aber auch Individuen mit intermediären Antennenmerkmalen (Index 4 
bis 9) sind in diesem Teilgebiet zu finden. Des Weiteren kommen bei sieben Fundorten Individuen mit Antennenmerkmalen im Bereich von St. clavatus zusammen mit Tieren, die Merkmale im Bereich der Art St. rubicundus besitzen, vor. Einzelne intermediäre (Index 6 -8) Individuen findet man zusätzlich hierzu an den Punkten NT4, NT7, NT11, NT15 und NT12 (Tab. 20). Die Merkmalsanteile dieses Teilgebietes liegen bei durchschnittlich $24,3 \%$ variieren aber zwischen $0 \%$ und $74,2 \%$.

Somit lässt sich das Tomaros-Nordmassiv in einen nördlichen und einen südlichen Bereich einteilen. Im nördlichen sind Individuen zu finden, die größtenteils der Art St. clavatus entsprechen. Im südlichen Teil findet man Tiere, die Merkmale im Bereich von St. clavatus, wenige intermediäre und wenige Tiere mit Merkmalen die der Art St. rubicundus entsprechen.

Tab. 20 Merkmalsverteilung der Fundorte (GPS) im südlichen Teilgebiet des Nordtomaros

\begin{tabular}{|c|c|c|c|c|c|c|c|c|c|c|c|c|c|c|c|c|c|c|}
\hline \multicolumn{4}{|c|}{ Geographische Parameter } & \multicolumn{13}{|c|}{ Hybridindices } & \multicolumn{2}{|c|}{ Merkmalsanteil } \\
\hline GPS & Längengrad & Breitengrad & Höhe & 1 & 2 & 3 & 4 & 5 & 6 & 7 & 8 & 9 & 10 & 11 & 12 & $\Sigma$ & $\overline{\mathrm{x}}$ & $\sigma$ \\
\hline NT4 & $39^{\circ} 31^{\prime} 22,6^{\prime \prime} \mathrm{N}$ & $20^{\circ} 45^{\prime} 54,8^{\prime \prime} \mathrm{E}$ & $1621 \mathrm{~m}$ & - & - & - & - & 1 & - & 1 & - & - & 1 & - & - & 3 & $57,58 \%$ & 27,0 \\
\hline NT6 & $39^{\circ} 31^{\prime} 13,5 " \mathrm{~N}$ & $20^{\circ} 46^{\prime} 03,0^{\prime \prime} \mathrm{E}$ & $1720 \mathrm{~m}$ & 5 & 1 & - & 1 & - & - & - & 2 & 1 & 1 & - & 1 & 12 & $35,23 \%$ & 39,4 \\
\hline NT5 & $39^{\circ} 31^{\prime} 12,1^{\prime \prime} \mathrm{N}$ & $20^{\circ} 45^{\prime} 57,4 " \mathrm{E}$ & $1667 \mathrm{~m}$ & 6 & 2 & 5 & - & - & - & - & - & - & - & - & - & 13 & $7,06 \%$ & 7,5 \\
\hline NTD & $39^{\circ} 31^{\prime} 11,7^{\prime \prime} \mathrm{N}$ & $20^{\circ} 46^{\prime} 12,5^{\prime \prime} \mathrm{E}$ & $1757 \mathrm{~m}$ & 3 & - & - & - & 1 & - & - & - & - & 1 & 6 & 4 & 15 & $73,28 \%$ & 40,9 \\
\hline NT7 & $39^{\circ} 31^{\prime} 11,5^{\prime \prime} \mathrm{N}$ & $20^{\circ} 46^{\prime} 09,4^{\prime \prime} \mathrm{E}$ & $1769 \mathrm{~m}$ & 4 & 1 & 2 & - & - & - & 1 & - & 1 & - & - & - & 9 & $18,16 \%$ & 28,1 \\
\hline NT8 & $39^{\circ} 31^{\prime} 09,6 " \mathrm{~N}$ & $20^{\circ} 46^{\prime} 14,3^{\prime \prime} \mathrm{E}$ & $1747 \mathrm{~m}$ & 3 & 1 & - & 1 & - & 1 & - & - & - & - & 2 & - & 8 & $31,83 \%$ & 42,5 \\
\hline NT9 & $39^{\circ} 31^{\prime} 03,6^{\prime \prime} \mathrm{N}$ & $20^{\circ} 46^{\prime} 20,4 " \mathrm{E}$ & $1775 \mathrm{~m}$ & 5 & 2 & 1 & - & - & - & - & - & - & - & - & - & 8 & $2,73 \%$ & 5,6 \\
\hline NTG & $39^{\circ} 31^{\prime} 02,8^{\prime \prime} \mathrm{N}$ & $20^{\circ} 46^{\prime} 28,7^{\prime \prime} \mathrm{E}$ & $1765 \mathrm{~m}$ & 8 & 1 & 1 & 2 & - & - & - & - & - & - & 5 & 2 & 19 & $39,48 \%$ & 46,1 \\
\hline NT11 & $39^{\circ} 30^{\prime} 57,6^{\prime \prime} \mathrm{N}$ & $20^{\circ} 46^{\prime} 20,0^{\prime \prime} \mathrm{E}$ & $1776 \mathrm{~m}$ & 5 & 1 & 1 & - & - & - & 1 & - & - & - & - & - & 8 & $10,46 \%$ & 20,4 \\
\hline NT16 & $39^{\circ} 30^{\prime} 57,2^{\prime \prime} \mathrm{N}$ & $20^{\circ} 46^{\prime} 27,0^{\prime \prime} \mathrm{E}$ & $1759 \mathrm{~m}$ & 8 & - & - & - & - & - & - & 1 & 2 & 1 & 1 & - & 13 & $30,91 \%$ & 41,4 \\
\hline NT15 & $39^{\circ} 30^{\prime} 56,6^{\prime \prime} \mathrm{N}$ & $20^{\circ} 46^{\prime} 22,8^{\prime \prime} \mathrm{E}$ & $1759 \mathrm{~m}$ & 6 & 2 & 2 & - & - & - & 1 & - & - & - & - & - & 11 & $8,24 \%$ & 16,1 \\
\hline NT12 & $39^{\circ} 30^{\prime} 54,3^{\prime \prime} \mathrm{N}$ & $20^{\circ} 46^{\prime} 16,3^{\prime \prime} \mathrm{E}$ & $1761 \mathrm{~m}$ & 1 & - & - & - & - & - & - & 1 & 2 & 1 & 2 & 1 & 8 & $74,16 \%$ & 32,5 \\
\hline NT13 & $39^{\circ} 30^{\prime} 52,7^{\prime \prime} \mathrm{N}$ & $20^{\circ} 46^{\prime} 12,4 " \mathrm{E}$ & $1738 \mathrm{~m}$ & 5 & 2 & 1 & - & - & - & - & - & - & - & - & - & 8 & $2,96 \%$ & 6,2 \\
\hline NT14 & $39^{\circ} 30^{\prime} 52,2^{\prime \prime} \mathrm{N}$ & $20^{\circ} 46^{\prime} 05,2^{\prime \prime} \mathrm{E}$ & $1684 \mathrm{~m}$ & 5 & 1 & - & 1 & - & 1 & - & - & - & - & - & - & 8 & $9,67 \%$ & 17,6 \\
\hline NTS3 & $39^{\circ} 30^{\prime} 39,6^{\prime \prime} \mathrm{N}$ & $20^{\circ} 45^{\prime} 48,0^{\prime \prime} \mathrm{E}$ & $1650 \mathrm{~m}$ & 2 & - & - & - & - & - & - & - & - & - & - & - & 2 & $0,00 \%$ & 0,0 \\
\hline NTS4 & $39^{\circ} 30^{\prime} 38,9^{\prime \prime} \mathrm{N}$ & $20^{\circ} 45^{\prime} 57,3^{\prime \prime} \mathrm{E}$ & $1689 \mathrm{~m}$ & 7 & - & 1 & - & - & - & - & - & - & - & - & - & 8 & $1,42 \%$ & 4,0 \\
\hline NTS2 & $39^{\circ} 30^{\prime} 36,4^{\prime \prime} \mathrm{N}$ & $20^{\circ} 45^{\prime} 35,0^{\prime \prime} \mathrm{E}$ & $1603 \mathrm{~m}$ & 3 & - & - & - & - & - & - & - & - & - & 1 & - & 4 & $22,84 \%$ & 45,7 \\
\hline NTS1 & $39^{\circ} 30^{\prime} 30,7^{\prime \prime} \mathrm{N}$ & $20^{\circ} 45^{\prime} 31,3^{\prime \prime} \mathrm{E}$ & $1540 \mathrm{~m}$ & 2 & 1 & 1 & - & - & 1 & - & - & - & - & - & - & 5 & $12,35 \%$ & 18,8 \\
\hline
\end{tabular}

\subsubsection{Merkmalsverteilung auf dem Tomaros-Südmassiv}

Allgemeine Übersicht: Das Untersuchungsgebiet des Südtomaros umfasst 43 Fundorte, von denen insgesamt 579 Individuen stammen. Wie auch schon in den vorangegangenen Betrachtungsweisen werden zunächst allen diesen Individuen Hybridindices zugeteilt (Abb. 66). Es ergibt sich, dass 205 (35,4\%) der Individuen dieses Gebietes der St. clavatusReferenzgruppe entsprechen (Hybridindex 1). Dem Index 2 werden 50 (8,6\%) der Tiere, 
Index 3 - 46 (7,9\%) und Hybridindex 4 - 21 (5,2\%) der Individuen zugeteilt. Zwischen 24 $(4,1 \%)$ und $18(3,1 \%)$ Individuen werden den Hybridindices $5-8$ zugeordnet. $\mathrm{Zu}$ den Hybridindices die Individuen beschreiben, die der St. rubicundus-Referenzgruppe ähneln (Index 9 bis 11) werden 43 (7,4\%) Tiere dem Index 9, 45 (7,8\%) Individuen dem Index 10 und 34 (5,9\%) Individuen werden dem Index 11 zugeordnet. Insgesamt 42 (7,3\%) Individuen entsprechen der St. rubicundus-Referenzgruppe (Index 12).

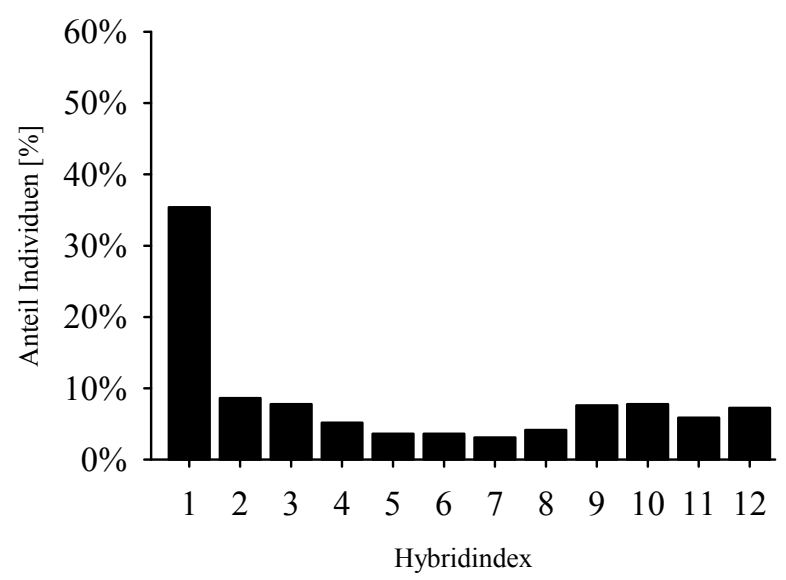

Abb. 66 Gesamtverteilung der Hybridindices (Antennenmerkmal) auf dem Süd-Tomaros

Zusammenfassend lässt sich feststellen, dass in diesem Untersuchungsbiet Individuen, die auf Grundlage der Antennenmorphologie der St. clavatus-Referenzgruppe entsprechen (Index1), die größte Gruppe bilden. Am wenigsten sind Tiere mit intermediären Merkmalen vertreten, wobei aber Individuen jedes Hybridindexes vertreten sind. Auch Tiere, die der St. rubicundus-Referenzgruppe voll entsprechen, sind nicht sehr stark vertreten.

Es werden nun wiederum die durchschnittlichen Merkmalsanteile jedes Fundortes in Form eines Kreisdiagramms sowie die Verteilung der Hybridindices in Abb. 67 und Abb. 68 graphisch dargestellt und im Folgenden beschrieben. 


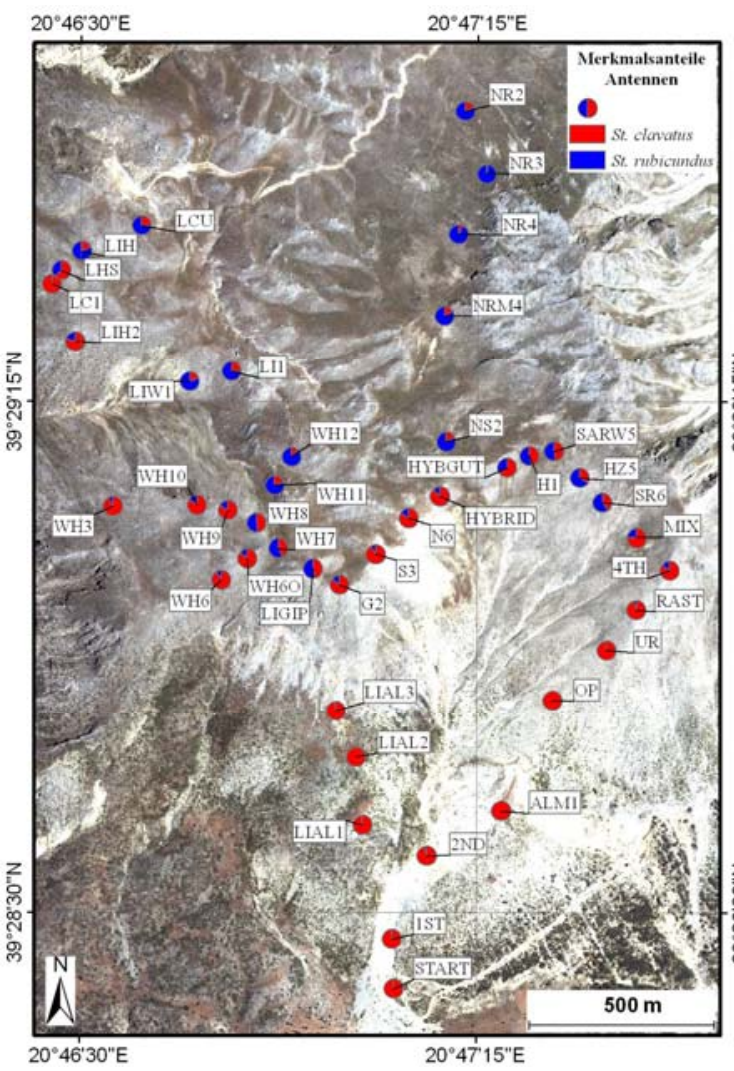

Abb. 67 Verteilung der durchschnittlichen Merkmalsanteile [\%] auf dem Südtomaros

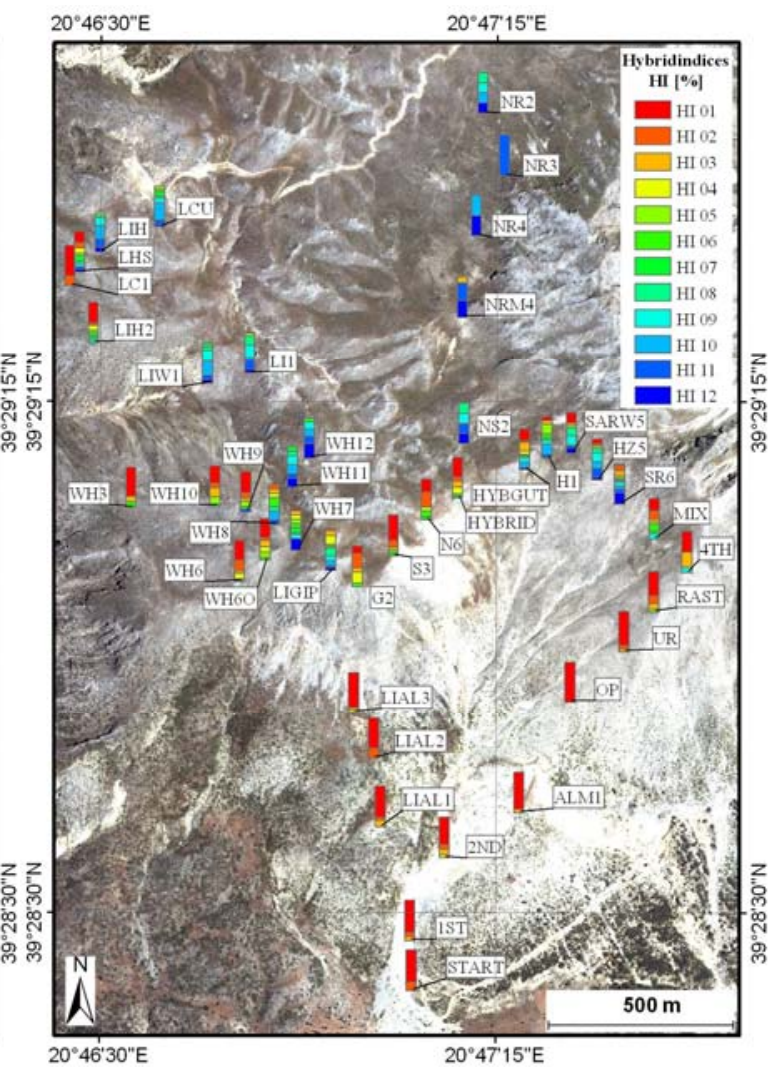

Abb. 68 Anteil der Individuen mit entsprechenden Hybridindices für jeden Fundort auf dem Südtomaros

Merkmalsverteilung im Detail: Beginnend von Norden sind vier Fundorte zu finden (Tab. 21), die durchschnittliche Merkmalsanteile zwischen 83,09\% (NR2) und 98,05 \% (NR3) aufweisen, also den St. rubicundus der Referenzgruppe ähneln. Die Individuen dieser Fundorte besitzen Hybridindices zwischen 8 und 12, wobei die meisten Tiere sich Indices zwischen 10 und 12 zuordnen lassen.

Tab. 21 Merkmalsverteilung der Fundorte (GPS) im nördlichen Teilgebiet des Südtomaros

\begin{tabular}{|c|c|c|c|c|c|c|c|c|c|c|c|c|c|c|c|c|c|c|}
\hline \multicolumn{4}{|c|}{ Geographische Parameter } & \multicolumn{13}{|c|}{ Hybridindices } & \multicolumn{2}{|c|}{ Merkmalsanteil } \\
\hline GPS & Längengrad & Breitengrad & Höhe & 1 & 2 & 3 & 4 & 5 & 6 & 7 & 8 & 9 & 10 & 11 & 12 & $\Sigma$ & $\overline{\mathrm{x}}$ & $\sigma$ \\
\hline NR2 & $39^{\circ} 29^{\prime} 40,6^{\prime \prime} \mathrm{N}$ & $20^{\circ} 47^{\prime} 13,6^{\prime \prime} \mathrm{E}$ & $1636 \mathrm{~m}$ & - & - & - & - & - & - & - & 1 & 1 & 1 & - & 1 & 4 & $83,09 \%$ & 15,5 \\
\hline NR3 & $39^{\circ} 29^{\prime} 35,0^{\prime \prime} \mathrm{N}$ & $20^{\circ} 47^{\prime} 16,0^{\prime \prime} \mathrm{E}$ & $1717 \mathrm{~m}$ & - & - & - & - & - & - & - & - & - & - & 2 & - & 2 & $98,05 \%$ & 2,2 \\
\hline NR4 & $39^{\circ} 29^{\prime} 29,8^{\prime \prime} \mathrm{N}$ & $20^{\circ} 47^{\prime} 12,9^{\prime \prime} \mathrm{E}$ & $1761 \mathrm{~m}$ & - & - & - & - & - & - & - & - & - & 2 & - & 2 & 4 & $92,72 \%$ & 8,5 \\
\hline NRM4 & $39^{\circ} 29^{\prime} 22,6^{\prime \prime} \mathrm{N}$ & $20^{\circ} 47^{\prime} 11,3 " \mathrm{E}$ & $1811 \mathrm{~m}$ & - & - & - & - & - & - & - & - & 1 & - & 3 & 3 & 7 & $93,30 \%$ & 8,7 \\
\hline
\end{tabular}

Östlich von diesem Gebiet, in dem Tiere mit hauptsächlich St. rubicundus Merkmalen gefunden worden sind, gibt es ein Teilgebiet mit heterogener Merkmalsstruktur. Individuen mit einer Antennenmorphologie, die einer der beiden Referenzgruppen entspricht, aber auch Individuen mit intermediären Merkmalen sind hier zu finden (Tab. 22). Von den sieben Fundorten dieses Teilgebietes liegt der Merkmalsanteil der östlich liegenden Fundorte zwischen 75,29\% (LCU) und 82,02\% (LiH). Die dort gefangenen Individuen lassen sich in 
Hybridindices zwischen 7 und 12 einteilen. Vereinzelt sind aber auch Individuen mit Hybridindices von 4 bis 6 zu finden. Östlich dieser sieben Fundorte befinden sich weitere drei Fundorte die durchschnittliche Merkmalsanteile zwischen 35,9\% (LHS) und 1,39\% (LC1) haben. Am Fundort LHS findet man sowohl intermediäre Individuen als auch Tiere beider Referenzgruppen, wobei mehr Individuen der Art St. clavatus zugeordnet werden können als St. rubicundus. Individuen der beiden östlichen Fundorte dieses Teilgebietes lassen sich den Hybridindices 1 bis 9 zuordnen. Diese große Varianz ist aber ausschließlich bei den Tieren des Fundortes LiH2 zu beobachten, die Individuen des Fundortes LC1 werden den Hybridindices 1 und 2 zugeordnet. Auffällig ist hier, dass Individuen mit einem Hybridindex von 1 stark vertreten sind.

Tab. 22 Merkmalsverteilung der Fundorte (GPS) im westlichen Teilgebiet des Südtomaros

\begin{tabular}{|c|c|c|c|c|c|c|c|c|c|c|c|c|c|c|c|c|c|c|}
\hline \multicolumn{4}{|c|}{ Geographische Parameter } & \multicolumn{13}{|c|}{ Hybridindices } & \multicolumn{2}{|c|}{ Merkmalsanteil } \\
\hline GPS & Längengrad & Breitengrad & Höhe & 1 & 2 & 3 & 4 & 5 & 6 & 7 & 8 & 9 & 10 & 11 & 12 & $\Sigma$ & $\overline{\mathrm{x}}$ & $\sigma$ \\
\hline LCU & $39^{\circ} 29^{\prime} 30,5^{\prime \prime} \mathrm{N}$ & $20^{\circ} 46 ' 36,9^{\prime \prime} \mathrm{E}$ & $1545 \mathrm{~m}$ & - & - & - & 1 & - & 1 & 2 & 1 & 2 & 7 & 2 & 1 & 17 & $75,29 \%$ & 21,1 \\
\hline $\mathbf{L i H}$ & $39^{\circ} 29^{\prime} 28,3^{\prime \prime} \mathrm{N}$ & $20^{\circ} 46 ' 30,1^{\prime \prime E}$ & $1543 m$ & - & - & - & 1 & - & - & - & 1 & 3 & 5 & 3 & 2 & 15 & $82,02 \%$ & 18,0 \\
\hline LI1 & $39^{\circ} 29^{\prime} 17,7^{\prime \prime} \mathrm{N}$ & $20^{\circ} 46^{\prime} 47,2^{\prime \prime} \mathrm{E}$ & $1661 \mathrm{~m}$ & - & - & - & - & 1 & - & - & 3 & 4 & 1 & 3 & 1 & 13 & $75,96 \%$ & 17,6 \\
\hline LiW1 & $39^{\circ} 29^{\prime} 16,7^{\prime \prime} \mathrm{N}$ & $20^{\circ} 46^{\prime} 42,4^{\prime \prime} \mathrm{E}$ & $1621 \mathrm{~m}$ & - & - & - & - & - & - & 1 & 1 & 3 & 4 & 1 & 1 & 11 & $80,46 \%$ & 13,4 \\
\hline LHS & $39^{\circ} 29^{\prime} 26,6^{\prime \prime} \mathrm{N}$ & $20^{\circ} 46^{\prime} 27,8^{\prime \prime} \mathrm{E}$ & $1536 \mathrm{~m}$ & 4 & 2 & - & 2 & 1 & - & 2 & 1 & 1 & - & 1 & 1 & 15 & $35,90 \%$ & 35,3 \\
\hline LC1 & $39^{\circ} 29^{\prime} 25,4^{\prime \prime} \mathrm{N}$ & $20^{\circ} 46 \prime 26,7^{\prime \prime} \mathrm{E}$ & $1536 \mathrm{~m}$ & 6 & 2 & - & - & - & - & - & - & - & - & - & - & 8 & $1,39 \%$ & 3,4 \\
\hline LiH2 & $39^{\circ} 29^{\prime} 20,3^{\prime \prime} \mathrm{N}$ & $20^{\circ} 46 \prime 29,4^{\prime \prime} \mathrm{E}$ & $1581 \mathrm{~m}$ & 8 & 1 & - & 2 & 1 & 1 & 1 & 1 & 1 & - & - & - & 16 & $20,54 \%$ & 27,0 \\
\hline
\end{tabular}

Auf dem Südhang des Tomaros-Südmassives sind Individuen zu finden, die sich den Hybridindices von 1 bis 3 zuordnen lassen (Tab. 23). Somit entsprechen diese der St. clavatusReferenzgruppe oder ähneln dieser stark. Man findet aber auch vereinzelt Tiere $(\mathrm{N}=3)$, die den Hybridindices 4 und 5 zugeteilt werden können. Zusammenfassung: Der durchschnittliche Merkmalsanteil der Individuen der 10 Fundorte in diesem Teilgebiet liegt bei 2,16\% und variiert zwischen $0 \%(\mathrm{OP})$ und 5,24 \% (Rast). Individuen mit einem Hybridindex von 1 sind auch hier mit Abstand am stärksten vertreten.

Tab. 23 Merkmalsverteilung der Fundorte (GPS) im östlichen Teilgebiet des Südtomaros

\begin{tabular}{|c|c|c|c|c|c|c|c|c|c|c|c|c|c|c|c|c|c|c|}
\hline \multicolumn{4}{|c|}{ Geographische Parameter } & \multicolumn{13}{|c|}{ Hybridindices } & \multicolumn{2}{|c|}{ Merkmalsantei } \\
\hline GPS & Längengrad & Breitengrad & Höhe & 1 & 2 & 3 & 4 & 5 & 6 & 7 & 8 & 9 & 10 & 11 & 12 & $\Sigma$ & $\bar{x}$ & $\sigma$ \\
\hline Rast & $39^{\circ} 28^{\prime} 56,7^{\prime \prime} \mathrm{N}$ & $20^{\circ} 47^{\prime} 33,1^{\prime \prime} \mathrm{E}$ & $1812 \mathrm{~m}$ & 9 & 3 & 2 & - & 1 & - & - & - & - & - & - & - & 15 & $5,24 \%$ & 10,0 \\
\hline UR & $39^{\circ} 28^{\prime} 53,2^{\prime \prime} \mathrm{N}$ & $20^{\circ} 47^{\prime} 29,7^{\prime \prime E}$ & $1753 \mathrm{~m}$ & 10 & 1 & 1 & - & - & - & - & - & - & - & - & - & 12 & $1,63 \%$ & 3,9 \\
\hline OP & $39^{\circ} 28^{\prime} 48,7^{\prime \prime} \mathrm{N}$ & $20^{\circ} 47^{\prime} 23,6^{\prime \prime} \mathrm{E}$ & $1644 m$ & 11 & - & - & - & - & - & - & - & - & - & - & - & 11 & $\mathbf{0 , 0 0 \%}$ & 0,0 \\
\hline LiAl3 & $39^{\circ} 28^{\prime} 47,8^{\prime \prime} \mathrm{N}$ & $20^{\circ} 46^{\prime} 59,0^{\prime \prime} \mathrm{E}$ & $1611 \mathrm{~m}$ & 13 & - & 1 & 1 & - & - & - & - & - & - & - & - & 15 & $2,41 \%$ & 6,6 \\
\hline LiAl2 & $39^{\circ} 28^{\prime} 43,7 " \mathrm{~N}$ & $20^{\circ} 47^{\prime} 01,3 " E$ & $1543 \mathrm{~m}$ & 6 & 2 & - & - & - & - & - & - & - & - & - & - & 8 & $0,59 \%$ & 1,1 \\
\hline ALM1 & $39^{\circ} 28^{\prime} 38,9^{\prime \prime} \mathrm{N}$ & $20^{\circ} 47^{\prime} 17,8$ "E & $1514 m$ & 11 & - & 1 & - & - & - & - & - & - & - & - & - & 12 & $0,98 \%$ & 3,4 \\
\hline LiAl1 & $39^{\circ} 28^{\prime} 37,7^{\prime \prime} \mathrm{N}$ & $20^{\circ} 47^{\prime} 02,1^{\prime \prime} \mathrm{E}$ & $1476 \mathrm{~m}$ & 10 & 1 & 2 & - & - & - & - & - & - & - & - & - & 13 & $2,76 \%$ & 5,5 \\
\hline 2nd & $39^{\circ} 28^{\prime} 35,0^{\prime \prime} \mathrm{N}$ & $20^{\circ} 47^{\prime} 09,4^{\prime \prime} \mathrm{E}$ & $1425 \mathrm{~m}$ & 10 & 2 & 2 & 1 & - & - & - & - & - & - & - & - & 15 & $4,65 \%$ & 7,7 \\
\hline 1st & $39^{\circ} 28^{\prime} 27,7 " \mathrm{~N}$ & $20^{\circ} 47^{\prime} 05,5^{\prime \prime} \mathrm{E}$ & $1368 \mathrm{~m}$ & 8 & 1 & 1 & - & - & - & - & - & - & - & - & - & 10 & $1,85 \%$ & 4,3 \\
\hline Start & $39^{\circ} 28^{\prime} 23,3^{\prime \prime} \mathrm{N}$ & $20^{\circ} 47^{\prime} 05,6 " \mathrm{E}$ & $1326 \mathrm{~m}$ & 8 & 2 & - & - & - & - & - & - & - & - & - & - & 10 & $1,48 \%$ & 3,2 \\
\hline
\end{tabular}


Zwischen dem Nord- und Südhang des Tomaros-Südmassives findet man ein langgestrecktes, schmales Gebiet, in dem die Tiere eine durchaus heterogene Merkmalsstruktur besitzen. Es erstreckt sich vom Westhang über den westlichen kleineren Gipfel und über den Sattel des Südtomaros bis unterhalb des Hauptgipfels. An der nördlichen Kante dieses Teilgebietes befinden sich drei Fundorte, an denen die Tiere Merkmalsanteile zwischen $81,42 \%$ und 88,24\% besitzen (Tab. 24). Die meisten Individuen dieser Fundorte besitzen Hybridindices zwischen 9 und 12, vereinzelt aber auch Indices von 6 bis 8. Diese Individuen zeigen somit zum größten Teil bezüglich des Antennenmerkmals eine starke Ähnlichkeit mit der Art St. rubicundus. Es sind aber auch vereinzelt Individuen mit intermediären Merkmalen (Index 6 bis 8) zu finden. Südlich dieser drei Fundorte erstrecken sich in der Ost-West-Ausdehnung acht Fundorte (GPS-Punkte), an denen sowohl intermediäre Individuen als auch Individuen, die eine der beiden Referenzarten entsprechen, $\mathrm{zu}$ finden sind. Somit wurden in diesem Teilgebiet Individuen aller Hybridindices gefunden (Tab. 24). Der durchschnittliche Merkmalsanteil liegt bei 53,5\% und variiert zwischen 33,57\% (Hybgut) und 66,70\% (HZ5). Am südlichen und westlichen Rand dieses langgestreckten Teilgebietes wurden Tiere gefangen, die hauptsächlich den Hybridindices von 1 bis 7 zuzuordnen sind, und somit St. clavatus- bis intermediäre Antennenmerkmale aufweisen. Der mittlere Merkmalsanteil der dreizehn Fundorte liegt bei 13,77 \% variiert aber zwischen 8,5\% (S3) und $24,8 \%$ (MIX).

Tab. 24 Merkmalsverteilung der Fundorte (GPS) im Bereich des Sattels des Südtomaros

\begin{tabular}{|c|c|c|c|c|c|c|c|c|c|c|c|c|c|c|c|c|c|c|}
\hline \multicolumn{4}{|c|}{ Geographische Parameter } & \multicolumn{13}{|c|}{ Hybridindices } & \multicolumn{2}{|c|}{ Merkmalsanteil } \\
\hline GPS & Längengrad & Breitengrad & Höhe & 1 & 2 & 3 & 4 & 5 & 6 & 7 & 8 & 9 & 10 & 11 & 12 & $\boldsymbol{\Sigma}$ & $\bar{x}$ & $\sigma$ \\
\hline NS2 & $39^{\circ} 29^{\prime} 11,4 " \mathrm{~N}$ & $20^{\circ} 47^{\prime} 11,5^{\prime \prime} \mathrm{E}$ & $1778 \mathrm{~m}$ & - & - & - & - & - & - & - & 1 & 1 & - & 1 & 1 & 4 & $83,21 \%$ & 15,4 \\
\hline WH12 & $39^{\circ} 29^{\prime} 10,1^{\prime \prime} \mathrm{N}$ & $20^{\circ} 466^{\prime} 53,9 " \mathrm{E}$ & $1751 \mathrm{~m}$ & - & - & - & - & - & 1 & - & - & 2 & 2 & 2 & 4 & 11 & $88,24 \%$ & 16,0 \\
\hline WH11 & $39^{\circ} 29^{\prime} 07,6^{\prime \prime} \mathrm{N}$ & $20^{\circ} 46^{\prime} 52,0^{\prime \prime} \mathrm{E}$ & $1741 \mathrm{~m}$ & - & - & - & - & - & - & 1 & 1 & 2 & 2 & 1 & 2 & 9 & $81,42 \%$ & 15,0 \\
\hline SARW5 & $39^{\circ} 29^{\prime} 10,6^{\prime \prime} \mathrm{N}$ & $20^{\circ} 47^{\prime} 23,7^{\prime \prime} \mathrm{E}$ & $1884 \mathrm{~m}$ & 4 & - & 1 & - & 1 & - & - & 3 & 4 & - & 1 & 2 & 16 & $52,59 \%$ & 37,9 \\
\hline H1 & $39^{\circ} 29^{\prime} 10,2^{\prime \prime} \mathrm{N}$ & $20^{\circ} 47^{\prime} 20,9 " \mathrm{E}$ & $1854 \mathrm{~m}$ & 1 & - & 1 & - & 3 & 1 & 1 & - & 1 & 3 & 1 & - & 12 & $53,55 \%$ & 31,1 \\
\hline Hybgut & $39^{\circ} 29^{\prime} 09,2^{\prime \prime} \mathrm{N}$ & $20^{\circ} 47^{\prime} 18,4^{\prime \prime} \mathrm{E}$ & $1833 m$ & 7 & 1 & 5 & 2 & - & 1 & 1 & - & 3 & 3 & - & 1 & 24 & $33,57 \%$ & 34,8 \\
\hline HZ5 & $39^{\circ} 29^{\prime} 08,3^{\prime \prime} \mathrm{N}$ & $20^{\circ} 47^{\prime} 26,6^{\prime \prime} \mathrm{E}$ & $1888 \mathrm{~m}$ & 2 & 1 & - & - & 1 & - & - & 2 & 4 & 3 & 4 & 1 & 18 & $66,70 \%$ & 33,9 \\
\hline SR6 & $39^{\circ} 29^{\prime} 06,1^{\prime \prime} \mathrm{N}$ & $20^{\circ} 47^{\prime} 29,2^{\prime \prime} \mathrm{E}$ & $1885 \mathrm{~m}$ & 1 & 3 & 3 & 1 & 1 & 1 & 1 & - & 2 & 2 & 2 & 7 & 24 & $60,09 \%$ & 39,6 \\
\hline LiGip & $39^{\circ} 29^{\prime} 00,3^{\prime \prime} \mathrm{N}$ & $20^{\circ} 46^{\prime} 56,4^{\prime \prime} \mathrm{E}$ & $1803 m$ & - & 1 & 3 & 5 & 1 & 1 & - & 5 & 3 & 3 & 1 & 2 & 25 & $53,87 \%$ & 31,3 \\
\hline WH8 & $39^{\circ} 29^{\prime} 04,3^{\prime \prime} \mathrm{N}$ & $20^{\circ} 466^{\prime} 50,0^{\prime \prime} \mathrm{E}$ & $1761 \mathrm{~m}$ & - & 1 & 1 & 1 & - & 2 & - & 1 & - & 2 & 1 & - & 9 & $51,74 \%$ & 33,7 \\
\hline WH7 & $39^{\circ} 29^{\prime} 02,1$ "N & $20^{\circ} 46^{\prime} 52,5^{\prime \prime} \mathrm{E}$ & $1784 m$ & 1 & - & 2 & 2 & 2 & 2 & 2 & 1 & 2 & - & - & 5 & 19 & $56,29 \%$ & 33,6 \\
\hline N6 & $39^{\circ} 29^{\prime} 04,7^{\prime \prime} \mathrm{N}$ & $20^{\circ} 47^{\prime} 07,3$ "E & $1793 m$ & 4 & 5 & - & 1 & - & 2 & 1 & - & - & - & - & - & 13 & $15,46 \%$ & 20,6 \\
\hline Hybrid & $39^{\circ} 29^{\prime} 06,6^{\prime \prime} \mathrm{N}$ & $20^{\circ} 47^{\prime} 10,8^{\prime \prime} \mathrm{E}$ & $1790 \mathrm{~m}$ & 9 & 2 & 3 & 3 & - & 1 & 1 & - & - & - & - & - & 19 & $11,95 \%$ & 17,0 \\
\hline MIX & $39^{\circ} 29^{\prime} 03,0^{\prime \prime} \mathrm{N}$ & $20^{\circ} 47^{\prime} 33,1^{\prime \prime} \mathrm{E}$ & $1880 \mathrm{~m}$ & 4 & 3 & 1 & - & 1 & 2 & 1 & - & 2 & - & - & - & 14 & $24,86 \%$ & 27,8 \\
\hline S3 & $39^{\circ} 29^{\prime} 01,6^{\prime \prime} \mathrm{N}$ & $20^{\circ} 47^{\prime} 03,5^{\prime \prime} \mathrm{E}$ & $1792 \mathrm{~m}$ & 9 & 2 & 1 & - & - & 1 & 1 & - & - & - & - & - & 14 & $8,58 \%$ & 17,7 \\
\hline 4th & $39^{\circ} 29^{\prime} 00,2^{\prime \prime} \mathrm{N}$ & $20^{\circ} 47^{\prime} 36,8^{\prime \prime} \mathrm{E}$ & $1876 \mathrm{~m}$ & 4 & - & 3 & - & - & - & - & - & 1 & - & - & - & 8 & $14,41 \%$ & 24,3 \\
\hline WH10 & $39^{\circ} 29^{\prime} 05,8^{\prime \prime} \mathrm{N}$ & $20^{\circ} 46^{\prime} 43,2^{\prime \prime} \mathrm{E}$ & $1710 \mathrm{~m}$ & 7 & 2 & 4 & - & 3 & - & - & 1 & - & - & - & - & 17 & $14,71 \%$ & 18,3 \\
\hline WH9 & $39^{\circ} 29^{\prime} 05,4^{\prime \prime} \mathrm{N}$ & $20^{\circ} 46^{\prime} 46,7^{\prime \prime} \mathrm{E}$ & $1736 \mathrm{~m}$ & 8 & 2 & 1 & - & 2 & 1 & - & - & - & - & 1 & - & 15 & $15,71 \%$ & 26,1 \\
\hline WH3 & $39^{\circ} 29^{\prime} 05,7^{\prime \prime} \mathrm{N}$ & $20^{\circ} 466^{\prime} 33,7^{\prime \prime} \mathrm{E}$ & $1617 \mathrm{~m}$ & 5 & - & 1 & - & - & 1 & - & - & - & - & - & - & 7 & $8,51 \%$ & 16,5 \\
\hline WH6O & $39^{\circ} 29^{\prime} 01,2^{\prime \prime} \mathrm{N}$ & $20^{\circ} 46^{\prime} 48,9^{\prime \prime} \mathrm{E}$ & $1768 \mathrm{~m}$ & 7 & 1 & 2 & 2 & 2 & 1 & - & - & - & - & - & - & 15 & $13,71 \%$ & 15,7 \\
\hline WH6 & $39^{\circ} 28^{\prime} 59,3^{\prime \prime} \mathrm{N}$ & $20^{\circ} 46^{\prime} 46,0^{\prime \prime} \mathrm{E}$ & $1750 \mathrm{~m}$ & 7 & 4 & 1 & 2 & - & - & 1 & - & - & - & - & - & 15 & $8,96 \%$ & 16,2 \\
\hline G2 & $39^{\circ} 28^{\prime} 58,9^{\prime \prime} \mathrm{N}$ & $20^{\circ} 46^{\prime} 59,4^{\prime \prime} \mathrm{E}$ & $1795 m$ & 2 & 4 & 1 & 3 & - & 1 & - & - & - & - & - & - & 11 & $14,65 \%$ & 14,8 \\
\hline
\end{tabular}


Zusammenfassung: Auf dem Südmassiv des Südtomaros findet man auf dem Nordhang Tiere, die der St. rubicundus-Referenzgruppe entsprechen oder ihr doch ähneln. Hingegen sind auf dem Südhang größtenteils Heuschrecken gefunden worden, die der St. clavatusReferenzgruppe gleichen. Zwischen diesen beiden Gebieten erstreckt sich eine Zone, in der Individuen mit Antennen gefunden worden sind, die eine der beiden Arten zugeordnet werden können. Es sind aber auch hier eine nicht unbeträchtliche Anzahl von Heuschrecken mit intermediären Antennenmerkmalen gefunden worden.

\subsubsection{Merkmalsverteilung auf dem Osthang des Südtomaros}

Für die Untersuchung der Merkmalsverteilung auf den Osthang des Tomaros-Südmassives sind die Antennen von 172 Individuen von 19 Fundorten vermessen worden. Es konnten somit dem Hybridindex 1 siebzehn (9,6\%) Individuen zugeordnet werden (Abb. 69). Keines der hier gefangenen Individuen konnte den Hybridindex 2 zugeordnet werden. Den Hybridindices 3 bis 9 wurden jeweils zwischen 4 (2,3\%) und 8 (4,5) Individuen zugeordnet. Die meisten Individuen dieses Gebietes konnten den Hybridindices 10 bis 12 zugeteilt werden. Demnach erhielten 25 (14,1\%) Individuen Index 10, 49 (27,7\%) Tiere wurden Index 11 und 47 (26,6\%) Individuen dem Hybridindex 12 zugeteilt. Auf Grundlage dessen sind in diesem Gebiet hauptsächlich Individuen zu finden, die entweder der St. rubicundusReferenzgruppe ähneln (Index 10 und 11) oder dieser voll entsprechen und demnach einen Hybridindex von 12 erhalten.

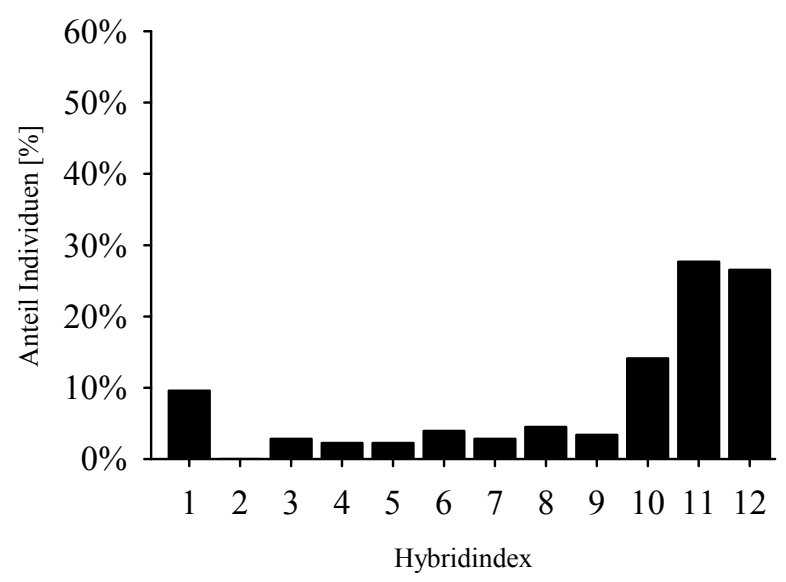

Abb. 69 Gesamtverteilung der Hybridindices (Antennenmerkmal) auf dem Osthang des Süd-Tomaros 


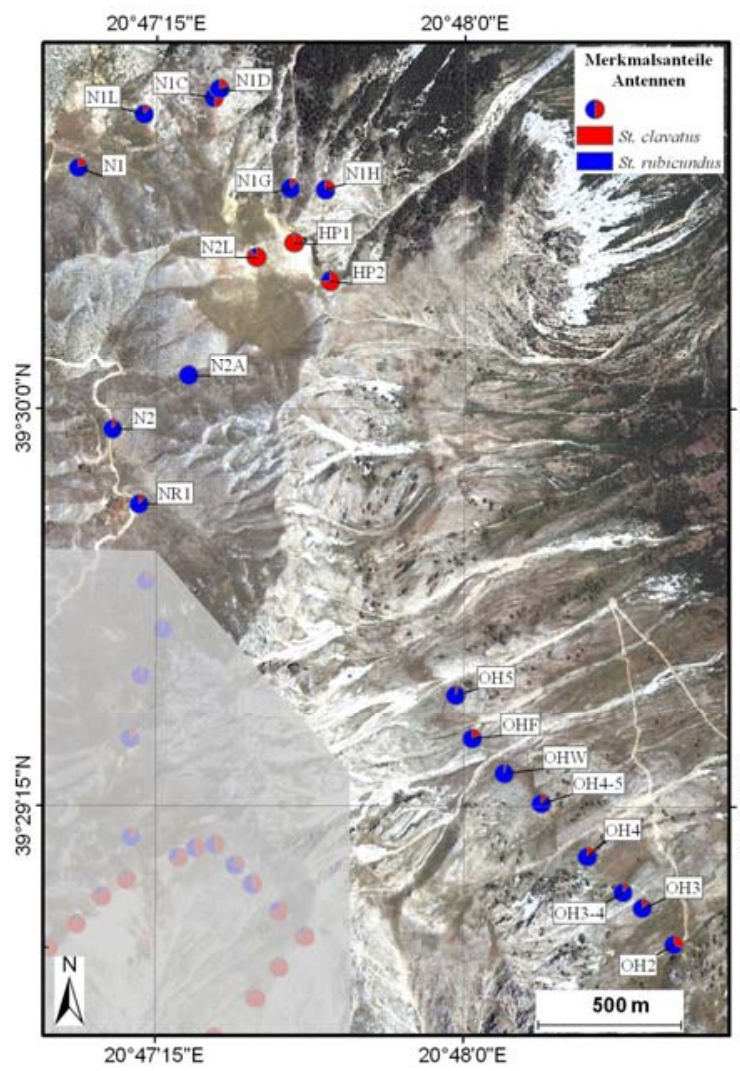

Abb. 70 Verteilung der durchschnittlichen Merkmalsanteile [\%] auf dem Osthang des Südtomaros

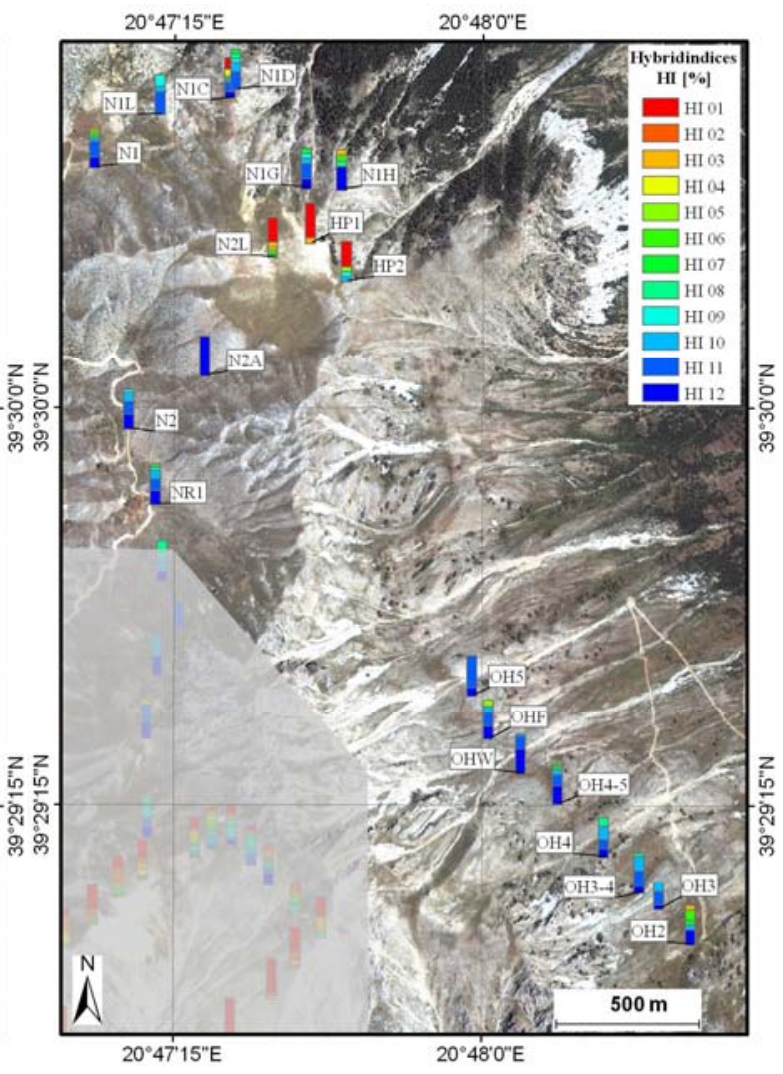

Abb. 71 Anteil der Individuen mit entsprechenden Hybridindices für jeden Fundort auf dem Osthang des Südtomaros

Zur Detailbetrachtung wird der Osthang des Südtomaros in ein nördliches und ein südliches Teilgebiet untergliedert (Abb. 70 und Abb. 71). Im nördlichen Teil liegen 12 Fundorte, an denen die Tiere sehr unterschiedliche Merkmalscharaktere aufweisen. An sieben der zwölf Fundorte sind hauptsächlich Individuen gefunden worden, deren Antennen den Hybridindices von 5 bis 12 - in der Mehrzahl 10 - 12 - zuzuteilen sind (Tab. 25). Die Merkmalsanteile der Individuen dieser sieben Fundorte liegen durchschnittlich bei 85,3\%, variieren aber zwischen 91,73\% beim GPS-Punkt N2 und 74,82\% bei N1H. Zwischen diesen Fundorten mit einem Merkmalscharakter, die der St. rubicundus-Referenzgruppe sehr ähnelt, sind auch an einigen Fundorten Individuen mit Antennenmerkmalen im Bereich von St. clavatus (zwei Fundorte) gesammelt worden. An diesen zwei GPS-Punkten sind Individuen mit Hybridindices zwischen 1 und 7 gefunden worden. Diese Tiere besitzen mehr Merkmale die der Art St. clavatus mehr ähneln als der Art St. rubicundus. Dies drückt sich in Merkmalsanteilen von 11,96\% (N2L) und 1,66 \% (HP1) aus. Bei einem der Fundorte sind Individuen mit Hybridindices, die den Referenzgruppen entsprechen, aber auch inter- 
mediäre Individuen gefunden worden. Der durchschnittliche Merkmalsanteil der Individuen dieses Fundortes (N1C) liegt bei 51,45\%.

Tab. 25 Merkmalsverteilung der Fundorte (GPS) im nördlichen Teilgebiet des Osthanges des Südtomaros

\begin{tabular}{|c|c|c|c|c|c|c|c|c|c|c|c|c|c|c|c|c|c|c|}
\hline \multicolumn{4}{|c|}{ Geographische Parameter } & \multicolumn{13}{|c|}{ Hybridindices } & \multicolumn{2}{|c|}{ Merkmalsanteil } \\
\hline GPS & Längengrad & Breitengrad & Höhe & 1 & 2 & 3 & 4 & 5 & 6 & 7 & 8 & 9 & 10 & 11 & 12 & $\Sigma$ & $\overline{\mathrm{x}}$ & $\sigma$ \\
\hline N1D & $39^{\circ} 30^{\prime} 36,2^{\prime \prime} \mathrm{N}$ & $20^{\circ} 47^{\prime} 24,1 " \mathrm{E}$ & $1378 \mathrm{~m}$ & - & & - & - & . & 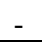 & 1 & 1 & 1 & 2 & 4 & - & 9 & $1,80 \%$ & 15,6 \\
\hline N1L & $39^{\circ} 30^{\prime} 33,3^{\prime \prime} \mathrm{N}$ & $20^{\circ} 47^{\prime} 13,1 " \mathrm{E}$ & $1392 \mathrm{~m}$ & - & - & - & - & - & - & - & - & 2 & 1 & 4 & - & 7 & $8,79 \%$ & 10,4 \\
\hline N1C & $39^{\circ} 30^{\prime} 35,2^{\prime \prime} \mathrm{N}$ & $20^{\circ} 47^{\prime} 23,3^{\prime \prime E}$ & $1398 \mathrm{~m}$ & 5 & 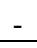 & - & 3 & - & 1 & - & 1 & - & 1 & 4 & 3 & 18 & $45 \%$ & 42,5 \\
\hline N1 & $39^{\circ} 30^{\prime} 27,3^{\prime \prime N}$ & $20^{\circ} 47^{\prime} 03,6^{\prime \prime E}$ & $1438 m$ & - & - & - & - & 1 & 1 & - & 1 & - & 1 & 6 & 4 & 15 & $84,27 \%$ & 22,7 \\
\hline N1G & $39^{\circ} 30^{\prime} 25,0^{\prime \prime} \mathrm{N}$ & $20^{\circ} 47^{\prime} 34,6^{\prime \prime E}$ & $1489 m$ & - & - & - & - & - & - & 1 & - & 1 & 1 & 3 & 2 & 8 & $87,92 \%$ & 16,7 \\
\hline $\mathrm{N} 1 \mathrm{H}$ & $39^{\circ} 30^{\prime} 24,9^{\prime \prime} \mathrm{N}$ & $20^{\circ} 47^{\prime} 39,7 " \mathrm{E}$ & $1468 m$ & - & - & 1 & - & - & 1 & - & 1 & - & - & - & 4 & 7 & $74,82 \%$ & 34,2 \\
\hline HP1 & $39^{\circ} 30^{\prime} 18,9^{\prime \prime} \mathrm{N}$ & $20^{\circ} 47^{\prime} 35,1{ }^{\prime \prime E}$ & $1591 \mathrm{~m}$ & 6 & - & 1 & - & - & - & - & - & - & - & - & - & 7 &, $66 \%$ & 4,4 \\
\hline N2L & $39^{\circ} 30^{\prime} 17,3^{\prime \prime} \mathrm{N}$ & $20^{\circ} 47^{\prime} 29,6 " \mathrm{E}$ & $1637 \mathrm{~m}$ & 9 & - & 2 & 1 & 1 & 1 & 1 & - & - & - & - & - & 15 & $11,96 \%$ & 18,0 \\
\hline HP2 & $39^{\circ} 30^{\prime} 14,5^{\prime \prime} \mathrm{N}$ & $20^{\circ} 47^{\prime} 40,4^{\prime \prime E}$ & $1401 \mathrm{~m}$ & 5 & - & - & - & 1 & - & - & - & 1 & 1 & - & - & 8 & $23,76 \%$ & 36,3 \\
\hline N2 & $39^{\circ} 29^{\prime} 57,6^{\prime \prime} \mathrm{N}$ & $20^{\circ} 47^{\prime} 08,6 " \mathrm{E}$ & $1524 \mathrm{~m}$ & - & - & - & - & - & - & - & - & 1 & 3 & 4 & 5 & 13 & $91,73 \%$ & 8,5 \\
\hline NR1 & $39^{\circ} 29^{\prime} 49,1^{\prime \prime} \mathrm{N}$ & $20^{\circ} 47^{\prime} 12,5^{\prime \prime E}$ & $1578 m$ & - & - & - & - & - & - & 1 & - & 1 & 3 & 4 & 5 & 14 & $90,52 \%$ & 13,3 \\
\hline
\end{tabular}

Im südlichen Teil des Osthanges (Tab. 26) liegen weitere acht Fundorte von Tieren mit einem durchschnittlichen Merkmalsanteil von 87,8\%. Zwischen den einzelnen Fundorten können die Merkmalsanteile der Individuen aber durchaus zwischen 97,38\% am Fundort (OHW) und 69,24 \% am Fundort OH2 variieren. Die hier vorkommenden Individuen wurden hauptsächlich den Hybridindices von 10 bis 12 zugeordnet. Nichtsdestotrotz existieren hier aber auch einige Individuen, die auf Basis ihrer Antennenmorphologie in Hybridindices von 5 bis 8 oder sogar dem Index 3 zugeordnet werden können. Demnach wurden in diesem Teilgebiet Individuen gefunden, deren Antennen der Art St. rubicundus sehr ähneln (Index 10 und 11) oder dieser Art entsprechen (Index 12). Zusätzlich sind aber auch einzelne Individuen mit intermediären Antennenmerkmalen (Index 5 bis 8) zu finden.

Tab. 26 Merkmalsverteilung der Fundorte (GPS) im südlichen Teilgebiet des Osthanges des Südtomaros

\begin{tabular}{|c|c|c|c|c|c|c|c|c|c|c|c|c|c|c|c|c|c|c|}
\hline \multicolumn{4}{|c|}{ Geographische Parameter } & \multicolumn{13}{|c|}{ Hybridindices } & \multicolumn{2}{|c|}{ Merkmalsanteil } \\
\hline GPS & Längengrad & Breitengrad & Höhe & 1 & 2 & 3 & 4 & 5 & 6 & 7 & 8 & 9 & 10 & 11 & 12 & $\Sigma$ & $\overline{\mathrm{x}}$ & $\sigma$ \\
\hline OH5 & $39^{\circ} 29^{\prime} 27,6^{\prime \prime} \mathrm{N}$ & $20^{\circ} 47^{\prime} 58,8^{\prime \prime} \mathrm{E}$ & $1425 \mathrm{~m}$ & - & - & - & - & - & - & - & - & - & - & 4 & 1 & 5 & $95,73 \%$ & 4,0 \\
\hline OHF & $39^{\circ} 29^{\prime} 22,6^{\prime \prime} \mathrm{N}$ & $20^{\circ} 48^{\prime} 01,2^{\prime \prime E}$ & $1420 \mathrm{~m}$ & - & - & - & - & 1 & - & - & - & - & 1 & 2 & 2 & 6 & $84,25 \%$ & 27,2 \\
\hline OHW & $39^{\circ} 29^{\prime} 18,7^{\prime \prime} \mathrm{N}$ & $20^{\circ} 48^{\prime} 05,9^{\prime \prime E}$ & $1405 \mathrm{~m}$ & - & - & - & - & - & - & - & - & - & 1 & 5 & 10 & 16 & $7,38 \%$ & 4,9 \\
\hline $\mathrm{OH} 4-5$ & $39^{\circ} 29^{\prime} 15,3^{\prime \prime} \mathrm{N}$ & $20^{\circ} 48^{\prime} 11,3^{\prime \prime E}$ & $1377 \mathrm{~m}$ & - & - & - & - & - & 1 & 1 & - & - & 2 & 4 & 7 & 15 & $90,60 \%$ & 17,1 \\
\hline $\mathrm{OH} 4$ & $39^{\circ} 29^{\prime} 09,2^{\prime \prime} \mathrm{N}$ & $20^{\circ} 48^{\prime} 18,1 " \mathrm{E}$ & $1358 \mathrm{~m}$ & - & - & - & - & - & - & - & 2 & - & 3 & 2 & 2 & 9 & $86,68 \%$ & 12,2 \\
\hline $\mathrm{OH} 3-4$ & $39^{\circ} 29^{\prime} 05,2^{\prime \prime} \mathrm{N}$ & $20^{\circ} 48^{\prime} 23,3^{\prime \prime E}$ & $1338 \mathrm{~m}$ & - & - & - & - & - & - & - & 1 & - & 4 & 4 & 2 & 11 & $89,79 \%$ & 10,6 \\
\hline $\mathrm{OH} 3$ & $39^{\circ} 29^{\prime} 03,4^{\prime \prime} \mathrm{N}$ & $20^{\circ} 48^{\prime} 26,2^{\prime \prime} \mathrm{E}$ & $1321 \mathrm{~m}$ & - & - & - & - & - & - & - & 1 & - & 4 & 3 & 1 & 9 & $88,71 \%$ & 12,2 \\
\hline $\mathrm{OH} 2$ & $39^{\circ} 28^{\prime} 59,3^{\prime \prime} \mathrm{N}$ & $20^{\circ} 48^{\prime} 30,8^{\prime \prime E}$ & $1294 m$ & - & - & 1 & - & - & 2 & 1 & - & - & 1 & - & 3 & 8 & $69,24 \%$ & 30,9 \\
\hline
\end{tabular}

Zusammenfassend lässt sich sagen, dass im Gebiet des Osthanges im Norden Fundorte mit heterogener Merkmalsstruktur und in südlichen Teil Fundorte liegen, an denen hauptsäch- 
lich Individuen mit Merkmalen im Bereich der St. rubicundus-Referenzgruppe vorkommen.

\subsubsection{Bestimmung der Merkmalskline auf Grundlage der Antennenmorphologie}

Mit Hilfe der durchschnittlichen Merkmalsanteile an den einzelnen Fundorten kann nun die geographische Position der Kontaktzonen zwischen St. clavatus und St. rubicundus auf dem Tomaros bestimmt werden. Die durchschnittlichen Merkmalsanteile der Individuen jedes Fundortes (GPS-Punktes) bilden die Datengrundlage für die distanzbasierte Interpolation in ArcGIS des Antennenmerkmals. Nachdem die Mittelpunkte der Hybridzone bestimmt wurden, wird die Merkmalsverteilung durch Interpolation bestimmt.

\subsubsection{Interpolierte Merkmalsverteilung des Antennenmerkmals auf dem Tomaros}

Auf einem Raster, das durch distanzbasierte Interpolation des Antennenmerkmals auf dem Tomaros entstanden ist, werden, wie auch bei der Interpolation des Flügelmerkmals, drei Kontaktzonen deutlich (Abb. 72) . Die erste der drei Kontaktzonen (Kontaktzone I) befindet sich auf dem Südtomaros und erstreckt sich vom nordwestlichen Teil des Südtomaros über den Sattel bis hin zum südlichen Teil des Osthangs (Abb. 72 rechts). Eine sehr kleine Kontaktzone ist im nördlichen Teil des Südtomaros (Kontaktzone II) und eine weitere im südlichen Teil des Nordtomaros (Kontaktzone III) zu finden. Des Weiteren wird durch Interpolation ein zusammenhängendes Bild der Merkmalsverteilung des Antennenmerkmals erkennbar.

Vom nördlichen Teil des Nordtomaros erstreckt sich über ein Drittel des Bergrückens ein Gebiet, an dem der interpolierte Merkmalsanteil der Individuen durchschnittlich im Bereich der Art St. clavatus liegt (Merkmalsanteil 0\%-2,5\%). Weiter südlich schließt sich ein Gebiet an, an dem der durchschnittliche Merkmalsanteil der Individuen zwischen 2,5\% und $10 \%$ liegt. Im südlichen Teil des Nordtomaros sind Gebiete zu finden, in denen die den Individuen intermediäre Merkmale aufweisen. Zwischen diesen Zonen erstrecken sich aber größere Gebiete, in denen Individuen mit Merkmalsanteilen zu finden sind, die entweder der Art St. clavatus entsprechen oder dieser ähneln (Abb. 72). 

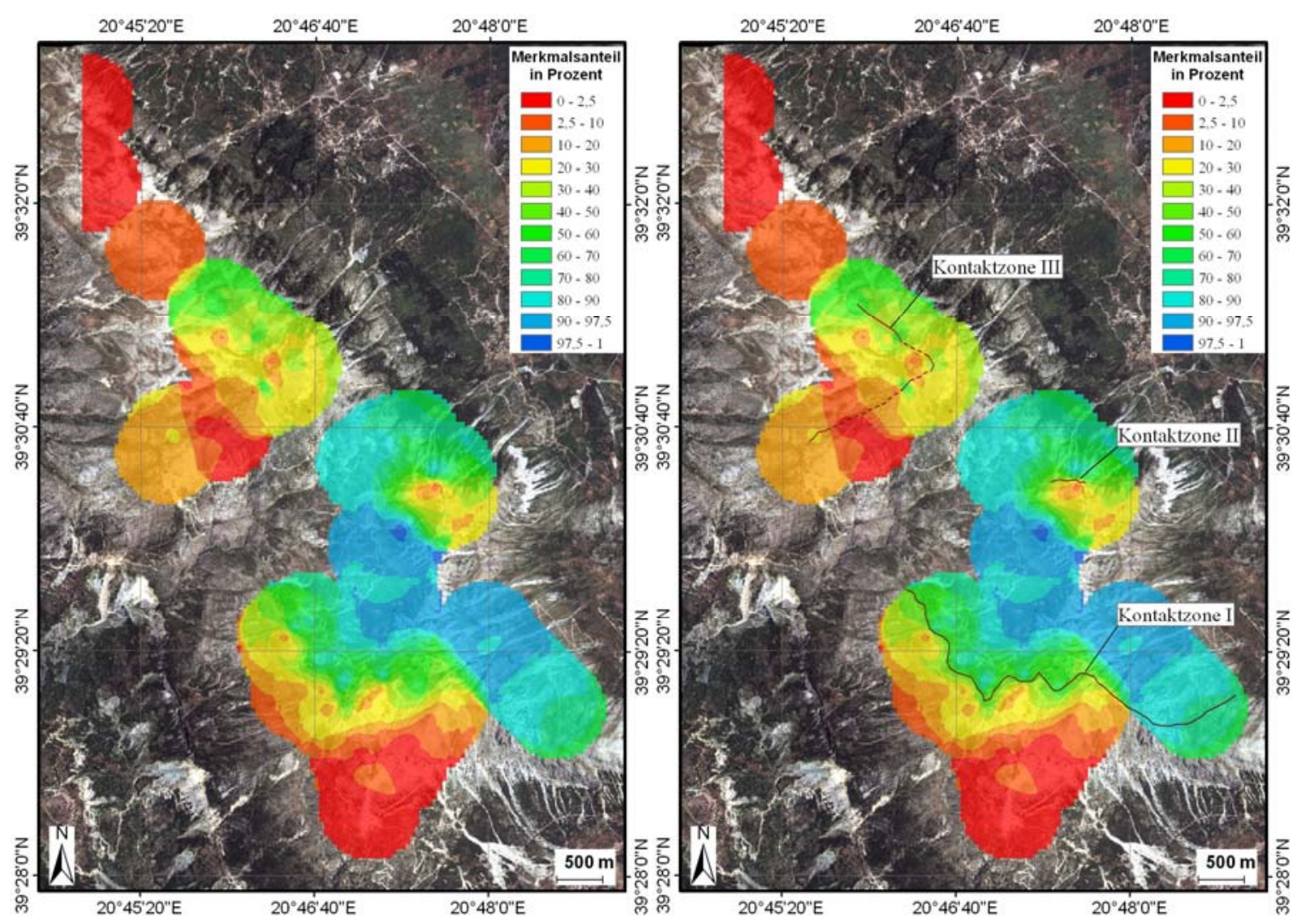

Abb. 72 Rasterstruktur der interpolierten Merkmalsverteilung (rechts) und interpolierte Merkmalsverteilung nach Glättung (bilineare Interpolation) links.

Ganz im Nordosten des Südtomaros findet man, wie auch bei der Interpolation der Flügelmerkmale, ein kleines Gebiet, in dem die Individuen interpolierte Merkmalsanteile im intermediären Bereich (Kontaktzone II) zeigen. Außerdem kommen an vereinzelten Fundorten Individuen vor, die der Art Stenobothrus clavatus sehr ähnlich sind. Umgeben sind diese Gebiete von interpolierten Rasterflächen, die auf Individuen hinweisen, deren Antennemerkmale der Art. St. rubicundus ähneln oder dieser entsprechen.

Wie bereits beschrieben, erstreckt sich eine langgezogene Kontaktzone (Kontaktzone I) quer über den Südtomaros (Abb. 72). Nördlich dieser Kontaktzone sind Gebiete zu finden, in denen die Merkmalsanteile durchschnittlich zwischen $80 \%$ und 100\% liegen. Es gibt also kein Gebiet, in dem interpolierte Merkmalsanteile der Individuen ermittelt worden sind, die der St. rubicundus-Referenzgruppe entsprechen. Auf der südlichen Seite sind hauptsächlich Gebiete zu finden, in denen die Individuen interpolierte durchschnittliche Merkmalsanteile von $0 \%$ bis $2,5 \%$ aufweisen. Somit entsprechen diese Individuen auf dem Antennenmerkmal der Art St. clavatus. 


\subsubsection{Merkmalskline der Antennen}

Im Folgenden wird nun die Verteilung des Antennenmerkmals im Bezug zu den Kontaktzonen betrachtet. Fundorte, die sich auf der nördlichen sowie der östlichen Seite der Kontaktzonen befinden, erhalten bei der Entfernungsbestimmung durch ArcGIS einen negativen und die Fundorte der südlichen und westlichen Seiten einen positiven Entfernungswert. Somit können die Merkmalskline des Antennenmerkmals ausgehend von den drei Kontaktzonen im Folgenden beschrieben werden. Im Zuge dessen werden jeder Kontaktzone die Fundorte zugeordnet die zu der jeweiligen Kontaktzone den geringsten Abstand aufweisen.

\section{Kontaktzone I}

Die durchschnittlichen Merkmalsanteile der Individuen der 51 Fundorte, die zur Kontaktzone I den kürzesten Abstand aufweisen, beschreiben in der Graphik (Abb. 73) eine sigmoide Kurve.

Der Bereich, in dem Fundorte mit durchschnittlichen intermediären Merkmalen (Merkmalsanteil 40\%-60\%) zu finden sind, ist $160 \mathrm{~m}$ breit. Ausgehend von dieser Zone erhöhen sich die durchschnittlichen Merkmalsanteile der Individuen an den Fundorten in nördlicher Richtung bis zu einem Abstand von 253 m kontinuierlich und pendeln sich von dort an zwischen durchschnittlich $84 \%$ und $99 \%$ ein.

In südlicher Richtung hingegen verringert sich der durchschnittliche Merkmalsanteil an den einzelnen Fundorten mit zunehmender Entfernung von $36 \%$ bis auf 6,5\%. Ab einem Abstand von 337 m zur Kontaktzone I pendelt der durchschnittliche Merkmalsanteil der Individuen der einzelnen Fundorte konstant zwischen 6\% und 0\%. 


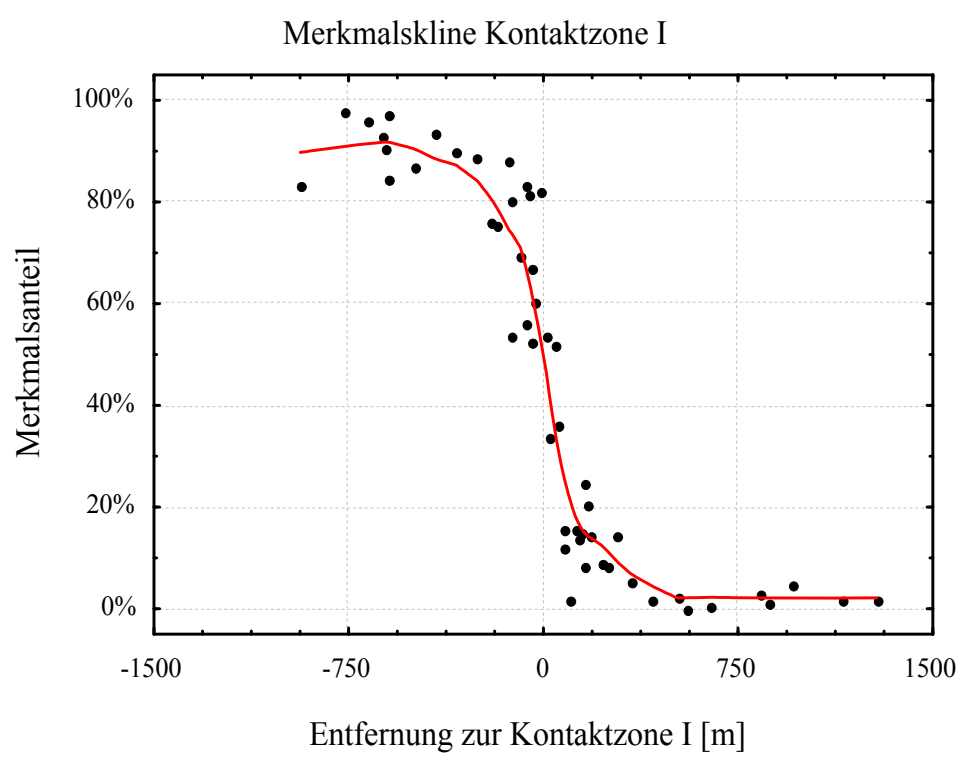

Abb. 73 Antennen-Merkmalskline im Bereich der Kontaktzone I. Lowess-Glättung $(-)$ h=0,25

\section{Kontaktzone II}

Der Verlauf der Kline im Bereich der Kontaktzone II (Abb. 74) zeigt ein anderes Bild als in Kontaktzone I. Hier findet man lediglich einen Fundort, an dem die Individuen einen durchschnittlichen Merkmalsanteil im intermediären Bereich, also zwischen $40 \%$ und $60 \%$, aufweisen.

Auf der südlichen Seite der Kontaktzone II sind in einem Abstand von $660 \mathrm{~m}$ und $820 \mathrm{~m}$ zwei Fundorte, an denen der durchschnittliche Merkmalsanteil zwischen 91\% und 93\% liegt. Zwischen $37 \mathrm{~m}$ und $190 \mathrm{~m}$ in nördlicher Richtung von der ermittelten Kontaktzone entfernt befinden sich drei Fundorte, an denen die Individuen durchschnittliche Merkmalsanteile zwischen 30\% und 0\% aufweisen. Somit sind diese Individuen auf Grundlage des Antennenmerkmals der Art St. clavatus sehr ähnlich oder entsprechen dieser. Weiter in nördlicher Richtung sind im Abstand von $352 \mathrm{~m}$ und $378 \mathrm{~m}$ zwei weitere Fundorte gelegen, an denen Heuschrecken mit durchschnittlichen Merkmalsanteilen von $75 \%$ bis $88 \%$ zu finden sind. Der einzige Fundort, an dem ein durchschnittlicher Merkmalsanteil im intermediären Bereich ermittelt worden ist, befindet sich in einem Abstand von ca. $200 \mathrm{~m}$ zur Kontaktzone II. Drei Fundorte, an denen die Individuen durchschnittliche Merkmalsanteile zwischen $82 \%$ und 89\% aufweisen, sind in nördlicher Richtung in einem Abstand von 748 $\mathrm{m}$ bis $771 \mathrm{~m}$ zu Kontaktzone zu finden. Diese Individuen ähneln den Individuen der Art St. rubicundus. 


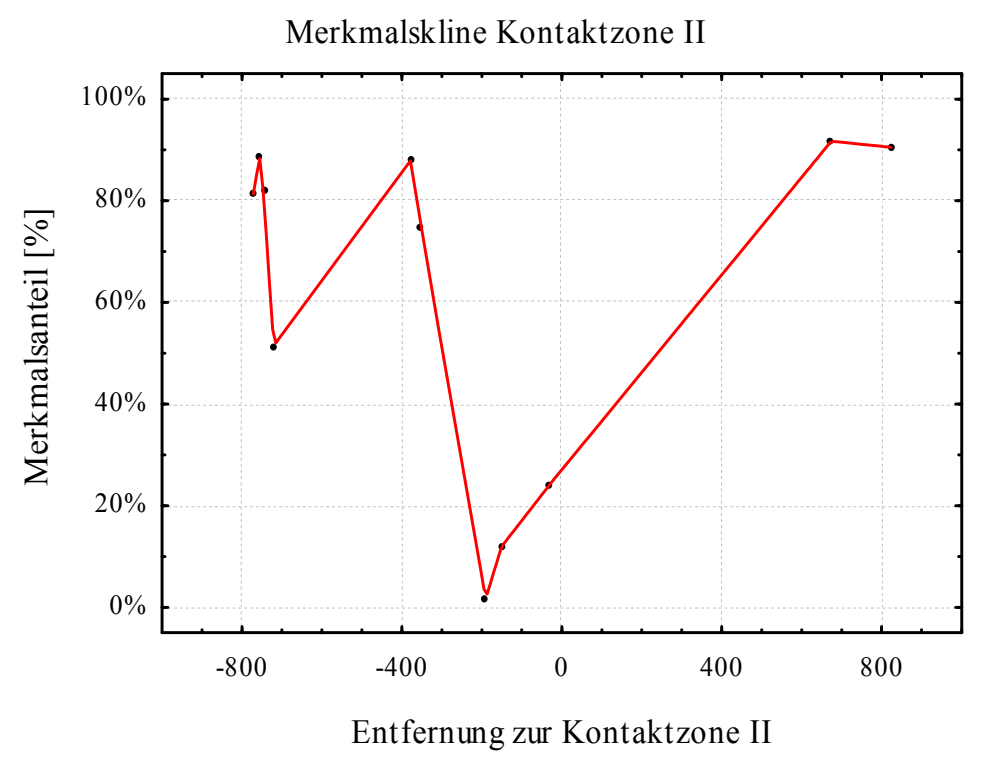

Abb. 74 Antennen-Merkmalskline im Bereich der Kontaktzone II. Lowess-Glättung $(-)$ h=0,25

\section{Kontaktzone III}

Bei der Betrachtung der Kline III des Antennenmerkmals auf dem Tomaros fällt auf, dass sich südlich der Kontaktzone legendlich 5 der 21 Fundorte befinden (Abb. 75). Die Kurve fällt ausgehend von der Kontaktzone zuerst steil in Richtung Norden ab und pegelt sich dann um einen niedrigen Merkmalsanteilswert ein. In einem Abstand von $41 \mathrm{~m}$ und $49 \mathrm{~m}$ befinden sich zwei Fundorte, an denen die Individuen durchschnittliche Merkmalsanteile von $73 \%$ oder $74 \%$ aufweisen. Die dort zu findenden Individuen haben bezüglich des Antennenmerkmals Ähnlichkeiten mit St. rubicundus. Zwei weitere Fundorte, an denen die Merkmalsanteile durchschnittlich zwischen $40 \%$ und $60 \%$ betragen, findet man in einem Abstand von $51 \mathrm{~m}$ bzw. $53 \mathrm{~m}$ zur Kontaktzone III. Nördlich der Kontaktzone III ist der Großteil der Fundorte zu finden. Bis zu einer Entfernung von 206 m fällt der durchschnittliche Merkmalsanteil der Individuen der einzelnen Fundorte kontinuierlich von 35\% bis auf $2 \%$ ab. Von diesem Punkt an verändert sich der durchschnittliche Merkmalsanteil nur leicht und sinkt im Bereich der beiden letzten Fundorte, die einen Abstand von 1948 m bzw. 2664 m zur Hybridzone aufweisen, bis auf durchschnittliche Merkmalsanteile von 1\% bzw. 0\% ab. Diese Tiere entsprechen somit der St. clavatus-Referenzgruppe. 


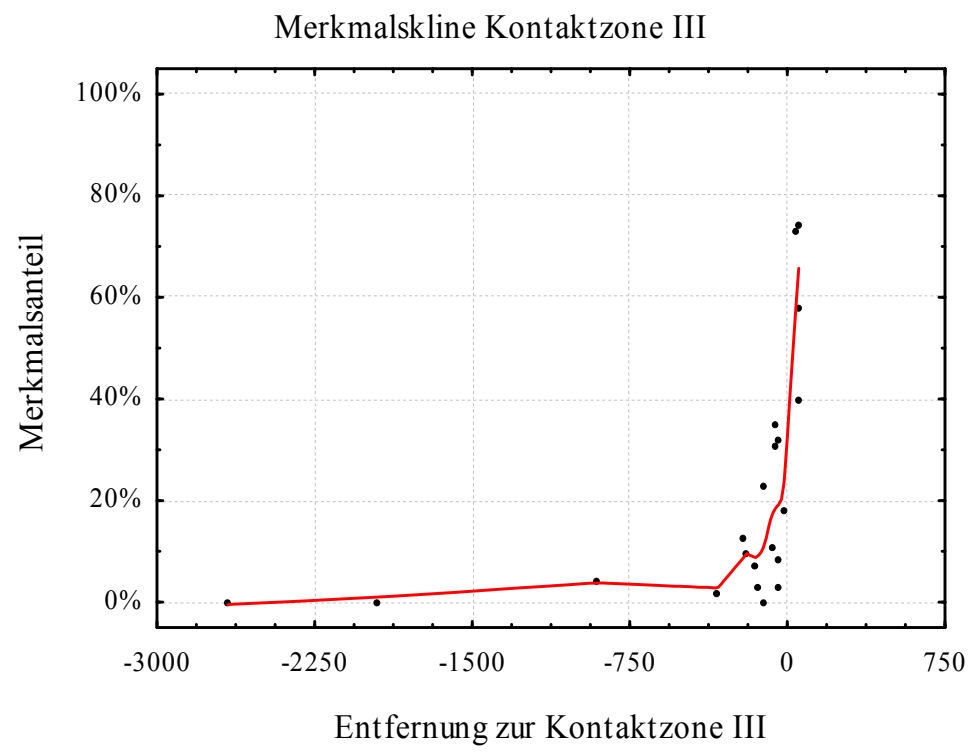

Abb. 75 Antennen-Merkmalskline im Bereich der Kontaktzone III. Lowess-Glättung $(-)$ h=0,25

\section{Zusammenfassung}

Trotz der unterschiedlichen Gesamtstruktur der Merkmalskline der Antennen gibt es gerade zwischen Kontaktzone I, II und III viele Gemeinsamkeiten. Der Bereich, in dem die durchschnittlichen Merkmalsanteile zwischen 40\% und 60\% liegen, ist zwischen $50 \mathrm{~m}$ und $200 \mathrm{~m}$ breit. Ausgehend vom Zentrum der Kontaktzone ist eine Zone zu finden, in der sich der durchschnittliche Merkmalsanteil der Fundorte mit zunehmender Entfernung rasch ändert. Ab einem Abstand von ca. $350 \mathrm{~m}$ zu Kontaktzone regelt sich der Merkmalsanteil meist auf einen konstanten Wert ein. Eine Ausnahme bilden die Merkmalsanteile der Individuen der einzelnen Fundorte auf der nördlichen Seite der Kontaktzone I, die ab ca. $250 \mathrm{~m}$ von Fundort zu Fundort sehr variabel sind.

\subsection{Vergleich zwischen Flügel- und Antennenmorphologie}

Ob es einen Zusammenhang zwischen Antennen- und Flügelmorphologie gibt, soll im Folgenden genauer untersucht werden. Hierzu wurden von 988 Individuen (631 § und 357ㅇ), von denen jeweils der Vorderflügel und die Antenne vermessen wurden, untersucht.

Der Vergleich der Flügel- und Antennenmerkmale bei den Männchen zeigt eine starke Korrelation von $\mathrm{r}=0,94$. Vergleicht man aber die Merkmalsanteile der Individuen des Tomaros miteinander, verringert sich die Korrelation leicht und liegt bei $\mathrm{r}=0,932$. 
Korrelationsmatrize der Merkmalsanteile

Korrelation: $r=, 94201$

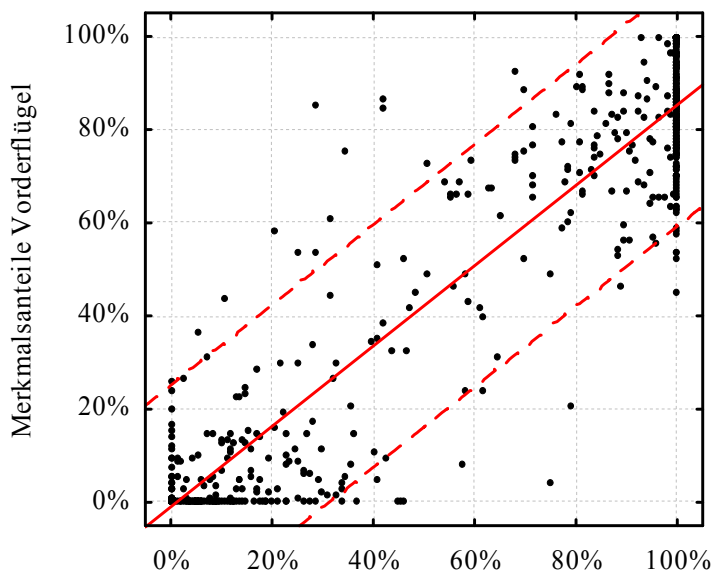

0,95 Prog.Int. Merkmalsanteile Vorderflügel
Korrelationsmatrize der Merkmalsanteile Tomaros

Korrelation: $r=, 93237$

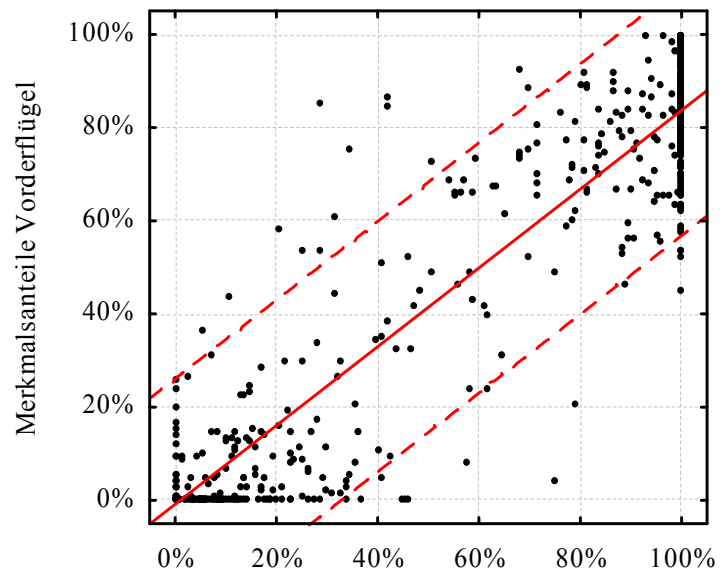

0,95 Prog.Int. Merkmalsanteile Antennen

Abb. 76 Korrelationsanalyse der Merkmalsanteile der Vorderflügel- und Antennenmerkmale bei allen Männchen und Männchen des Tomaros. Regressionsgerade (-) und Regressionsbänder (- -) (Prognostiziertes Intervall: Niveau 0,95 )

Bei der Untersuchung der Merkmale der Weibchen wurde im Vergleich zu den Männchen eine sehr viel schwächere Korrelation errechnet, nämlich nur $r=0,811$. Klammert man nun die Individuen der allopatrischen Populationen aus und untersucht ausschließlich die Individuen des Tomaros, vermindert sich diese weiter auf $r=0,78$.

Abschließend lässt sich aber sagen, dass Flügel- und Antennenmerkmale der Individuen miteinander korrelieren. Die Korrelation ist aber bei den Männchen weitaus stärker als bei den Weibchen. Werden bei der Berechnung die Individuen der allopatrischen Populationen ausgeklammert, verringert sich die Korrelation kaum. 
Korrelationsmatrize der Merkmalsanteile Korrelation: $r=, 81172$

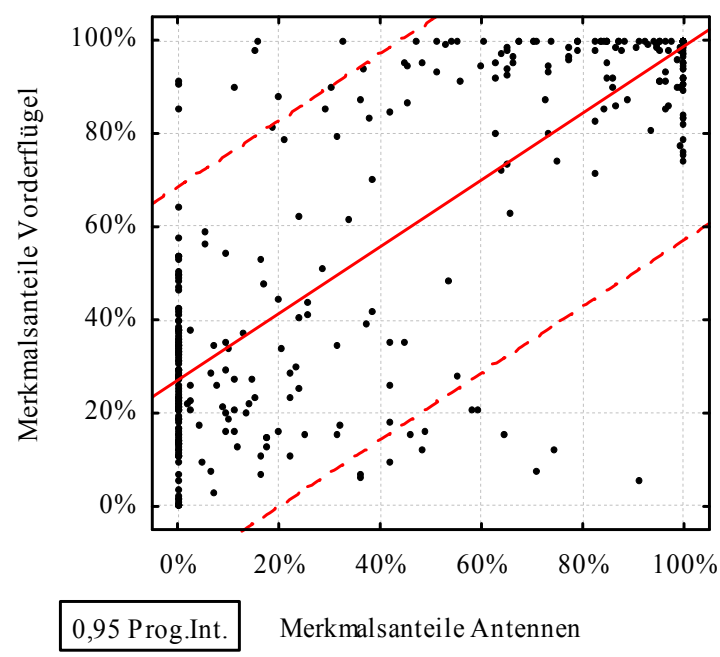

Korrelationsmatrize der Merkmalsanteile Tomaros Korrelation: $r=, 78198$

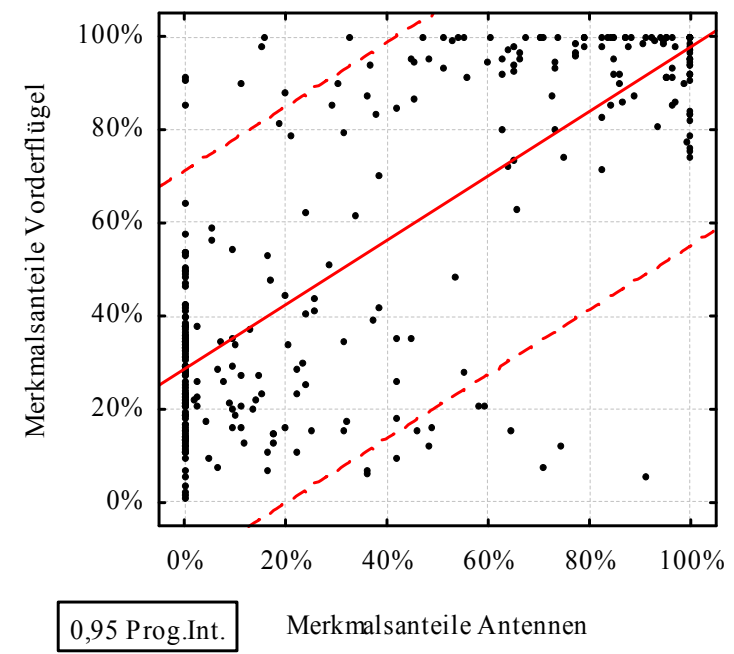

Abb. 77 Korrelationsanalyse der Merkmalsanteile der Vorderflügel- und Antennenmerkmale bei allen Weibchen und Weibchen des Tomaros. Regressionsgerade (-) und Regressionsbänder (- -) (Prognostiziertes Intervall: Niveau 0,95 )

\subsection{Gesangsanalyse}

In diesem Kapitel wird der Balzgesang der Heuschrecken beider Referenzpopulationen und jener des Tomaros beschrieben. Zur Charakterisierung der Tiere werden acht Gesangsmerkmale herangezogen, in denen sich die beiden Arten signifikant voneinander unterscheiden. Im Anschluss daran werden mit der Hauptkomponentenanalyse (Kapitel 2.4.3) die Merkmale für jedes Tier zusammengefasst, so dass den Individuen Hybridindices zugeteilt werden können.

\subsubsection{Werbeverhalten der Männchen von Stenobothrus rubicundus}

Die Männchen führen in Gegenwart eines Weibchens eine Balz, bestehend aus drei unterschiedlichen Phasen, auf. Dabei erzeugen die Männchen Laute durch Stridulation der Hinterbeine an den Vorderflügeln und durch Aneinanderschlagen der Hinterflügel (Elsner 1974b). Hierbei unterscheiden sich im Mittelteil der Balz die Männchen der alpindalmatinischen Populationen von denen der griechischen (Elsner \& Wasser (1995a, b, c).

In jedem Falle beginnt der Werbegesang (Abb. 78) mit einer in der Länge sehr variablen, von wenigen Sekunden bis hin zu einer halben Stunde andauernden Stridulation (Phase I). Hierbei werden die Laute ausschließlich durch Stridulation der Hinterbeine an den Vorder- 
flügeln durch Auf- und Abwärtsbewegung erzeugt. Daran schließt sich die Phase II an, für die bei den griechischen Populationen vor allem der Wechsel zwischen Beinbewegungen, die der Phase I ähneln, und kurzen lauthaften Flügelschlägen charakteristisch ist. Die Männchen der alpin-dalmatinischen St. rubicundus-Population schlagen an diesen Stellen nicht mit den Flügeln, sondern führen stufenartige Abwärtsbewegungen mit den Hinterbeinen aus. Trotz der unterschiedlichen Art der Stridulation - einmal mit den Flügeln, das andere Mal mit den Beinen - ähneln sich die erzeugten Lautmuster sehr.

Bei den Männchen aller Populationen geht die Phase II nahtlos in eine kontinuierliche, bis zu $30 \mathrm{~s}$ anhaltende lauthafte Flügelstridulation über (Phase III). Es kommt nicht selten vor, dass das St. rubicundus Männchen dabei vom Boden abhebt und versucht in der Nähe des Weibchens zu landen, um so einen Begattungsversuch zu starten.

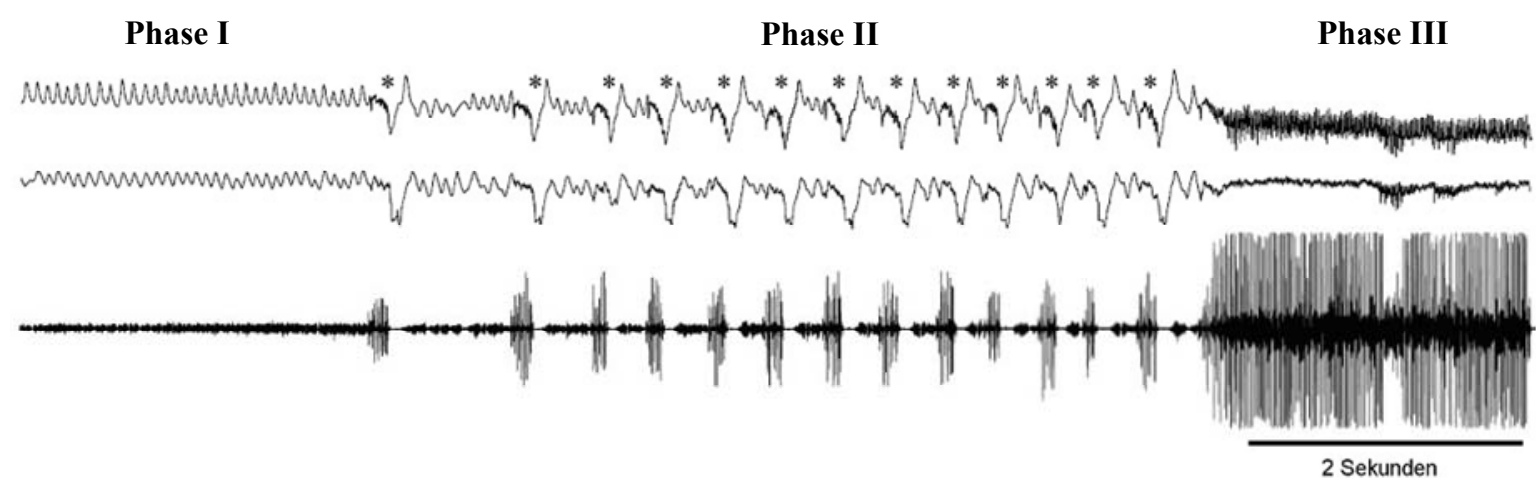

Abb. 78 Werbegesang von St. rubicundus Männchen. Bewegungsspur des linken (oben) und des rechten (Mitte) Hinterbeins, sowie die dazugehörige Tonspur (unten). Flügelschlag (*).

\subsubsection{Das Werbeverhalten der Männchen von Stenobothrus clavatus}

Der Balzgesang der Männchen von St. clavatus besteht, anders als der Gesang der Männchen von St. rubicundus aus vier Gesangsphasen (Abb. 78). Die Männchen dieser Art verwenden für die Stridulation ausschließlich ihre Hinterbeine, die sie an ihre Vorderflügel reiben, sie schlagen also nicht mit den Flügeln (Sradnick 2006, Ostrowski et al. 2009).

Die Balz beginnt mit einem leisen, bis zu 45 min dauernden Eingangsteil (Phase I), die aus einfachen Auf- und Abwärtsbewegungen der Hinterbeine zusammengesetzt ist. Nur die Abstriche sind lauthaft, die Aufwärtsbewegungen hingegen bleiben stets stumm. 
An Phase I schließt sich die kurze (10 s bis $20 \mathrm{~s}$ ) und sehr leise Phase II an, die aus schnell aufeinander folgenden, lauthaften Auf- und Abbewegungen (Länge ca. 100 ms) besteht. Gleichzeitig mit diesen schnellen Beinbewegungen schwingen die Männchen ihren Körper ruckartig nach links und rechts.

Im Anschluss an Phase II folgen bis zu 25 großamplitudige und komplexe Beinbewegungen (Phase III). Hierbei werfen die Männchen sowohl ihre Hinterbeine, als auch ihre Antennen nach vorne, und es werden Ansprungversuche in Richtung der Weibchen unternommen. Auf- und Abbewegung sind stets lauthaft, wobei die Abwärtsbewegung im ersten Drittel eine vibratorische Modulation von ca. $80 \mathrm{~Hz}$ aufweist. Das letzte Drittel besteht aus einer lauten stufigen Abwärtsbewegung, die aus 7 bis 13 Lautpulsen besteht. Im mittleren Drittel wird kein Laut produziert. Sind die mit dieser Phase verbundenen Anspringversuche erfolgreich, kommt es zur Kopulation. Wehrt das Weibchen hingegen diese Versuche $\mathrm{ab}$ oder entfernt sich vom Männchen folgt die nur 2-3 s dauernde Phase IV.

In ihr werden ca. 150 bis 250 ms lange Auf- und Abbewegungen durchgeführt. Die Aufwärtsbewegung ist stumm und die Abwärtsbewegung wird stufenartig durchgeführt. Diese Bewegung ähnelt stark der Bewegung im letzten Drittel der Abwärtsbewegung in der Phase III.

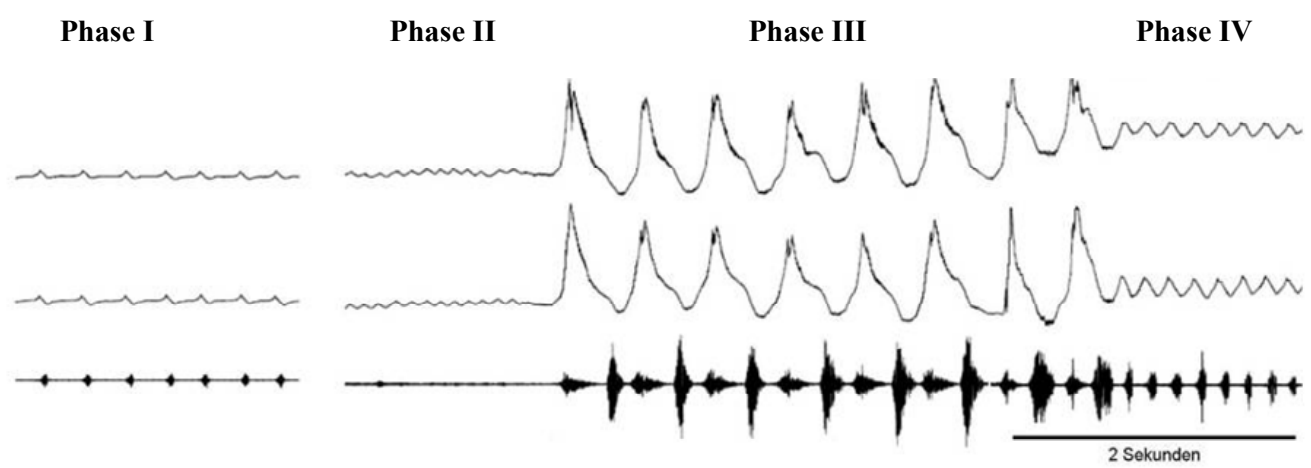

Abb. 79 Gesang von St. clavatus Männchen. Bewegungsspur des linken (oben) und des rechten (Mitte) Hinterbeins sowie die dazugehörende Tonspur (unten).

\subsubsection{Werbeverhalten der Tomaros-Heuschrecken}

Von Sradnick (2006) sind bereits einige der Tomaros-Heuschrecken, die in ihrer Balz intermediäre Gesangselemente aufweisen, beschrieben worden. Neben Tieren, deren Gesang dem der Arten St. rubicundus bzw. St. clavatus sehr ähnlich ist, gibt es auch Tiere, die Gesangselemente beider Arten in ihrem Werbeverhalten präsentieren. Sogar neue Gesangs- 
elemente, bei denen Teile der elterlichen Gesänge neu kombiniert werden, so dass ein völlig neues Element entsteht, sind in einigen Fällen zu beobachten und durchaus nicht selten. In der folgenden Abbildung werden einige intermediäre Gesangselemente (Abb. $80 \mathrm{~B}$ ) im Vergleich zu den Elementen der Arten St. clavatus Abb. 80 A) und Abb. 80 C) St. rubicundus dargestellt. Intermediäre Heuschreckengesänge können sich aus Elementen beider Elternarten zusammensetzen. Diese können in ihrer parentalen Form aneinander gereiht werden oder in sich vollkommen neu sein. Bei diesen Gesangsmustern werden z. B. im Wechsel stufige Abwärtsbewegungen und Flügelschläge präsentiert. Die Abbildung zeigt nur einige Beispiel der gesamten Variationsbreite.

A
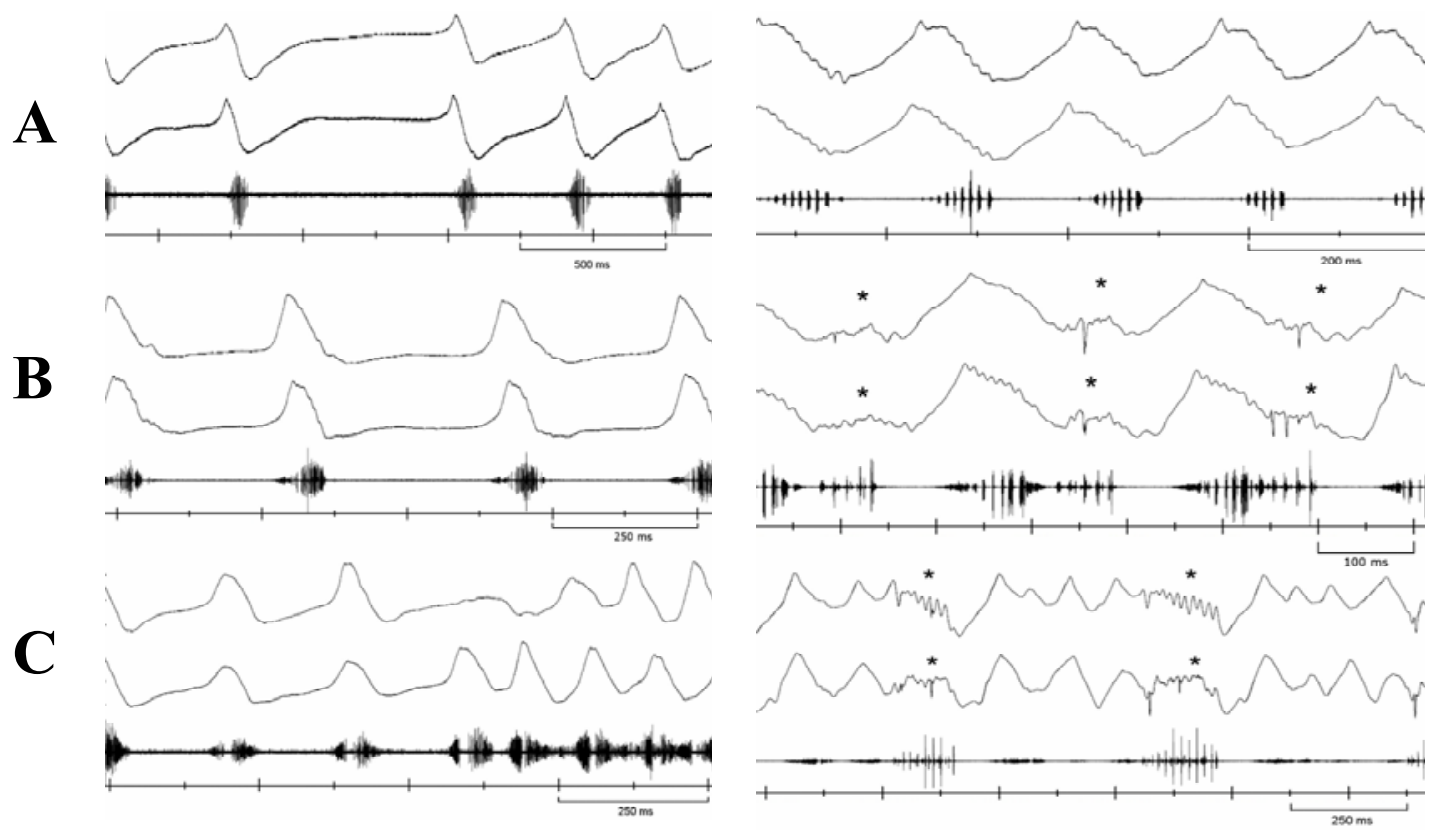

Abb. 80 Beispiele für intermediäre Gesangsmerkmale (Mitte) im Vergleich zu Gesangsphasen der Art St. clavatus (oben) und St. rubicundus (unten). Bewegungsspur des linken (oben) und des rechten (Mitte) Hinterbeins, sowie die dazugehörige Tonspur (unten). Flügelschlag $\left({ }^{*}\right)$

\subsubsection{Beschreibung der Gesangsmerkmale}

Um die Gesänge der beiden Arten und die ihrer Zwischenformen (Hybriden) zu charakterisieren, wurden acht Merkmale herangezogen, die im Folgenden beschrieben werden. In diesem Zusammenhang wurden die Individuen entsprechend ihren Fundorten in fünf Gruppen eingeteilt: (1) St. clavatus ( $\mathrm{n}=13)$ vom Xerovouni, (2) St. rubicundus $(\mathrm{n}=12)$ aus den allopatrischen griechischen Populationen, (3) und (4) Tomaros-Heuschrecken außerhalb (mind. $1 \mathrm{~km})$ der Kontaktzonen, die mehrheitlich St. rubicundus $(\mathrm{n}=18)$ bzw. St. clavatus ( $\mathrm{n}=26)$ ähneln sowie (5) Individuen in unmittelbarer Nähe (ca. $200 \mathrm{~m}$ zu beiden Seiten) der Kontaktzonen. 
Im Folgenden werden die Ausprägungen der einzelnen Merkmale in den Gruppen genauer beschrieben und mittels ANOVA/Scheffé miteinander verglichen.

\subsubsection{Merkmal 1 - Lautstärkenverhältnis der Auf- und Abwärtsbewegungen in Phase I}

Wie oben beschrieben, beginnen die Werbegesänge sowohl von St. clavatus als auch bei St. rubicundus aus Folgen von einfachen Auf- und Abwärtsbewegungen (Silben), bei denen entweder beide Bewegungsrichtungen (St. rubicundus) oder nur die Abwärtsbewegungen (St. clavatus) lauthaft sind. Es wurde das Verhältnis der maximalen Amplitude (in $\mathrm{mV}$ ) des während der Aufwärtsbewegung erzeugten Lautpulses und der maximalen Amplitude des Pulses während der Abwärtsbewegung bestimmt (Abb. 81). Bei jedem Individuum wurden insgesamt 10 bis 15 Auf- und Abwärtsbewegungen gemessen und schließlich wurde zur Normierung der errechneten Verhältnisse der logarithmierte Mittelwert gebildet (Merkmal 1).

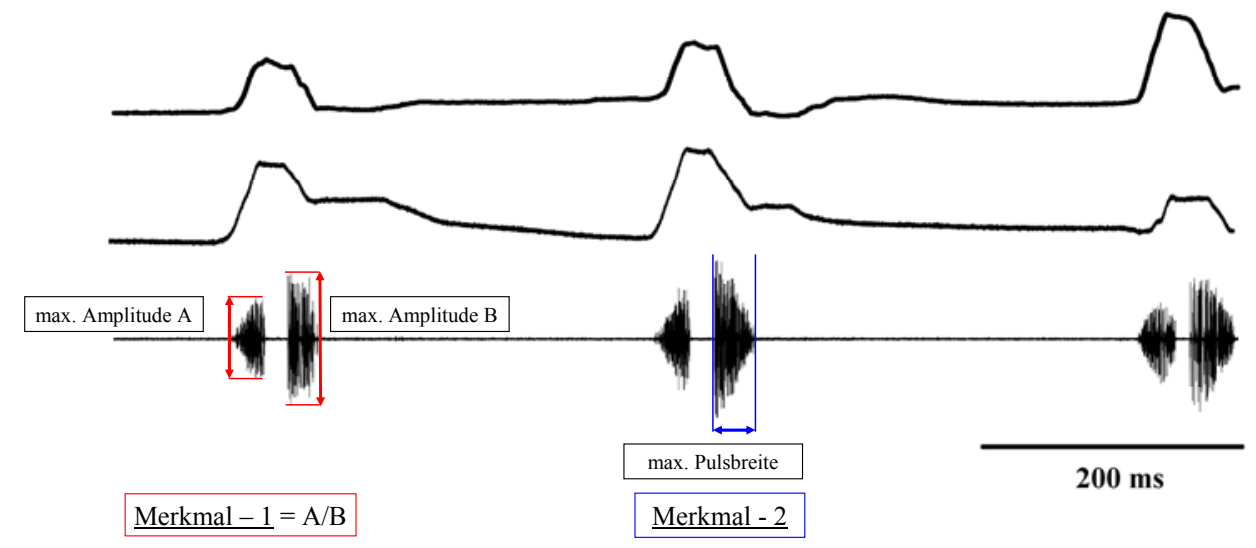

Abb. 81 Beispiel für die Bestimmung des Merkmals 1 (rot) und Merkmals 2 (blau)

Bei Individuen der Art St. rubicundus sind Auf- und Abwärtsbewegung lauthaft und das durchschnittliche Verhältnis beider Lautpulse liegt bei den Individuen der griechischen Individuen bei durchschnittlich 0,73 $(\sigma=0,15)$. Somit ist die Amplitude (Lautstärke), die bei der Abwärtsbewegung erzeugt wird, etwas größer als die der Aufwärtsbewegung. Bei Individuen der Art St. clavatus hingegen ist die Aufwärtsbewegung völlig stumm, das Verhältnis Lautstärke Aufwärtsbewegung/Lautstärke Abwärtsbewegung liegt infolgedessen bei Null und ist somit signifikant $(\mathrm{p}<0,001)$ verschieden (ANOVA/Scheffé). Die St. rubicundus des Tomaros zeigen bei diesem Merkmal ein durchschnittliches Amplituden Verhältnis von $0,69(\sigma=0,19)$ und haben somit ein etwas kleineres Verhältnis als die der Refe- 
renzpopulationen. Der ANOVA-Test ergab aber keinen signifikanten Unterschied zu der St. rubicundus Referenzgruppe. Auch die St. clavatus des Tomaros unterscheiden sich mit einem Amplitudenverhältnis von 0,08 $(\sigma=0,09)$ etwas, aber nicht signifikant von denen des Xerovouni. Individuen der Kontaktzone mit einem durchschnittlichen Verhältnis von 0,37 $(\sigma=0,25)$ liegen zwischen den Elternarten und unterscheiden sich signifikant $(p<0,001)$ von den anderen Populationen. In Abb. 82 wird aber auch deutlich, dass die Individuen der Kontaktzonen teilweise einer der beiden Elternarten entsprechen, aber auch intermediäre Merkmalszüge aufweisen.

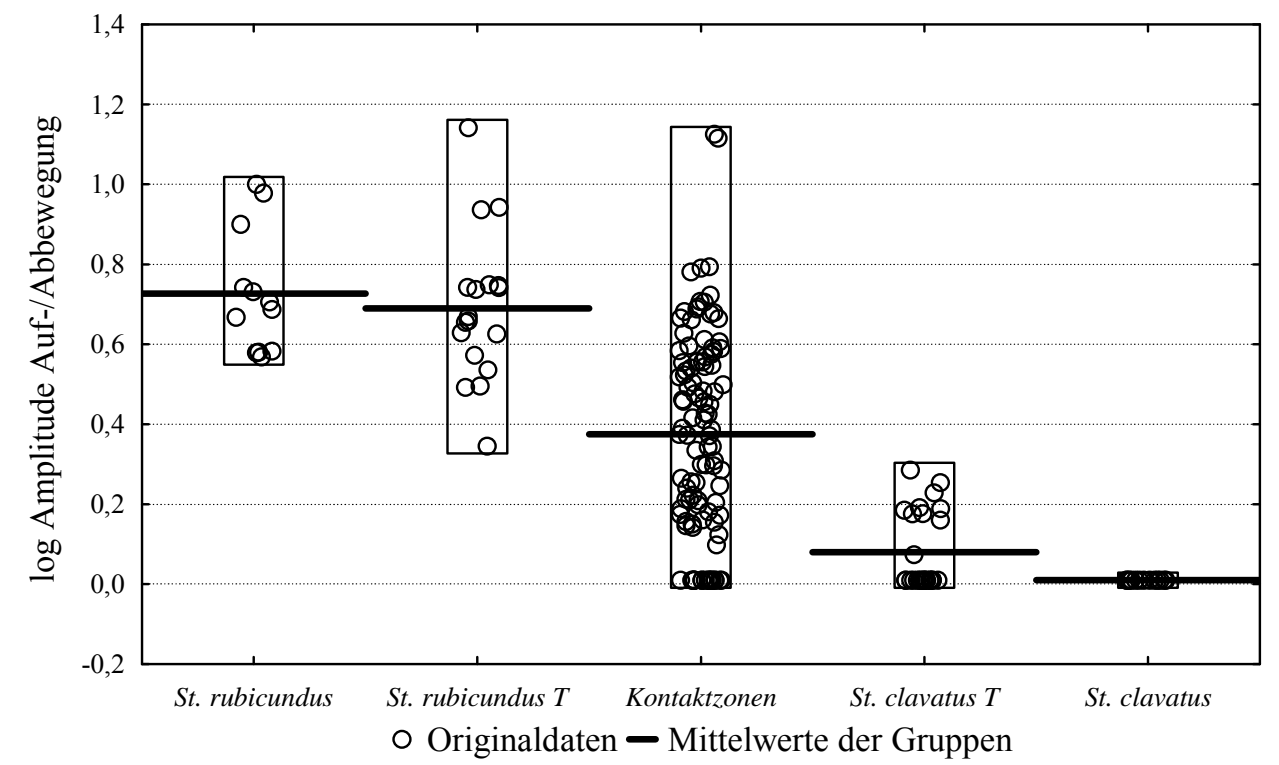

Abb. 82 Streuungsplot des Verhältnisses der Amplituden von Aufstrich- und Abstrichpulsen in Phase I (Verhaltensmerkmal 1) mit den entsprechenden Mittelwerten der Gruppen

\subsubsection{Merkmal 2 - Pulsdauer während der Abwärtsbewegung in Phase I}

Bei diesem Gesangsmerkmal wird die durchschnittliche Pulsdauer der Abwärtsbewegung in der Phase I des Männchengesanges bestimmt. Hierzu wurden mindestens 10 bis 15 dieser Merkmale pro Individuum vermessen (Abb. 81).

Wie in Abb. $83 \mathrm{zu}$ sehen ist, liegt die Pulsdauer bei den Vertretern der Art St. rubicundus bei durchschnittlich $43 \mathrm{~ms}(\sigma=12)$ und ist somit signifikant kürzer als die durchschnittlich 70 ms $(\sigma=12)$ andauernde Abwärtsbewegung bei den St. clavatus-Männchen. Die St. rubicundus-Individuen des Tomaros haben bei diesem Merkmal eine durchschnittliche Pulsdauer von $51 \mathrm{~ms}(\sigma=12)$ und zeigen somit einen etwas längeren, jedoch nicht signifikant verschiedenen Abwärtspuls, als die der St. rubicundus-Männchen der anderen Populatio- 
nen. Die St. clavatus des Tomaros haben im Vergleich zu denen des Xerovouni mit $64 \mathrm{~ms}$ $(\sigma=8)$ eine etwas kürzere, aber nicht signifikant verschiedene Abwärtsbewegung. Zwischen den St. clavatus und den St. rubicundus liegen bei diesem Merkmal mit durchschnittlich 55 ms $(\sigma=13)$ die Individuen der Kontaktzonen. Wie auch bei Merkmal 1 unterscheiden sich die Individuen der Kontaktzone signifikant von den anderen Populationen.

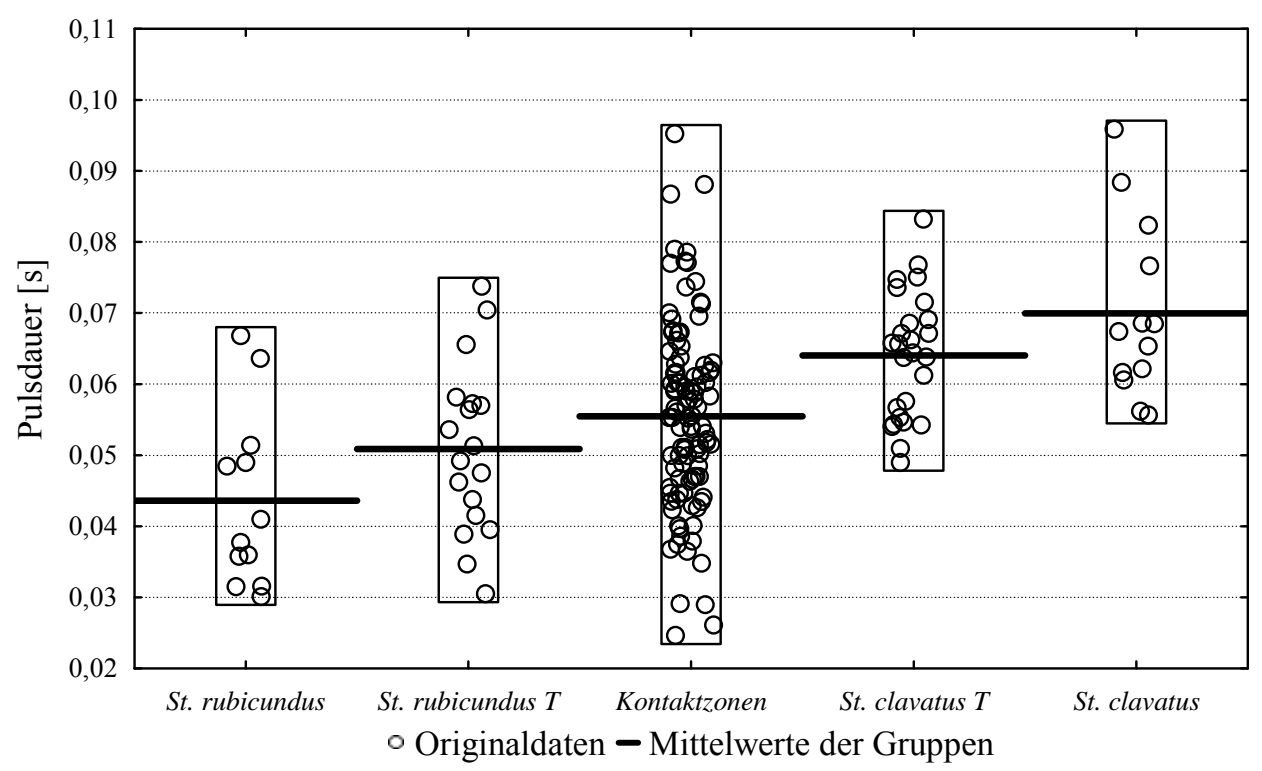

Abb. 83 Streuungsplot der Dauer der Abstrichpulse in Phase I (Verhaltensmerkmal 2) mit den entsprechenden Mittelwerten der Gruppen

\subsubsection{Merkmal 3 - Anzahl der Flügelschläge in Phase II}

Für dieses Merkmal wurde die durchschnittliche Anzahl pro Gesang eines Individuums, an kurzen Flügelschlägen, die in Phase II zwischen lauthaften Auf- und Abwärtsbewegungen der Hinterbeine eingestreut werden, ermittelt. 


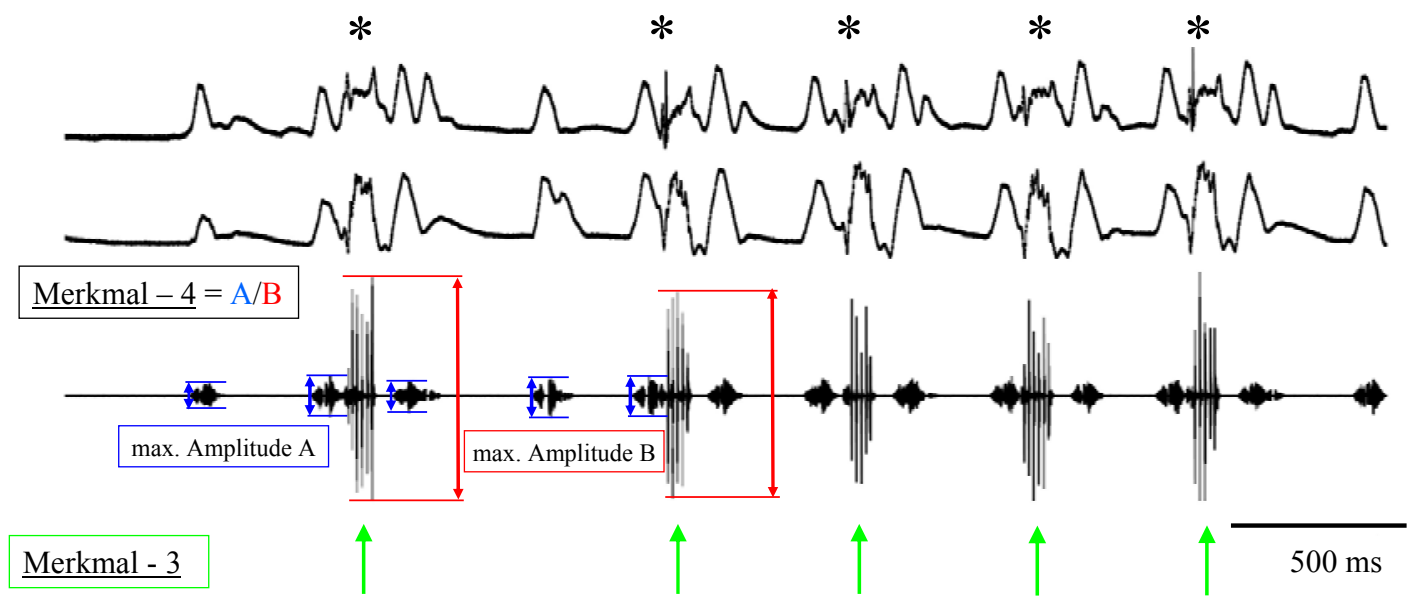

Abb. 84 Phase II eines griechischen St. rubicundus Männchen. Bewegungsspur des linken (oben) und des rechten (Mitte) Hinterbeins, sowie die dazugehörige Tonspur (unten). Flügelschlag (*). Beispiel für die Bestimmung des Merkmals 3 (grün) und Merkmals 2 (blau bzw. rot)

Individuen der Art St. rubicundus führen hier durchschnittlich 46,90 $(\sigma=31,43)$ Flügelschläge aus, wobei dieser Wert aber durchaus zwischen 7 und 100 Flügelschlägen variieren kann (Abb. 85). Bei Individuen der Art St. clavatus des Xerovouni und des Tomaros hingegen werden keine Flügelschläge in dieser Phase beobachtet. Diese unterscheiden sich somit signifikant von den Individuen der Art St. rubicundus. Die St. rubicundus des Tomaros schlagen in Phase II durchschnittlich 29,96-mal $(\sigma=29,18)$ und somit seltener mit den Flügeln, als die der allopatrischen Populationen. Dieser Unterschied ist aber nicht signifikant. Individuen der Kontaktzonen schlagen im Mittel 10,35-mal in Phase II mit den Flügeln, aber ebenso wie bei St. rubicundus zeigt dieses Merkmal auch bei den Individuen des Tomaros eine große Standardabweichung $(\sigma=22,98)$ und unterscheidet sich stark $(p>0,001)$ von den anderen Populationen. 


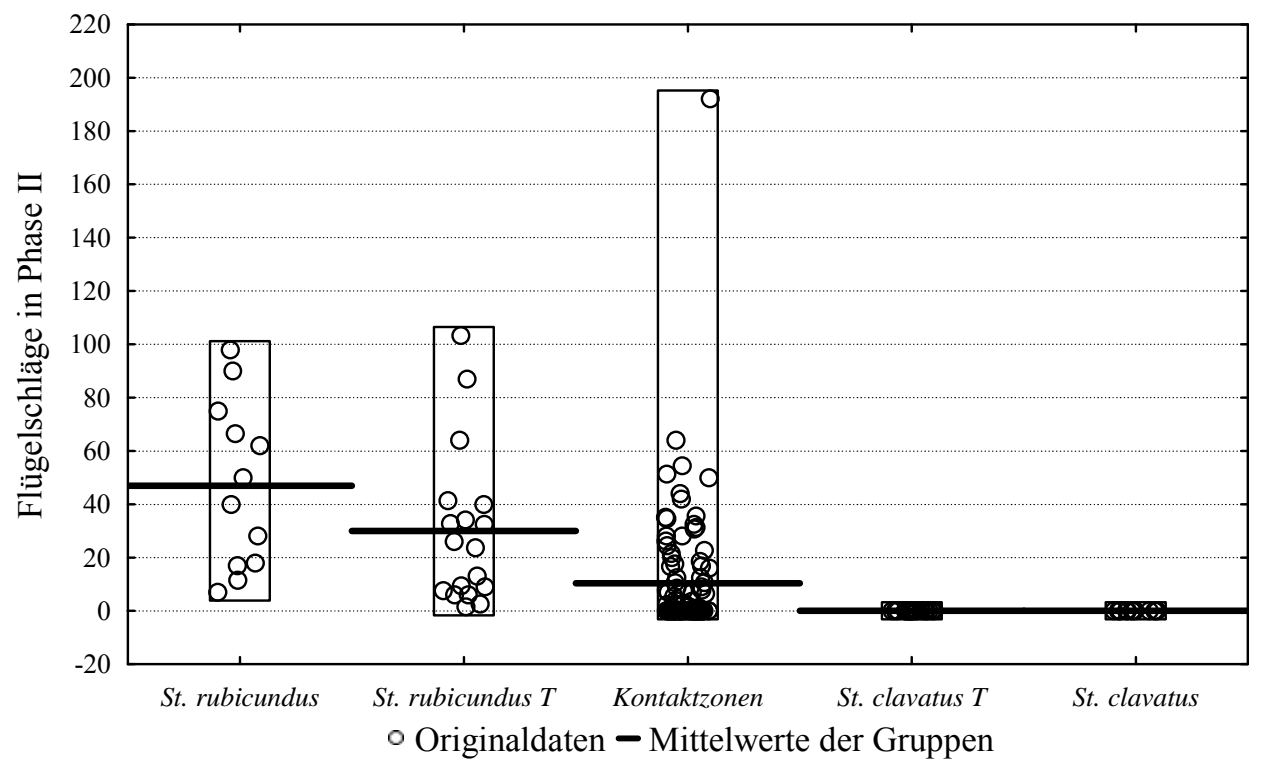

Abb. 85 Streuungsplot der Zahl der Flügelschläge in Phase II (Verhaltensmerkmal 3) mit den entsprechenden Mittelwerten der Gruppen

\subsubsection{Merkmal 4 - Verhältnis von Bein/Flügel -Pulsamplitude}

Als weiteres Merkmal zur Charakterisierung der Gesänge dient das Verhältnis der Amplituden der während der Bein- bzw. Flügelschläge erzeugten Lautpulse (Abb. 84). Hierbei werden im Durchschnitt jeweils 10 bis 15 Pulse ausgewertet. Bei der Hinterbeinstridulation sind beide Bewegungsrichtungen lauthaft; für die Messung herangezogen wurde stets der beim Beinabschlag erzeugte Puls. Wenn in einem Balzgesang keine Flügelschläge dargeboten wurden, wird dem Individuum für dieses Verhältnis ein fiktiver Wert von 10 zugeteilt (siehe Vedenina 2010). Dieser Wert wird so gewählt, dass er möglichst groß ist und somit keine Überschneidungen zulässt.

Wie auch bei Merkmal 2 wird für weiterführende Berechnungen, zwecks Normierung, der logarithmierte Mittelwert aus diesen Daten berechnet. Das durchschnittliche Verhältnis der Pulsamplituden der Individuen (Abb. 86) der Art St. rubicundus liegt bei 0,34 $(\sigma=0,18)$, denn die Amplitude der Flügelschläge ist um etwa ein Drittel höher als die der Beinbewegungen. Alle St. clavatus (Xerovouni und Tomaros) zeigen bei den Verhaltensaufzeichnungen keinen Flügelschlag. Insgesamt zeigen die St. rubicundus des Tomaros durchschnittlich ein Verhältnis von $0,24(\sigma=0,11)$ in Phase II. Sie unterscheiden sich daher nicht signifikant von den allopatrischen St. rubicundus-Populationen. Das durchschnittliche Verhältnis liegt bei den Individuen der Kontaktzonen bei 5,55 $(\sigma=4,78)$. Dies liegt vor allem daran, dass von den 105 Individuen dieser Gruppe 53\% (n=56) keinen Flügelschlag in 
dieser Phase zeigen, die anderen Individuen haben hingegen ein durchschnittliches Amplitudenverhältnis von $0,47(\sigma=0,50)$. Insgesamt unterscheiden sich die Individuen der Kontaktzone aber signifikant von den anderen Populationen.

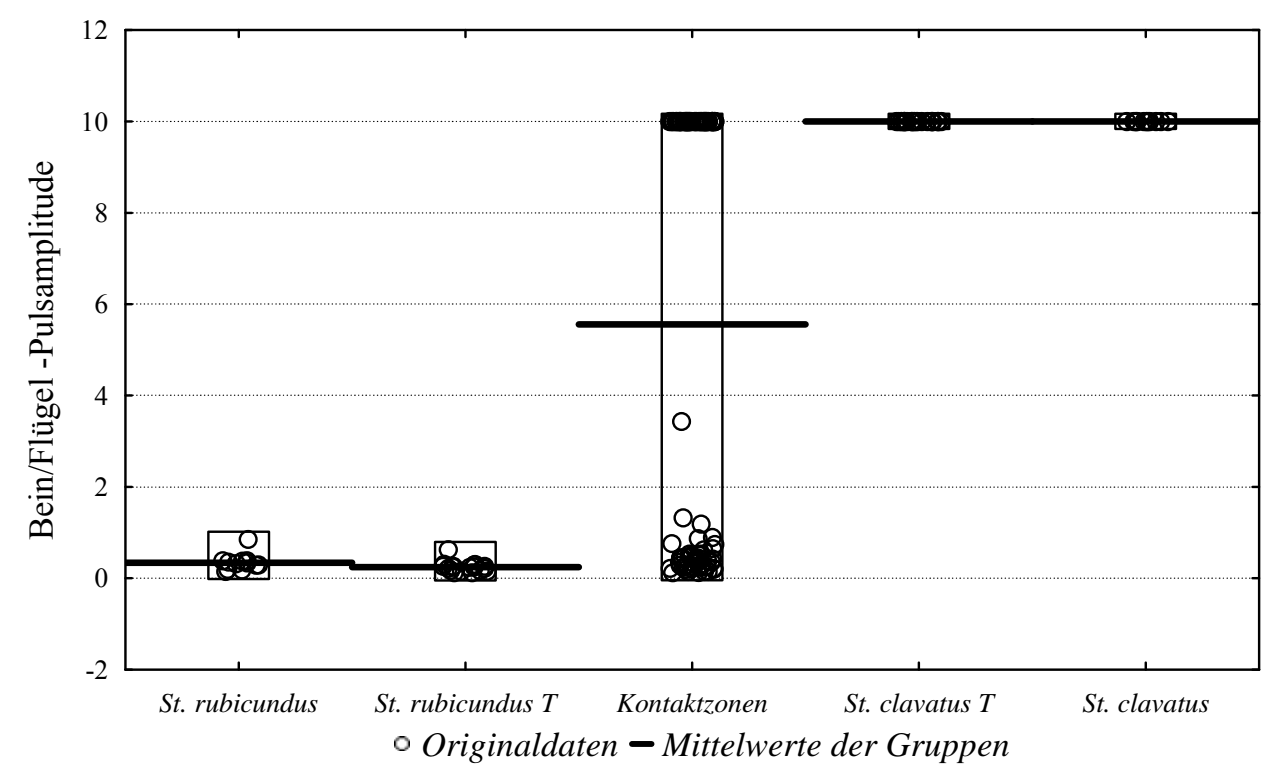

Abb. 86 Streuungsplot des Verhältnisses der Lautamplituden bei Bein- und Flügelstridulation (Verhaltensmerkmal 4) mit den entsprechenden Mittelwerten der Gruppen.

\subsubsection{Merkmal 5 - Einfache Lautpulse im Verhältnis zu mehrfachpulsierten Lau- ten in Phase II}

Ein auffälliges Merkmal in den Gesängen von St. rubicundus und St. clavatus sind mehrfachpulsierte Lautblöcke, die entweder durch kurze Folgen schneller Flügelschläge oder durch stufenartige Abwärtsbewegungen der Hinterbeine erzeugt werden. Zwischen ihnen werden, beispielsweise in Phase II des Gesangs von St. rubicundus, geschlossene Lautpulse eingeschaltet, die durch einfache Auf-/Ab-Bewegungen der Hinterbeine hervorgebracht werden (Abb. 10). Das Verhältnis der Zahl dieser Einfachpulse zu den mehrfachpulsierten wird ebenfalls zur Charakterisierung der Gesänge herangezogen (Merkmal 5). Zeigen Individuen keine mehrfachpulsierten Abwärtsbewegungen oder Flügelschläge, so wird diesen Tieren ein Wert von 0,01 für dieses Merkmal zugeordnet. Dies ist nötig, da ein Wert von Null nicht logarithmiert werden kann. 


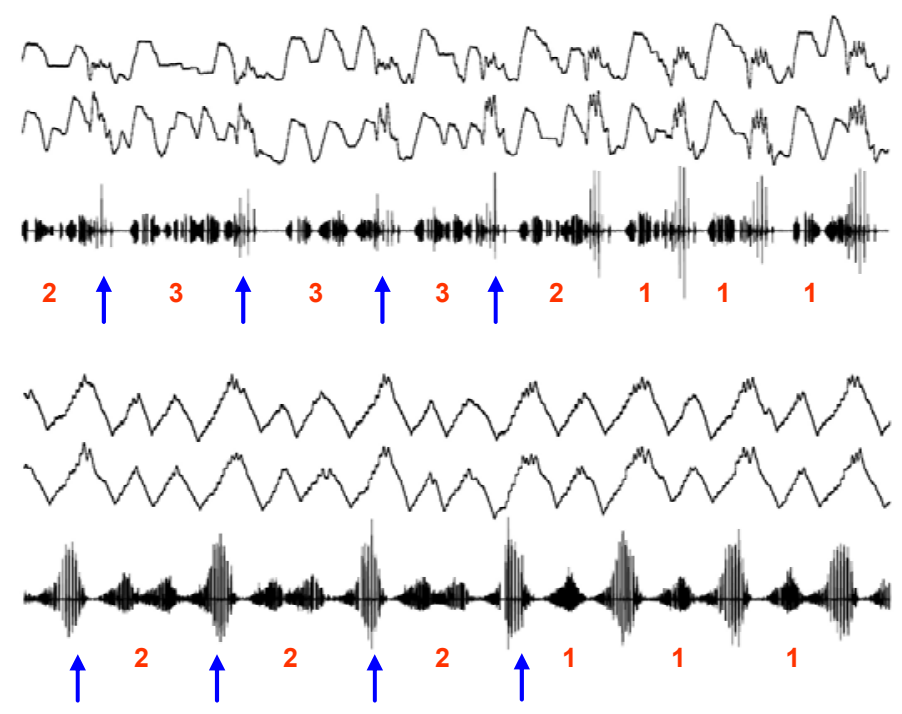

Abb. 87 Beispiel des Merkmals 5 bei drei verschiedenen Individuen. Rote Zahlen: Anzahl der Silben des Typ A. Blaue Pfeile: Mehrfachpulsierte Abwärtsbewegungen oder Flügelschläge.

Für die Tiere der Art St. rubicundus ist ein Verhältnis von 2,92 $(\sigma=1,11)$ für das Merkmal 5 ermittelt worden. Mit anderen Worten: Auf jede dritte Silbe, die durch eine einfache Beinbewegung erzeugt wurde, kommt eine Flügelschlagsfolge. Bei St. rubicundus des Tomaros kommen auf eine Mehrfachpuls-Silbe in Phase II des Balzgesangs durchschnittlich 2,87 Silben $(\sigma=2,09)$. Es ist aber zu beachten, dass diese Individuen im Vergleich zu den Tieren der allopatrischen Populationen eine größere Standardabweichung aufweisen. In Gegensatz zu den St. clavatus des Xerovouni zeigen nicht alle St. clavatus des Tomaros keine stufigen Abwärtsbewegungen in Phase II. Von den 26 hier untersuchten Individuen führten zwei stufige Abwärtsbewegungen aus und tragen daher zum veränderten Verhältnis von 0,56 $(\sigma=2,02)$ bei, wobei dieser Unterschied aber nicht signifikant ist. Abschließend wurden die Tiere der Kontaktzonen betrachtet, die mit einem Verhältnis von durchschnittlich 1,39 $(\sigma=1,72)$ zwischen den Arten St. rubicundus und St. clavatus liegen und signifikante Unterschiede zu den anderen Populationen zeigen. 


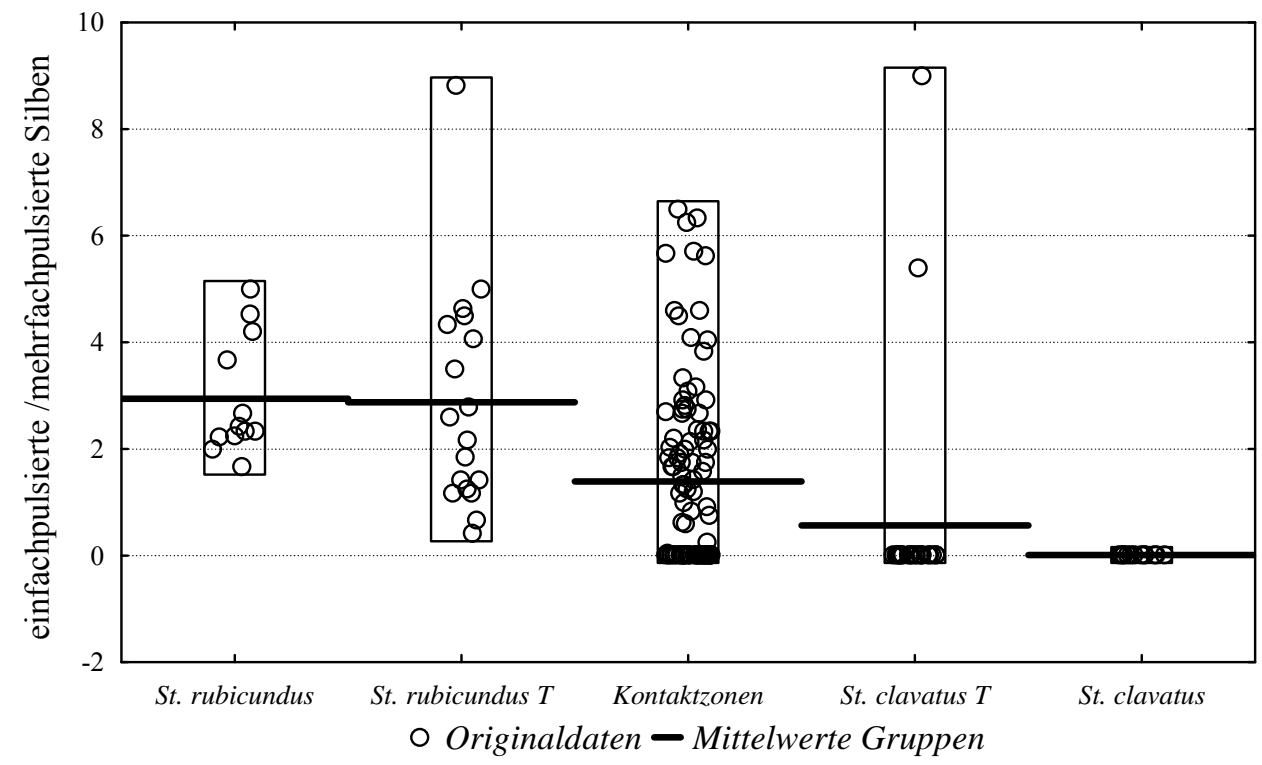

Abb. 88 Streuungsplot des Verhältnisses der einfachen und der mehrfachpulsierten Silben (Verhaltensmerkmal 5) mit den entsprechenden Mittelwerten der Gruppen

\subsubsection{Merkmal 6 - Dauer des anhaltenden Flügelschlags am Ende der Balz}

Ein weiteres Merkmal zur Charakterisierung des Gesangs von St. rubicundus ist die Länge des anhaltenden Flügelschlages (in Millisekunden). Als lang anhaltend werden Flügelschläge definiert, die länger als $250 \mathrm{~ms}$ sind (Beispiel Abb. 89 blau).

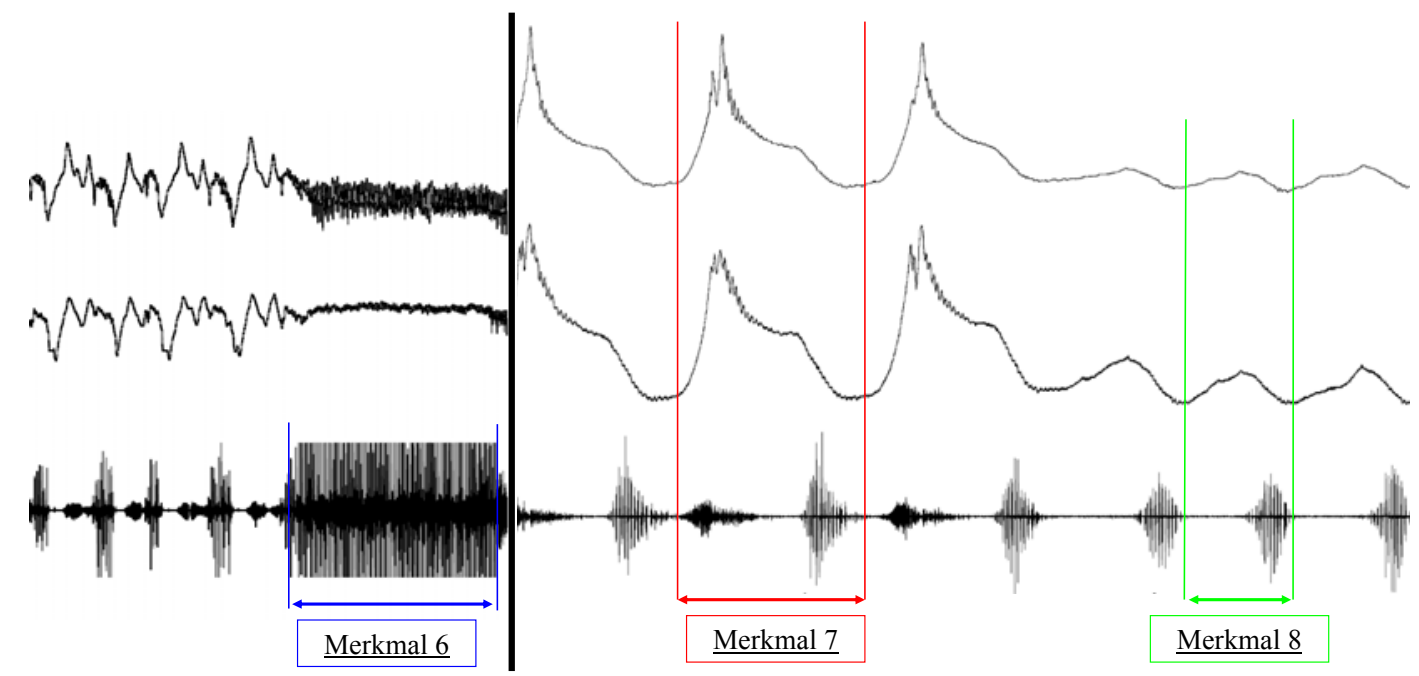

Abb. 89 Beispiele für Merkmal 6 (blau). Ausschnitt aus einem St. rubicundus-Gesang. Beispiel für Merkmal 7 (rot) und Merkmal 8 (grün) aus einem St. clavatus-Gesang. Bewegungsspur des linken (oben) und des rechten (Mitte) Hinterbeins, sowie die dazugehörige Tonspur (unten). Flügelschlag (*)

Bei den Individuen der allopatrischen St. rubicundus-Populationen Griechenlands ist der Flügelschlag durchschnittlich 8,45 Sekunden lang. Aber eine Standardabweichung von $\sigma=13,97$ zeigt, dass dieses Merkmal sehr variabel ist (Abb. 90). Bei den St. rubicundus des 
Tomaros ist mit durchschnittlich 5,09s $(\sigma=5,49)$ ein etwas kürzerer Flügelschlag zu beobachten als bei den Tieren der allopatrischen St. rubicundus-Population. Der Unterschied ist aber nicht signifikant. Schaut man sich aber die Werteverteilung in Abb. 90 an, relativiert sich dieser Unterschied, welcher bei der Art St. rubicundus durch einen Ausreißer zu Stande gekommen ist. In unmittelbarer Nähe zu den Kontaktzonen schlagen die Individuen durchschnittlich für 0,95 $(\sigma=1,87)$ Sekunden mit den Flügeln. Aber von den 105 Individuen schlagen 64 (61\%) wie auch St. clavatus in Phase III gar nicht mit den Flügeln. Anders als bei St. rubicundus zeigen die Vertreter der Art St. clavatus des Xerovouni, aber auch die des Tomaros keinen anhaltenden Flügelschlag.

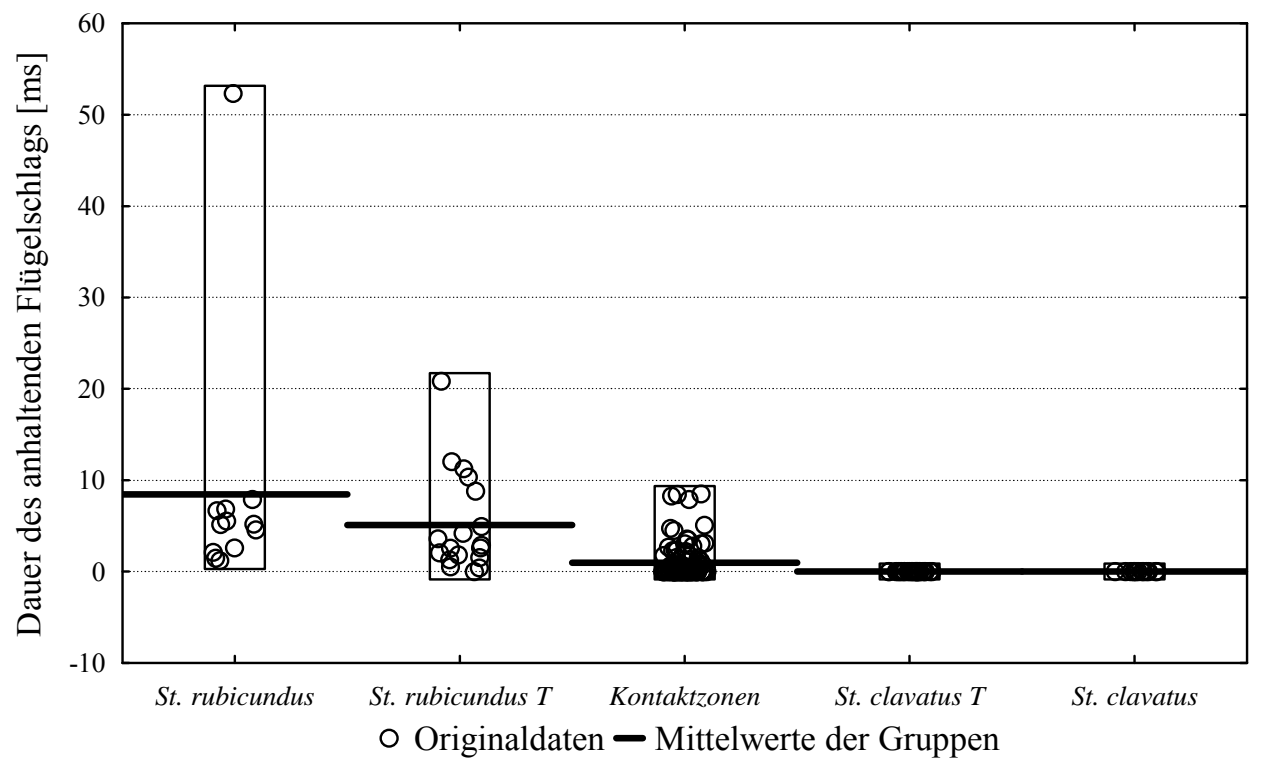

Abb. 90 Streuungsplot der Länge der Flügelstridulation (Verhaltensmerkmal 6) mit den entsprechenden Mittelwerten der Gruppen.

\subsubsection{Merkmal 7 - Anzahl der komplexen, hochamplitudigen Beinbewegungen}

Bei diesem Merkmal handelt es sich um die durchschnittliche Anzahl der hochamplitudigen und sehr komplexen Beinbewegungen wie sie für Phase III der St. clavatus-Balz typisch sind. Die Aufwärtsbewegung ist lauthaft, und auf diese folgen nach einer kurzen Pause einige Schwirrlaute die schließlich in eine laute stufige Abwärtsbewegung übergehen (Abb. 89 rot). Es werden nur Bewegungen gezählt, die auch eine für diese Phase charakteristische Pulsstruktur aufweisen.

Dieses Merkmal ist bei den Vertretern der Art St. rubicundus nicht zu finden. Bei der Art St. clavatus sind durchschnittlich $22,39(\sigma=5,76)$ Auf- und Abwärtsbewegungen gezählt 
worden (Abb. 91). Hingegen wurden bei den St. rubicundus des Tomaros bei einem der 18 Individuen sechs stufige Beinbewegungen gezählt. Insgesamt unterscheiden sich aber diese beiden Populationen nicht signifikant. Bei den Individuen der Art St. clavatus auf dem Tomaros wurden durchschnittlich 29,72 $(\sigma=10,92)$ Beinbewegungen dieses Merkmals gezählt, also etwas mehr als bei den Individuen der allopatrischen Xerovouni-Population, aber nicht signifikant verschieden. Die Gesänge der Individuen der Kontaktzonen beinhalten durchschnittlich 10,34 Bewegungen. Diese sind aber mit einer Standardabweichung von $\sigma=10,66$ sehr variabel. Sie siedeln sich aber auch wie die anderen Merkmale dieser Individuen zwischen den beiden Arten an und unterscheiden sich signifikant von den anderen Populationen.

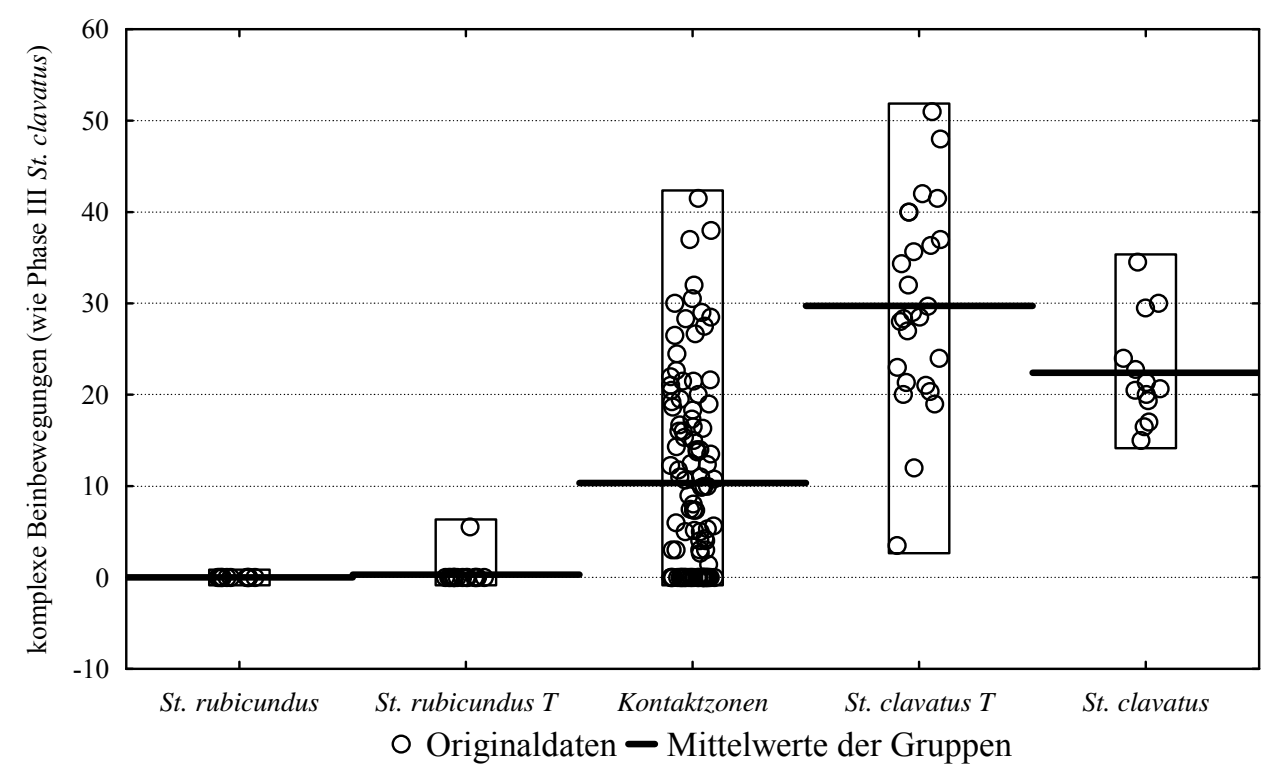

Abb. 91 Streuungsplot des Verhaltensmerkmals 7 mit den entsprechenden Mittelwerten der Gruppen

\subsubsection{Merkmal 8 - Anzahl der Beinbewegungen mit stufiger Abwärtsbewegung}

Grundlage dieses Merkmals ist die durchschnittliche Anzahl der Beinstridulationen mit lauter stufiger Abwärtsbewegung am Ende einer Balz (Abb. 89 grün).

Das letzte der ausgewählten acht Gesangsmerkmale bezieht sich auf die Anzahl der aufeinanderfolgenden (ohne Unterbrechungen) Beinbewegungen, bei der auf eine einfache Aufwärtsbewegung eine laute und stufige Abwärtsbewegung folgt. Wie auch bei Merkmal 7 ist dieses Merkmal bei den Vertretern der Art St. rubicundus nicht zu finden. Drei der 18 St. rubicundus des Tomaros zeigen durchschnittlich 8,5 $(\sigma=5,89)$ Beinbewegungen mit 
stufigen Abwärtsbewegungen. Durchschnittlich zeigen alle St. rubicundus des Tomaros 1,45 $(\sigma=3,83)$ Beinbewegungen. Die Individuen der Art St. clavatus hingegen zeigen durchschnittlich 10,85 $(\sigma=7,17)$ dieser Merkmale in ihrem Balzgesang und so signifikante Unterschiede zu den beiden St. rubicundus Populationen. St. clavatus Männchen des Tomaros führen durchschnittlich 3,77 $(\sigma=3,46)$ stufige Beinbewegungen aus und damit weniger, als die St. clavatus-Männchen des Xerovouni; wobei der Unterschied aber nicht signifikant ist. Mit durchschnittlich 8,97 $(\sigma=14,35)$ Beinbewegungen mit stufiger Abwärtsbewegung ordnen sich die Individuen der Kontaktzone zwischen St. clavatus und St. rubicundus an. Bei einem Drittel (35 Tiere) der 105 Individuen der Kontaktzone ist dieses Merkmal nicht zu finden. Diese Population grenzt sich bei diesem Merkmal signifikant von den anderen Merkmalen ab.

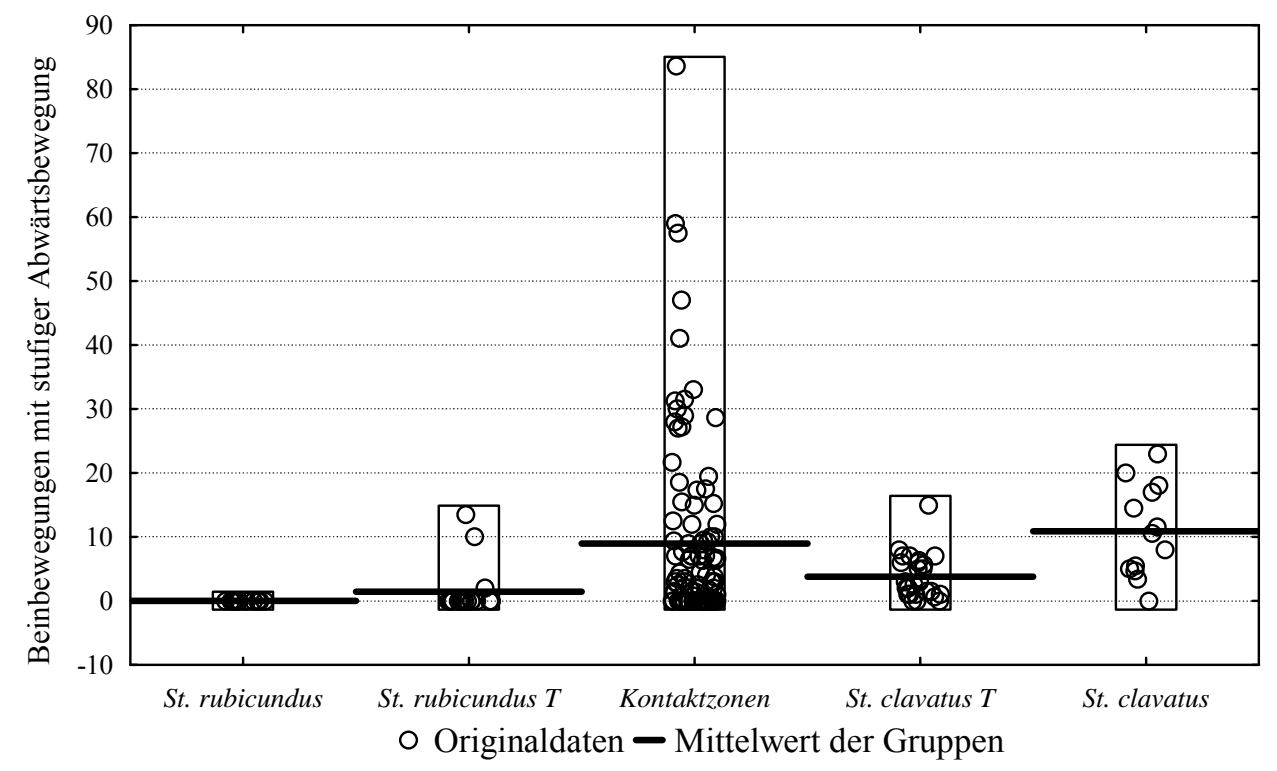

Abb. 92 Streuungsplot des Verhaltensmerkmals 8 mit den Mittelwerten der Gruppen

\section{Zusammenfassung}

Der Merkmalsvergleich zwischen St. rubicundus und St. clavatus zeigt, dass es große und signifikante zwischenartliche Unterschiede gibt (MANOVA: $p<0,0001$ ). Der Wilks Lambda liegt bei 0,015 und spiegelt ebenfalls die großen Gesangsunterschiede wider. Keine signifikanten $(\mathrm{p}>0,05)$ Unterschiede sind zwischen den Arten der allopatrischen ReferenzPopulationen und deren Vertretern des Tomaros (St. clavatus und St. rubicundus von Tomaros) berechnet worden.

Die Individuen der Kontaktzone zeigen eine weitaus größere Variabilität in ihren Merkmalen als die Individuen der Referenzpopulationen. Bei jedem Merkmal sind sowohl Eigen- 
schaften eines der beiden Elternarten als auch intermediäre Eigenschaften gemessen worden. Somit ist bei den Vertretern des Tomaros ein fließender Merkmalsübergang von der einen zur anderen Art zu beobachten. Im Anschluss werden nun die Individuen und ihre Gesangsmerkmale einzeln betrachtet.

\subsubsection{Verhaltensanalyse der einzelnen Individuen}

Um die Unterschiede zwischen den Individuen aufzuzeigen, wurde eine Hauptkomponentenanalyse aller acht Merkmale durchgeführt (Abb. 93). Zur ersten Hauptkomponentenebene (PC1) tragen fast alle der acht Merkmale bei. Allein das Merkmal 2 (Kapitel 3.4.4.2) zeigt einen größeren Beitrag zur Hauptkomponentenebene 2 (PC2), was aber nicht heißt, dass die anderen Merkmale keinen Beitrag zur PC2 leisten würden.

Aus der Hauptkomponentenanalyse (Abb. 93) wird deutlich, wie groß die Merkmalsunterschiede im Balzgesang zwischen St. rubicundus und St. clavatus sind. Beide Arten grenzen sich stark voneinander ab, wobei die Vertreter der Art St. rubicundus etwas breiter streuen, als die der Art St. clavatus. Von den Individuen des Tomaros befinden sich viele Tiere innerhalb der Varianzen einer der beiden Elternarten. Aber der Großteil der Tomaros-Tiere ist bei der Hauptkomponentenanalyse zwischen den beiden Arten zu finden. Die meisten von ihnen gruppieren sich in der Nähe der Art St. clavatus. Etwas weniger Individuen findet man in der Nähe der Art St. rubicundus. Es sind sehr wenige Individuen zu finden, die genau zwischen den beiden Arten stehen, also genau intermediär sind.

Hauptkomponentenanalyse des Verhaltens

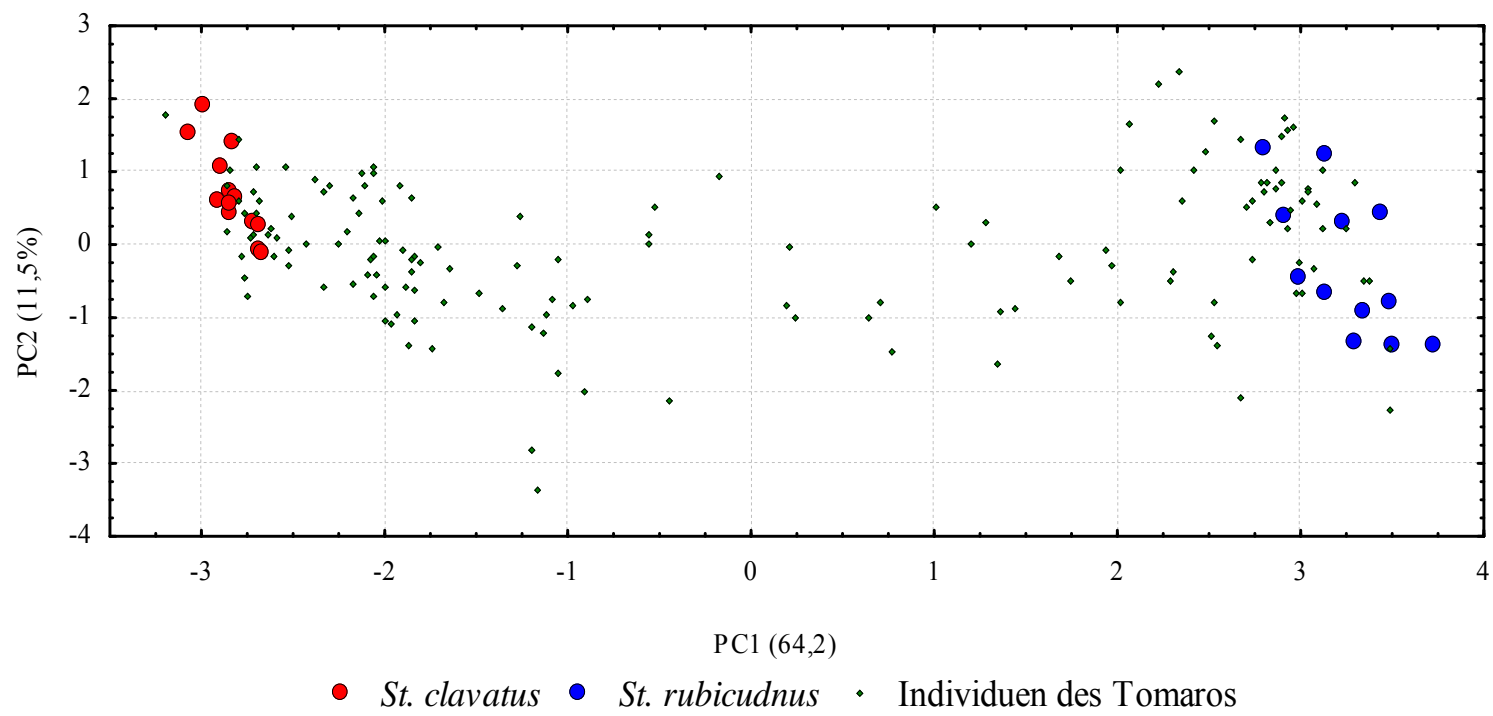

Abb. 93 Hauptkomponentenanalyse der acht Verhaltensmerkmale 
Da die Hauptkomponenten Achse /Ebene 1 (PC1) mit 64,2 \% den größten Anteil an der Gesamtvarianz hat, werden die Werte zur Einordnung der Individuen in das Hybridindexund Merkmalsanteilsystem verwendet. Wie auch bei den Berechnungen der morphologischen Merkmale werden auch hier die Individuen, mit Werten innerhalb der Varianzen der Elternarten, diesen zugeordnet. Sie erhalten somit einen Hybridindex von 1 für St. clavatus und 12 für St. rubicundus. Individuen, die bei der Hauptkomponentenanalyse zwischen den beiden Arten zu finden sind, werden den Hybridindices 2-11 zugeordnet. Des Weiteren wurde, wie auch bei den morphologischen Merkmalen, für jedes Individuum eine Merkmalsverteilung errechnet. Diese umfasst den prozentualen Anteil an St. clavatus bzw. St. rubicundus Merkmalen. Somit hat ein Tier, das der Art St. clavatus entspricht einen Merkmalsanteil von 100\% St. clavatus-Merkmalen und 0\% St. rubicundus-Merkmalen. Intermediäre Individuen haben in Folge dessen Anteile beider Art-Merkmale. Basierend auf dem Merkmalsanteil der Art St. rubicundus werden die durchschnittlichen Merkmalsanteile der Individuen jedes Fundortes (GPS-Punkt) in Abbildung Abb. 95 auf dem Tomaros dargestellt.

\subsubsection{Verteilung des Gesangsmerkmals auf dem Tomaros}

\section{Gesamtverteilung}

Von den 150 auf Gesangsmerkmale hin untersuchten Individuen des Tomarosgebirges entsprechen 19 (12,67\%) der Art St. clavatus (Index 1). Der Art St. rubicundus (Index 12) werden 34 (22,67\%) der Individuen zugeordnet. Individuen die in ihren Gesängen der Art St. clavatus ähnlich sind, aber dieser nicht voll entsprechen, werden in Hybridindex 2; 3 und 4 eingeteilt. Dem Hybridindex 2 werden 20 (13,33\%), Index 3 - 27 (18,00\%) und Index 4 werden 12 (8,00\%) Tiere zugeordnet. Wie bereits erwähnt, sind sehr wenige Individuen mit völlig intermediären Gesängen (Index 5-8) auf dem Tomaros zu finden. Jedem dieser Hybridindices können nur zwischen $6(4,00 \%)$ und 2 (1,33\%) Individuen zugeordnet werden. Ebenfalls sehr wenige Individuen werden den Hybridindices 9 bis 11 zugeordnet. Diese Individuen zeigen in ihrem Verhalten Ähnlichkeiten zu St. rubicundus, entsprechen dieser aber nicht völlig. Jedem dieser Indices werden 5 (3,33\%) bis 11 (7,33\%) Individuen zugeteilt. 


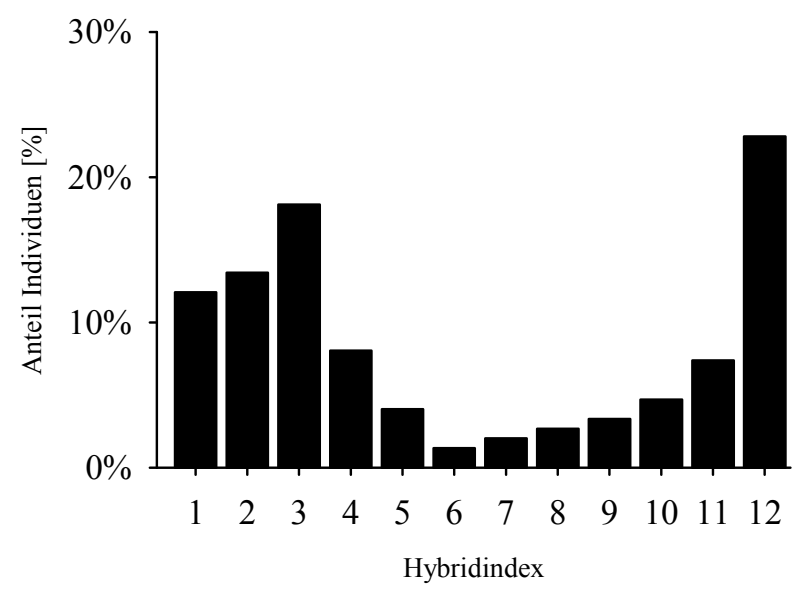

Abb. 94 Gesamtverteilung der Hybridindices (Gesangsmerkmal) auf dem Tomaros

\section{Detaillierte Beschreibung}

Nach dem gleichen Schema wie bei der Betrachtung der Merkmalsanteile der morphologischen Merkmale wird der durchschnittliche Merkmalsanteil des St. rubicundus-Merkmals der Individuen an jedem der betreffenden Standorte bestimmt. Von den insgesamt 45 Fundorten, an denen die Tiere zur Gesangsanalyse gesammelt wurden, sind 38 auf dem Tomaros zu finden. Sieben dieser Fundorte befinden sich auf dem Nord- und 31 auf dem Süd-Tomaros. Diese Fundorte und deren GPS-Kennungen werden in Abb. 95 und Abb. 96 dargestellt. 


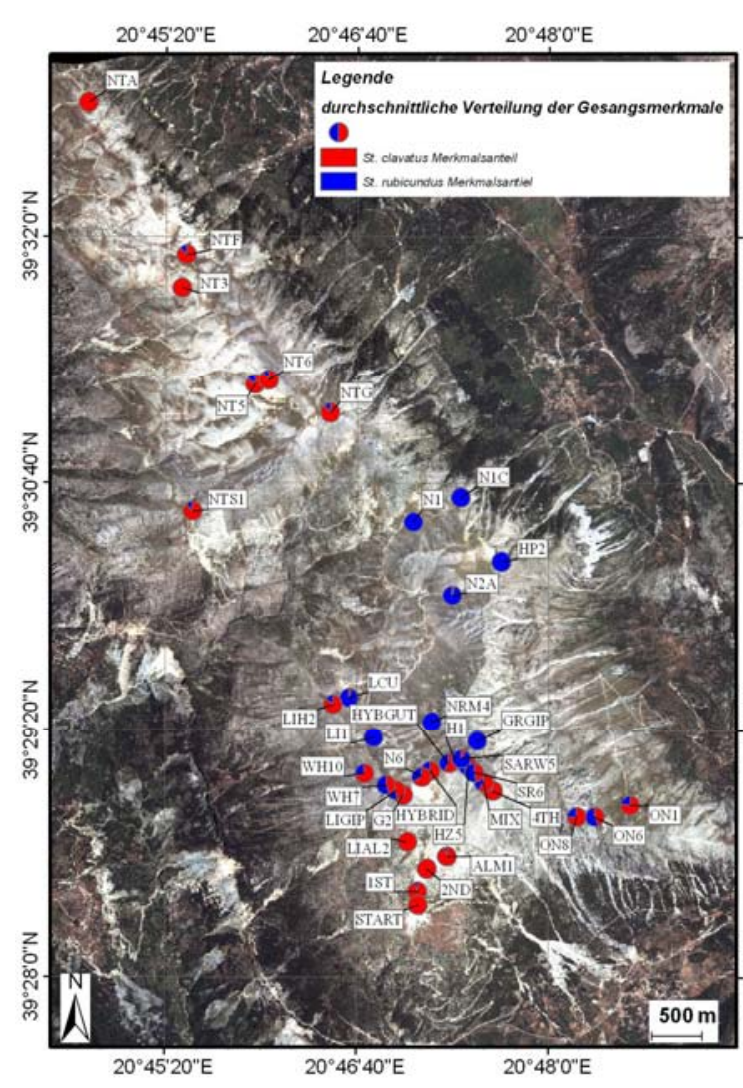

Abb. 95 Verteilung der durchschnittlichen Merkmalsanteile [\%] auf dem Tomaros

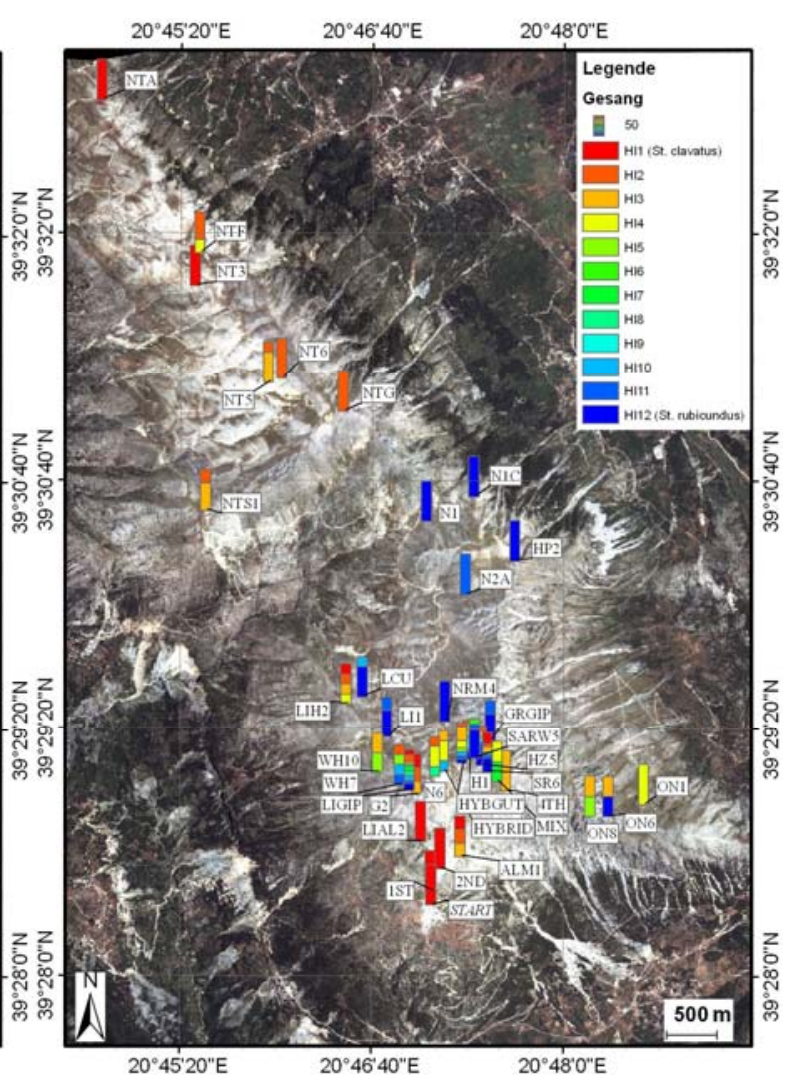

Abb. 96 Anteil der Individuen mit entsprechenden Hybridindices für jeden Fundort des Tomaros

Die Individuen der nördlichsten drei Fundorte des Nord-Tomaros (NTA, NTF; NT3) haben einen durchschnittlichen St. rubicundus-Merkmalsanteil von 6,15\%, variieren aber von Fundort zu Fundort zwischen 0\% und 12,30 \%. Somit zeigen diese Tiere einen Balzgesang der große Ähnlichkeiten zur Art St. clavatus aufweist.

Südlich dieser Fundorte befinden sich weitere vier GPS-Punkte (NT5, NT6, NTG, NTS1) von denen Individuen stammen, deren Verhalten aufgezeichnet wurde. An diesen Fundorten wurde ein durchschnittlicher Merkmalsanteil von 12,33\% ermittelt. Somit zeigen die Individuen einige Unterschiede zum Gesang der Art St. clavatus. Die mittleren Merkmalsanteile der Individuen an den einzelnen Fundorten variierten in diesem Gebiet zwischen $10,37 \%$ und $14,89 \%$.

Für die Betrachtung des Tomaros von Nord nach Süd wurden vier Fundorte ausgewählt, die sich im nord-östlichen Teil des Südtomaros befinden. Die Individuen in diesem Gebiet haben einen durchschnittlichen Merkmalsanteil von 99,11\% und entsprechen somit im Gesangsverhalten nahezu vollständig der Art St. rubicundus. 
Ausgehend von diesen Fundorten in südlicher Richtung sind Individuen von weiteren vier Fundorten (LCU, LI1, NRM4, GRGIP) auf ihren Gesang hin untersucht worden. Die Fundorte liegen in einer Reihe in Ost-West-Ausdehnung etwas nördlich vom Sattel des Südtomaros. Insgesamt ist ein durchschnittlicher Merkmalsanteil von 98,71\% gemessen worden. Somit zeigen diese Tiere ein Balzverhalten, das in sehr großen Teilen der Art St. rubicundus entspricht. Von Fundort zu Fundort variieren die Merkmalsanteile der Individuen zwischen 95,84 \% (LCU) und 100\% (Li1 und NRM4).

Am besten untersucht ist das Gebiet im Bereich des Sattels des Südtomaros. Hier wurde an insgesamt 13 Fundorten das Balzverhalten der Männchen aufgezeichnet. Diese 92 Männchen haben zusammen einen durchschnittlichen Merkmalsanteil von 47,39\% $(\sigma=39,00)$. Aber die durchschnittlichen Merkmalsanteile der Individuen an den einzelnen Fundorten können durchaus zwischen 7,00\% (G2) und 95,84\% (4TH) variieren. Die Betrachtung der Merkmalsanteile an den einzelnen Fundorten zeigt aber, dass die Männchen an acht Fundorten eine enorme Variabilität im Verhalten aufweisen. Man findet dort Individuen mit intermediären Gesangsmustern aber auch mit Gesängen, welche den Elternarten St. clavatus und St. rubicundus entsprechen. Östlich dieser Zone mit einer großen Merkmalsvariabilität im Verhalten sind an drei Standorten (ON1, ON6, ON8) im südlichen Teil des Osthanges das Verhalten der Männchen aufgezeichnet worden. Zusammen haben die fünf Individuen dieser Fundorte einen Merkmalsanteil von 37,55\%. Die Merkmalsanteile der Individuen an den einzelnen Fundorten können aber durchaus zwischen 22,56\% bei ON1 und $56,34 \%$ bei ON6 variieren.

Auf dem Südhang des Südtomaros wurde das Verhalten von Individuen von fünf Fundorten (1st, Start, 2nd, ALM1, LiAL2) untersucht. Ein durchschnittlicher Merkmalsanteil der Individuen dieser Standorte von 3,99\% ist errechnet worden. Die Merkmalsanteile der Individuen der einzelnen Punkte variieren aber durchaus zwischen 0\% und 6,47\%. Somit entsprechen die Individuen von diesen Standorten zum größten Teil den Individuen der Art St. clavatus.

\section{Zusammenfassung}

Auf den Nord-Tomaros zeigen die Individuen eines Fundortes ausschließlich Gesänge die große Ähnlichkeit zur Art St. clavatus aufweisen. Individuen von Fundorten aus den nörd- 
lichen Teilen des Nord-Tomaros zeigen eine größere Ähnlichkeit zu St. clavatus, als die untersuchten Individuen aus dem südlichen Teil.

Die Individuen zeigen im nördlichen Teil des Südtomaros ausschließlich Gesangsmuster, die der Art St. rubicundus entsprechen. Im südlichen Teil dieses Berges zeigen die von dort entnommenen Männchen einen Gesang, der der Art St. clavatus entspricht. Zwischen diesen beiden Gebieten findet man eine langgezogene Zone, in der die Individuen sowohl intermediären, als auch reinen, d. h. der Arten St. clavatus und St. rubicundus entsprechenden, Gesang zeigen.

\subsubsection{Interpolierte Verteilung der Gesangsmerkmale auf dem Tomaros}

Nach dem gleichen Prinzip, aber mit weitaus weniger Messpunkten (Fundorte mit durchschnittlicher Merkmalsverteilung der Individuen) ist eine Interpolation der Merkmalsanteile des Gesangs auf dem Tomaros durchgeführt worden. Ziel dieser Untersuchung ist es, ein zusammenhängendes Bild der Merkmalsverteilung sowie die Detektion möglicher Kontaktzonen herzustellen.

Anders als bei der Interpolation der morphologischen Merkmale, bei der drei Kontaktzonen deutlich wurden, ist bei der Interpolation des Verhaltens ausschließlich eine Kontaktzone zu erkennen. Dies liegt vor allem daran, dass das Verhalten bei weit aus weniger Individuen analysiert werden konnte als es bei den morphologischen Merkmale möglich war.

Auf dem Nord-Tomaros wurden bei den 15 Individuen ausschließlich St. clavatus ähnliche Gesangsmuster gefunden. Im nördlichen Teil dieses Gebietes ist der interpolierte Merkmalsanteil niedriger als im südlichen. Also sind die Individuen im nördlichen Teil des Nord-Tomaros im Verhalten der Art St. clavatus ähnlicher als die Tiere im südlichen Teil desselben Berges.

Der Südtomaros lässt sich durch die interpolierte Merkmalsverteilung in drei Teilgebiete gliedern. Im nördlichen Teil sind interpolierte Rasteroberflächen entstanden, die Hinweise darauf geben, dass sich in diesem Gebiet ausschließlich Individuen befinden, die Verhalten zeigen, welches der Art St. rubicundus entspricht (Merkmalsanteil 100\%) oder dieser Art sehr ähnlich ist (Merkmalsanteil von $90 \%$ bis 100\%). Wie auch bei den morphologischen Merkmalen erstreckt sich hier ein Gebiet, in dem die interpolierten Merkmalsanteile der 
Rasterzellen zwischen 30\% und 70\% liegen. Diese Kontaktzone erstreckt sich von der Nord-West-Kante des Südtomaros über den Sattel bis hin zum südlichen Teil des Osthanges. In diesem Gebiet zeigen die Individuen oftmals intermediäre Gesangsmuster. Auf dem Südhang des Südtomaros wurde eine Rasteroberfläche errechnet, die aus Zellen besteht, die Merkmalsanteile um 0\%, aber auch Zellen, die interpolierte Merkmalsanteile zwischen 2,5 und 10\%, aufweisen. Somit sind hier Individuen zu finden, die im Verhalten sehr große Ähnlichkeit zu St. clavatus aufweisen.
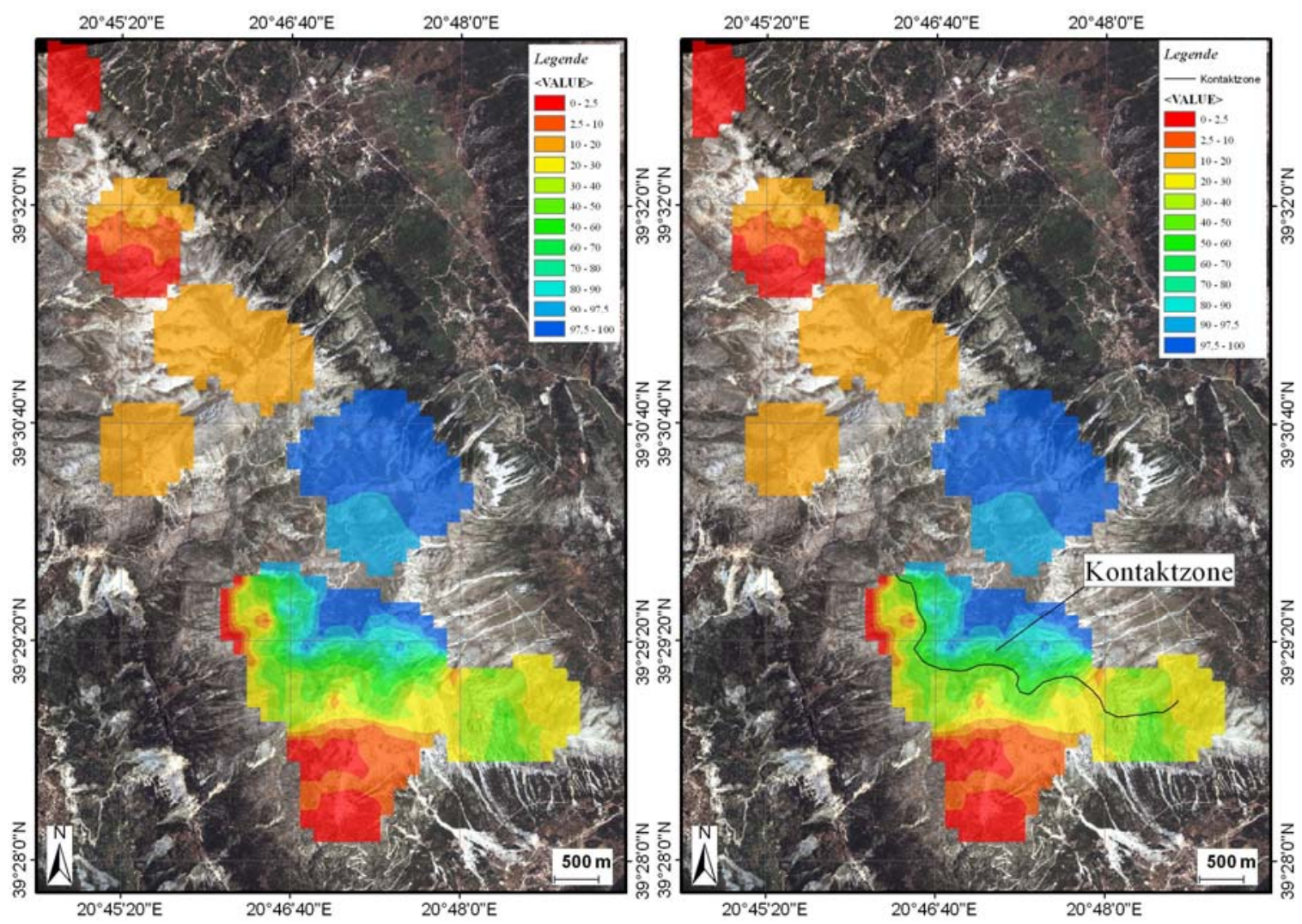

Abb. 97 Rasterstruktur der interpolierten Merkmalsverteilung (rechts) und interpolierte Merkmalsverteilung nach Glättung (bilineare Interpolation) der Gesangsmerkmale (links).

\subsubsection{Kline des Gesangsmerkmals}

Für die Betrachtung der Merkmalskline des Verhaltens wurden ausschließlich Tiere von den 30 Fundorten des Südtomaros herangezogen, da nur hier eine entsprechende Kontaktzone (Kontaktzone I) bestimmt werden konnte. Von jedem dieser Standorte wurde die kürzeste Distanz zur zuvor bestimmten Kontaktzone auf den Südtomaros berechnet. Werden nun die durchschnittlichen Merkmalsanteile der Individuen an den Fundorten gegen die kürzeste Entfernung aufgetragen, entsteht eine Kurve mit sigmoidem Verlauf (Abb. 98). 
Ausgehend vom Zentrum der Kontaktzone findet man bis zu einer Entfernung von $64 \mathrm{~m}$ in nördlicher Richtung Fundorte an denen die Individuen durchschnittliche Merkmalsanteile von 40\% bis 60\% aufweisen (Abb. 98 links). Im Abstand von $124 \mathrm{~m}$ bzw. $134 \mathrm{~m}$ sind auf dieser Seite der Kontaktzone weitere Fundorte mit Merkmalsanteilen zwischen $60 \%$ und $80 \% \mathrm{zu}$ finden. Ab einer Entfernung von $265 \mathrm{~m}$ findet man ausschließlich Fundorte an denen die Merkmalsanteile der Individuen zwischen 96\% und 100\% variieren. Somit ähneln die Individuen dieser Fundorte (GPS-Punkte) im Verhalten der Art St. rubicundus sehr oder entsprechen dieser.

Die Merkmalsverteilung auf der südlichen Seite der Hybridzone (Abb. 98 rechts) setzt sich folgendermaßen zusammen: Fundorte an denen den Individuen durchschnittliche Merkmalsanteile zwischen 40\% und 60\% zugeordnet werden, sind bis zu einem Abstand von $97 \mathrm{~m}$ in südlicher Richtung vom Zentrum der Kontaktzone zu finden. In diesem Bereich findet man besonders häufig Individuen mit intermediären Gesangsmustern. Bis zu einem Abstand von 175 m vom Zentrum der Kontaktzone sind ebenfalls Fundorte mit Merkmalsanteilen zwischen $40 \%$ und 10\% zu finden. Die dort vorkommenden Individuen zeigen bereits mehrheitlich Gesangsmuster die der Art St. clavatus ähneln. Ab einer Entfernung von $200 \mathrm{~m}$ zum Zentrum der Kontaktzone verändern sich die durchschnittlichen Merkmalsanteile der Individuen an den Fundorten nur noch leicht und variieren zwischen 0\% und 8\%, entsprechen somit der Art St. clavatus oder ähneln dieser.

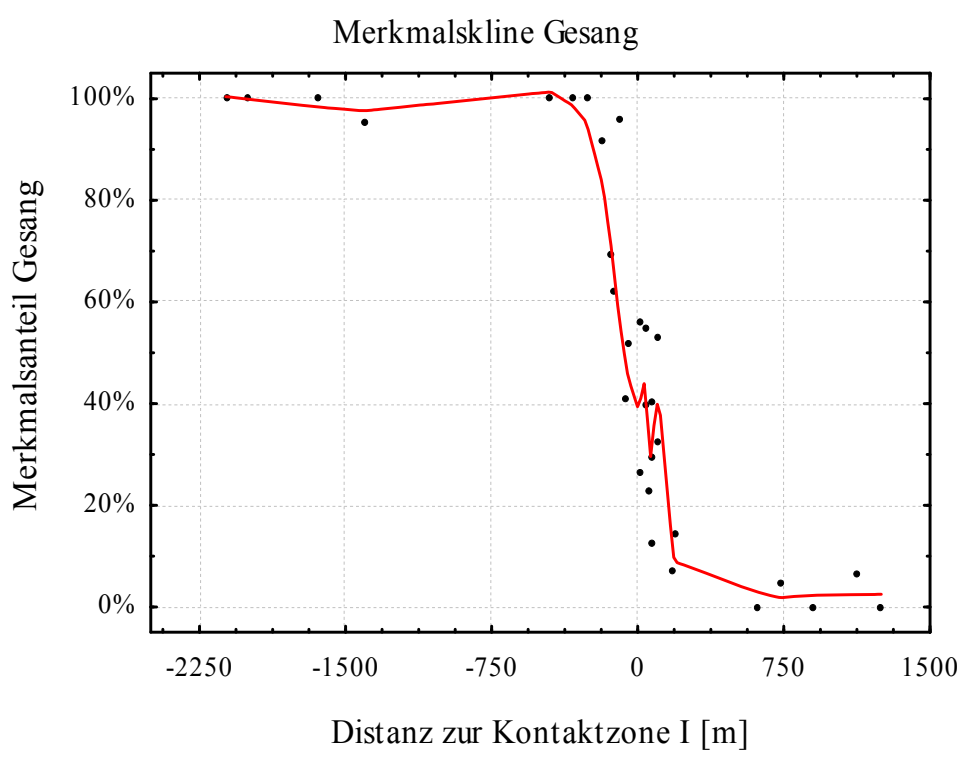

Abb. 98 Gesangskline im Bereich der Kontaktzone I. Lowess-Glättung $(-)$ h=0,25 


\section{Zusammenfassung}

Die Zone, in der die durchschnittlichen Merkmalsanteile an den Fundorten zwischen 40\% und $60 \%$ liegen, ist ca. $150 \mathrm{~m}$ breit. Flankiert wird diese Zone von Gebieten, in denen sich die Merkmalsanteile an den einzelnen Fundorten mit zunehmender Distanz zum Zentrum der Kontaktzone entweder der Art St. clavatus in südlicher Richtung oder der Art St. rubicundus und nördlicher Richtung annähern. Ab einem Abstand von 175 m bzw. 265 m stabilisieren sich die Merkmalsanteile an den Fundorten auf dem Niveau der Arten St. clavatus (Süden) und St. rubicundus (Norden).

\subsection{Vergleich der verschiedenen Merkmalskline}

In den vorangegangenen Kapiteln wurden die Kline der Flügel-, Antennen- und Gesangsmerkmale genau beschrieben. Auf Grundlage dieser Werte werden nun die Merkmalskline miteinander verglichen. Anschließend werden durch Hauptkomponentenanalyse alle Merkmale zusammen in einem Raster dargestellt.

\subsubsection{Kontaktzone I}

Flügel-, Antennen- und Gesangsmerkmale sind im Bereich der Kontaktzone I untersucht worden (Abb. 99). Hierbei zeigen alle drei Merkmalskline einen sigmoiden Kurvenverlauf der in großen Teilen fast identisch ist. Deckungsgleich sind vor allem die Kurven des Gesangs und Flügelmerkmals in unmittelbarer Nähe der Kontaktzone. Hingegen sind die Kline des Antennenmerkmals in diesem Bereich um ca. 30 Meter in Richtung Norden (St. rubicundus-Seite) verschoben. Nach ca. $350 \mathrm{~m}$ zu beiden Seiten der Kontaktzone stabilisieren sich die durchschnittlichen Anteile aller drei Merkmale. Auf der Seite der Art St. rubicundus (Nord bis Ost) pegeln sich die Kline der Gesangs- und Flügelmerkmale bei Werten um $100 \%$ ein und entsprechen so den Merkmalen der St. rubicundusReferenzpopulationen. Anders als die Gesangs- und Flügelmerkmale sind die Antennenmerkmale in diesem Bereich der Kline sehr variabel und schwanken stark zwischen $83 \%$ und 98\%. Durchschnittlich liegen die Merkmalsanteile in diesem Bereich bei 91,32\% $(\sigma=10,6)$. Dadurch haben diese Individuen Antennen, die im Vergleich zu den St. rubicundus-Referenzpopulationen schon leichte Veränderungen aufweisen.

Bei der Betrachtung der Merkmalskline auf der Seite der Art St. clavatus (Süd bis West) pegeln sich die Merkmalskline des Gesangs- und Antennenmerkmals bei Werten um 0\% 
ein. Die Individuen aus diesen Gebieten haben also Antennen- und Gesangsmerkmale die der Art St. clavatus entsprechen. Etwas anders sieht es aber bei den Flügelmerkmalen aus, die in diesem Bereich einen durchschnittlichen Merkmalsanteil von 16,28\% $(\sigma=8,99)$ aufweisen. Diese Individuen zeigen im Vergleich zu St. clavatus eine enorme flügelmorphologische Veränderung und ähneln dieser Art nur noch leicht.

Die Kline der Gesangsmerkmale erreichen auf beiden Seiten der Kontaktzone Werte, die den Referenzpopulationen entsprechen. Hingegen ist dies bei den Klinen des Flügel- und Antennenmerkmals nur auf jeweils einer der beiden Seiten zu beobachten. Bei den Flügelmerkmalen zeigen Individuen auf der Seite der Art St. clavatus eine Veränderung hin zu St. rubicundus. Ähnliches ist bei den Antennen auf der entgegengesetzten Seite, der Kontaktzone (St. rubicundus-Seite) zu beobachten. Hier zeigen die Antennen der Individuen leichte Verdickungen die eine leichte Veränderung hin zur Art St. clavatus bedeuten. Unterzieht man die Flügel- und Antennenmerkmale einer Regressionsanalyse, so unterscheiden sich beide Merkmalskline in ihrer Steilheit und sind ebenfalls signifikant verschoben (Abb. 99).

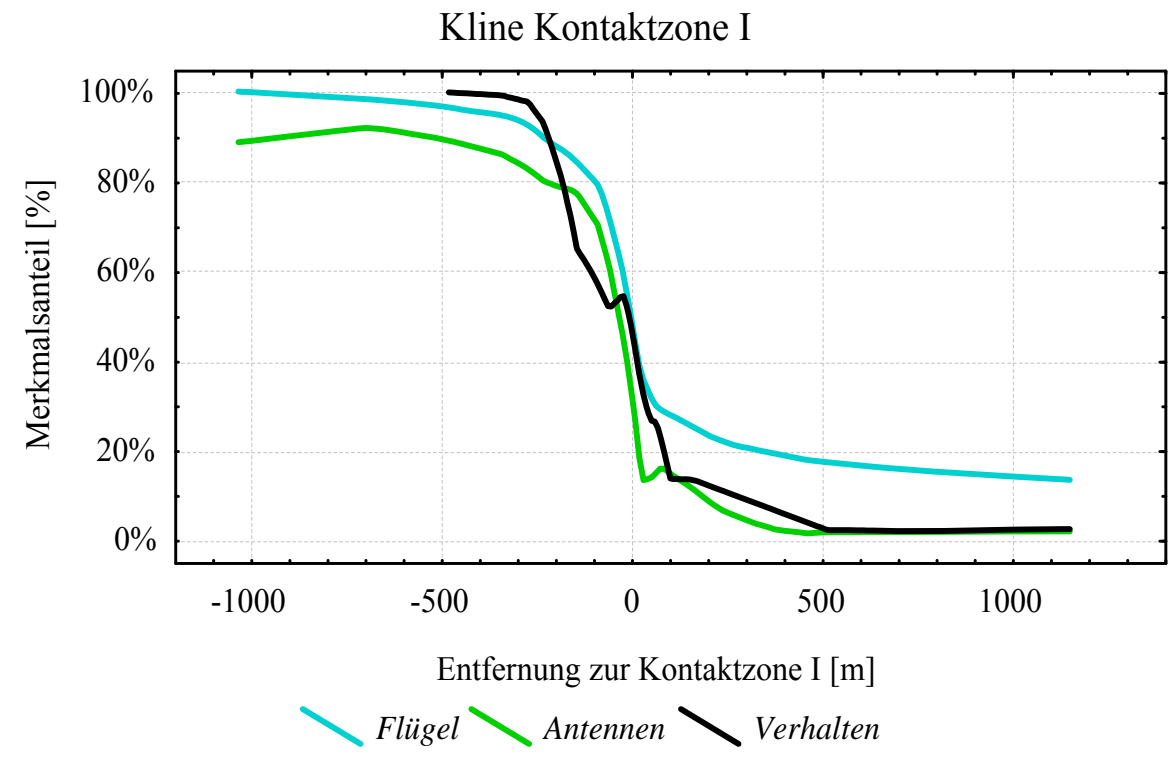

Abb. 99 Kline ausgehend von Kontaktzone I. Lowess-Glättung (-) h=0,25

\subsubsection{Kontaktzone II und III}

Im Gegensatz zu den Klinen der Kontaktzone I ist der Verlauf der Kline im Bereich der Kontaktzone II und III nicht sigmoid. Die Gesangskline konnten hier aufgrund zu weniger Daten nicht dargestellt werden. Ungeachtet dessen gibt es einige Gemeinsamkeiten zwi- 
schen den Klinen aller drei Kontaktzonen. Die Merkmalskline sind um ca. 10 bis 15 Prozentpunke verschoben. Dies heißt, dass der durchschnittliche Merkmalsanteil des Antennenmerkmals an den einzelnen Fundorten um 10 bis 15 Prozentpunkte kleiner ist. Diese Verschiebung wird ebenfalls durch die Regressionsanalyse bestätigt, bei der signifikante Unterschiede in der Weite und Steilheit berechnet worden sind.

Betrachtet man Kontaktzone III, bei der vor allem die Merkmale auf der Seite der Art St. rubicundus fehlen, fällt ebenfalls der Unterschied zwischen Flügel- und Antennenmerkmal auf. Aber im Gegensatz zu den anderen beiden Kontaktzonen ist dieser mit Merkmalsunterschieden von fünf Prozentpunkten nicht besonders groß. Des Weiteren bleibt dieser Unterschied über die Länge der Kline konstant. Die Individuen in diesem Gebiet haben somit durchschnittliche Vorderflügelmerkmalsanteile von ca. 5\% und Antennenmerkmale um 0\%. Dieser Zustand ist bereits ab einem Abstand von ca. $200 \mathrm{~m}$ zur Kontaktzone III zu beobachten.

Merkmalskline Kontaktzone II

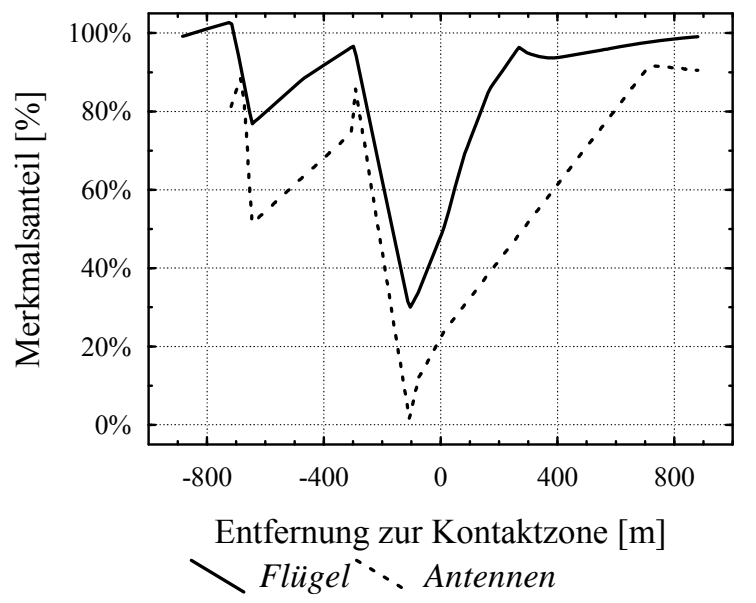

Merkmalskline Kontaktzone III

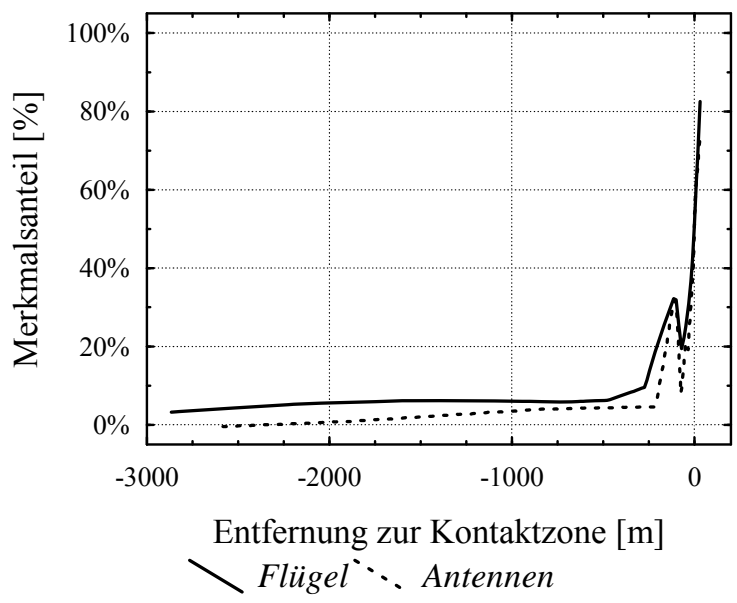

Abb. 100 Kline ausgehend von Kontaktzone II (links) und III (rechts). Lowess-Glättung (-) h=0,25

\section{Zusammenfassung}

Zusammenfassend sind bis zu einem Abstand von $100 \mathrm{~m}$ bis $350 \mathrm{~m}$ von beiden Seiten zu den Kontaktzonen viele Individuen mit intermediären Merkmalen zu finden. Die Breite dieser Zone unterscheidet sich von Kontaktzone zu Kontaktzone. Bei Kontaktzone II und III ist diese Zone $200 \mathrm{~m}$ bis $300 \mathrm{~m}$ ( $100 \mathrm{~m}$ bis $150 \mathrm{~m}$ zu beiden Seiten) breit und $700 \mathrm{~m}$ bei Kontaktzone I.

St. rubicundus des Tomaros, die nicht in der Nähe der Kontaktzonen zu finden sind, besitzen eine Vorderflügelmorphologie, die im Großen und Ganzen der griechischen St. rubi- 
cundus-Vergleichpopulation entspricht. Hingegen sind deren Antennen etwas breiter als die der Referenzpopulationen. Das Verhalten dieser Individuen entspricht wiederum den Referenzpopulationen. Bei St. clavatus des Tomaros ist dies genau umgekehrt, die Antennenmorphologie entspricht der Referenzgruppe, aber die Flügelmorphologie dieser Individuen zeigt jedoch bereits leichte Unterschiede zur Referenzgruppe, und zwar in Richtung St. rubicundus.

\subsection{Laborkreuzungen}

Da die intermediären Formen auf dem Tomaros hinsichtlich des Kreuzungstyps nicht zu bestimmen sind, wurden zusätzlich zur Untersuchung der Wildfänge auch Laborkreuzungen zwischen Individuen der beiden Arten St. clavatus und St. rubicundus vorgenommen. Dadurch sollten erste Hinweise zur Klärung der Verhältnisse auf dem Tomaros gewonnen werden. Es wurden Individuen der beiden Arten miteinander verpaart, aber auch deren Nachkommen untereinander gekreuzt oder mit einer der Elternarten rückgekreuzt. Von den Nachkommen dieser Kreuzungen wurden von insgesamt 112 (55^^; 57우) Individuen die Vorderflügel- und von 61 (38 §ో; 23q) die Antennen morphometrisch vermessen. Der Balzgesang wurde von 45 Männchen aufgenommen. Bei den Kreuzungstypen steht in allen Fällen an erster Stelle das Weibchen, an zweiter das Männchen

Im Folgenden werden die Vorderflügel- und Antennenmorphologie und das Verhalten der Individuen aus den Kreuzungsexperimenten anhand von Merkmalsanteilen und Hybridindices beschrieben. Begonnen wird mit den F1 Hybriden, und später werden auch die Individuen der F2 Generation näher betrachtet.

\subsubsection{F1 Hybride}

Für die Untersuchung der Individuen der F1-Generation aus der Kreuzung wurden Individuen der allopatrischen Populationen der Arten St. clavatus und St. rubicundus untereinander sowie Individuen der parapatrischen Populationen dieser Arten auf dem Tomaros miteinander verpaart. Es wurden Kreuzungen zwischen St. clavatus Weibchen mit St. rubicundus Männchen und deren reziprokem Kreuzungstyp durchgeführt. Des Weiteren wurden St. clavatus Weibchen vom Tomaros mit Männchen der Art St. rubicundus desselben Berges miteinander verpaart. Schließlich wurde auch ein Weibchen der allopatrischen St. clavatus Population des Xerovouni mit einem Männchen der parapatrischen St. rubicundus Population des Tomaros miteinander verpaart. 
Tab. 27 Durchschnittliche Größe des Vorderflügels und Femurlänge. Legende: Tomaros (T)

\begin{tabular}{|c|c|c|c|c|c|c|c|c|c|}
\hline F1 Hybride & \multicolumn{3}{|c|}{ Flügel } & \multicolumn{3}{|c|}{ Antennen } & \multicolumn{3}{|c|}{ Verhalten } \\
\hline Kreuzungstyp & Anz. & MA & $\boldsymbol{\sigma}$ & Anz. & MA & $\boldsymbol{\sigma}$ & Anz. & MA & $\boldsymbol{\sigma}$ \\
\hline St. clavatus x St. rubicundus & 44 & $51,72 \%$ & 14,03 & 21 & $61,96 \%$ & 27,28 & 10 & $45,98 \%$ & 10,13 \\
\hline St. rubicundus x St. clavatus & 6 & $56,46 \%$ & 6,26 & 4 & $58,06 \%$ & 13,01 & 4 & $69,37 \%$ & 5,39 \\
\hline St. clavatus $(\mathrm{T}) \mathrm{x}$ St. rubicundus $(\mathrm{T})$ & 4 & $68,82 \%$ & 17,03 & 4 & $49,68 \%$ & 16,94 & 1 & $31,82 \%$ & \\
\hline St. clavatus x St. rubicundus $(\mathrm{T})$ & 9 & $53,19 \%$ & 8,19 & 2 & $46,67 \%$ & 13,44 & 4 & $42,33 \%$ & 18,67 \\
\hline
\end{tabular}

Probleme gab es vor allem bei der Kreuzung zwischen St. rubicundus Weibchen und St. clavatus Männchen. Aus dem Großteil der Eier, die aus dieser Verpaarung entstammten, schlüpften keine oder nur sehr wenige Larven. Erst zum Ende der vorliegenden Arbeit ist es gelungen, einige wenige F1-Hybriden dieses Typs zu ziehen. Bei den anderen Kreuzungstypen sind solche Probleme nicht von Bedeutung gewesen.

\subsubsection{Flügelmorphologie}

Bei der morphometrischen Untersuchung der Flügel wurden die Individuen aus der ersten Filialgeneration (F1) Hybridindices von 5 bis 9 zugeordnet Der Großteil der Individuen der F1 Generation weist also sehr intermediäre Merkmale auf. Eine Ausnahme bildet ein Individuum aus der Kreuzung zwischen St. clavatus $q$ und St. rubicundus $\hat{\sigma}$ aus den parapatrischen Populationen des Tomaros, das mit Hybridindex 11 flügelmorphologisch der Art St. rubicundus sehr ähnlich ist. Die durchschnittlichen Merkmalsanteile der F1-Kreuzungen liegen zwischen 51,72\% (St. clavatus x St. rubicundus) und 68,82\% (St. clavatus (T) x St. rubicundus $(\mathrm{T})$.

\subsubsection{Antennenmorphologie}

Bei der Antennenvermessung waren den F1-Hybriden Indices von 4 bis 10 zuzuordnen; sie zeigten somit eine etwas größere Varianz als bei der Flügelmorphologie. Trotzdem besaBen alle Nachkommen in der F1-Generation intermediäre Antennenmerkmale. Nur einzelne Individuen hatten aufgrund ihrer Antennenmorphologie eine etwas größere Ähnlichkeit zur Art St. rubicundus (Index 10). Bildet man die durchschnittlichen Merkmalsanteile der Nachkommen aus den verschiedenen Kreuzungstypen der F1-Generation, so erhält man durchschnittliche Merkmalsanteile zwischen 46,67\% (St. clavatus x St. rubicundus (T) und 61,96\% (St. clavatus x St. rubicundus). Individuen der F1-Generation besitzen Antennen, die morphologisch intermediäre Merkmale aufweisen. 


$$
\mathbf{F 1}=\text { St. clavatus } \uparrow \mathbf{x} \text { St .rubicundus } \curvearrowright
$$

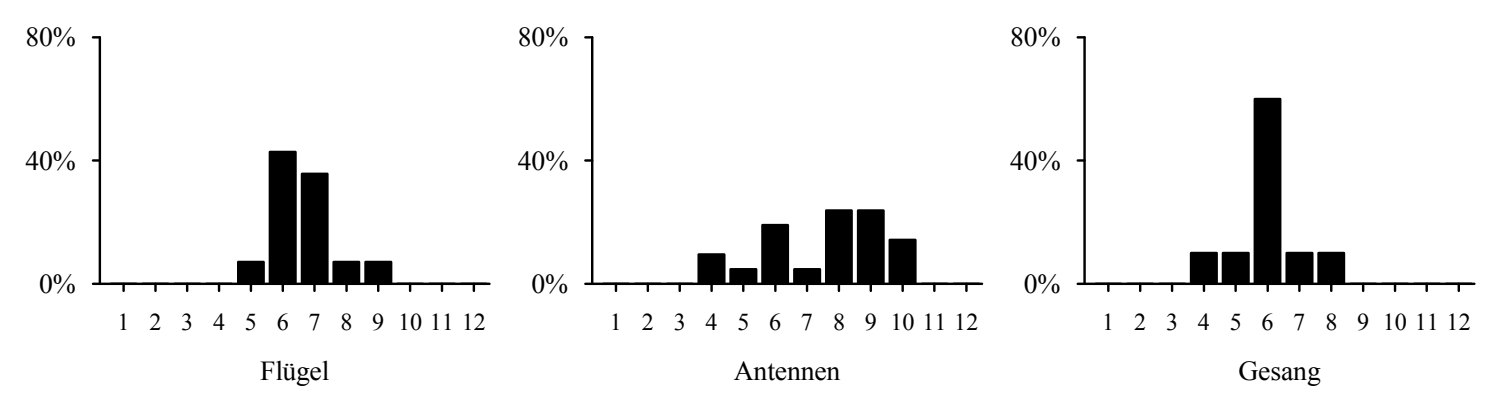

$\mathbf{F 1}=$ St. rubicundus $\stackrel{+}{\mathbf{x} \text { St. clavatus } \curvearrowright}$
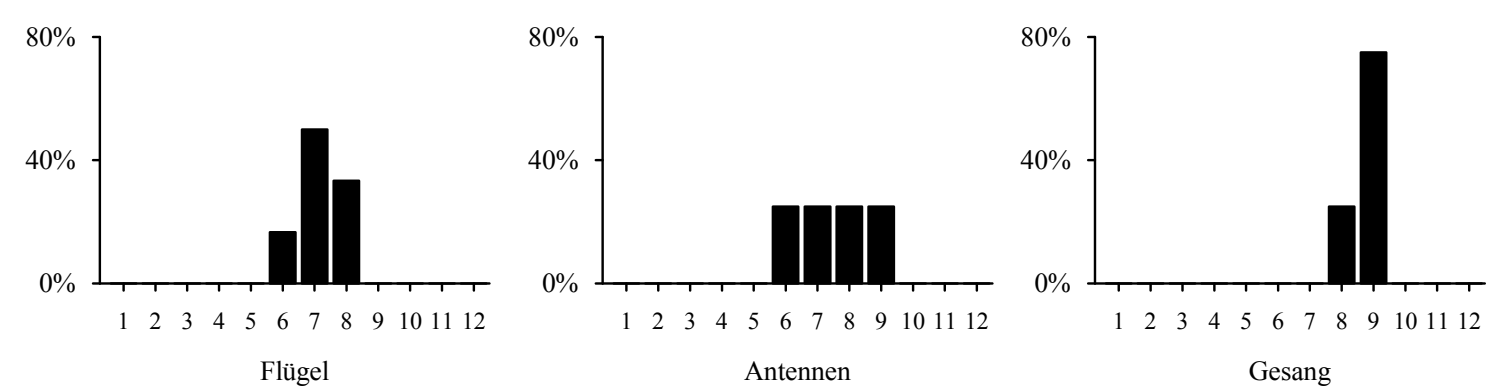

\section{$\mathbf{F} 1=$ St. clavatus $\bigcirc \mathbf{x}$ St. rubicundus $($ Tomaros) $\curvearrowright$}
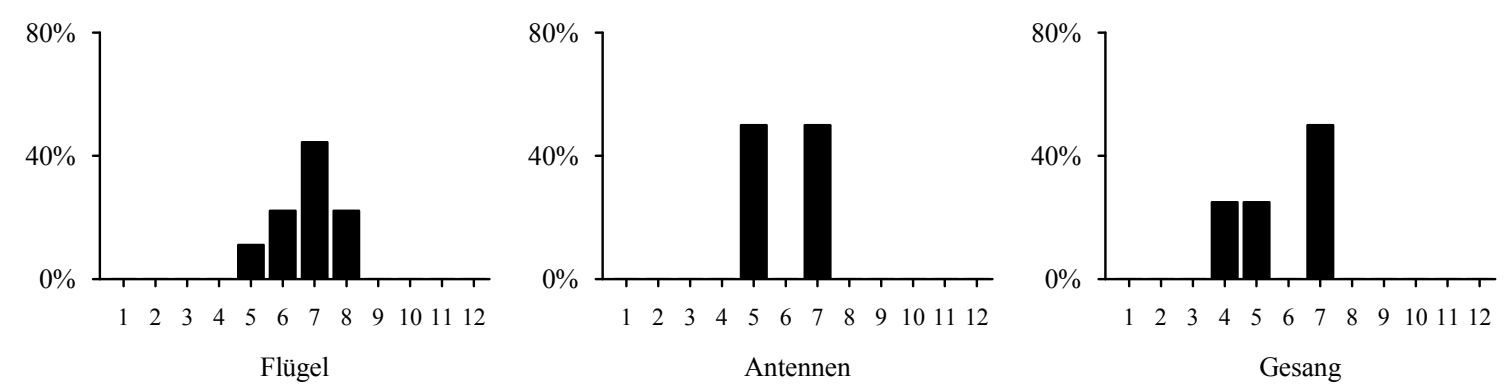

F1 = St. clavatus (Tomaros) $\uparrow \mathbf{x}$ St. rubicundus (Tomaros) $\curvearrowright$
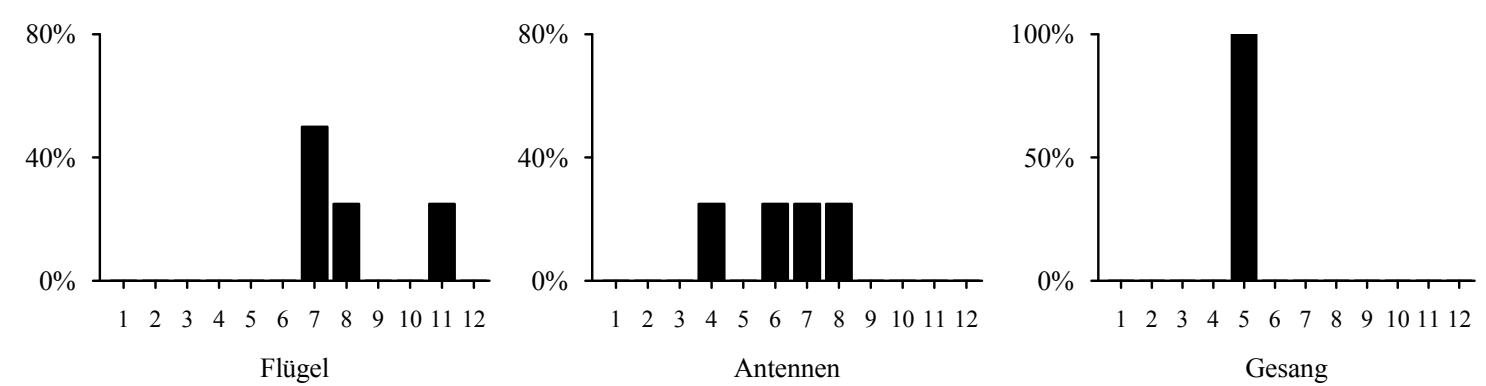

Abb. 101 Hybridindices der Flügel-, Antennen- und Gesangsmerkmale der F1 Generation aus Laborkreuzungen x-Achsen: 1 entspricht der Art St. clavatus (Xerovouni) und 12 der Art St. rubicundus. y-Achsen: Prozentualer Anteil an Individuen.

\subsubsection{Balzgesang}

Der Balzgesang der F1-Männchen ist äußerst variabel. Es werden meist in Phase II und III Mischformen der entsprechenden Elterngesänge gezeigt. Die Phasen III werden oftmals ohne jegliche Unterschiede zu den Elternarten dargeboten (Abb. 102 und Abb. 103). 


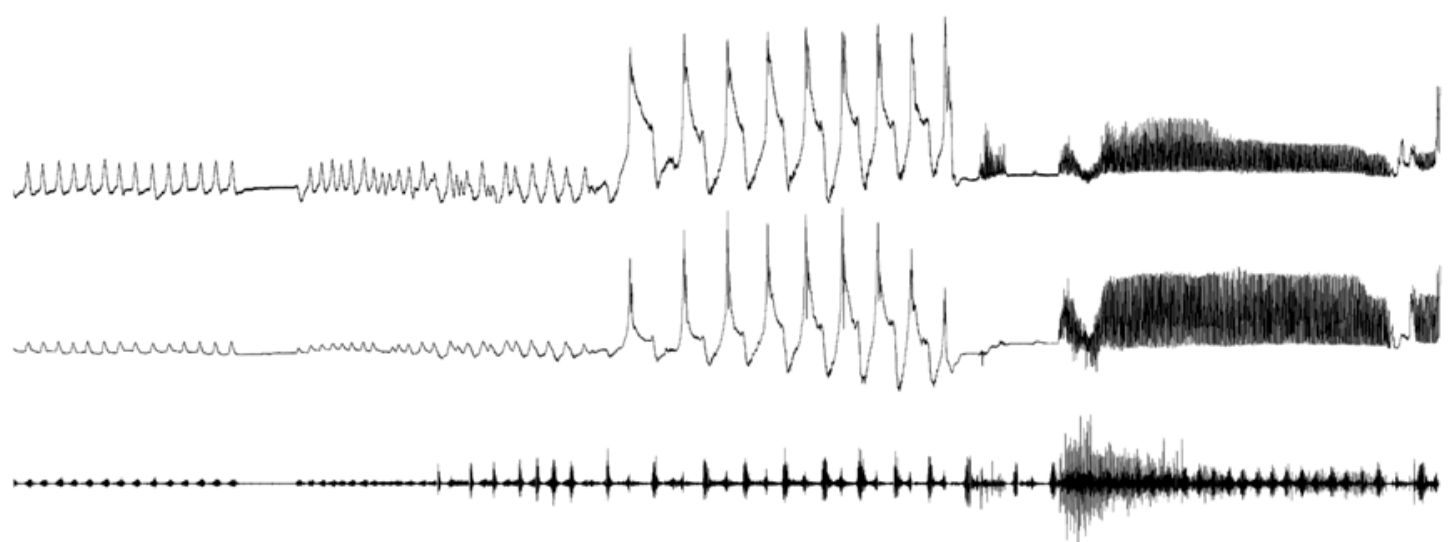

Abb. 102 Gesang eines F1-Hybrid Männchens (St. clavatus x St. rubicundus) Bewegungsspur des linken (oben) und des rechten (Mitte) Hinterbeins sowie die dazugehörende Tonspur (unten).

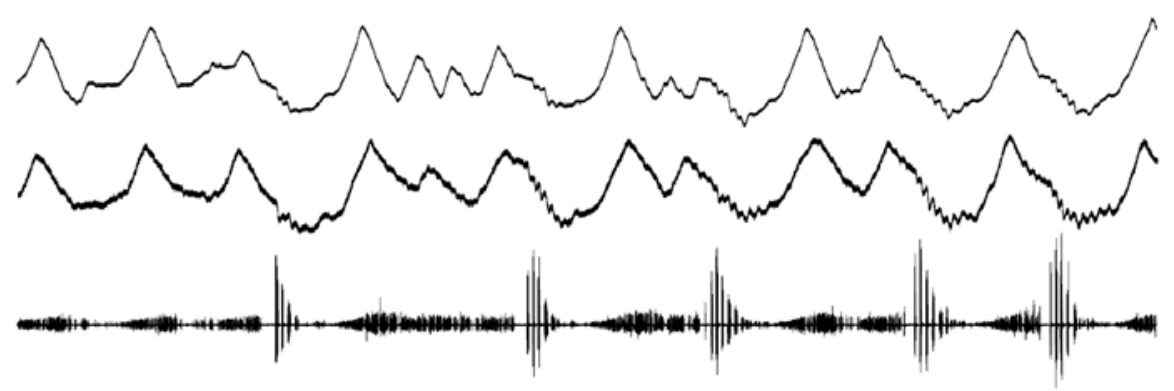

Abb. 103 Gesangsphase eines F1-Hybrid Männchens (St. clavatus x St. rubicundus) Bewegungsspur des linken (oben) und des rechten (Mitte) Hinterbeins sowie die dazugehörende Tonspur (unten).

Von den F1-Männchen, deren Verhalten aufgezeichnet wurde, zeigten alle Individuen insgesamt einen intermediären Gesang. Dabei fiel auf, dass vor allem die zentralen Phasen (Phase III) beider Elternarten präsentiert worden sind. Unmittelbar vor dieser zentralen Phase wurden oftmals statt eines Einstreuens von Flügelschlägen, dies ist vor allem bei St. rubicundus zu beobachten, laute stufige Abwärtsbewegungen gezeigt (Abb. 103). Die F1Hybriden wurden auf Grundlage ihres Verhaltens in Hybridindices von 4 bis 8 eingeteilt. Durchschnittlich wurde ein Merkmalsanteil von 31,82\% (St. clavatus (T) x St. rubicundus (T)) bis 69,37\% (St. rubicundus x St. clavatus) ermittelt. Diese Daten zeigen, dass im Vergleich zur Flügelmorphologie das Verhalten der Tiere variabler ist. Es spielen vor allem maternale Effekte beim Verhalten eine Rolle. So liegen die durchschnittlichen Merkmalsanteile der F1-Hybriden aus den verschiedenen Kreuzungstypen meist leicht auf der Seite des weiblichen Elternteils. 


\subsubsection{F2-Hybride}

$\mathrm{Zu}$ den F2-Hybriden gehören in erster Linie Individuenkreuzungen aus zwei F1-Hybriden oder Kreuzungen, die aus Rückkreuzungen zwischen F1-Hybriden und den Elternarten hervorgehen. Es wurden für diese Kreuzungen ausschließlich F1-Hybriden des Kreuzungstyps St. clavatus Weibchen und St. rubicundus Männchen verwendet. Grund dafür ist der schlechte Zuchterfolg des reziproken Kreuzungstyps (siehe Kapitel 3.6.1). Es wurden Nachkommen von folgenden Kreuzungstypen gezogen und näher untersucht: Durch Kreuzung zweier F1-Hybride (St. clavatus x St. rubicundus) wurden zudem Hybriden der zweiten Generation erzeugt. F2-Hybriden aus Rückkreuzungen entstanden durch folgende Kreuzungstypen: St. rubicundus x F1 (St. clavatus x St. rubicundus) und St. clavatus x F1 (St. clavatus x St. rubicundus). Von den beiden letzteren Kreuzungstypen wurden zusätzlich auch die reziproken Kreuzungstypen herangezogen. Es gab dabei bis auf den Kreuzungstyp: St. rubicundus x F1 (St. clavatus x St. rubicundus) keine größeren Zuchtprobleme. Aber aus dem eben genannten Kreuzungstyp sind sehr wenige Individuen geschlüpft und dementsprechend auch wenige groß gezogen worden.

Im Folgenden werden nun die Ergebnisse aus den morphometrischen Vermessungen der Antennen und der Flügel, sowie aus der Verhaltensanalyse der F2-Hybriden genauer beschrieben. Dies geschieht wiederum auf Grundlage der Hybridindices und Merkmalsanteile; diese Methode ist bereits bei der Untersuchung der Wildfänge etabliert und in Kapitel 2.5 näher beschrieben worden.

Tab. 28 Durchschnittliche Größe des Vorderflügels und Femurlänge. Legende St. clavatus (C), St. rubicundus (R) und F1 (St. clavatus x St. rubicundus).

\begin{tabular}{|c|c|c|c|c|c|c|c|c|c|}
\hline \multirow{2}{*}{$\begin{array}{c}\text { F2 Hybride } \\
\text { Kreuzungstyp } \\
\end{array}$} & \multicolumn{3}{|c|}{ Flügel } & \multicolumn{3}{|c|}{ Antennen } & \multicolumn{3}{|c|}{ Verhalten } \\
\hline & Anz. & MA & $\sigma$ & Anz. & MA & $\sigma$ & Anz. & MA & $\sigma$ \\
\hline $\mathrm{F} 1(\mathrm{C} \times \mathrm{R}) \times \mathrm{F} 1(\mathrm{C} \times \mathrm{R})$ & 8 & $55,80 \%$ & 22,24 & 4 & $58,95 \%$ & 15,17 & 2 & $60,13 \%$ & 23,89 \\
\hline St. rubicundus x F1(C x R) & 18 & $80,65 \%$ & 11,54 & 14 & $71,73 \%$ & 28,78 & 8 & $85,68 \%$ & 16,79 \\
\hline $\mathrm{F} 1(\mathrm{C} \times \mathrm{R}) \times \mathrm{St}$. rubicundus & 3 & $62,69 \%$ & 15,57 & 1 & $64,55 \%$ & - & 2 & $65,60 \%$ & 29,76 \\
\hline St. clavatus x F1(C x R) & 9 & $13,88 \%$ & 12,64 & 8 & $13,59 \%$ & 15,55 & 8 & $20,25 \%$ & 9,74 \\
\hline $\mathrm{F} 1(\mathrm{C} \times \mathrm{R}) \times \mathrm{St}$. clavatus & 11 & $23,51 \%$ & 18,94 & 10 & $38,21 \%$ & 23,15 & 6 & $14,62 \%$ & 6,95 \\
\hline
\end{tabular}

\subsubsection{Flügelmorphologie}

Die acht morphometrisch vermessenen Nachkommen der F1-Hybriden (Kreuzung F1 x F1) werden auf Grund ihrer Flügelmorphologie in Hybridindices von 5 bis 10 eingeteilt und haben somit intermediäre Flügelmerkmale (Abb. 104). Anders als ihre Eltern zeigen diese 
F2 $=$ F1 (St. clavatus x St .rubicundus) $\uparrow$ x F1 (St. clavatus x St. rubicundus) $\curvearrowright$
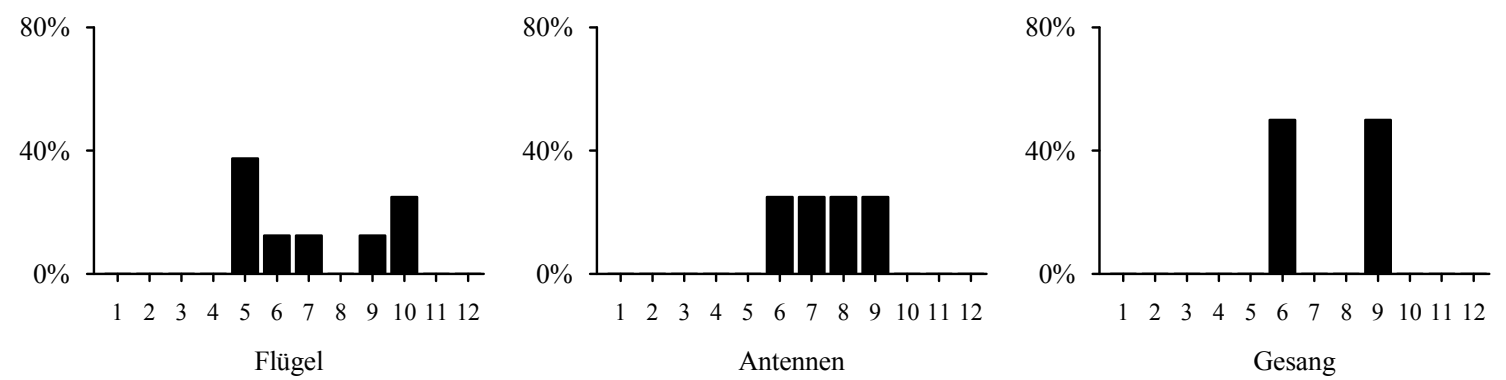

$\mathbf{R K}=$ St. rubicundus $\uparrow$ x F1 Hybrid (St. clavatus $\mathbf{x}$ St. rubicundus)
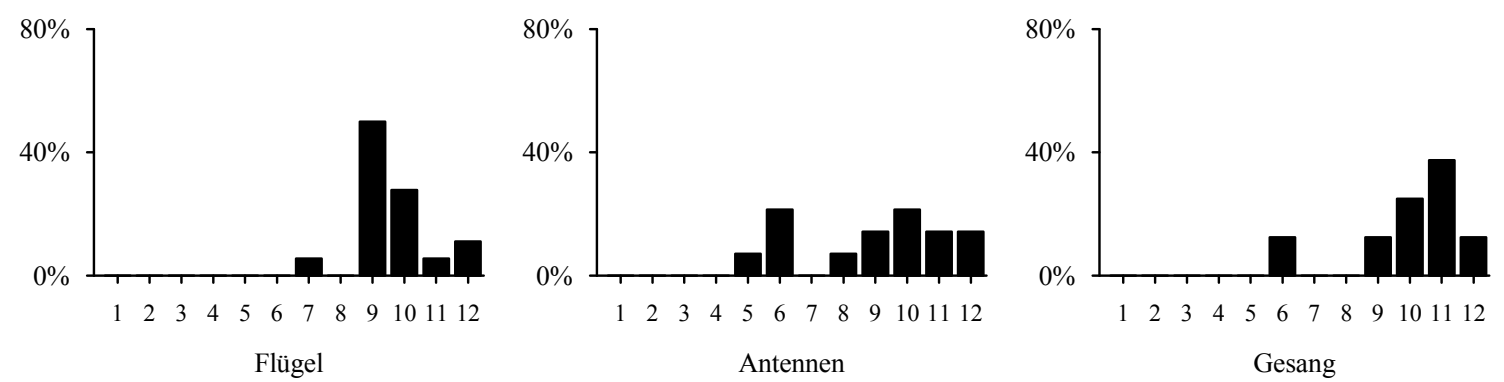

RK $=$ F1 Hybrid + (St.clavatus $\mathbf{x}$ St. rubicundus) $\mathbf{x}$ St. rubicundus $\hat{\jmath}$
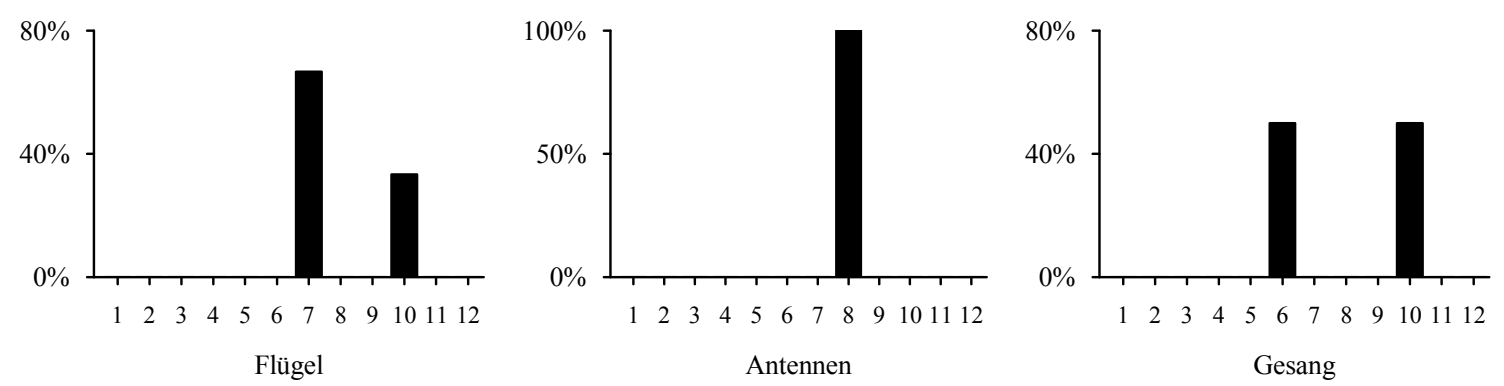

RK = St. clavatus $\uparrow$ x F1 Hybrid (St. clavatus x St. rubicundus) $\curvearrowright$
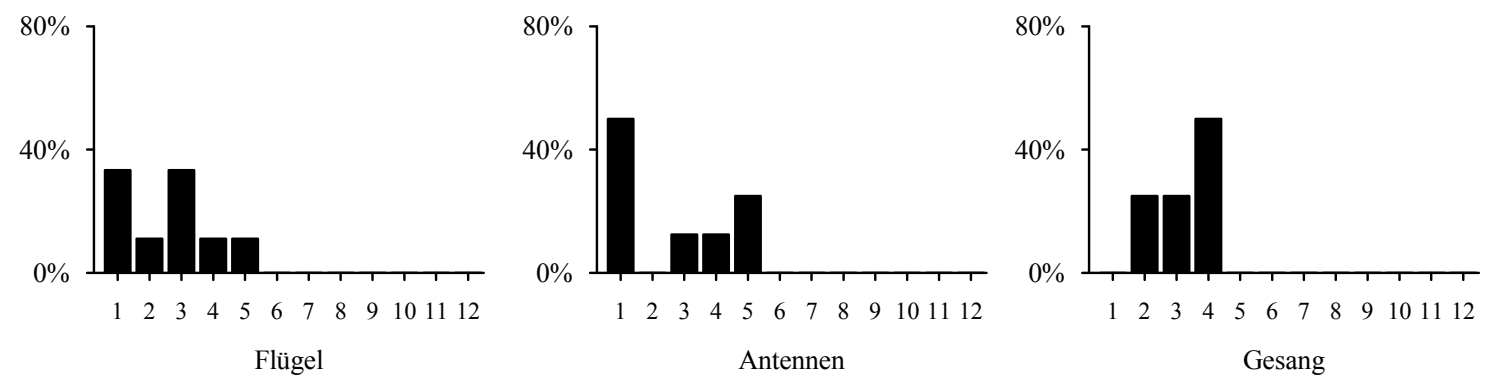

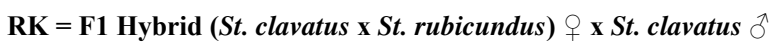
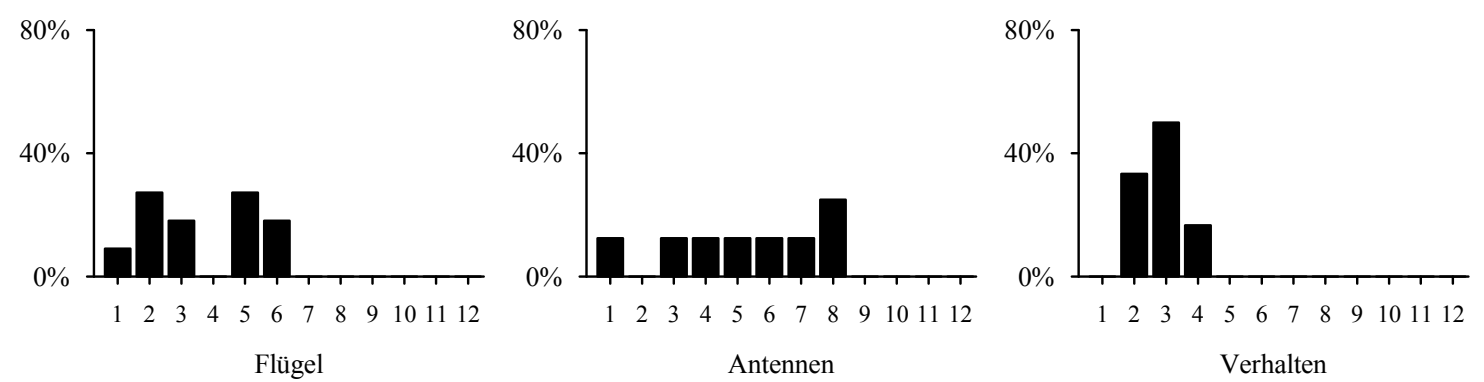

Abb. 104 Hybridindices der Flügel-, Antennen- und Gesangsmerkmale der F1 Generation aus Laborkreuzungen. x-Achsen: 1 entspricht der Art St. clavatus (Xerovouni) und 12 der Art St. rubicundus. y-Achsen: Prozentualer Anteil an Individuen 
Tiere aber eine größere Varianz. Der durchschnittliche Merkmalsanteil liegt auch bei den F2-Hybriden bei $55,80 \%(\sigma=22,24)$.

Bei den Rückkreuzungen unter Beteiligung der Art St. rubicundus zeigen die Individuen, die in Hybridindices von 7 bis 12 eingeteilt wurden, Merkmale im intermediären Bereich sowie Merkmale, die der Art St. rubicundus sehr ähnlich sind oder dieser sogar entsprechen. Der durchschnittliche Merkmalsanteil, aus der Rückkreuzung zwischen St. rubicundus x F1 (St. clavatus x St. rubicundus), liegt bei 80,65 \% $(\sigma=11,54)$ und somit nah an der Art St. rubicundus. Beim reziproken Kreuzungstyp wurde ein durchschnittlicher Merkmalsanteil von $62,69 \%(\sigma=15,57)$ errechnet. Daraus resultiert, dass diese Individuen hauptsächlich intermediäre Merkmale aufweisen.

Aus den Rückkreuzungen mit Beteiligung von St. clavatus sind die Individuen durch morphometrische Vermessung in Hybridindices von 1 bis 6 eingeteilt worden. Somit haben diese Individuen intermediäre Merkmale, aber auch Flügelmerkmale, die der Art St. clavatus sehr ähnlich sind oder dieser entsprechen. Es sind keine morphologischen Überlappungen zu den Rückkreuzungen mit Beteiligung von St. rubicundus gefunden worden. Die durchschnittlichen Merkmalsanteile der Individuen liegen beim Kreuzungstyp: St. clavatus x F1 (St. clavatus x St. rubicundus) bei 13,88\% $(\sigma=12,64)$ und somit haben diese Individuen große Gemeinsamkeiten der Art St. clavatus. Ähnliche Werte sind beim reziproken Kreuzungstyp mit einem durchschnittlichen Merkmalsanteil von 23,51\% $(\sigma=18,94)$ zu finden. Diese Tiere zeigen eine etwas geringere Ähnlichkeit zur Art St. clavatus als die Individuen des Kreuzungstyps: St. clavatus x F1 (St. clavatus x St. rubicundus).

\subsubsection{Antennenmorphologie}

Durch morphometrische Berechnung sind die Individuen der F2 Generation aus F1 Hybriden (St. clavatus x St. rubicundus) in Hybridindices von 6 bis 9 eingeteilt und liegen daher im intermediären Bereich. Ein durchschnittlicher Merkmalsanteil von 58,94\% $(\sigma=15,17)$ macht zusätzlich deutlich, dass die Antennen morphologisch auch leichte Tendenzen zu St. rubicundus aufweisen.

Rückkreuzungen zur Art St. rubicundus erhalten aufgrund ihrer Antennenmorphologie Hybridindices zwischen 5 und 12. Also sind, wie bei der Flügelmorphometrie, Individuen 
mit intermediären, aber auch Merkmalen die der Art St. rubicundus sehr ähnlich sind oder dieser entsprechen, zu finden. Dies spiegelt sich ebenfalls bei den Merkmalsanteilen wider. Die Individuen des Kreuzungstyps: St. rubicundus x F1 (St. clavatus x St. rubicundus) weisen einen durchschnittlichen Merkmalsanteil von $71,73 \%(\sigma=28,78)$ auf und zeigen so leichte Ähnlichkeiten zur Art St. rubicundus. Beim reziproken Kreuzungstyp liegt der durchschnittliche Merkmalsanteil bei $64,55 \%$ und somit ebenfalls etwas mehr auf der Seite der Art St. rubicundus.

Weiter wurden die Antennen der Individuen, die aus Rückkreuzungen mit St. clavatusBeteiligung stammen, vermessen. Kreuzt man St. clavatus mit F1-Hybriden (St. clavatus x St. rubicundus), so lassen sich die Antennen der Nachkommen in Hybridindices von 1 bis 8 einteilen. Morphologisch entstehen Individuen mit intermediärer Antennenmorphologie, sowie Tiere die bei den Antennen der Art St. clavatus sehr ähnlich sind oder dieser gar entsprechen (Index 1). Nachkommen aus dem Kreuzungstyp: St. clavatus x F1 Hybrid (St. clavatus x St. rubicundus) erhalten auf Grund ihrer Antennenmorphologie einen durchschnittlichen Merkmalsanteil von 13,88\% $(\sigma=15,55)$ und zeigen somit eine große Ähnlichkeit zur Art St. clavatus. Einen größeren durchschnittlichen Merkmalsanteil von 38,21\% $(\sigma=23,15)$ haben die Individuen des reziproken Kreuzungstyps (St. clavatus x F1 Hybrid [St. clavatus x St. rubicundus]). Dies deutet darauf hin, dass diese Individuen durchschnittlich mehr intermediäre Antennenmerkmale haben, als die des anderen Kreuzungstyps.

\subsubsection{Balzgesang}

Das Verhalten der Individuen der F2-Generation zeichnet sich durch eine hohe Variabilität aus und zeigt ähnlich wie in der F1-Generation vor allem in Phase I und II noch intermediäre Merkmalszüge (Abb. 105 bis Abb. 107). Es ist kein Individuum gefunden worden, das in Phase I Merkmale zeigt, die einer der Arten St. clavatus oder St. rubicundus entspricht. In der Phase II werden Phasen dargeboten, die mehr oder weniger einer der beiden Arten entsprechen. Oft findet man hier aber auch wieder Flügelschläge oder in gleicher Art und Weise präsentierte laute stufige Abwärtsbewegungen. Unter den Individuen der F2 Generation sind nur noch sehr wenige Individuen zu finden, die die beiden dritten Phasen der Arten St. clavatus und St. rubicundus präsentierten. Meist zeigen diese Tiere nur eine der Phasen III. 


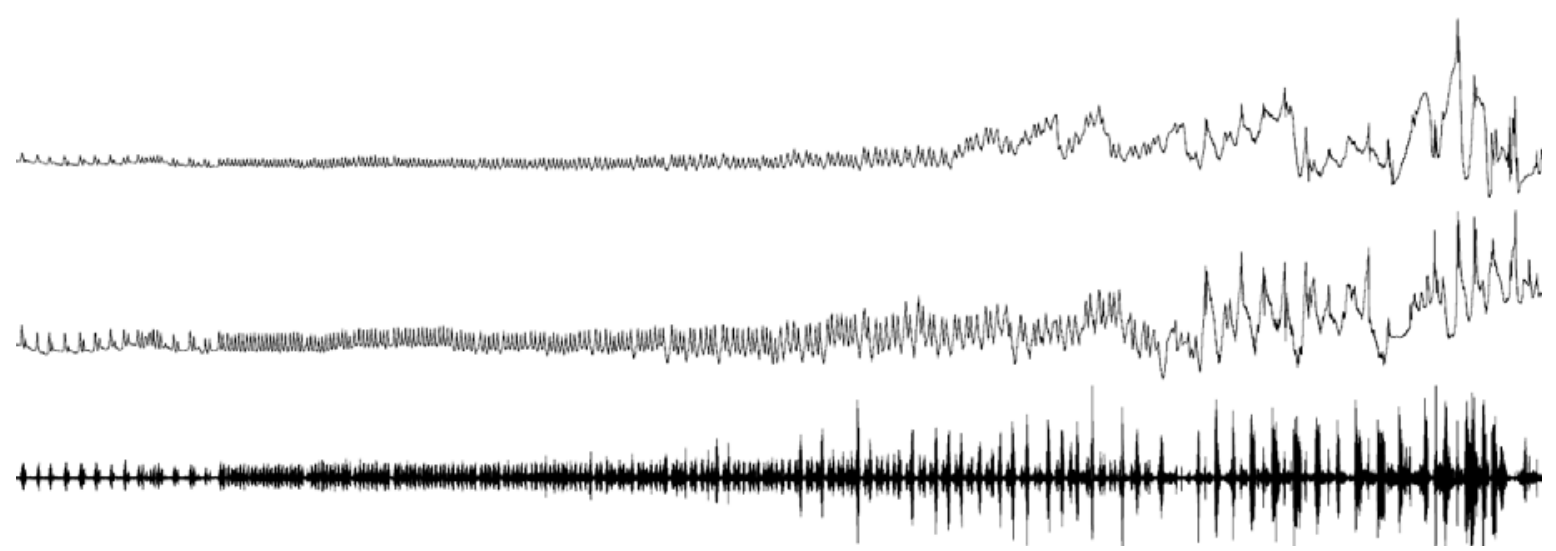

Abb. 105 Gesang der Laborkreuzungen (F1+F1). Bewegungsspur des linken (oben) und des rechten (Mitte) Hinterbeins sowie die dazugehörende Tonspur (unten).

Auf Grundlage der in Kapitel 3.4.5 beschriebenen systematischen Einordnung der Gesänge in Hybridindices werden die zwei Individuen aus der Kreuzung von F1-Weibchen und F1Männchen in die Hybridindices 6 und 9 eingeteilt. Das heißt, dass die beiden Individuen einen intermediären Balzgesang zeigen. Eines der Individuen zeigt aber im Gesang etwas mehr Tendenzen zur Art St. rubicundus. Der durchschnittliche Merkmalsanteil beider Tiere liegt bei $60,13 \%(\sigma=23,89)$ und somit zeigen diese Tiere intermediären Gesang mit leichten Tendenzen zum Gesang der Art St. rubicundus.

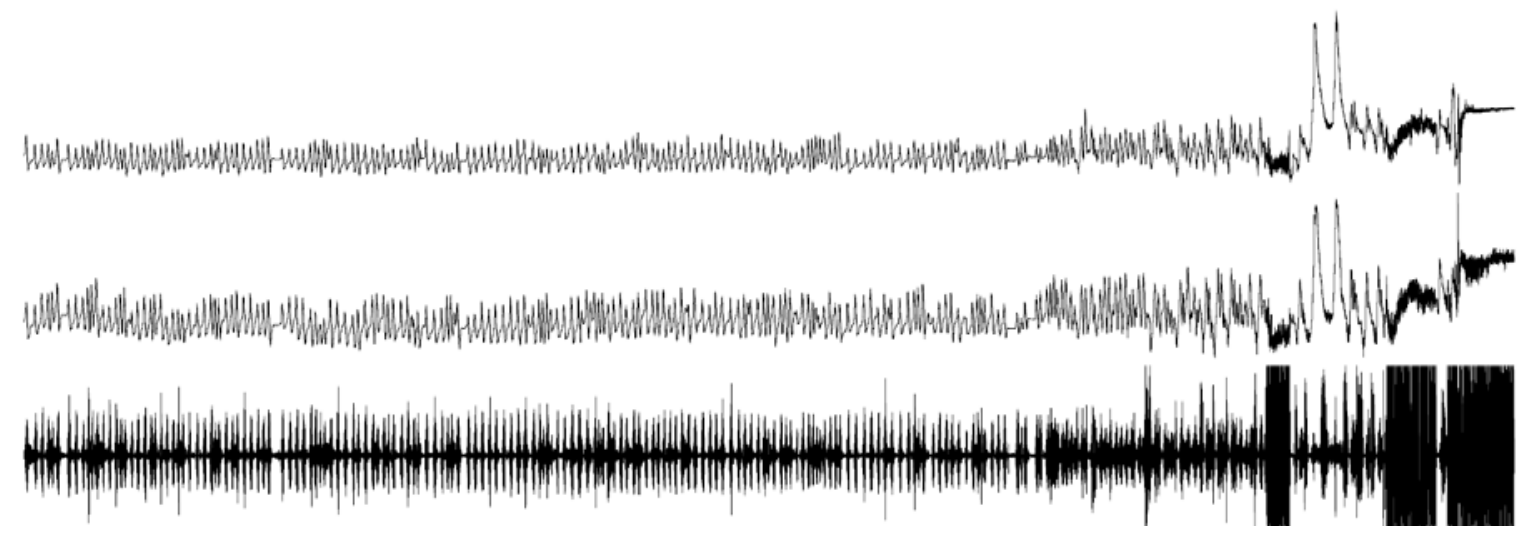

Abb. 106 Gesang der Laborkreuzungen St. rubicundus + F1. Bewegungsspur des linken (oben) und des rechten (Mitte) Hinterbeins sowie die dazugehörende Tonspur (unten).

Männliche Nachkommen aus den Rückkreuzungen zur Art St. rubicundus werden auf Grundlage ihrer Gesänge in Hybridindices von 6 bis 12 eingeordnet. Infolge dessen singen diese Männchen intermediär, ähnlich der Art St. rubicundus oder dieser entsprechend. Nachkommen aus Kreuzungen von St. rubicundus x F1 (St. clavatus x St. rubicundus) haben durchschnittlich einen Merkmalsanteil von 85,68 \% $(\sigma=16,76)$, deren Balz zeigt somit sehr große Gemeinsamkeiten zur Balz der St. rubicundus Männchen. Nachkommen des 
umgekehrten Kreuzungstyps hingegen haben einen Merkmalsanteil von 64,55\%. Da aber nur ein Individuum untersucht wurde, ist dieser Wert wenig aussagekräftig.

Rückkreuzungen zur Art St. clavatus werden in Hybridindices von 2 bis 4 eingeteilt. Bei diesen Tieren ist also die Variabilität im Gesang weitaus kleiner als bei den Rückkreuzungen mit St. rubicundus-Beteiligung. Im Gesang ähneln somit die Nachkommen dieser Rückkreuzungen den Gesängen der Art St. clavatus, entsprechen aber dieser nicht vollständig. Der durchschnittliche Merkmalsanteil im Gesang der Nachkommen aus der Rückkreuzung St. clavatus x F1 Hybrid (St. clavatus x St. rubicundus) liegt bei 20,25\% (6,95) und der reziproke Kreuzungstyp zeigt mit durchschnittlich 14,62\% $(9,74)$ einen ähnlichen Wert.

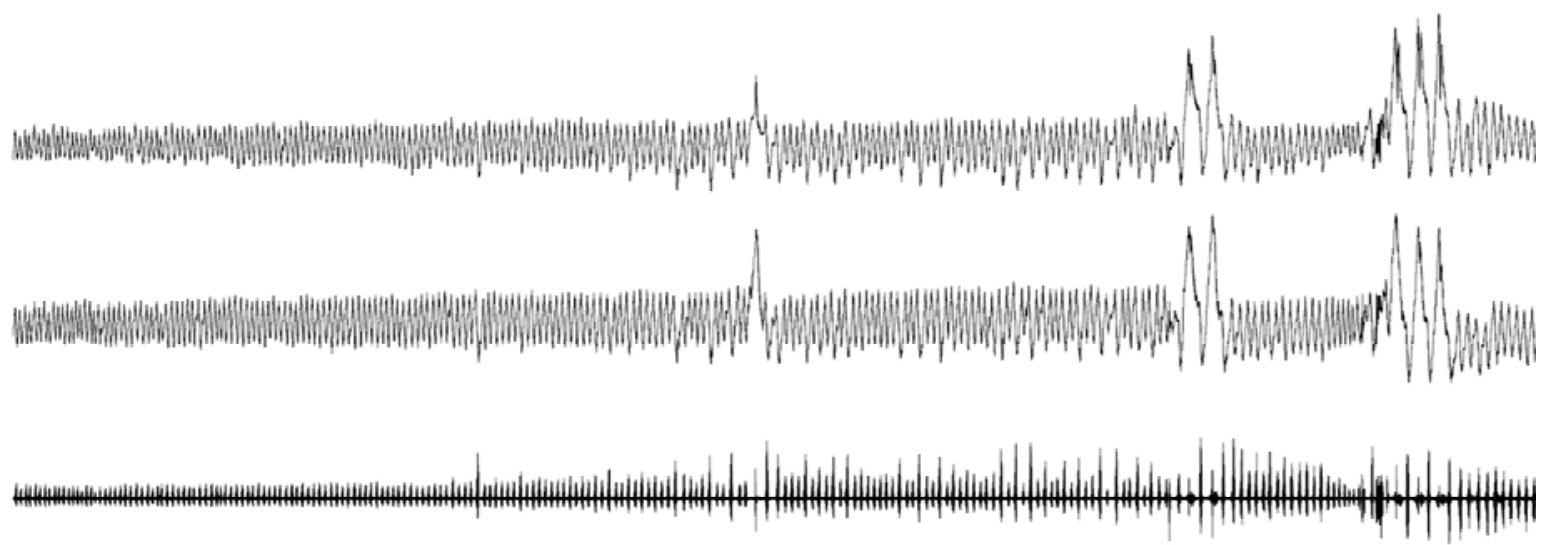

Abb. 107 Gesang der Laborkreuzungen F1(St. clavatus x St. rubicundus) x St. clavatus. Bewegungsspur des linken (oben) und des rechten (Mitte) Hinterbeins sowie die dazugehörende Tonspur (unten). 


\section{Diskussion}

Unmittelbares Ziel der vorliegenden Dissertation war es, die beiden Feldheuschreckenarten Stenobothrus clavatus und St. rubicundus sowie deren mögliche Hybriden sowohl hinsichtlich ihrer Morphologie und ihres Verhaltens als auch ihres Verbreitungsgebietes zu charakterisieren. Im Vordergrund stand dabei die Frage, ob diese Untersuchungen etwas zur Entstehung von Hybridzonen, Etablierung von Evolutionsmechanismen und generell zu Fragen der Artbildung in isolierten Populationen beitragen können. Es geht also generell darum, zu prüfen, inwieweit sich die Hybridzone am Tomaros für eine Untersuchung von evolutionsbiologischen Fragestellungen eignet.

Im Folgenden werden die Ergebnisse dieser Arbeit diskutiert. Zunächst werden neben der Besprechung der Methoden die beiden Arten St. rubicundus und St. clavatus sowie die Hybridzone zwischen beiden faunistisch eingeordnet. Im Anschluss werden die Vorkommnisse auf dem Tomaros unter evolutionsbiologischen Gesichtspunkten diskutiert.

\subsection{Methodische Betrachtung}

Für die Charakterisierung der Populationen wurden Flügel und Antennen morphometrisch vermessen, sowie die Balzgesänge der Männchen aufgezeichnet. Somit konnten die Individuen der untersuchten Populationen verglichen und auf Grundlage ihrer Merkmale eingeordnet werden.

\subsubsection{Flügelmorphometrie}

Die Vorderflügel der Heuschrecken dienten in der vorliegenden Arbeit als eines von zwei morphologischen Merkmalen. Sie wurden sowohl mit traditionellen morphometrischen als auch mit geometrisch morphologischen Methoden vermessen. Die Oberfläche des Flügels sowie die Femurlänge wurden im Zuge der klassischen Morphometrie untersucht. Durch 13 geometrische Messpunkte, gesetzt an charakteristischen Stellen der Vorderflügel, wurde die Flügelstruktur morphometrisch erfasst. Mit beiden Methoden konnten Unterschiede auf Art- und Populationsebene detektiert werden, wobei sich die Vermessung des Vorderflügels mit geometrischen Messpunkten als die effektivere und genauere von beiden Metho- 
den erwies. Anders als bei der klassischen Morphometrie muss bei der geometrischen Morphometrie nur ein Organ untersucht werden, weil bei dieser Methode Größenparameter durch Prokrustesanalyse normiert werden können (Bookstein 1991). Diese Methode wurde erfolgreich zur Untersuchung von Fliegen, Käfern und Bienen angewandt (De la Riva et al. 2001, Garnier et al. 2005, Bischoff et al. 2009). Bei Heuschrecken wurde sie bisher noch nie verwendet, hat sich nun aber auch hier bewährt. Untersuchungen von anderen Arten der Gattung Stenobothrus zeigten im Zuge der vorliegenden Arbeit, dass es durchaus möglich ist, Arten dieser Gattung nur aufgrund ihrer Flügelmorphologie zu unterscheiden und einzuordnen. Daraus kann man schließen, dass die Speziation einiger Arten der Gattung Stenobothrus auch mit der Veränderung der Flügelmorphologie einher geht.

Flügelmorphometrische Vergleiche können in erster Linie zur Zuordnung von Individuen zu bestimmten Arten verwendet werden (Bischoff et al. 2009). Darüber hinaus ist es möglich, intermediäre Zwischenformen, z. B. in Hybridzonen, zu charakterisieren. Schlussfolgerungen über den Verwandtschaftsgrad bzw. die phylogenetische Einordnung von Arten der Gattung Stenobothrus aufgrund der Flügelmorphometrie können mit dieser Methode jedoch nicht gezogen werden.

\subsubsection{Antennenmorphometrie}

Antennenmorphometrische Vermessungen bei Heuschrecken sind eher selten, boten sich aber für die Untersuchung des Artunterschiedes zwischen St. rubicundus und St. clavatus an. Hier wurde die Breite der distalen Antennalsegmente gegen die der proximalen gemessen. Anders als bei der flügelmorphometrischen Vermessung wurde diese Methode ausschließlich zur Charakterisierung der Arten St. rubicundus und St. clavatus sowie deren Zwischenformen verwendet, für welche diese sich gut eignete. Dies ist vor allem dadurch begründet, dass St. clavatus zu den wenigen Heuschreckenarten gehört, die verbreiterte Antennenspitzen aufweisen.

Wie schon bei der flügelmorphometrischen Vermessung, gelang es mit dieser Methode die Individuen des Tomaros exakt einzuordnen. Bei den vermessenen Antennen der Individuen in der Hybridzone konnten intermediäre Merkmale, aber auch einige, die einer der Elternarten entsprechen, detektiert werden. Ähnlich wie die Flügel für St. rubicundus, scheinen verbreiterte Antennen den Individuen der Art St. clavatus bei der Balz als optisches Signal zu dienen. Vermutet wird, dass von den Antennen möglicherweise auch Duftstoffe (z. B. 
Kohlenwasserstoffe) abgegeben werden könnten, die unter Geschlechtspartnern oder Rivalen zur intra- und interartlichen Kommunikation dienen (Lockey 1988; Neems und Butlin 1994, Tregenza et al. 2000a, b; Buckley et a. 2003).

\subsubsection{Verhaltensdetektion}

Für die Verhaltensuntersuchung wurde der Balzgesang der Männchen der Arten St. rubicundus und St. clavatus aufgezeichnet. Hierzu wurden die Beinbewegungen aber auch die erzeugten Laute aufgenommen und mittels acht Merkmalen charakterisiert. Anhand dieser Merkmale ist es gelungen, die Arten zu differenzieren, sowie die Individuen des Tomaros einzuordnen. Diese Methode eignet sich gut zur Untersuchung von Artunterschieden bei Orthopteren, da ihre Gesänge artspezifisch und sind und eine geringe innerartliche Variation zeigen (Jacobs 1953, Faber 1953, Elsner 1974a, Ragge \& Reynolds 1998). Da die Weibchen in hohem Maße selektiv sind und sich in der Regel nur paaren, wenn das Männchen einen für das Weibchen attraktiven Werbegesang vorträgt, spielt der Gesang für die Evolution der Heuschrecken eine wichtige Rolle (Helversen 1972, Helversen \& Helversen 1994). Den Weibchen kommt also eine entscheidende Rolle bei der Artbildung zu (Dobzhansky 1970).

\subsubsection{Untersuchungen mit Hilfe von geographischen Informationssystemen}

Um die Populationsstruktur auf dem Tomaros zu untersuchen, wurden Daten an einer Vielzahl von GPS-Punkten erhoben. Dabei handelt es sich um Durchschnittswerte der jeweiligen Merkmale der Individuen, die an den mit GPS markierten Fundorten gemessen wurden. Basierend auf diesen Werten wurden durch Verwendung des geographischen Informationssystems ArcGIS interpolierte Rasterflächen berechnet, die zur Bestimmung der Merkmalsverteilung verwendet wurden. Dadurch war es möglich, die Kontaktzonen zwischen den Populationen zu lokalisieren. Diese Methode wurde von Yanchukov et al. (2006) in einer Bombina-Hybridzone verwendet. Diese Autoren verwendeten jedoch ausschließlich genetische Marker. Mit dieser GIS-basierten Interpolation ist eine exakte Bestimmung der Kontaktzone und somit ebenfalls die Lokalisation der Zentren dieser Kontaktzonen mit morphologischen Parametern möglich. Voraussetzung ist eine relativ hohe Datendichte, um zu große Fehler zu vermeiden. 


\subsection{Faunistische Betrachtung}

\subsubsection{Populationsstruktur der Arten St. rubicundus und St. clavatus}

In der vorliegenden Arbeit wurden Populationen der Arten Stenobothrus clavatus und St. rubicundus miteinander verglichen. Morphologische und ethologische Vergleiche zwischen mehreren inselartigen Populationen führten zu dem Schluss, dass sich die verwendeten St. rubicundus-Populationen Griechenlands nicht nur von der Art St. clavatus sondern ebenfalls von den St. rubicundus der Populationen aus den Alpen und aus Slowenien signifikant unterscheiden. Der Unterschied zur Art St. clavatus ist weitaus größer als zu den nördlichen Populationen dieser Art. Diese Unterschiede werden im Folgenden genauer beschrieben.

\subsubsection{Populationsstruktur von St. rubicundus}

Die geringen, aber signifikanten Unterschiede zwischen den nördlichen und südlichen St. rubicundus-Populationen könnten das Ergebnis einer allopatrischen Trennung und sexueller Selektion sein. Dafür sprechen die Unterschiede im Balzgesang, aber auch die morphologischen Veränderungen der Vorderflügel. Auch ökologische Besonderheiten in den Habitaten der Tiere könnten der Grund sein, warum die Individuen in den nördlichen Populationen einen längeren Femur haben und somit etwas größer sind. Trotz der ethologischen und morphologischen Unterschiede sind beide Populationen durch die Gebirgskette des Balkans miteinander verbunden (Berger et al. 2010), wodurch Genaustausch nicht ausgeschlossen werden kann. Erste Verhaltenstest und Kreuzungsexperimente deuten jedenfalls nicht auf eine prä- oder postzygotische Isolation zwischen den Populationen hin. Die Grenze zwischen den nördlichen und südlichen St. rubicundus Populationen ist nicht bekannt; vermutet wird die Šar Planina, das Grenzgebirge zwischen dem Kosovo und Makedonien (Helversen, pers. Mitt.).

\subsubsection{Populationsstruktur von St. clavatus}

Im Gegensatz zu St. rubicundus ist St. clavatus eine endemische Art, die ausschließlich auf dem Xerovouni und Tomaros zu finden ist. Sie ist erstmals 1978 von Dr. Fer Willemse auf dem Südhang des Tomaros-Südmassivs entdeckt und anschließend von ihm beschrieben 
worden (Willemse 1979). Im Sommer 2007 ist dann eine weitere Population von unserer Arbeitsgruppe auf dem ca. $15 \mathrm{~km}$ entfernten Berg Xerovouni gefunden worden (Sradnick, Klöpfel et al. unveröff.). Die Flügeloberfläche ist im Verhältnis zur Femurlänge halb so groß wie bei St. rubicundus. Die Antennenenden von St. clavatus sind stark verbreitert und dienen höchstwahrscheinlich in der Balz als optisches Signal. Die Balzgesänge der Männchen unterschieden sich ebenfalls stark von denen der Art St. rubicundus.

Die Population des Tomaros und auch des Xerovouni sind jeweils mindesten $10 \mathrm{~km}$ von den benachbarten Bergen entfernt, wo St. clavatus nicht vorkommt. Gründe hierfür könnten unter anderem eine enge Habitatpräferenz, schlechte Flugfähigkeit und spezielle geographische Eigenschaften im Untersuchungsbiet sein, die zu der endemischen Verbreitung dieser Art führen.

\subsubsection{Populationsstruktur auf dem Tomaros}

In Vorarbeiten wurde bereits eine schmale Kontaktzone auf dem Sattel des Südtomaros zwischen St. rubicundus und St. clavatus, beschrieben (Sradnick 2006). Für die Untersuchung der Individuen des Tomaros wurden die hier gesammelten Tiere mit den Individuen der beiden allopatrischen griechischen St. rubicundus- bzw. St. clavatus-Populationen verglichen. Dieser Vergleich zeigte für alle drei Merkmale (Flügel, Antennen und Verhalten) bei den Individuen des Tomaros einen fließenden Merkmalsübergang von der einen zur anderen Art. Dies heißt, dass sowohl Individuen gefunden worden sind, die Merkmale einer der beiden Elternarten aufweisen, aber auch solche mit intermediären Merkmalen. Viele der Tiere zeigten Merkmale, die mit Laborrückkreuzungen übereinstimmten. Tiere, die den F1-Laborkreuzungen entsprachen, waren eher in der Minderheit.

Merkmalveränderungen in der Peripherie der Hybridzone weisen auf Introgression hin. Unter Introgression versteht man den Genaustausch zwischen untereinander hybridisierenden Populationen. Diese wird meist durch sich mit beiden oder einer der Elternarten rückkreuzenden F1-Hybriden vorangetrieben (Anderson und Hubricht 1938). Die Individuen der St. clavatus Population des Tomaros haben im Vergleich zu der allopatrischen Population des Xerovouni signifikant größere Flügel, die in ihrer Form etwas mehr Ähnlichkeiten zu St. rubicundus aufweisen. Bei St. rubicundus des Tomaros hingegen finden sich im Vergleich zu den allopatrischen Populationen etwas verbreiterte Antennenspitzen, die somit der Art St. clavatus ähnlich sind. 
In der Hybridzone zwischen St. clavatus und St. rubicundus könnte sich Introgression wie folgt erklären lassen:

Erstens: Die Nachkommen aus Rückkreuzungen zwischen F1-Hybriden und den Elternarten zeigen in der Morphologie, aber vor allem im Verhalten, bereits große Gemeinsamkeiten zu ihren Elternarten, wodurch eine präzygotische Isolation durch sexuelle Selektion ausgeschlossen wird und Introgression prinzipiell stattfinden kann.

Zweitens: Die in den Parentalpopulationen veränderten Merkmale unterliegen, wenn überhaupt, nur einer geringen Selektion. Begründet ist dies damit, dass die Flügelgröße in der St. clavatus-Population im Balzgesang der Männchen keine Rolle spielt, die Antennen als optisches Signal aber starker sexueller Selektion unterliegen. Der reziproke Fall ist in der St. rubicundus-Population zu beobachten. Hier liegt auf dem Flügelschlag eine starke sexuelle Selektion, weil lauter Flügelschlag ein akustisches und optisches Signal im Balzgesang der Männchen ist (Elsner 1974; Elsner und Wasser 1995b). Verbreiterte Antennen spielen hingegen bei der Balz dieser Art als optisches Signal keine Rolle und unterliegen somit auch keiner direkten Selektion. Letztlich sind für einen Nachweis von Introgression jedoch in jedem Falle molekulargenetische Daten vonnöten (Klöpfel in Vorb.).

Besonders die Veränderung der Vorderflügelmorphologie in den beiden St. clavatusPopulationen des Tomaros führt in der südlichen Population zu einer nahezu kompletten Reduktion des parentalen Merkmals. In der St. clavatus-Population auf dem Nordtomaros ist dieser Effekt nicht so stark ausgeprägt, so dass an den weit von der Kontaktzone entfernten Stellen, z. B. an der Nordspitze des Massivs, viele der Individuen elterliche Merkmale tragen.

Ein Zusammenhang zwischen der morphologischen Veränderung der Individuen im Zentrum der Kontaktzone und dem Verhalten ist anzunehmen, da die betrachteten morphologischen Merkmale (Antennen wie Vorderflügel) vermutlich als optisches Merkmal eine Funktion in der Balz haben und daher der Selektion unterliegen.

Eine Immigration elterlicher Merkmale in das Zentrum der Hybridzone wird als Dispersal bezeichnet. In isolierten Populationen hingegen unterliegt das Dispersal besonderen Ge- 
setzmäßigkeiten. In der Hybridzone des Tomaros kann durch die große Entfernung der allopatrischen Populationen eine Immigration von außerhalb nahezu ausgeschlossen werden. Einzig Merkmale, die Individuen in der Peripherie der Hybridzone besitzen, können immigrieren. Da einige von diesen einer verminderten Selektion unterliegen, kann es zu einem verstärkten Genaustausch zwischen den Elternarten kommen.

\subsubsection{Mosaik-Hybridzone}

Basierend auf morphologischen und ethologischen Merkmalen lässt sich Folgendes über die Gesamtverteilung der Populationen der Arten St. rubicundus und St. clavatus auf dem Tomaros sagen.

Auf dem Nordtomaros sind fast ausschließlich St. clavatus zu finden. Einzig auf kleinen Erhebungen im Süden dieses Berges findet man kleine Populationen von St. rubicundus. Auf dem Nord- und Osthang des Südtomaros befinden sich St. rubicundus, auf dem Südund Westhang St. clavatus. Flügelmorphometrische Vergleiche ergeben bereits leichte Unterschiede zwischen den St. clavatus des Tomaros und des Xerovouni. Eine ähnliche Veränderung ist bei den Individuen der Art St. rubicundus auf dem Tomaros bei der Betrachtung der Antennenbreite zu beobachten. Beide Merkmale zeigen bei jeweils einer der Arten eine leichte Merkmalsveränderung hin zur anderen Art. Dieser Effekt verstärkt sich mit größer werdender Nähe zur Hybridzone.

Drei Kontaktzonen sind zwischen St. rubicundus und St. clavatus auf dem Tomaros zu finden. Jede von ihnen unterscheidet sich in Größe und Aussehen. Kontaktzone I ist die längste der drei. Sie erstreckt sich quer über den Südtomaros in Höhen von $1400 \mathrm{~m}$ bis $1800 \mathrm{~m}$. Besonders viele intermediäre Individuen sind im Zentrum dieser Zone zu finden. Auch die Kline der Flügel- und Antennenmerkmale unterscheiden sich signifikant voneinander (Abb. 98).

Im äußersten Norden des Südtomaros ist die sehr kleine Kontaktzone II zwischen St. rubicundus und St. clavatus lokalisiert. Sie befindet sich am Rand einer kleinen Population von Individuen, die der Art St. clavatus zugeordnet werden. Von allen Seiten ist diese Population von Individuen, die der Art St. rubicundus zugeordnet werden, umgeben.

Auch unterhalb der kleinen Erhebungen im Süden des Nordtomaros treffen die beiden Arten aufeinander (Kontaktzone III). Somit bilden sich an mehreren Stellen kleine Kontakte 
zwischen den Populationen. Da sich aber alle in unmittelbarer Nähe befinden, können sie zu einer Kontaktzone zusammengefasst werden. Sehr steile Hänge im südlichen Teil des Nordtomaros verhindern eine detaillierte Untersuchung einer der beiden Seiten der Kontaktzone. Im Vergleich zu den Klinen im Bereich der Kontaktzone I unterscheiden sich die Kline des Antennen- und Flügelmerkmals nur leicht voneinander.

Es werden zwei Möglichkeiten der Populationsverteilung in diesem Gebiet angenommen: Erstens können St. rubicundus ausschließlich in kleinen von St. clavatus umgebenen Populationen zu finden sein. Dieses würde einer reziproken Verteilung im Vergleich zu Kontaktzone II entsprechen. Zweitens könnten St. rubicundus den nördlichen Bereich des Osthangs des Nordtomaros besiedeln und so eine langgezogene Kontaktzone mit St. clavatus bilden. Dafür spricht, dass St. rubicundus auf den Südtomaros ebenfalls den Osthang besiedelt und so dieses Habitat präferiert.

Eine solche Populationsstruktur wie am Tomaros, hier bestehend aus zwei St. clavatus Populationen und einer dazwischen liegenden St. rubicundus Population, an deren Grenzen Hybridzonen zu finden sind, bezeichnet man als Mosaik-Hybridzone. Vor allem wenn Elternarten verschiedene ökologische Nischen präferieren, kommt es zur Bildung von Hybridzonen dieser Art. Ein ähnliches Beispiel ist die Hybridzone zwischen den beiden Grillenarten Gryllus firmus und G. pennsilvanicus in Nordamerika, die verschiedene Substrate (Böden) präferieren (Harrison und Rand 1989; Ross und Harrison 2002). Auch im Falle der Hybridzone zwischen den beiden Unkenarten Bombina bombina und B. variegata zeigen die Elternarten verschiedene Habitatpräferenzen. Eine der beiden Arten bevorzugt Teiche, die andere kleine Pfützen und Tümpel (MacCallum et al. 1998; Yanchukov et al. 2006). Dadurch lässt sich die Struktur der Hybridzone erklären. Unterschiedliche Habitatpräferenzen der Elternarten erklären auch die Struktur der Hybridzone zwischen den Feldheuschrecken Chorthippus albomaginatus und Ch. oschei (Vedinina 2010). Im Gegensatz hierzu erklären Bridle und Butlin (2002) die Mosaikhybridzone zwischen Chorthippus brunnes und Ch. jakobsi damit, dass durch die Ausbreitung der elterlichen Genotypen in die Zentren der Hybridzonen die dort existierenden Hybridpopulationen an einigen Stellen verschwinden.

Unterschiedliche Habitatpräferenzen der Arten St. rubicundus und St. clavatus in der Hybridzone könnten Grund für die Mosaikstruktur der Hybridzone sein. Die morphologische 
Vermischung beider Arten spricht gegen eine Verdrängung der Hybridpopulationen. für eine unterschiedliche Habitatpräferenz spricht die Beobachtung, dass St. clavatus und St. rubicundus nie sympatrisch vorkommen, spricht.
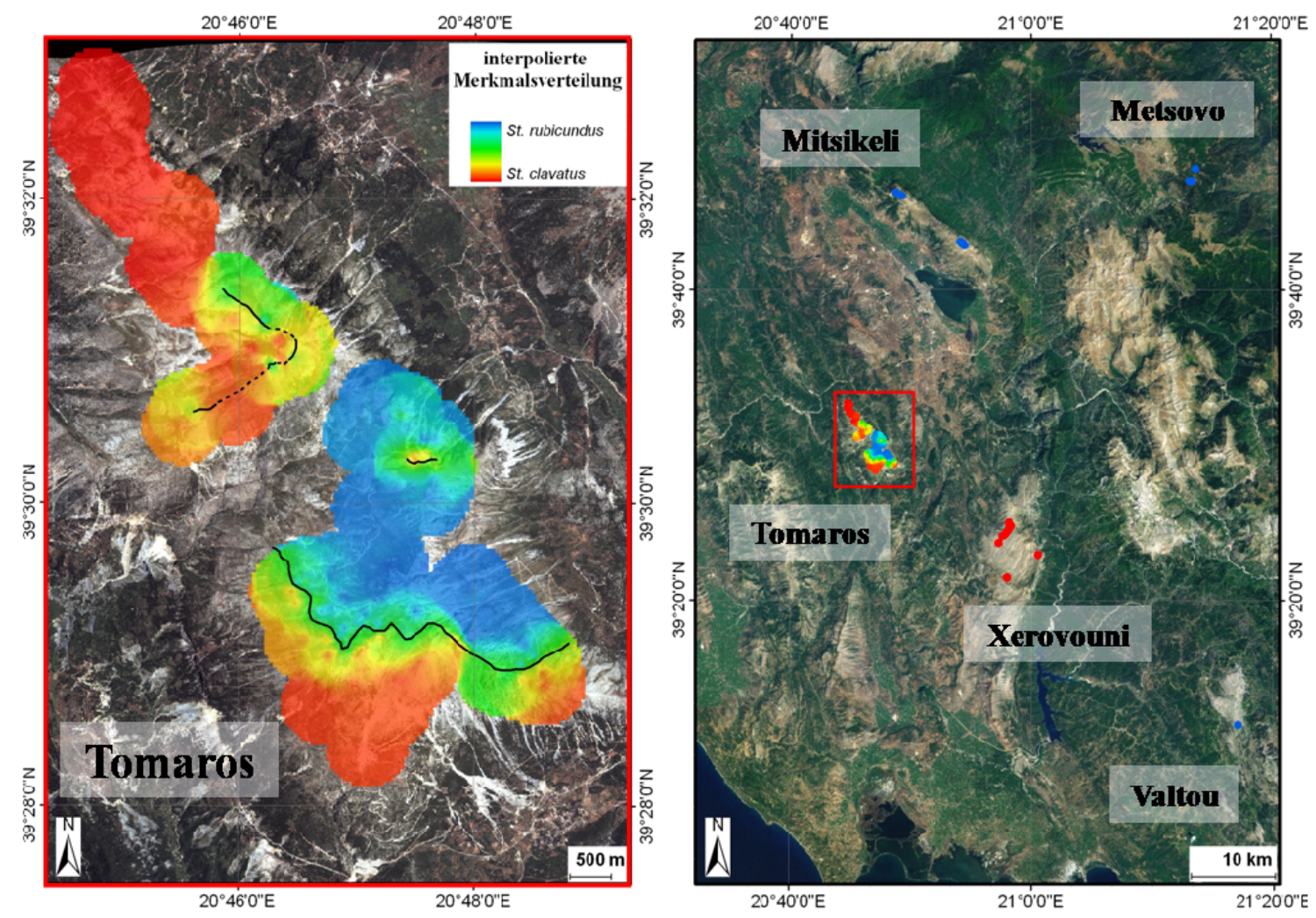

Abb. 108 Populationsverteilung basierend auf allen morphologischen und ethologischen Merkmalen auf dem Tomaros (links) und den umgebenen Bergen (rechts). Rot gekennzeichnete Gebiete sind von St. clavatus und blau gekennzeichnete Gebiete von St. rubicundus.

\subsubsection{Zur Entstehung beider Arten}

Signifikante morphologische und ethologische Unterschiede zwischen den Arten St. rubicundus und St. clavatus, sind aller Wahrscheinlichkeit nach das Produkt allopatrischer Trennung. Diese Trennung ist vor allem bei Individuen, die enge ökologische Nischen besetzen, nicht selten (Knowles 2000). Während interglacialer Perioden spalteten sich gerade in den südlichen Refugien, zu denen auch das Gebiet des heutigen Griechenlands gehört (Tzedakis 1993, Hewitt 1999, Tzedakis et al. 2002), häufig Arten auf. In Warmphasen wurden Populationen durch Besiedelung von Bergen in viele Subpopulationen aufgeteilt. Während der Kaltphasen kam es dann durch Verlassen der Berghabitate erneut zur Vermischung der Subpopulationen (Hewitt 1996; Ciplak 2004). Die Folge dieses sich oft wiederholenden Prozesses ist eine enorme Diversität in den südlichen Refugien. Daher kann auch die Ursprungsart von St. rubicundus und St. clavatus in den kälteren Phasen die Täler und 
in den Warmphasen, in der wir uns beispielsweise momentan befinden, die Berge besiedelt haben. Weitere Hinweise auf allopatrische Speziation zeigen verhaltensbiologische Vergleiche zur Art Stenobothrus werneri (Adelung 1907), die in Westrussland und Asien, aber nicht in Europa zu finden ist. Individuen dieser Art wird aufgrund von Verhaltenmerkmalen dieser Art eine nahe Verwandtschaft zu St. rubicundus und St. clavatus zugesprochen (Berger 2008). Wie auch St. clavatus führen die St. werneri-Männchen hochamplitudige Beinbewegungen zusammen mit komplexen Lauten und Antennenbewegungen aus. Aber im Gegensatz zu St. clavatus haben diese Tiere keine verbreiterten Antennen. Die stufigen Abwärtsbewegungen sind ein plesiomorphes Merkmal einiger Heuschreckengruppen (Elsner 1974a; Elsner und Wasser 1995a). Flügelschläge wie sie St. rubicundus zeigt, gelten als abgeleitet. Somit ist die Dominanz von St. clavatus Elementen im F1 Balzgesang nicht überraschend, weil ursprüngliche Merkmale oft dominant sind und abgeleitete Merkmale häufiger dazu neigen, rezessiv zu sein.

Sexuelle Selektion (West-Eberhard 1983; Barraclough et al. 1995; Eberhard 1996) oder natürliche Selektion (Bernatchez et al. 1996; McCune und Lovejoy 1997; Schluter 1998) können ebenfalls die Herausbildung von unterschiedlichen Merkmalen in allopatrisch getrennten Populationen führen. Allopatrische Trennung allein muss nicht zwangsläufig zur allopatrischen Speziation führen (Knowless 2000). Für allopatrische Speziation durch sexuelle Selektion sprechen die sehr unterschiedlichen und komplexen Gesänge beider Arten sowie die damit verbundenen morphologischen Veränderungen.

\subsubsection{3 Ökologische Nische}

Das inselartige Vorkommen von St. rubicundus und St. clavatus ist durch die Präferenz von speziellen ökologischen Nischen zu erklären. Bei den Habitatpräferenzen spielt neben Temperatur vor allem die Spezialisierung auf Futterpflanzen eine Rolle. In Laborzuchten überleben sowohl St. rubicundus als auch St. clavatus nur mit Gräsern der Gattung Festuca. Wurden Gräser anderer Gattungen verfüttert, starben die Tiere. Im Untersuchungsgebiet findet man ab einer Höhe von 1300 m hauptsächlich Gräser der Gattung Festuca, was erklärt, warum diese Habitate von den Stenobothrus-Arten besiedelt werden.

Klimadaten aus den Jahren 1950-2000, die aus der Datensammlung Worldclim (Hijmans et al. 2005) stammen, weisen ebenfalls auf eine inselartige Verbreitung aufgrund enger ökologischer Nischen hin. Die durchschnittlichen interpolierten Jahrestemperaturen an den 
Fundorten der Individuen der Arten St. rubicundus und St. clavatus lag zwischen $7{ }^{\circ} \mathrm{C}$ und $9^{\circ} \mathrm{C}$ und damit auf diesen Bergen um $2{ }^{\circ} \mathrm{C}$ bis $4{ }^{\circ} \mathrm{C}$ kälter als in den umgebenden Talsohlen. Auch im Sommer und Winter sind Temperaturunterschiede in diesem Bereich gemessen worden. Die Jahresdurchschnittstemperaturen an den Fundorten der nördlichen St. rubicundus-Populationen liegen in der Schweiz bei unter $5^{\circ} \mathrm{C}$ und in Slowenien zwischen $9^{\circ} \mathrm{C}$ und $11^{\circ} \mathrm{C}$. In den Habitaten der beiden untersuchten Arten ist es somit um mindestens $2^{\circ} \mathrm{C}$ kälter als in den niedriger gelegenen Umgebungen (Abb. 109).

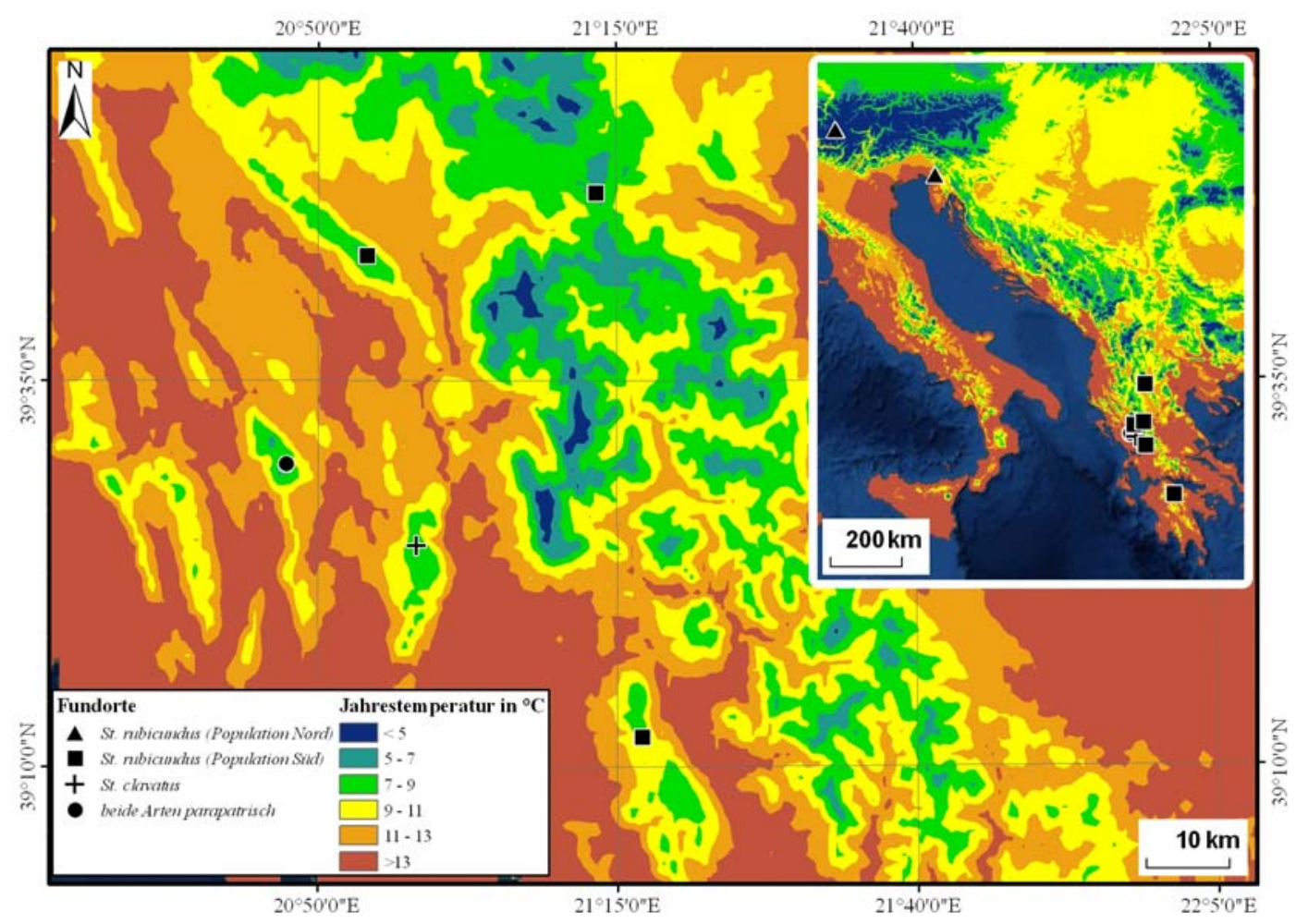

Abb. 109 Meteorologische Karte der Untersuchungsgebiete (Griechenland und Südeuropa) basierend auf den Jahresdurchschnittstemperaturen. Erstellt mit ArcGIS anhand von Daten aus WorldClim. Markiert sind die Fundorte der Stenbothrus-Populationen (siehe Einschaltbild unten links).

In den griechischen Untersuchungsgebieten wurden durchschnittliche Niederschlagsmengen zwischen $900 \mathrm{~mm}$ und $1200 \mathrm{~mm}$ ermittelt (Abb. 110). Insgesamt ist der Osten der ionischen Halbinsel mit durchschnittlichen Niederschlagsmengen von 300-600mm pro Jahr sehr viel trockener als der Westen. Dieses West-Ost-Gefälle ist entlang der gesamten östlichen Adriaküste zu beobachten (siehe kleiner Kartenausschnitt Abb. 110 oben rechts). Vor allem der Winter ist im Westen der ionischen Halbinsel sehr regenreich. Im Sommer hingegen ist der Unterschied zwischen den westlichen und östlichen Gebieten sehr viel geringer. 


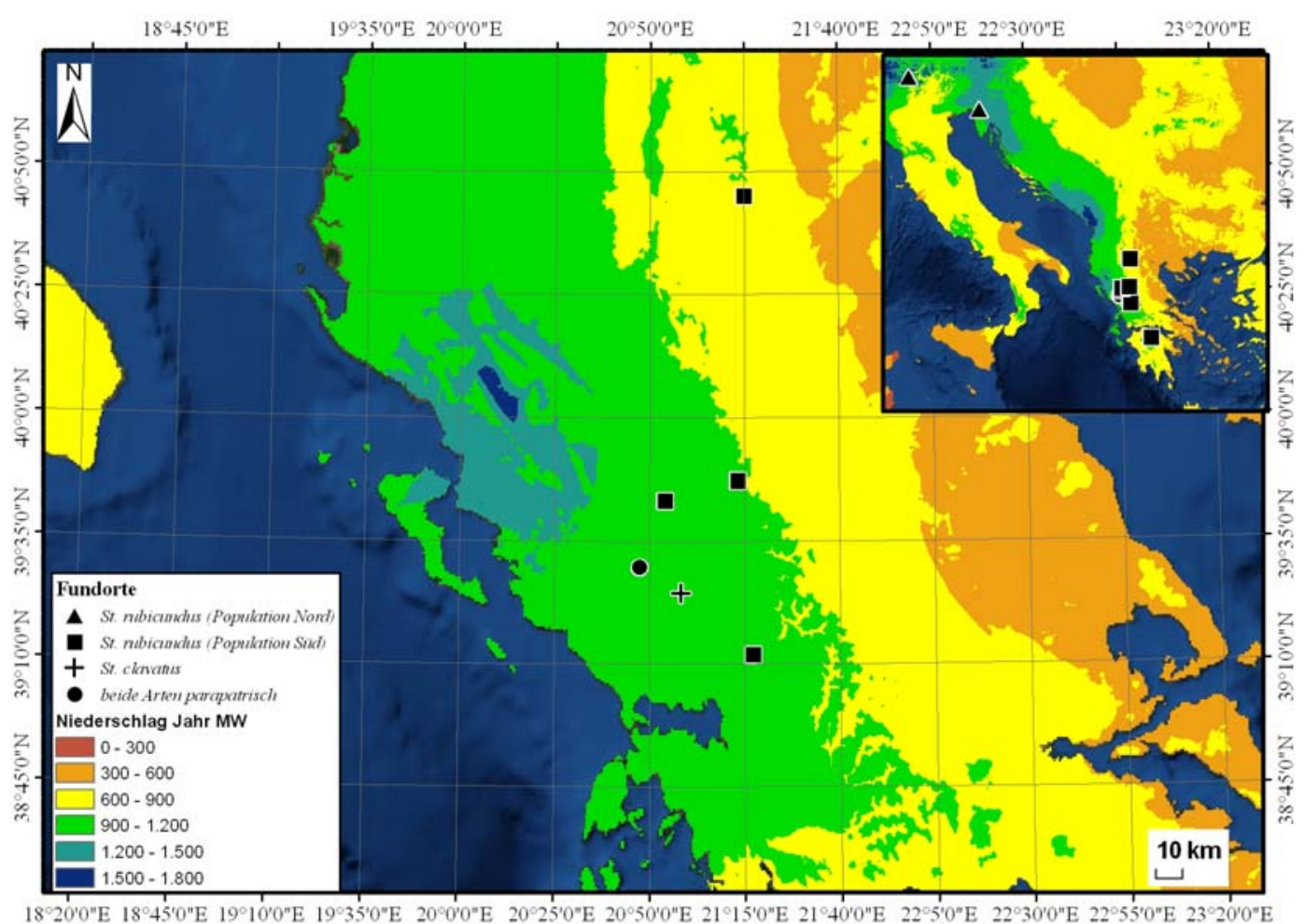

Abb. 110 Meterologische Karte der Untersuchungsgebiete (Griechenland und Südeuropa) basierend auf dem Jahresdurchschnittsniederschlag. Erstellt mit ArcGIS auf Grund von Daten aus WorldClim. Markiert sind die Fundorte der Stenobothrus Populationen (siehe Einschaltbild unten links)

Bei dem Untersuchungsgebiet handelt es sich um Habitate, die weit auseinander liegen, so dass ein momentaner Austausch zwischen diesen ökologischen Nischen oder auch „Inseln“ unmöglich erscheint. Diese seltene Konstellation von Habitaten ermöglicht eine Untersuchung der Arten. Solche Populationen mit inselartiger Struktur eignen sich gut zur Untersuchung allopatrischer Artbildungsprozesse.

\subsubsection{Radiation}

Geologische Untersuchungen von (Tzedakis 2002) in der Umgebung von Ioannina zeigen, dass das Untersuchungsgebiet während des Pleistozän zu den interglacialen Refugien gehörte in denen das Klima in den letzten 430.000 Jahren im Vergleich zu Gebieten Nordeuropas relativ stabil war. In solchen Refugien ist heute eine Vielzahl von endemischen Arten zu finden, zu denen auch St. clavatus gehört. Diese Erkenntnisse legen die Vermutung nahe, dass beide Arten möglicherweise während der letzten Eiszeiten allopatrisch getrennt worden sind. Im Holozän (ca. 11.000 Jahre bis heute) trafen die allopatrischen Arte bzw. Populationen während längerer Wärmephasen wieder aufeinander. Dies könnte auf dem Tomaros der Fall gewesen sein. Hier treffen heute die inselartigen Ausbreitungsgebiete der Arten St. rubicundus und St. clavatus aufeinander. St. rubicundus breitete sich höchstwahrscheinlich ähnlich wie Chorthippus parallelus (Cooper et al. 1995, Hewitt 1999, 2000, 
2004) noch weiter in Richtung Norden aus, St. clavatus hingegen verbleibt endemisch. Der Verbleib von St. clavatus genau in diesem Gebiet kann unter anderem auch mit der besonderen Wetterlage dort zu tun haben. Mit jährlichen Niederschlagsmengen von $900 \mathrm{~mm}$ bis $1.200 \mathrm{~mm}$ gehört dieses Gebiet mit zu den niederschlagreichsten Griechenlands. Dies hängt unter anderem mit dem besonderen Mikroklima, ausgelöst durch Luftströmungen von der Adria, die Regen nach Griechenland tragen, zusammen. Dieser Regen trifft auf die Westküste der ionischen Halbinsel und regnet sich meist vor und über den Bergen (Pindosgebirge) ab, so dass nur noch wenig den Osten der Halbinsel erreicht. Eine solches Mikroklima wie im Nord-westem Griechenlands prädestiniert für Endemiten wie St. clavatus.

\subsubsection{Zukunft der Arten}

Arten, die eine starke Anpassung an kleine ökologische Nischen besitzen, sind immer besonders vom Aussterben bedroht. So ist St. clavatus enorm von den Auswirkungen des Klimawandels und durch die landwirtschaftliche Nutzung des Habitats betroffen. Durch die globale Erwärmung könnten die Festuca-Wiesen und somit auch St. clavatus von Xerovouni und Tomaros verschwinden. Extensive Bewirtschaftung der Wiesen auf den Bergen könnten wichtige Futterpflanzen der Heuschrecken verdrängen und schließlich die Population gefährden. Im Gegensatz zu St. clavatus könnten die Auswirkungen von Klimawandel und Landwirtschaft den Individuen der Art St. rubicundus sehr viel weniger schaden. Dies liegt an den sehr viel größeren Ausbreitungsgebieten dieser Art, die von den Alpen über die Karpaten bis weit in den südlichen Balkan reichen.

\subsection{Evolutionsbiologische Einordnung}

\subsubsection{Präzygotische Isolation}

Dobzhansky (1970) geht davon aus, dass sich Individuen eines Taxons (Population) bevorzugt untereinander, anstatt sich fremd zu paaren. Grundlage dafür ist das selektionsbegünstigte Verhalten der Elternarten gegen die Hybridnachkommen mit geringerer Fitness. Dieses Verhalten würde dazu führen, dass sich die Unterschiede zwischen den beiden betreffenden Arten noch verstärken (Reinforcement), was die allopatrische Speziation noch beschleunigen würde. Hewitt (1993) beschreibt diese Verstärkung durch das Aufeinandertreffen von während der Eiszeiten getrennten Ch. paralleus-Unterarten als postglaciales Rein- 
forcement. Verhaltensexperimente, die eine Paarungsbevorzugung der Elternarten zeigen, ergaben jedoch keine eindeutigen Ergebnisse.

Morphologische und verhaltensbiologische Untersuchungen an Individuen der Hybridzone zwischen St. rubicundus und St. clavatus auf dem Tomaros-Massiv führten zu ähnlichen Ergebnissen. Die starke morphologische und ethologische Vermischung der beiden Arten im Zentrum der Hybridzone sowie Introgression von morphologischen Merkmalen in der Peripherie sprechen dort nicht für Merkmalsverstärkung oder vollständige reproduktive Selektion gegen F1-Hybride (Ritchie at al. 1989). Auch in Auswahlexperimenten von Fähsing (2010) zeigen die Weibchen aus Kontaktzonen, aber auch Labor F1-Hybriden, keine gesteigerte Selektivität gegenüber den Elternarten, aber auch nicht gegen intermediäre Gesänge. Weibchen der Elternarten bevorzugen sogar die teilweise intermediären Gesänge. Es stellt sich nun die Frage, warum es nur in den Zentren der Hybridzone zu hohem Aufkommen von intermediären Merkmalen kommt. In der Peripherie hingegen sind wenige bis keine Individuen mit intermediären Merkmalen zu finden.

Aber welche Mechanismen wirken der Bildung eines Hybridschwarms auf dem Tomaros entgegen? Wie kommt es trotz des Wegfalls der reproduktiven Selektion im Zentrum der Hybridzone nicht zur vollständigen Vermischung? Vor allem die Selektion gegenüber intermediären Gesängen scheint ein wichtiger Faktor zu sein, der einer Ausbreitung des Zentrums der Kontaktzone entgegenwirkt. Die im Vergleich zu den Klinen der morphologischen Merkmale sehr viel steilere Gesangskline lassen die Vermutung zu, dass außerhalb der Kontaktzone eine starke Selektion gegen intermediäre Merkmale wirkt. Der große artspezifische Unterschied zwischen Balzgesängen der Taxa spricht zusätzlich für eine starke präzygotische Isolation in der Peripherie der Hybridzone. Dies deckt sich ebenfalls mit den Präferenzversuchen, in denen die Weibchen in der Peripherie und in den allopatrischen Populationen jeweils die arteigenen Männchengesänge bevorzugten.

Auch ökologische Gesichtspunkte könnten für eine stabilisierte Kontaktzone sprechen. Für verschiedene Habitatpräferenzen der beiden Arten St. rubicundus und St. clavatus sprechen deren parapatrische Verbreitung auf dem Tomaros und ihre strenge Abgrenzung in den allopatrischen Populationen. Vor allem in der Vegetationsdichte gibt es Unterschiede zwischen den bevorzugten Habitaten der beiden Arten (Bergmeyer pers. Mittelung). 
Aber wie kommt es trotz der Gesangsunterschiede zu interspezifischen Paarungen in den Kontaktzonen? Wie wird die präzygotische Isolationsbarriere durchbrochen, wodurch entstehen interspezifische Nachkommen?

Interspezifische Nachkommen sind oft Produkt von veränderter Weibchenwahl. Besonders bei Arten, die komplexe Gesänge mit vielen verschiedenen Elementen produzieren, kann es zu Fehlpaarungen kommen (Bridle und Butlin 2002; Vedenina und Helversen 2003). Folgt man dem Modell von Fishers „run-away-process“ (Fisher 1958; Searcy und Andersson 1986), so präferieren Weibchen besonders extravagante und auffällige Balzgesänge. St. rubicundus produziert lautes Flügelschnarren aber auch St. clavatus zeigt auffällige hochamplitudige Beinbewegungen und zusätzlich rhythmische Antennenbewegungen. Mit diesen zusätzlichen Signalen können die Männchen ihre Attraktivität gegenüber den Weibchen steigern, laufen aber auch Gefahr, Fitnessnachteile durch diese Merkmale zu bekommen.

Das Vorhandensein von komplizierten Balzelementen der Männchen unterstützt die Weibchen, Männchen zu finden die „gute Gene“ haben (Hamilton und Zuk 1982; Zahavi 1987; Maynard Smith 1991). Ist also ein Männchen in der Lage außergewöhnliche Elemente wie lauter Flügelschlag bei St. rubicundus oder Antennenschlag zusammen mit komplexen Beinbewegungen (Phase III St. clavatus) zu produzieren, kann dies ein Anzeichen auf besonders gute genetische Qualität sein.

Um es zu einer Kopulation kommen zu lassen, wählt das Weibchen in der Regel nicht nur arteigene Männchen, sondern prüft diese auch auf qualitative Eigenschaften (Andersson 1994). Somit können einige Gesangsparameter, die bei beiden Arten zu finden sind, weitere Selektionssignale sein. Zwischen St. rubicundus und St. clavatus findet man ebenfalls solche Signale. Dabei handelt es sich um die Flügelschläge, die in bestimmten Abständen zwischen Beinbewegungen eingestreut werden. Die Frequenz dieser beiden Elemente ist nach Elsner und Wasser (1995b) sehr ähnlich und liegt bei 60 bis $70 \mathrm{~Hz}$. Dieses Signal könnte Auslöser für intraspezifische Kopulationen sein. Ähnliches wird auch bei der Hybridisierung von Ch. albmaginatus und Ch. oschei vermutet (Vedenina et a. 2007). Demnach sind in den Balzgesängen Signale zu finden, die zum einen die Artzugehörigkeit, zum anderen die Männchenkonstitution signalisieren (Vedenina 2005). 
In Verhaltensanalysen sind bei Feld-, aber auch bei Laborhybriden, neue Gesangselemente zu finden, die aus elterlichen Elementen zusammengesetzt sind. Oft bestehen diese Gesangselemente aus Serien komplexer, aus mehreren Pulsen zusammengesetzten stufigen Beinbewegungen, die durch einfache Stridulationspulse oder Flügelschläge unterbrochen werden. Aber auch gleichzeitiger Flügelschlag bei großamplitudigen Beinbewegungen (Phase III St. clavatus) sind zu finden. Solch neue Elemente sind auch in der Hybridzone zwischen Ch. albmaginatus und Ch. oschei zu finden. Es ist denkbar, dass neue Gesangselemente sich positiv auf den Paarungserfolg einiger F1 Männchen auswirken könnten.

\subsubsection{Postzygotische Isolation}

Reproduktive Isolation tritt meist dann auf, wenn nach erfolgter interspezifischer Paarung die Hybride eine verminderte Lebensfähigkeit aufweisen. Diese kann durch partielle Inkompatibilität der beiden Genome oder durch verringerte Fitness der intermediären Individuen in den jeweiligen ökologischen Nischen der Elternarten auftreten. Dieser Fall von Fitnessnachteil der F1-Hybriden wird in der Literatur als Haldanesche Regel bezeichnet, die besagt, dass in der F1-Generation der heterozygotische Typ, also meist die Männchen, steril ist und selten, oder erst gar nicht, entsteht (Haldane 1922).

Um diese Frage zu klären, wurden im Labor interspezifische Kreuzungen zwischen Vertretern der jeweiligen Art durchgeführt. Trotz der großen Gesangsunterschiede zwischen den beiden Arten ist es verhältnismäßig einfach, Laborhybride zwischen den Arten St. rubicundus und St. clavatus zu erzeugen. Hierbei ist aber zu erwähnen, dass nur bei der Kreuzung von St. clavatus $q \mathrm{x}$ St. rubicundus $\widehat{\jmath}$ problemlos Nachkommen entstanden sind. Beim reziproken Kreuzungstyp war es lange Zeit nicht möglich F1-Hybride zu erhalten. Einzig Nachkommen aus der Paarung von St. rubicundus von Valtou-Gebirge und einem St. clavatus brachte F1-Hybriden hervor. Bei all diesen F1-Hybriden wurden keine offensichtlichen Fitnessnachteile beobachtet. Auch die F1-Hybride des ersten Kreuzungstyps sind fertil, denn sie brachten lebensfähige Nachkommen (F2-Hybride) hervor.

In der Literatur gibt es einige Beispiele von fertilen F1-Hybriden, die unter Laborbedingungen gezogen worden sind, vor allem unter Vertretern der Gattung Chortippus (Perdeck 1957, Helversen \& Helversen 1975; Stumpner \& Helversen 1994). Von einigen der Arten dieser Gattung, die in der Natur hybridisieren, sind ebenfalls fertile F1 Laborhybride erzeugt worden (Ragge 1981, 1984; Ingrisch 1995, Saldamando et al. 2005, Vedinina 2007). 
Trotz der offensichtlichen Fertilität der Hybrid-Männchen und -Weibchen fand Fähsing (2010), dass arteigene Gesangsmuster von den Weibchen der Elternarten gegenüber intermediären signifikant bevorzugt werden. Die Hybridweibchen sind unselektiv und beantworten alle Gesänge ähnlich häufig. Daraus kann man schließen, dass die Hybridmännchen einen Fitnessnachteil gegenüber den „reinen“ Männchen haben. Somit kann man vom Gesang von einer unvollständigen postzygotischen Isolationsbarriere sprechen.

Es wird davon ausgegangen, dass die Männchen der ersten Hybridgeneration eine reduzierte Fitness im Balzgesang aufweisen, weil die Weibchen nicht Selektion gegen intermediären Gesang zeigen (Fähsing 2010). Somit existiert eine unvollständige postzygotische Isolationsbarriere. Da die F1-Weibchen vollkommen unselektiv sind, heißt das, dass es sich um eine Selektion gegen Hybride handelt, die nicht besonders stark ist, aber eine komplette Vermischung der Taxa verhindert.

Nur sehr wenige Nachkommen gingen aus Kreuzungen von St. rubicundus Weibchen mit St. clavatus Männchen hervor. Es ist zu vermuten, dass z. B. Spermien in dieser Konstellation nicht kompatibel sind und somit eine postzygotische Isolationsbarriere wirkt.

\subsection{Fazit}

Hybridzonen zwischen Populationen mit großen morphologischen und ethologischen Unterschieden, wie sie hier herrschen, sind in der Natur durchaus selten. Im Falle des Tomaros kommt hinzu, dass die Hybridzone zwischen St. rubicundus und St. clavatus durch die geringe Größe und geographische Isolation nahezu einzigartig ist. Prä- und postzygotische Isolationsmechanismen wirken im Zentrum der Hybridzone nur bedingt, verstärken sich aber in der Peripherie. Aber auch unterschiedliche ökologische Habitatpräferenzen können die Mosaikstruktur der Hybridzone erklären.

In der Hybridzone sind bisher nur wenig Anzeichen voranschreitender Artbildung zu erkennen. Jedoch könnten auf dem Tomaros die geographische Isolation und der anhaltende Genfluss zwischen den Populationen in fernerer Zukunft zur parapatrischen oder allopatrischen Speziation führen. Butlin (1998) sieht Hybridzonen eher als ein Fenster auf ein intermediäres Stadium der allopatrischen Artbildung als ein Getriebe für parapatrische Speziation. Aber gerade in Zeiten des klimatischen Wandels können Hybridzonen ein Reser- 
voir von genetischer Vielfalt sein aus dem sich die Individuen bzw. Populationen während starker äußerer Veränderungen bedienen können.

\subsection{Ausblick}

Die Hybridzone zwischen St. clavatus und St. rubicundus konnte detailliert beschrieben und die Hybride exakt eingeordnet werden. Es bleiben aber noch einige Fragen offen:

In morphologischen Untersuchungen sind Anzeichen für eine Introgression zwischen der St. clavatus- und St. rubicundus-Population in der Hybridzone gefunden worden. Es sind aber vor allem molekularbiologische Untersuchungen nötig, um eine Introgression fundiert nachweisen zu können. Ebenso können durch genetische Vergleiche zwischen den möglicherweise nächstverwandten Arten St. cotticus und St. werneri offenstehende Fragen zur Entstehung der Arten St. rubicundus und St. clavatus beantwortet werden.

Die Wirkung von Isolationsmechanismen ist zu großen Teilen noch ungeklärt. Hierzu sind Verhaltenstests erforderlich, in denen die Präferenzen der Weibchen gegenüber dem Werbeverhalten arteigener bzw. artfremder Männchen sowie gegenüber Hybriden geprüft werden. Präferenzexperimente, die akustische, aber auch optische Signale beinhalten, sind unumgänglich. Zudem ist die Frage nach der Wirkung von olfaktorischen Signalen bei der Partnerwahl dieser Heuschreckenarten noch völlig unerforscht.

Es wird vermutet, dass eine unterschiedliche Habitatpräferenz einen großen Einfluss auf die Struktur der Hybridzone hat. Hierzu sind eingehende ökologische Untersuchungen der Heuschreckenhabitate und der Kontaktzonen noch durchzuführen. Im Zuge dessen müßte auch geklärt werden, welche Faktoren ein sympatrisches Vorkommen der Arten verhindern.

Die Hybridzone auf dem Tomaros eignet sich besonders gut, um den Einfluss des Klimawandels auf isolierte Populationen zu untersuchen. In langjährigen Studien könnte somit der Einfluss des Klimawandels auf die allopatrischen aber auch parapatrischen Populationen der beiden Arten betrachtet werden. 


\section{Zusammenfassung}

- Die Feldheuschrecken Stenobothrus clavatus und St. rubicundus, die sich morphologisch und ethologisch stark voneinander unterscheiden, besiedeln mehrere Areale auf dem Nord- und Südmassiv des in Nordwestgriechenland nahe Ioannina gelegenen Tomaros-Gebirges. Es gibt Hinweise darauf, dass die beiden Arten während der letzten Eiszeiten durch allopatrische Speziation getrennt worden sind. Sie treffen nunmehr einzig auf dem Tomaros wieder aufeinander.

- Zwei St. clavatus-Populationen, eine auf dem Nordmassiv und eine auf dem Südhang des Südtomaros umrahmen die St. rubicundus-Population. Diese Populationen grenzen an drei Stellen aneinander und bilden eine Mosaik-Hybridzone

- Die vorliegende Dissertation hatte das Ziel, diese beiden Arten und deren Hybriden hinsichtlich ihrer Morphologie und ihres Verhaltens quantitativ miteinander zu vergleichen sowie ihre Verbreitungsgebiete auf dem Tomaros faunistisch zu erfassen. Damit soll ein Beitrag zur Evolutionsbiologie der Feldheuschrecken geleistet werden.

- Für diesen Vergleich standen allopatrische Populationen, die ohne Kontakt zur jeweils anderen Art sind, auf anderen griechischen Bergen sowie, im Falle von St. rubicundus, auch in Slowenien und der Schweiz zur Verfügung. Die einzige Vergleichpopulation von St. clavatus, einer endemischen Art, wurde im Zuge der vorliegenden Arbeit auf dem ca. 20 km südöstlich des Tomaros gelegenen XerovouniGebirge entdeckt.

- Die morphologischen und ethologischen Vergleiche ergaben nicht nur signifikante Unterschiede zwischen den allopatrischen Populationen von St. rubicundus und St. clavatus, sondern auch Unterschiede zwischen den alpin-dalmatinischen und den griechischen St. rubicundus-Populationen.

- Es zeigte sich, dass bei vielen Tomaros-Individuen der Art St. rubicundus die Antennen gegenüber denen der allopatrischen Populationen leicht verbreitert sind und 
somit, wenn auch nur in schwacher Form, Merkmale von St. clavatus aufweisen. Umgekehrt haben Individuen von St. clavatus des Tomaros gegenüber denen des Xerovouni größere und in der Form veränderte Vorderflügel, die leichte Ähnlichkeiten mit den Flügeln von St. rubicundus aufweisen. In die gleiche Richtung weisen kleinere Änderungen der Werbegesänge beider Arten.

- Die Gesänge der Hybriden zeichnen sich durch eine große intra- und interindividuelle Kompklexität aus. Einige der Feldhybriden, aber auch Laborhybride, zeigen neue Gesangselemente die sich aus einzelnen Parametern der elterlichen Gesänge zusammensetzen.

- Die morphologischen und ethologischen Merkmalsveränderungen bei den Tomaros-Individuen der beiden Arten sind Hinweise für Genaustausch und Introgression zwischen den Populationen. 


\section{Literaturverzeichnis:}

Abramoff M.D., Magelhaes P.J., Ram, S.J. (2004). Image processing with ImageJ. Biophotonics International 11: 36-42.

Anderson E., Hubricht L. (1938). Hybridization in Tradescantia. III. The evidence for introgressive hybridization. Am. J. Bot. 25:396-402.

Andersson M. (1994). Sexual Selection. Princeton Univ. Press, Princeton.

Auffray J.C., Debat V., and Alibert P. (1999). Shape asymmetry and developmental stability. Pp. 309-324 in M. A. J. Chaplain, G. D. Singh, and J. C. McLachlan, eds. On growth and form: spatio-temporal pattern formation in biology. John Wiley and Sons Ltd., Chichester, U.K.

Bailey R.I., Thomas C.D., Butlin R.K. (2004). Premating barriers to gene exchange and their implications for the structure of a mosaic hybrid zone between Chorthippus brunneus and C. jacobsi (Orthoptera: Acrididae). J Evol Biol 17: 108-119.

Barluenga M., Stolting K.N., Salzburger W., Muschick M., Meyer, A. (2006). Sympatric speciation in Nicaraguan crater lake cichlid fish. Nature, 439: 719-723.

Barraclough T.G., Harvey P. H., Nee S. (1995). Sexual selection and taxonomic diversity in passerine birds. Proc. Royal. Soc. Lond. B Biol. Sci. 259:211-215.

Barton N.H. (1983). Multilocus clines. Evolution 37:454-71.

Barton N.H. (2001). Speciation. Trends in Ecology and Evolution, 16: 325-413.

Barton N.H., Hewitt G.M. (1985). Analysis of hybrid zones. Annual Review of Ecology and Systematics 16:113-148.

Barton N.H., Hewitt G.M. (1989). Adaptation, speciation and hybrid zones. Nature 341: 497-503. 
Batcheler C.L. (1967). Preliminary observations of alpine grasshoppers in a habitat modified by deer and chamois. Proceedings New Zealand Ecological Society 14: 15-26.

Bellmann H. (1993): Heuschrecken: beobachten-bestimmen. — Naturbuch Verlag, 2:349 Augsburg.

Berger D. (2008). The evolution of complex courtship songs in the genus Stenobothrus Fischer, 1853 (Orthoptera, Caelifera, Gomphocerinae). Ph.D. Thesis, University of Erlangen-Nuernberg, Germany.

Berger D., Chobanov D.P., Mayer F. (2010). Interglacial refugia and range shifts of the alpine grasshopper Stenobothrus cotticus (Orthoptera: Acrididae: Gomphocerinae). Org Divers Evol 10:123-133.

Bernatchez L., Vuorinen J.A., Bodaly R.A., Dodson J.J. (1996). Genetic evidence for reproductive isolation and multiple origins of sympatric trophic ecotypes of whitefish (Coregonus). Evolution 50:624-635.

Bischoff I., Schröder, S., Misof B. (2009). Differentiation and range expansion of North American squash bee populations (Peponapis pruinosa, Apidae, Hymenoptera) assessed by geometric wing morphometry. Annals of the Entomogical Society of America.

Bishop C. (1995). Neural Networks for Pattern Recognition. Oxford: University Press.

Bookstein F.L. (1991). Morphometric tools for landmark data: geometry and biology. New York: Cambridge University Press. 455 pp.

Bray H.J., Maxwell S.E. (1985). Multivariate Analysis of Variance. Beverly Hills, CA: Sage Publications, Inc. Cohen.

Bridle J.R., Baird S.J., Butlin R.K. (2001). Spatial structure and habitat variation in a grasshopper hybrid zone. Evolution 55: 1832-1843. 
Bridle J.R., Butlin R.K. (2002). Mating signal variation and bimodality in a mosaic hybrid zone between Chorthippus grasshopper species. Evolution 56:1184-1198.

Bruner E., Manzi G., (2001). Allometric analysis of the skull in Pan and Gorilla by geometric morphometrics. Riv. Antropol. (Roma) 79: 45-52.

Buckley S.H., Tregenza T., Bultin R.K. (2003). Transitions in cuticular composition across a hybrid zone historical accident or environmental adaption? Biol. J. Linn. Soc. 78: 193-201.

Buño I., Lopez-Fernandez C., Butlin R. K., Hewitt G. M., Gosalvez J. (1994) A hybrid zone between two subspecies of the grasshopper Chorthippus parallelus along the Pyrenees: the west end. Heredity 73: 625-634.

Butlin R.K., Tregenza T. (1998). Levels of genetic polymorphism: marker loci versus quantitative traits. Phil. Trans. Roy. Soc. Lond. B 353:187-198.

Chopard L. (1951). Faune de France. Vol. 56, Orthopteroides. 359 pp. Paris: Lechevalie.

Ciplak B. (2004). Biogeography of Anatolia: the marker group Orthoptera. Mem. Soc. Entomol. Ital. 82: 357-372.

Clabaut C., Bunje P.M., Salzburger W., Meyer. A. (2007) Geometric morphometric analyses provide evidence for the adaptive character of the Tanganyikan cichlid fish radiations. Evolution: International Journal of Organic Evolution 61: 560-578.

Cooper S.J., Ibrahim K.M., Hewitt G.M. (1995). Postglacial expansion and genome subdivision in the European grasshopper Chorthippus parallelus. Molecular Ecologie. 4: 49-60.

Cornuet J.-M., Piry S., Luikart G., Estoup A. Solignac M. (1999). New Methods Employing Multilocus Genotype to Select or Exclude Populations as Origins of Individuals. Genetics 153: 1989-2000. 
Coyne J.A., Orr H. A. (1989b). Patterns of speciation in Drosophila. Evolution 43: 362381.

Coyne J.A., Orr H.A. (1997). "Patterns of speciation in Drosophila" revisited. Evolution 51: 295-303.

Coyne J.A., Orr H.A. (2004). Speciation. Sinauer Associates, Inc., Sunderland, MA.

Cracraft J. (1989). Speciation and its ontology: the empirical consequences of alternative species concepts for unterstanding patterns and processes of differentiation. In: Speciation and Its Consequences (eds Otte D, Endler JA), pp. 28-59. Sinauer Associates, Sunderland, MA.

Darwin C. (1859). The origin of species. New York: Modern Library.

De la Riva J., Le Pont F., Ali V., Matias A., Mollinedo S., Dujardin J.P. (2001). Wing geometry as a tool for studying the Lutzomyia longipalpis (Diptera: Psychodidae) complex. Mem. Inst. Oswaldo Cruz 96: 1089-1094.

DeMers M.N. (2000). Fundamentals of Geographic Information Systems. John Wiley and Sons, Inc., New York.

De Queiroz K., Donoghue M.J. (1988). Phylogenetic systematics and the species problem. Cladistics 4: 317-338.

Dieckmann U., Doebeli M. (1999). On the origin of species by sympatric speciation. Nature 400: $354-357$.

Dillon R.T.Jr., Manzi. J.J. (1989). Genetics and shell morphology in a hybrid zone between the hard clams Mercenaria mercenaria and M. campechiensis. Mar. Bioi. 100:2 1722. 
Dobzhansky T. (1970). Genetics of the evolutionary process. Columbia University Press, New York.

Dres M., Mallet J. (2002). Host races in plant-feeding insects and their importance in sympatric speciation. Philosophical Transactions of the Royal Society of London, Series B, 357: 471-492.

Dryden I.L., Mardia, K.V., (1998). Statistical Shape Analysis. Wiley, New York.

Eberhard W.G. (1996). Female control: sexual selection by cryptic female choice. Princeton Univ. Press, Princeton, NJ.

Efron B. (1979). Bootstrap methods: another look at the jackknife. Ann. Statist. 7: 1-26.

Elsner N. (1974a). Neural economy: Bifunctional muscles and common central pattern elements in leg and wing stridulation in the grasshopper Stenobothrus rubicundus Germ. J. comp. Physiol. 89: 227-236.

Elsner N. (1974b). Neuroethology of sound production in gomphocerine grasshoppers (Orthoptera: Acrididae). I. Song patterns and stridulatory movements. J. comp. Physiol. 88, 67-102.

Elsner N., Klöpfel A., Sradnick J. (2009). Das geschmähte fremde Blut - Bastardierung als Motor der Evolution . Evolution - Zufall und Zwangsläufigkeit der Schöpfung. Wallenstein Verlag: 191-232.

Elsner N., Wasser, G. (1995a). The transition from leg to wing stridulation in two geographically distinct populations of the grasshopper Stenobothrus rubicundus. Naturwissenschaften 82: 384-386.

Elsner N., Wasser G. (1995b). Leg and wing stridulation in various populations of the gomphocerine grasshopper Stenobothrus rubicundus (Germar 1817). I. Sound patterns and singing movements. Zoology 98: 179-190. 
Elsner N., Wasser G. (1995c). Leg and wing stridulation in various populations of the gomphocerine grasshopper Stenobothrus rubicundus (Germar 1817). II. Neuromuscular mechanisms. Zoology 98: 191-199.

Faber A. (1953). Laut- und Gebärdensprache bei Insekten: Orthoptera (Geradflügler). Staatl Mus Naturk Stuttgart.

Fähsing S. (2010). Intra- und interspezifische sexuelle Selektion bei zwei Feldheuschreckenarten und deren Hybriden - Eine evolutionsbiologische Studie - (Diplomarbeit; Universität Göttingen).

Fisher R.A. (1958). The Genetical Theory of Natural Selection. The Clarendon Press, Oxford. Second Revised Edition, Dover Press, New York.

Francoy T.M., Wittmann D., Drauschke M., Müller S., Steinhage V., Becerra-Laure M.A.F., De Jong D., Gonçalves L.S. (2008). Identification of Africanized honey bees through wing morphometrics: two fast and efficient procedures. Apidologie 39: 488494.

Francuski L., Ludoski J., Vujić A., Milankov V. (2009). Wing geometric morphometric inferences on species delimitation and intraspecific divergent units in the Merodon ruficornis group (Diptera, Syrphidae) from the Balkan Peninsula. Zoological Science 26: $301-308$.

Frost S.R., Marcus L.F., Bookstein F.L., Reddy D.P., Delson E. (2003). Cranial allometry, phylogeography, and systematics of large-bodied Papionins (Primates: Cercopithecinae) inferred from geometric morphometric analysis of landmark data. Anat. Rec. 275A: 1048-1072.

Fry J.D. (2003). Multilocus models of sympatric speciation: Bush versus Rice versus Felsenstein. Evolution 57: 1735-1746.

Futuyma D.J, Mayer G.C. (1980) Non-allopatric speciation in animals. Systematic Zoology 29: 254-271. 
Garnier, S., Magniez-Jannin F, Rasplus J.-Y., Alibert P. (2005). When morphometry meets genetics: inferring the phylogeography of Carabus solieri using Fourier analyses of pronotum and male genitalia. J. Evol. Biol. 18: 269-280.

Gavrilets S., Hastings A., (1996). Founder effect speciation: a theoretical reassessment. American Naturalist, 147: 466-491.

Genner M.J., Seehausen O., Cleary D.F.R., Knight M.E., Michel E., Turner, G.F. (2004). How does the taxonomic status of allopatric populations influence species richness within African cichlid fish assemblages? J. Biogeogr. 31: 93-102.

Goodall C.R. (1991). Procrustes methods in the statistical analysis of shape. J R Stat Soc B 53: 285-339.

Gottsberger B., Mayer F. (2007). Behavioral sterility of hybrid males in acoustically communicating grasshoppers (Acrididae, Gomphocerinae). J. Comp. Physiol. A 193: 703-714.

Gottsberger B. (2008). Interspecific hybridization between the grasshoppers Chorthippus biguttulus and C. brunneus (Acrididae; Gomphocerinae). Dissertation, Natuwiss.. Fak. II Univ. Erlangen.

Gower, J.C. (2001). Procrustes Analysis. In: International Encyclopedia of the Social and Behavioral Sciences. Eds. Smelser N. J. Baltes P. B.. Pergamon: Amsterdam etc. 12141-12143.

Grant B.R., Grant P.R., (1996a). Cultural inheritance of song and its role in the evolution of Darwin's finches. Evolution 50: 2471-2487.

Grant B.R., Grant P.R. (1996b). High survival of Darwin's finch hybrids: effects of beak morphology and diets. Ecology 7: 500-509. 
Grant B.R., Grant P.R. (1998). Hybridization and speciation in Darwin's finches: the role of sexual imprinting on a culturally transmitted trait. In Endless forms: species and speciation (eds. Howard D.J. and Berlocher S.H.). 404-422. New York, NY: Oxford University Press.

Grant P.R., Grant B.R., Petren K. (2005). Hybridization in the recent past. Am. Nat. 166: 56-67.

Haldane J.B.S. (1922). Sex ratio and unisexual sterility in hybrid animals. J. Genet 12: 101- 109.

Hamilton W., Zuk M. (1982). Heritable true fitness and bright birds: a role for parasites? Science 218:384-387.

Harrison R.G. (1986). Pattern and process in a narrow hybrid zone. Heredity 56: 337-349.

Harrison R.G. (1990). Hybrid zones: windows on the evolutionary process. Oxford Surveys in Evolutionary Biology 7: 69-128.

Harrison R.G. (1993). Hybrids and hybrid zones: Historical perspective. In: Harrison R.G., ed. Hybrid zones and the evolutionary process. Oxford University press: 3-12.

Harrison R.G., Rand D.M. (1989). Mosaic hybrid zones and the nature of species boundaries. Pp. 111-133 in Otte D., Endler J. A., (eds.). Speciation and its consequences. Sinauer, Sunderland, Massachusetts, USA.

Harz K. (1957). Die Geradflügler Mitteleuropas. - Fischer, Jena, 494 pp.

Helversen D.v. (1972): Gesang des Männchens und Lautschema des Weibchens bei der Feldheuschrecke Chorthippus biguttulus (Orthoptera, Acrididae). J. comp. Physiol. I. 81: $381-422$

Helversen D.v., Helversen O.v. (1975a). Verhaltensgenetische Untersuchungen am akustischen Kommunikationssystem der Feldheuschrecken (Orthoptera, Acrididae) I. Der 
Gesang von Artbastarden zwischen Chorthippus biguttulus und Ch. mollis. Journal of Comparative Physiology 104: 273-299.

Helversen D.v., Helversen O.v. (1975b). Verhaltensgenetische Untersuchungen am akustischen Kommunikationssystem der Feldheuschrecken (Orthoptera, Acrididae) II. Das Lautschema von Artbastarden zwischen Chorthippus biguttulus und Ch. mollis. Journal of Comparative Physiology 104: 301-323.

Helversen O.v., Helversen D.v. (1994). Forces driving coevolution of song and song recognition in grasshoppers. In: Schildberger K., Elsner N. (eds) Neural basis of behavioural adaptations. Fortschr. Zool. 39: 253-284.

Helversen O.v. (1986). Gesang und Balz bei Feldheuschrecken der Chorthippus albomarginatus-Gruppe (Orthoptera: Acrididae) Zool Jahrb Syst 113:319-242.

Helversen O.v., Elsner N. (1977). The stridulatory movements of acridid grasshoppers recorded with an opto-electronic device. J Comp Physiol 122: 53-64.

Hewitt G.M. (1988). Hybrid zones - natural laboratories for evolutionary studies. Trends Ecol. Evol. 3:158-167.

Hewitt G.M. (1993). Postglacial distribution and species substructure: lessons from pollen, insects and hybrid zones. In: Lees, D. R. and Edwards, D. (eds) Evolutionary Patterns and Processes, pp. 97-123. Linnaean Society of London, Academic Press, London.

Hewitt G.M. (1996). Some genetic consequences of ice ages, and their role in divergence and speciation. Biol. J. Linn. Soc. 58: 247-276.

Hewitt G.M. (1999). Post-glacial recolonization of European biota. Biol. J. Linnean Soc. 68: 87-112.

Hewitt G.M. (2000). The genetic legacy of the Quaternary ice ages. Nature 405: 907-913. 
Hewitt G.M. (2001). Speciation, hybrid zones and phylogeography: or seeing genes in space and time. Mol. Ecol. 10: 537-549.

Hewitt G.M. (2004). Genetic consequences of climatic oscillations in the Quaternary. Phil. Trans. R. Soc. Lond. B 359: 183-195.

Hijmans R.J., Cameron S.E., Parra J.L., Jones P.G., Jarvis A. (2005) Very high resolution interpolated climate surfaces for global land areas. International Journal of Climatology 25: 1965-1978.

Hjelm J., Svanbäck R., Byström P., Persson L., Wahlström E. (2001). Diet-dependent body morphology and ontogenetic reaction norms in Eurasian perch. Oikos 95: 311-323.

Howard D.J. (1986). A zone of overlap and hybridization between two ground cricket species. Evolution 40: 34-43.

Howard D.J., Berlocher S.H. (1998). Endless Forms: Species and Speciation. Oxford University Press, Oxford.

Ingrisch S. (1995). Evolution of the Chorthippus biguttulus group (Orthoptera, Acrididae) in the Alps based on morphology and stridulation. Rev. Suisse Zool. 100: 475-535.

Jacobs W. (1953). Verhaltensbiologische Studien an Feldheuschrecken. Beiheft 1 Z Tierpsych.

Jacobson G.C.R., Bianchi V.L. (1902). Orthoptera and Pseudoneuroptera of the Russian Empire: 317. St. Petersburg.

Jiggins, C.D. et al. (1997) The maintenance of species differences across a Heliconius hybrid zone. Heredity 79, 495-505.

Jiggins C.D., Mallet J. (2000). Bimodal hybrid zones and speciation. Trends Ecol Evol 15 (6): 250-255. 
Jones R.M., Searle J.B. (2003). Mapping the course of the Oxford-Hermitage chromosomal hybrid zone in the common shrew Sorex araneus - a GIS approach. Mammalia 67: $193-200$.

Kawecki T.J. (1996). Sympatric speciation driven by beneficial mutations. Proceedings of the Royal Society of London. Series B. 263: 1515-1520.

Kawecki T.J. (1997). Sympatric speciation via habitat specialization driven by deleterious mutations. Evolution, 51: 1751-1763.

Kirkpatrick M. and Servedio M.R. (1999). The reinforcement of mating preferences on an island. Genetics 151, 865-884.

Knowles L.L. (2000). Tests of Pleistocene speciation in montane grasshoppers (genus Melanoplus) from the Sky Islands of western North America. Evolution 54:1337- 48.

Kohlmann B., Nix H., Shaw D.D. (1988). Environmental predictions and distributional limits of chromosomal taxa in the Australian grasshopper Caledia captiva (F.). Oecologia 75: 483-493.

Kondrashov A.S., Kondrashov F.A. (1999). Interactions among quantitative traits in the course of sympatric speciation. Nature 400: 351-354.

Krusemann G., Jeekel C.A.W. (1967). Stenobothrus (Stenobothrodes) cotticus nov.spec., a new grasshopper from the French Alps (Orthoptera, Acrididae). Entomologische Berichten (Amsterdam) 27(1):1-7.

Kruuk L.E.B. (1997). Barriers to gene flow: a Bombina (fire-bellied toad) hybrid zone and multilocus cline theory. D. Phil. Thesis, University of Edinburgh, Scotland.

Lang G. (1994). Quartäre Vegetationsgeschichte Europas. 462 pp. Gustav Fischer Verlag, Jena. 
Lockey K.M. (1988). Lipits of the insect cuticle: origin, composition and function. Comp. Biochem. Physiol. 81B: 223-227.

Maderbacher M., Bauer C., Herler J., Postl L., Makasa L., Sturmbauer C. (2008). Assessment of traditional versus geometric morphometrics for discriminating populations of the Tropheus moorii species complex (Teleostei: Cichlidae), a Lake Tanganyika model for allopatric speciation. J Zool Syst Evol Res. 46:153-161.

Mallet J. (1995). A species definition for the Modern Synthesis. Trends in Ecology and Evolution 10: 294-299.

Mallet J. (2005). Hybridization as an invasion of the genome. Trends Ecol Evol 20 (5): 229-237.

Mahalanobis P.C. (1936). On the generalized distance in statistics. Proceedings of the National Institute of Sciences of India 12:49-55.

MacCallum CJ, Nürnberger B, Barton NH, Szymura JM. (1998). Habitat preference in the Bombina hybrid zone in Croatia. Evolution 52(1): 227-239.

Maynard-Smith J., Smith N.H., O'Ronrke M., Spratt B.G. (1993). How clonal are bacteria? Proc. Natl. Acad. Sci. USA 90:4384-4388.

Mayr E. (1942). Systematics and the origin of species. Columbia University Press, New York.

Mayr E. (1962). Accident or design: The paradox of evolution. Seiten 1-14 in: The Evolution of Living Organisms (G. W. Leeper, Ed.). Melbourne University Press, Melbourne, Australia.

Mayr E. (1963). Animal Species and Evolution. Harvard University Press, Cambridge/Mass.

Mayr E. (1967): Artbegriff und Evolution. Paul Parey, Hamburg und Berlin. 
McCune A.R., Lovejoy R. (1998). The relative rate of sympatric and allopatric speciation in fishes 17-185 in Howard D.J., Berlocher S.H. eds. Endless forms: species and speciation. Oxford Univ. Press, New York.

Meyer A. (1989). Costs and benefits of morphological specialization: feeding performance in the trophically polymorphic Neotropical cichlid fish, Cichlasoma citrinellum. Oecologia 80: 431-436.

Mitchell A. (2005) GIS Analysis Volume 2: Spatial Measurements und Statistics. ESRI Press.

Morrison D.F. (1967). Multivariate Statistical Methods. McGraw-Hill Book Company, New York.

Neems R.M., Butlin R.K. (1994). Variation in cuticular hydrocarbons across a hybrid zone in the grasshopper Chorthippus parallelus. Proc. R. Soc. Lond. 257B: 135-140.

Nielsen O.F. (2003). Chorthippus jutlandica sp. nov. a new grasshopper found in Jutland, Denmark (Saltatoria, Acrididae, Gomphocerinae). Entomogiske Meddelelser 71, 4152.

Nolte, A.W., Sheets, H.D. (2005). Shape based assignments tests suggest transgressive phenotypes in natural sculpin hybrids (Teleostei, Scorpaeniformes, Cottidae). Frontiers in Zoology 2:11.

Noor M. (1999). Reinforcement and other consequences of sympatry. Heredity 83: 503508.

Otte D., Endler J.A. (1989). Speciation and Its Consequences. Sinauer Associates, Sunderland MA. 
Penin X., Berge C., Baylac, M. (2002). Ontogenetic study of the skull in modern humans and the common chimpanzee: neotenic hypothesis reconsidered with a tridimensional procrustes analysis. Am. J. Phys. Anthrop. 118: 50-62.

Perdeck A.C. (1957). The isolating value of specific song patterns in two sibling species of grasshoppers (Chorthippus brunneus Thunb. and C. biguttulus L.). E.J. Brill, Leiden.

Pretorius E. (2005). Using geometric morphometrics to investigate wing dimorphism in males and females of Hymenoptera - a case study based on the genus Tachysphex Kohl (Hymenoptera: Sphecidae: Larrinae). Australian Journal of Entomology 44: 113-121.

Quenouille M. H. (1956). Notes on bias in estimation. Biometrika 43: $353 \mathrm{ff}$.

Ragge D. (1981). An unusual song pattern in the Chorthippus mollis group (Orthoptera: Acrididae): local variant or hybrid population? J. Nat. Hist. 15:995-1002.

Ragge D. (1984). The Le Broc grasshopper population: further evidence of its hybrid status (Orthoptera: Acrididae). J. Nat .Hist. 18:921-925.

Ragge D.R., Reynolds W.J. (1998). The songs of the grasshoppers and crickets of Western Europe. Nat Hist Mus London.

Rasband W.S. (1997-2005). ImageJ, US National Institutes of Health, Bethesda MD.

Ritchie M.G., Phillips S.D.F. (1998). The genetics of sexual isolation. In: Howard D.J., Berlocher H. (eds) Endless forms - species and speciation. Oxford University Press, New York, 291-308.

Rohlf F.J., Slice, D.E. (1990). Extensions of the Procrustes method for the optimal superimposition of landmarks. Systematic Zoology 39: 40-59.

Rohlf F.J. (2004). tpsDig, digitize landmarks and outlines, version 2.0. Department of Ecology and Evolution, State University of New York at Stony Brook. 
Ross C.L., Harrison R.G. (2002). A fine-scale spatial analysis of the mosaic hybrid zone between Gryllus firmus and Gryllus pennsylvanicus. Evolution 56: 2296-2312.

Rüber L., Verheyen E., Sturmbauer C., Meyer A. (1998). Lake level fluctuations and speciation in rock-dwelling cichlid fish in Lake Tanganyika, East Africa 225-240. In: Evolution on Islands, P.R. Grant. ed., Oxford University Press.

Saccheri I., Kuussaari M., Kankare M., Vikman P., Fortelius W., Hanski I. (1998). Inbreeding and extinction in a butterfly metapopulation. Nature 392: 491-494.

Saldamando C.I., Tatsuta H., Butlin R.K. (2005). Hybrids between Chorthippus brunneus and C-jacobsi (Orthoptera: Acrididae) do not show endogenous postzygotic isolation. Biol J Linn Soc 84:195-203.

Schluter D. (1998). Ecological causes of speciationIn: Howard D. J. Berlocher S. H. Endless forms: species and speciation. Oxford Univ. Press, New York. 114-129.

Schluter D. (2001). Ecology and the origin of species. Trends in Ecology and Evolution, 16: $372-380$.

Schönemann P.H., Carroll R.M. (1970). Fitting one matrix to another under choice of a central dilation and a rigid motion. Psychometrika, 35: 245-255.

Schütze H., Elsner N. (2001). Stridulatory pattern generation in acridid grasshoppers: metathoracic interneurons in Stenobothrus rubicundus (Germar 1817). J Comp Physiol A 187: 529-540.

Schwenk K., Brede N., Streit B. (2008). Extent, processes and evolutionary impact of interspecific hybridization in animals. Philos. Trans. R. Soc. Lond. B 363: 2805-2811.

Searcy W.A., Andersson M. (1986). Sexual selection and the evolution of song. Annu. Rev. Syst. 17:507-533. 
Searle S.R. (1971). Linear Models, New York: John Wiley. (1979), "Notes on Variance Component Estimation: A De-tailed Account of Maximum Likelihood and Kindred Method-ology," Paper No. BU-673-M, Biometrics Unit, Cornell Univer-sity.

Seehausen O. (2004). Hybridization and adaptive radiation. Trends Ecol Evol 19 (4):198207.

Seehausen O. (2006). African cichlid fish: a model system in adaptive radiation research. Proc R Soc Lond Ser B. 273:1987-1998.

Shaw K.L. (1998). Species and the diversity of natural groups. In: Endless Forms: Species and Speciation (Howard D.J., Berlocher S.J.). 44-56. Oxford University Press, Oxford.

Sheets H.D. (2000). Integrated morphometric package (IMP) [online]. Available from http://www2. canisius.edu/,sheets/ [accessed 22 July 2009].

Sradnick J. (2006). Biogeographische und ethologische Untersuchungen an den Feldheuschrecken Stenobothrus clavatus und Stenobothrus rubicundus und deren Hybriden (Diplomarbeit; Universität Göttingen).

Stumpner A., Helversen O.v., (1994) Song production and song recognition in a group of sibling grasshopper species (Chorthippus dorsatus, Ch. dichrous and Ch. loratus: Orthoptera, Acrididae). Bioacoustics 6:1-23.

Swenson N.G. (2008). The past and future influence of geographic information systems on hybrid zone, phylogeographic and speciation research. Journal of Evolutionary Biology 21: 421-434.

Tomlinson R.F. (1984). Geographic information systems: the new frontier. Oper. Geogr. 5: $31-35$. 
Tregenza T., Pritchard V.L., Butlin R.K. (2000a). The origin of premating reproduktive isolation: testing hypotheses in the grasshopper Ch. parallelus. Evolution 54: 16871698.

Tregenza T., Pritchard V.L., Butlin R.K. (2000b). Patterns of divergence between populations of the meadow grasshopper Ch. parallelus. Evolution 54: 574-584.

Tzedakis P.C. (1993). Long-term tree populations in northwest Greece through multiple Quaternary climatic cycles. Nature 364, 437-440.

Tzedakis P.C., Lawson I.T., Frogley M.R., Hewitt G.M., Preece, R.C. (2002). Buffered vegetation changes in a Quaternary refugium: evolutionary implications. Science 297, 2044-2047.

Vedenina V.Y. Helversen O v. (2003). Complex courtship in a bimodal grasshopper hybrid zone. Behav. Ecol. Sociobiol. 54: 44-54.

Vedenina V.Y. (2005). Acoustic communication and sexual selection in Orthoptera (Insecta) Zhurnal Obshchei Biologii 66 : 336-345.

Vedenina V.Y., Panyutin A.K., Helversen O.v. (2007). The unusual inheritance pattern of the courtship songs in closely related grasshopper species of the Chorthippus albomarginatus-group (Orthoptera: Gomphocerinae). J Evol Biol 20: 260-277.

Vedenina V.Y., (2010). Variation in complex courtship traits across a hybrid zone between the grasshopper species of the Chorthippus albomarginatus group. Biological Journal of the Linnean Society. In press.

Via S. (2001). Sympatric speciation in animals: the ugly duckling grows up. Trends in Ecology and Evolution 16: 381-390.

Viloria A.L., Pyrcz T.W., Wojtusiak J., Ferrer-Paris J.R., Beccaloni G.W., Sattler K., Lees D.C. (2003). A brachypterous butterfly? Proc. R. Soc. Lond. B 270: 21-S24. 
Virdee S.R., Hewitt G.M. (1992). Postzygotic isolation and Haldane's rule in a grasshopper. Heredity 69: 527-538.

Virdee S.R., Hewitt G.M. (1994). Clines for hybrid dysfunction in a grasshopper hybrid zone. Evolution 48: 392-407.

Wall R., Begon M. (1987). Population density, phenotype and reproductive output in the grasshopper Chorthippus brunneus. Ecol. Entomol. 12: 331-339.

Waltari E., Hijmans R.J., Peterson A.T., Nyari A.S., Perkins S.L., Guralnick R.P., (2007). Locating pleistocene refugia: comparing phylogeographic and ecological niche model predictions. PLoS ONE 2 (7): 563ff.

Ware C., Knight W., Wells D. (1991). Memory intensive algorithms for multibeam bathymetric data: Computers und Geosciences v. 17, 7: 985-993.

Weaver K.N., Alfano S.E., Kronquist A.R., und Reeder D.M. (2009). Healing rates of wing punch wounds in freeranging little brown myotis (Myotis lucifugus). Acta Chiropterologica 11: 220-223.

West-Eberhard M.J. (1983). Sexual selection, social competition, and speciation. Q. Rev. Biol. 58: 155-183.

Wiley E.O. (1978). The evolutionary species concept reconsidered. Systematic Zoology, 27: 17-26.

Willemse F. (1979). Stenobothrus (Stenobothrodes) clavatus spec. nov. from Greece (Orthoptera, Acrididae). Entomol. Ber. 39: 154-157.

Willemse F. (1985). A key to the Orthoptera species of Greece. - Fauna Graeciae II. Helenic Zoological Society, Athens, $288 \mathrm{p}$.

Willmann R. (1985). Die Art in Raum und Zeit. Das Artkonzept in der Biologie und Paleaontologie. Parey Verlag, Hamburg, Berlin. 
Willott S.J., Hassall M. (1998). Life-history responses of British grasshoppers (Orthoptera: Acrididae) to temperature change. Funct. Ecol. 12: 232-241.

Yanchukov A.W., Hofman S., Szymura J.M., (2006). Hybridization of Bombina bombina and B. variegata (Anura, Discoglossidae) at a sharp ecotone in western Ukraine: comparisons across transects and over time. Evolution 60: 583-600.

Zahavi A. (1987). The theory of signal selection and some of its implications. In: Delfino VP (ed) Proceedings of international symposium on biology and evolution. Adriatrica Editrica, Bari, 305-325

Zrzavý J., Storch D., Mihulka S. (2009). Evolution -ein Lese-Lehrbuch-. Spektrum Akademischer Verlag Heidelberg 


\section{Anhang}

\subsection{Abkürzungsverzeichnis}

\begin{tabular}{|c|c|}
\hline$\circ$ & Grad \\
\hline$\%$ & Prozent \\
\hline Abb. & Abbildung \\
\hline B. & Bombina \\
\hline bzw. & beziehungsweise \\
\hline bzgl. & bezüglich \\
\hline${ }^{\circ} \mathrm{C}$ & Grad Celsius \\
\hline ca. & circa \\
\hline Ch. & Chorthippus \\
\hline $\mathrm{cm}$ & Centimeter \\
\hline dpi. & dots per inch: eine Maßeinheit für die Auflösung eines Fotos \\
\hline et al. & und weitere (lat.: et alii) \\
\hline GPS & Global Positioning System \\
\hline $\mathrm{h}$ & Stunde (n) \\
\hline Inc. & Incorporated \\
\hline $\mathrm{kHz}$ & Kilohertz \\
\hline $\mathrm{km}$ & Kilometer \\
\hline $\mathrm{km}^{2}$ & Quadratkilometer \\
\hline lat. & Latein \\
\hline LHB & linkes Hinterbein \\
\hline LM & Landmarke \\
\hline $\min$ & Minute (n) \\
\hline $\mathrm{m}$ & Meter \\
\hline $\mathrm{mm}$ & Millimeter \\
\hline $\mathrm{ms}$ & Millisekunden \\
\hline $\mathrm{Nr}$ & Nummer \\
\hline pers. Mitt. & persöhnliche Mitteilung \\
\hline pp. engl. & pages (Seiten) \\
\hline $\mathrm{HB}$ & rechts Hinterbein \\
\hline
\end{tabular}


S

Sekunde

S.o.

siehe oben

sp

Species

St.

Stenobothrus

s.u. siehe unten

sym. synonym

Tab. Tabelle

TPS Thin Plate Spline

u.a. unter anderem

u. a. m. und anderes mehr

unveröff. Unveröffentlicht

u. U. unter Umständen

v. a. vor allem

vgl. vergleiche

z.B. zum Beispiel

z.T.

zum Teil

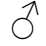

Männchen

+

Weibchen 


\section{E B E N S L A U F}

\section{Persönliche Daten:}

Vor- und Zuname:

Jan Sradnick

Geburtstag:

24.11.1981

Geburtsort:

Cottbus

Wohnort:

37075 Göttingen

Staatsangehörigkeit:

deutsch

Familienstand:

verheiratet

\section{Wissenschaftliche Tätigkeit:}

10/2007- heute

11/2006-10/2007
Wissenschaftliche Hilfskraft am Johann-FriedrichBlumenbach-Institut für Zoologie und Anthropologie an der Universität Göttingen; Abteilung Neurobiologie

Studentische Hilfskraft am Johann-FriedrichBlumenbach-Institut für Zoologie und Anthropologie an der Universität Göttingen; Abteilung Neurobiologie

Wissenschaftlicher Werdegang:

10/2007- heute

$10 / 2001-10 / 2007$
Promotionsstudium der Biologie an der Universität Göttingen

Studium der Biologie an der Universität Göttingen Abschluss: Diplom (Univ.)

\section{Schulbildung:}

$09 / 1995-05 / 2001$

$09 / 1988-07 / 1995$
Gymnasium in Beeskow

Abschluss: Allgemeine Hochschulreife

Grundschule in Friedland (Niederlausitz) 


\section{besuchte Fachtagungen und Seminare:}

$05 / 2006$

23. -25.02 .2007$

07.05.2007

14.05.2007

21.01.2008

07. - 09.04.2008

06. - 08.06.2008

12. -13.09 .2008$

19. -22.09 .2008$

05. -08.03 .2009$

13.05.2009

19. -20.09 .2009$

21. -25.06 .2009$

25. -28.09 .2009$
Symposium in Bursfelde, internationales

Forschungsgruppentreffen

Graduiertentreffen der Deutschen Zoologischen Gesellschaft, Bayreuth (Poster)

Internationales Symposium der Akademie der Wissenschaften Göttingen (Vortrag)

Zoologisches Kolloquium, HU- Berlin, (Vortrag)

Zoologisches Kolloquium, Göttingen, (Vortrag)

Systematics 2008, Tagung. Göttingen, (Poster)

Evolutionsökologische Seminar der Universität Nürnberg-Erlangen (Vortrag)

Symposium in Eygelshoven, internationales Forschungsgruppentreffen, Niederlande (Vortrag)

Jahresversammlung der Deutschen Zoologischen Gesellschaft, Jena, (Poster)

Graduiertentreffen der Deutschen Zoologischen Gesellschaft, München, (Evolution), (Poster)

Arbeitsgruppenseminar (Bielefeld), (Vortrag)

Symposium in Eygelshoven, internationales Forschungsgruppentreffen, Holland (Vortrag)

Internationaler Kongress der Orthopterology, Antalya, (Poster, Vortrag)

Jahresversammlung der Deutschen Zoologischen Gesellschaft, (Regensburg) (Poster)

\section{Auslandsaufenthalte:}

$07-08 / 2005$

$06-09 / 2006$

$05-08 / 2007$

$07-08 / 2008$

$07 / 2009$
Ioannina, Griechenland

Ioannina, Griechenland

Ioannina, Griechenland

Ioannina, Griechenland

Ioannina, Griechenland 


\section{Veröffentlichungen:}

Publikationen:

Ostrowski TD, Sradnick J, Stumpner A, Elsner N. The Elaborate courtship behavior of Stenobothrus clavatus Willemse, 1979 (Acrididae: Gomphocerinae) Journal of Orthoptera Research 18(2):171-182. 2009.

Elsner N, Klöpfel A, Sradnick J. Das geschmähte fremde Blut - Bastardierung als Motor der Evolution . Evolution - Zufall und Zwangsläufigkeit der Schöpfung, Wallenstein Verlag 2009. 


\section{Danksagung}

Bei Prof. Dr. Norbert Elsner möchte ich mich herzlich für die Betreuung und für die Bereitstellung des sehr interessanten Themas bedanken. Sowie für die gemeinsame Zeit in Griechenland und Göttingen.

Herrn Prof. Dr. A. Stumpner danke ich für gute Anregungen und die Übernahme des Korreferats.

Des Weiteren danke ich Sylvia Fähsing, Alexander Hübner und Anna Schüth für die Hilfe bei der Arbeit in Griechenland sowie für die schöne Zeit in Göttingen. Vor allem möchte ich mich bei Tim Ostrowski für die Hilfe bei den Vorarbeiten dieser Arbeit bedanken

Ph.D. Dr. Varvara Vedenina und Dr. Fer Willemse möchte ich für die Anregungen und vielen hilfreichen Gespräche danken.

Für die tatkräftige Unterschützung bei allen großen und kleinen technischen Problemen möchte ich mich bei allen Mitarbeitern der Werkstätten der Zoologie bedanken.

Ebenfalls für die nette Atmosphäre und viele nette, hilfreiche Gespräche möchte ich mich bei Matthias Schink, Magret Winkler, Gudrun Matthes und Patricia Sprysch bedanken.

Danke an alle weiteren Mitarbeiter der Abteilungen für Neurobiologie und Zellbiologie für die freundliche Atmosphäre und die Unterstützung in Alltag.

Ganz herzlichen Dank an meine Schwiegereltern, die bis zur letzten Minute diese Arbeit Korrektur gelesen haben.

Abschließend danke ich meinen Eltern, Geschwistern und Großeltern, die mich fortwährend unterstützt haben. Ich konnte mich immer auf sie verlassen.

Und schließlich danke ich meiner Frau Anja dafür dass die immer an meiner Seite steht und mir so viel Wärme gibt. 